\title{
Muon Neutrino to Electron Neutrino Oscillation in $\mathrm{NO} \nu \mathrm{A}$
}

\author{
A THESIS \\ SUBMITTED TO THE FACULTY OF THE GRADUATE SCHOOL \\ OF THE UNIVERSITY OF MINNESOTA \\ BY
}

Kanika Sachdev

IN PARTIAL FULFILLMENT OF THE REQUIREMENTS FOR THE DEGREE OF Doctor of Philosophy

Prof. Daniel Cronin-Hennessy \& Prof. Gregory Pawloski

August, 2015 
(C) Kanika Sachdev 2015

ALL RIGHTS RESERVED 


\section{Acknowledgements}

I want to begin with thanking my undergraduate teacher, Adarsh Shroff- I would not be a physicist had it not been for your encouragement.

Thank you, to my advisers, Daniel Cronin-Hennessy and Gregory Pawloski, for your guidance, support, and patience, and for sharing your intuition in Physics with me.

Thank you, Mayly Sanchez and Patricia Vahle, for your trust and encouragement.

Thank you, NO $\nu$ A graduate students at Minnesota- Dominick, Jan, Minerba, Nick and Susan, for helping me learn the ropes early on and teaching me how to stand on my own two feet.

Thank you, Gavin Davies, Chris Backhouse and Evan Niner, with whom I shared this nerve-wracking, life-changing and thrilling experience of bringing a new experiment online and extracting the first physics results from it. I wouldn't choose different friends if I had to do it all over again.

Thank you, mom, dad, Maggi and Larry, for everything; this journey would be unimaginable without you. 


\section{Dedication}

To my parents, Ravi and Archana, my sister, Maggi, and Larry. 


\begin{abstract}
NOvA is a long-baseline neutrino oscillation experiment optimized for electron neutrino $\left(\nu_{e}\right)$ appearance in the NuMI beam, a muon neutrino $\left(\nu_{\mu}\right)$ source at Fermilab. It consists of two functionally identical, nearly fully-active liquid-scintillator tracking calorimeters. The near detector (ND) at Fermilab is used to study the neutrino beam spectrum and composition before oscillation, and measure background rate to the $\nu_{e}$ appearance search. The far detector, $810 \mathrm{~km}$ away in Northern Minnesota, observes the oscillated beam and is used to extract oscillation parameters from the data. $\mathrm{NO} \nu \mathrm{A}$ 's long baseline, combined with the ability of the NuMI beam to operate in the antineutrino mode, makes $\mathrm{NO} \nu \mathrm{A}$ sensitive to the last unmeasured parameters in neutrino oscillations- mass hierarchy, $\mathrm{CP}$ violation and the octant of mixing angle $\theta_{23}$. This thesis presents the search for $\nu_{e}$ appearance in the first data collected by the $\mathrm{NO} \nu \mathrm{A}$ detectors from October 2013 till May 2015.

Studies of the NuMI neutrino data collected in the $\mathrm{NO} \nu \mathrm{A}$ near detector are also presented, which show large discrepancies between the ND simulation and data. Muonremoved electron (MRE) events, constructed by replacing the muon in $\nu_{\mu}$ charged current interactions by a simulated electron, are used to correct the far detector $\nu_{e}$ appearance prediction for these discrepancies.

In the analysis of the first data, a total of $6 \nu_{e}$ candidate events are observed in the far detector on a background of 1 , a $3.46 \sigma$ excess, which is interpreted as strong evidence for $\nu_{e}$ appearance. The results are consistent with our expectation, based on constraints from other neutrino oscillation experiments.

The result presented here differs from the officially published $\nu_{e}$ appearance result from the $\mathrm{NO} \nu \mathrm{A}$ experiment where the systematic error is assumed to cover the MRE correction.
\end{abstract}




\section{Contents}

Acknowledgements $\quad$ i

Dedication $\quad$ ii

Abstract $\quad$ iii

List of Tables $\quad$ x

List of Figures $\quad$ xii

1 Neutrino Physics 1

1.1 Neutrinos in the Standard Model . . . . . . . . . . . . . . . . . . . 1

1.1.1 Weak Interaction . . . . . . . . . . . . . . . . . . 2

1.1.2 Free Neutrinos . . . . . . . . . . . . . . . 3

1.2 Extending the Standard Model . . . . . . . . . . . . . . . . . . 4

1.2.1 Majorana Mass of Neutrinos . . . . . . . . . . . . . . 4

1.3 Neutrino Oscillations . . . . . . . . . . . . . . . 6

1.3.1 Oscillations in Vacuum . . . . . . . . . . . 6

1.3.2 Oscillations in Matter . . . . . . . . . . . . . . 8

2 Status of Neutrino Oscillation Measurements 12

2.1 Conventions and Nomenclature . . . . . . . . . . . . . . . . . 12 
2.2 Oscillation Parameter Measurements . . . . . . . . . . . . . . . . . 13

2.2.1 $\theta_{12}$ and $\Delta m_{21}^{2}$ Measurements $\ldots \ldots \ldots \ldots \ldots$

2.2.2 $\theta_{23}$ and $\left|\Delta m_{32}^{2}\right|$ Measurements . . . . . . . . . . 16

$2.2 .3 \quad \theta_{13}$ Measurements . . . . . . . . . . . . . . . 17

2.3 Unknown Parameters . . . . . . . . . . . . . . . . . . . 18

2.3 .1 Mass Hierarchy . . . . . . . . . . . . . . . 18

2.3 .2 CP Violation . . . . . . . . . . . . . . . . 20

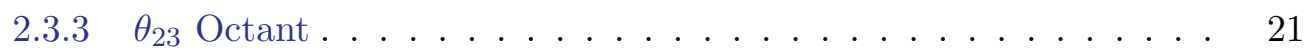

$2.4 \nu_{\mu} \rightarrow \nu_{e}$ on Long Baseline . . . . . . . . . . . . . 21

2.4 .1 Experimental Observation . . . . . . . . . . . . . . . 24

3 The $\mathrm{NO} \nu \mathrm{A}$ Experiment $\quad 25$

3.1 The NuMi Beam . . . . . . . . . . . . . . . . . . . . 27

3.1 .1 The Accelerator Complex . . . . . . . . . . . . . . . 30

3.1 .2 Slip-Stacking . . . . . . . . . . . . . . . . . 31

3.1.3 The NuMi Beamline . . . . . . . . . . . . . . . . . . . 31

3.2 The NO $\nu$ A Detector Design . . . . . . . . . . . . . . . . 32

3.2 .1 The $\mathrm{NO} \nu \mathrm{A}$ Unit Cell . . . . . . . . . . . . . . . . . . . 32

3.2 .2 Liquid Scintillator . . . . . . . . . . . . . 33

3.2 .3 Optical Fiber . . . . . . . . . . . . . . . 35

3.2.4 Avalanche Photodiode (APD) . . . . . . . . . . . 36

3.2 .5 PVC Modules . . . . . . . . . . . . . . . . . . 37

3.2 .6 Detector Assembly . . . . . . . . . . . . . . . . . 40

4 Data Acquisition and Triggering Systems $\quad 43$

4.1 Overview of $\mathrm{NO} \nu \mathrm{A}$ DAQ System . . . . . . . . . . . . . . 43

4.2 Clock and Triggering . . . . . . . . . . . . . . . . . 44

4.3 Readout ........................... 46

4.3.1 APD Sag and Ringing . . . . . . . . . . . . 47 
4.4 Timing Peak . . . . . . . . . . . . . . . . . . . . 48

4.4 .1 Far Detector . . . . . . . . . . . . . . . . . 48

4.4.2 Near Detector . . . . . . . . . . . . . . . . . . 50

$5 \nu_{e}$ Appearance Analysis Overview $5 \mathbf{5 2}$

5.1 Oscillation Analysis Steps . . . . . . . . . . . . . . . . . 52

5.1 .1 Blind Analysis . . . . . . . . . . . . . . . 52

5.1 .2 Cosmic Background Estimate . . . . . . . . . . 53

5.1 .3 Beam Background Estimate . . . . . . . . . . . . . . 54

5.1 .4 Extrapolation . . . . . . . . . . . . . . . . 55

5.1 .5 Final Fit . . . . . . . . . . . . . . 55

$5.2 \mathrm{NO} \nu \mathrm{A}$ 's First Data . . . . . . . . . . . . . . . . . 55

5.2.1 Treatment of $64 \mu$ s Delayed Peak in Pre-Shutdown Data . . . . . 56

5.3 Simple Oscillations . . . . . . . . . . . . . . . . . . . 57

$\begin{array}{lll}6 & \text { Simulation } & 59\end{array}$

6.1 Particle Simulation . . . . . . . . . . . . . . . . . . 59

6.1 .1 Beam .............................. 59

6.1 .2 Neutrinos . . . . . . . . . . . . . . . . . 60

6.1.3 Particles in Detector . . . . . . . . . . . . . . . 61

6.2 Detector Response Simulation . . . . . . . . . . . . . . . 62

6.2 .1 Photon Transport . . . . . . . . . . . . . . 62

6.2 .2 Readout Simulation . . . . . . . . . . . . . . 63

6.3 Tuning to Data . . . . . . . . . . . . . . . 64

6.3 .1 APD Sag Simulation . . . . . . . . . . . . . . 65

6.3 .2 Scintillator Quenching . . . . . . . . . . . . 65

6.3.3 Diblock and Bad Channel Masking . . . . . . . . . . . . 68 
7 Event and Energy Reconstruction $\quad 70$

7.1 Calibration .............................. 70

7.1.1 Attenuation Correction . . . . . . . . . . . . . . 71

7.1 .2 Absolute Energy Scale . . . . . . . . . . . . . . . . . 73

7.1 .3 Timing resolution . . . . . . . . . . . . . . . 74

7.2 Reconstruction Steps . . . . . . . . . . . . . . . . . . 75

7.3 Slicing Algorithm . . . . . . . . . . . . . . . . . 77

$7.4 \nu_{e}$ Reconstruction . . . . . . . . . . . . . . . . . 80

7.4 .1 Hough Transform . . . . . . . . . . . . . . . . . 80

7.4 .2 Elastic Arms Vertex . . . . . . . . . . . . . . . . 82

7.4.3 Fuzzy-k Prong Reconstruction . . . . . . . . . . . . . . . . 83

7.4.4 $\nu_{e}$ Event Energy Reconstruction . . . . . . . . . . . . . . 85

$7.5 \nu_{\mu}$ Reconstruction Chain . . . . . . . . . . . . 86

$8 \nu_{e}$ Event Selection $\quad 89$

$8.1 \nu_{e}$ Selection Cuts . . . . . . . . . . . . . . . . . 89

8.1 .1 Data Quality Cuts . . . . . . . . . . . . . . . . 89

8.1.2 Preselection and Cosmic Rejection Cuts . . . . . . . . . . . 92

8.1.3 Performance of Preselection . . . . . . . . . . . . . . 98

$8.2 \nu_{e}$ Particle Identification $\ldots \ldots \ldots \ldots$

8.2.1 Likelihood-based Particle Identification . . . . . . . . . . . . . . 101

8.3 Final Cosmic Background Prediction . . . . . . . . . . . . . . . . . 108

9 Near Detector Data 112

9.1 Near Detector Data-MC Comparisons . . . . . . . . . . . . . . . . . 113

9.1.1 ND $\nu_{e}$ Event Selection . . . . . . . . . . . . . . . . 113

9.1 .2 ND $\nu_{\mu}$ Event Selection . . . . . . . . . . . . . . . . . . 119

9.2 Interpretation . . . . . . . . . . . . . . . . . . . . 122

9.2.1 Fitting For Interaction Type . . . . . . . . . . . . . . 123 
9.2.2 Fitting For Interaction Mode . . . . . . . . . . . . . . . 125

10 Muon Removed Electron Events 134

10.1 MRE Event Generation . . . . . . . . . . . . . . . . . . . . . 134

10.1 .1 Muon Removal . . . . . . . . . . . . . . . . . . . . . 135

10.1.2 Muon and Hadron Energy Disentaglement . . . . . . . . . . 136

10.1.3 Performance of Muon-Removal . . . . . . . . . . . . . . . 139

10.1.4 Insertion of Simulated Electron . . . . . . . . . . . . . 141

10.2 MRE ND Data-MC Comparison . . . . . . . . . . . . . . . 141

10.2.1 MRE Event Selection . . . . . . . . . . . . . . . . . . . 141

10.2.2 MRE with Interaction Mode Scaling . . . . . . . . . . . 145

10.3 MRE Correction On Signal Prediction . . . . . . . . . . . . . . . 147

10.3.1 Efficiency by Shower and Hadronic Energy . . . . . . . . . . 147

11 Decomposition and Extrapolation $\quad 155$

11.1 Decomposition . . . . . . . . . . . . . . 156

11.1.1 Proportional Decomposition . . . . . . . . . . . . 156

11.1.2 Decomposition, Upon Scaling by Interaction Type . . . . . . . 156

11.1.3 Decomposition, Upon Scaling by Interaction Mode . . . . . . . . 156

11.1.4 Summary of Decomposition . . . . . . . . . . . . . 157

11.2 Background Extrapolation . . . . . . . . . . . 157

11.3 Signal Extrapolation . . . . . . . . . . . . . . . . 160

11.4 Results of Extrapolation . . . . . . . . . . . . . . . . . 163

12 Error Estimates $\quad 167$

12.1 Calibration Error . . . . . . . . . . . . . . . . . 167

12.2 Interaction Model Uncertainties . . . . . . . . . . . . . 169

12.2.1 Beam Flux Errors . . . . . . . . . . . . . . 170

12.2.2 Neutrino Interaction Model Uncertainty . . . . . . . . . . . . 171 
12.3 Birks Suppresion . . . . . . . . . . . . . . . . . . 172

12.4 Decomposition Uncertainty . . . . . . . . . . . . . . . . . . . 172

12.5 Uncertainty on MRE Correction . . . . . . . . . . . . . . . 173

12.6 Other Smaller Effects _. . . . . . . . . . . . . 176

12.6.1 Containment and Rock Contamination . . . . . . . . . . . . 176

12.6.2 Simulation Statistics and Normalization . . . . . . . . . 177

12.6 .3 Alignment . . . . . . . . . . . . . . . . . . 177

12.6.4 Light Level . . . . . . . . . . . . . . . . . . . . . 178

12.7 Summary . . . . . . . . . . . . . . . . . . 178

13 Results of $\nu_{e}$ Appearance Analysis $\quad \mathbf{1 8 0}$

13.1 PID Sideband . . . . . . . . . . . . . . . . . . . 181

$13.2 \nu_{e}$ Appearance Result . . . . . . . . . . . . . . . . . 181

13.3 Analysis of the Result . . . . . . . . . . . . . . . 187

13.3.1 Significance of Observation: Simple Treatment . . . . . . . . . 191

13.3.2 Fit to Oscillation Parameters . . . . . . . . . . . . . . 192

13.3.3 Feldman-Cousins Method for Confidence Interval . . . . . . . . . 195

13.3.4 Result from Alternative PID, LEM . . . . . . . . . . . . . . . 198

13.3.5 Comparison with Official NO $\nu \mathrm{A} \nu_{e}$ Appearance Result . . . . . 198

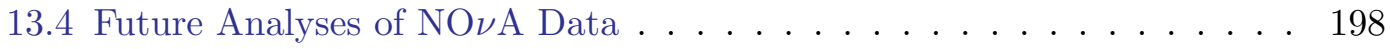

$\begin{array}{ll}\text { References } & 200\end{array}$ 


\section{List of Tables}

5.1 Far detector exposure and livetime . . . . . . . . . . . . . 56

8.1 Containment cuts in ND . . . . . . . . . . . . . . . . . 97

$8.2 \nu_{e}$ selection cut flow in the FD $\ldots \ldots \ldots \ldots$

8.3 Summary of $\nu_{e}$ preselection cuts applied in the far detector . . . . . 100

8.4 Performance of LID . . . . . . . . . . . . . . . . . . . . 110

8.5 Cut flow on cosmic ray background in out-of-time NuMI data . . . . . . 110

9.1 Near detector $\nu_{e}$ selection cuts . . . . . . . . . . . . . . . . 113

$9.2 \nu_{e}$ event selection efficiency in Near Detector . . . . . . . . . . . . . . 114

$9.3 \nu_{\mu}$ CC selection criteria in the ND . . . . . . . . . . . . . 120

9.4 Description of interaction type and mode . . . . . . . . . . . 123

9.5 Result of fit for NC, $\nu_{\mu} \mathrm{CC}$ and beam $\nu_{e} \mathrm{CC}$ scales in ND . . . . . . 124

9.6 Result of fit for QE, resonance and DIS scales in ND . . . . . . . . . . 128

10.1 Cut flow of ND MRE data and MC . . . . . . . . . . . . . . . . . . 142

11.1 Result of decomposition of $\nu_{e}$ selected data in the near detector . . . . . 157

11.2 Extrapolated signal and background predictions for $\nu_{e}$ appearance analysis of $\mathrm{NO} \nu \mathrm{A}$ 's first data . . . . . . . . . . . . . . . . . 164

11.3 Predictions with different oscillation scenarios . . . . . . . . . . . . 164

12.1 Calibration systematic error . . . . . . . . . . . . . 170

12.2 Decomposition Error . . . . . . . . . . . . . . . . . . 173 
12.3 Change in MRE corrected extrapolated $\nu_{e}$ signal production due to different selection cuts on the MRE parent $\nu_{\mu}$ CC interaction . . . . . . . 174

12.4 Change in MRE corrected extrapolated $\nu_{e}$ signal production due to different parent matching efficiency cuts on the MRE slice. . . . . . . . . . 176

12.5 Final systematic error (in percentages) on the combined background and signal in the Far Detector. . . . . . . . . . . . . . . . . 179

13.1 Expected signal and background for $\mathrm{NO} \nu \mathrm{A}$ first data along with the systematic and statistical errors on the predictions. . . . . . . . . 180

13.2 LID sideband event counts expectation in the far detector . . . . . . . . 181

13.3 Significance of observation . . . . . . . . . . . . . . . . . . 191 


\section{List of Figures}

1.1 Masses of fundamental particles in the Standard Model . . . . . . . . . 4

1.2 Allowed coherent scattering modes for neutrinos passing through the earth matter . . . . . . . . . . . . . . . . . 9

2.1 Predicted solar neutrino spectrum $[1] \ldots \ldots \ldots$

2.2 Mass hierarchy in neutrinos . . . . . . . . . . . . . . . . 19

2.3 Values of effective Majoarana mass as a function of the lightest neutrino

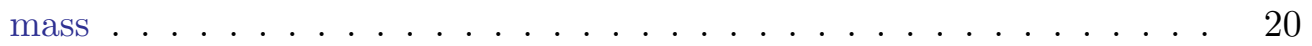

$3.1 \nu_{\mu}$ to $\nu_{e}$ oscillation probability for the NuMI beam at the location of the $\mathrm{NO} \nu \mathrm{A}$ far detector under different hierarchy hypotheses. . . . . . . . . 26

$3.2 P\left(\nu_{\mu} \rightarrow \nu_{e}\right)$ versus $P\left(\bar{\nu}_{\mu} \rightarrow \bar{\nu}_{e}\right)$ for $\mathrm{NO} \nu \mathrm{A} \ldots \ldots \ldots \ldots 27$

3.3 The neutrino energy spectra and flux as a function of the pion energy for different off-axis angles, $\theta . \ldots \ldots \ldots \ldots$

3.4 Simulated neutrino energy spectra at the $\mathrm{NO} \nu \mathrm{A}$ far detector baseline of $810 \mathrm{~km}$. The off-axis location of $\mathrm{NO} \nu \mathrm{A}$ suppress the high neutrino energy

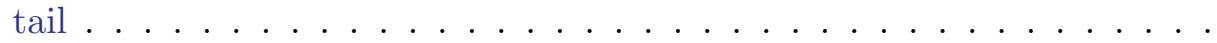

3.5 Schematic of the Fermilab accelerator complex, with upgrades implemented for $\mathrm{NO} \nu \mathrm{A} \ldots \ldots \ldots . \ldots \ldots$

3.6 The NuMI Beamline $[2] \ldots \ldots \ldots \ldots$

3.7 A cell and a cut-out of the $\mathrm{NO} \nu \mathrm{A}$ detectors indicating the orthogonal arrangment of cells in adjacent planes. . . . . . . . . . . . . 
3.8 The emission spectrum of the NO $\nu \mathrm{A}$ scintillator. The figure is from [3]. 34

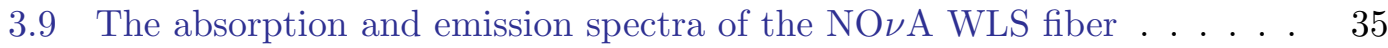

3.10 Schematic of the APD and front-end electronics. . . . . . . . . . . . 37

3.11 Profile of the PVC extrusion showing scalloped corners in the inner and outer walls of the extrusion . . . . . . . . . . . . . . 38

3.12 Schematic of the APD and front-end electronics. . . . . . . . . . . . 39

3.13 Block pivoter during the installation of a block in the far detector hall . 41

4.1 DCM lay out in FD . . . . . . . . . . . . . . . . . . . 44

4.2 Single- and multi-point point readout . . . . . . . . . . . . . . 47

4.3 Readout traces illustrating APD sag. . . . . . . . . . . . . . . . . 48

4.4 Far detector timing peak, over the full $500 \mu$ s window, with all data upto February 16, 2015. . . . . . . . . . . . . . . . . 49

4.5 Near detector timing peak, over the full $500 \mu$ s window, and zoomed in to show the NuMI beam structure. . . . . . . . . . . . . 51

5.1 Timing sideband . . . . . . . . . . . . . . . . 54

5.2 Timing sideband in pre-shutdown data . . . . . . . . . . . . . . 57

6.1 FLUGG's neutrino flux prediction by flavor and sign for the $\mathrm{NO} \nu \mathrm{A}$ detectors in the forward horn current mode . . . . . . . . . . . . 60

6.2 Particle simulation steps in $\mathrm{NO} \nu \mathrm{A} \ldots \ldots . \ldots . \ldots . \ldots . \ldots 62$

6.3 Light collection rate estimate from the ray tracing algorithm . . . . . 63

6.4 Comparison of raw pulse-heights in FD and ND cosmic ray data . . . 65

6.5 $\mathrm{PE} / \mathrm{cm}$ per plane measured in near detector data compared with $\mathrm{MC}$

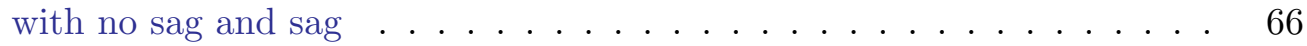

6.6 $d E / d x$ comparison between ND data and MC for candidate proton track 67

6.7 POT exposure in each diblock configuration in data and simulation . . . 69

7.1 Selection of tri-cells associated with a track . . . . . . . . . . . . 71

7.2 Attenuation correction of a far detector cell, and the residual of the fit . 73 
$7.3 d E / d x$ as a function of distance from the muon stopping point as measured in FD cosmic ray data . . . . . . . . . . . . . . . . 74

7.4 Absolute energy calibration in the far detector . . . . . . . . . . . 74

7.5 Timing resolution as a function of $\mathrm{PE}$ in $\mathrm{FD}$ and ND . . . . . . . . 75

7.6 Neutrino interactions of different types in the $\mathrm{NO} \nu \mathrm{A}$ detectors $\ldots . . .76$

7.7 ND and FD data from a single spill reconstructed into separate slices . . 79

7.8 Efficiency and purity of slices in ND simulation . . . . . . . . . . 80

$7.9 \nu_{e}$ reconstruction chain . . . . . . . . . . . . . . . . . 81

7.10 Resolution of reconstructed vertex for $\nu_{e}$ CC events . . . . . . . . . . 82

7.11 Efficiency and purity of reconstructed prongs . . . . . . . . . . . 85

7.12 Reconstructed energy resolution of preselected $\nu_{e}$ charged-current inter-

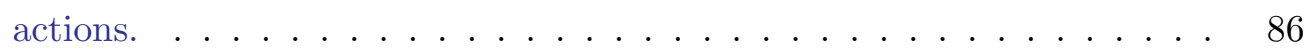

7.13 Muon neutrino energy resolution for quasi-elastic CC interactions. . . . 87

8.1 An FD spill with DCM synchronization problem . . . . . . . . . . . 92

8.2 Flashing of multiple APDs in the FD cosmic data . . . . . . . . . . . . 94

8.3 Neutron interaction depth in the FD . . . . . . . . . . . . . . . . 95

8.4 Minimum distance of events in the far detector from the top and the back faces of the detector . . . . . . . . . . . . . 96

8.5 Transverse momentum fraction of the far detector events with respect to the beam direction . . . . . . . . . . . . . . . . . . 97

8.6 Energy distribution of FD events after preselection . . . . . . . . . 98

8.7 Efficiency of $\nu_{e}$ preselection cuts as a function of number of detector diblocks in the FD, as measured in real conditions MC. . . . . . . . . 99

8.8 Fuzzy-K prongs are reclustered to better fit the electron shower shape. . 102

8.9 Transverse $d E / d x$ per electron energy as a function of distance from the shower core. The distribution is fit with $8.2 \ldots \ldots$. . . . . . . 102

8.10 Muon and electron $d E / d x$ in the second and the tenth plane from the start point . . . . . . . . . . . . . . . . . 
8.11 Longitudinal and transverse likelihood difference for electron, neutral pions and muons . . . . . . . . . . . . . . . . 105

8.12 Distribution of ANN input variables for signal(red) and background (blue)107

8.13 Distribution of LID for different neutrino interactions . . . . . . . . . . 109

8.14 Efficiency of selecting $\nu_{e}$ appearance signal and purity as a function of LID cut . . . . . . . . . . . . . . . . . . . 109

9.1 Near detector fiducial and containment bounds for $\nu_{e}$ selection . . . . . 112

9.2 Data and $\mathrm{MC}$ comparison of $\mathrm{ND} \nu_{e}$ preselected events. . . . . . . . . . 115

9.3 Data and MC comparison of ND $\nu_{e}$ preselected events . . . . . . . 116

9.4 Data and MC comparison of ND $\nu_{e}$ preselected events. . . . . . . . 117

9.5 Data and MC comparison of ND LID selected events. . . . . . . . . . 118

9.6 Data and MC comparison of ND LID selected events . . . . . . . . . . . 119

9.7 Reconstructed quantities for $\nu_{\mu}$ selected events in the ND . . . . . . 121

9.8 Performance of ReMID, the $\nu_{\mu}$ CC PID, on the ND data and MC. . . . 122

$9.9 \chi^{2}$ distributions of fit for $\mathrm{NC}, \nu_{\mu} \mathrm{CC}$ and beam $\nu_{e} \mathrm{CC}$ scales in ND. . . 124

9.10 Result of scaling interaction types in ND MC . . . . . . . . . . . . 125

9.11 Hadronic energy in ND preselected events, split by interaction mode . . 127

9.12 Cross-section as a function of neutrino energy in different modes. For further description of the plot, see $[4] \ldots \ldots$. . . . . . . . . . 129

9.13 Result of scaling interaction modes in ND MC . . . . . . . . . . . 130

9.14 Result of scaling interaction modes in ND MC . . . . . . . . . . . 131

9.15 Result of scaling interaction modes in ND MC . . . . . . . . . . . 132

10.1 MRE event creation process f . . . . . . . . . . . . 136

$10.2 d E / d x$ of muons with and without hadronic contamination. . . . . . . 137

10.3 Variables to measure the performance of muon removal . . . . . . . . 140

10.4 Comparison of MRE events in ND data and MC . . . . . . . . . . 143

10.5 Comparison of MRE events in ND data and MC . . . . . . . . . . . . 144

10.6 MRE in MC with interaction mode scaled by factor from $9.6 \ldots 146$ 
10.7 LID selection efficiency of MRE events in the ND . . . . . . . . . . . . 149

10.8 Efficiency ratio of MRE selection efficiency in data and simulation . . . 150

10.9 Shower multiplicity as a function of primary shower and hadronic energy in MRE events . . . . . . . . . . . . . . . . . . . . . 151

10.10Shower multiplicity as a function of primary shower and hadronic energy in $\mathrm{MRE}$ events . . . . . . . . . . . . . . . . . . . . . 151

10.11A cartoon of hadronic showers of same energy but different multiplicity 152

10.12Impact of MRE correction on FD simulated $\nu_{e}$ signal . . . . . . . . . . . 153

11.1 Reconstructed energy of $\mathrm{NC}$ and $\nu_{\mu} \mathrm{CC}$ backgrounds in the near detector and extrapolated spectra in the far detector. . . . . . . . . . . . 159

11.3 Extraction of a predicted $\nu_{e}$ reconstructed energy spectrum from FD true to reconstructed $\nu_{e}$ energy map and ND true energy spectra for data and

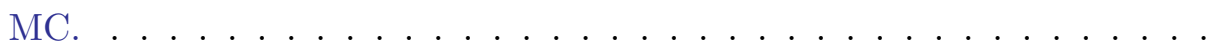

11.2 Reconstructed energy to true energy conversion of the ND data $\nu_{\mu} \mathrm{CC}$

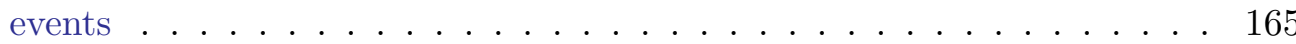

11.4 Reconstructed energy of $\nu_{\mu}$ selected events in the ND, with and without mode scaling. . . . . . . . . . . . . . . . . . . . . . 166

12.1 Evidence of miscalibration in near and far detectors. . . . . . . . . . . . 169

12.2 Beam systematic error on ND Spectra . . . . . . . . . . . . . . . 171

12.3 Change in $\nu_{e}$ selection of MRE events upon changing the $\nu_{\mu}$ selection of the parent interactions. . . . . . . . . . . . . . 175

12.4 Summary of signal and background uncertainties . . . . . . . . . . . 178

$13.1 \nu_{e}$ Appearance candidates in FD NuMI beam data. The gray events are are out-of-time cosmics. . . . . . . . . . . . . . . . . 183

13.2 Timing distribution of FD signal events. The blue line indicate the NuMI beam window boundaries. The two out of time events are cosmic background. ............................ 183 
$13.3 \nu_{e}$ Appearance candidates in FD NuMI beam data. The gray events are are out-of-time cosmics. . . . . . . . . . . . . . . . 184

$13.4 \nu_{e}$ Appearance candidates in FD NuMI beam data. The gray events are are out-of-time cosmics. . . . . . . . . . . . . . . .

$13.5 \nu_{e}$ Appearance candidates in FD NuMI beam data. The gray events are are out-of-time cosmics. . . . . . . . . . . . . . 186

13.6 Distributions of signal events in the NuMI beam window in the FD data. 187

13.7 Vertex distributions of candidate events . . . . . . . . . . . . . . . 188

$13.8 d E / d x$ by plane number from the shower start in the longitudinal and transverse directions for leading showers in the $\nu_{e}$ candidate events $\ldots 189$

$13.9 d E / d x$ by plane number from the shower start in the longitudinal and transverse directions for leading showers in the $\nu_{e}$ candidate events . . .

13.10A fit to $\theta_{13}$ and $\mathrm{CP}$ violation phase, $\delta$, based on the observation of 6 events in the $\nu_{e}$ appearance channel . . . . . . . . . . . . . 193

13.11-2 $\Delta \log L$ as a function of $\delta$ with $\sin ^{2} 2 \theta_{13}$ fixed at the reactor value of

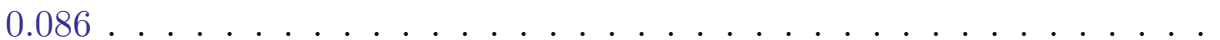

13.12Critical value of $\chi^{2}$ in $\sin ^{2} 2 \theta_{13}$ vs $\delta$ space for normal and inverted hierarchies. . . . . . . . . . . . . . . . . 196

13.13Feldman-Cousins contours for $68 \%$ and $90 \%$ confidence interval in $\theta_{13}$ and $\delta$ space, based on the observation of 6 events in the $\nu_{e}$ appearance

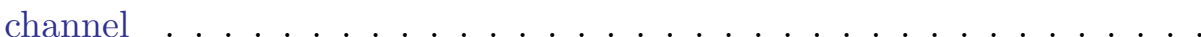




\section{Chapter 1}

\section{Neutrino Physics}

In 1933, three years after Pauli postulated the existence of the neutrino, a particle that he feared "can not be detected", Enrico Fermi proposed a theory of $\beta$ decay which involved the interaction of four fermions at a single point in space. One of these was a massless neutrino. Fortunately, neutrinos only proved to be elusive, but not undetectable. They were experimentally observed in inverse $\beta$ decay interactions, in an experiment led by Cowan and Reines in 1956. And experimental work over the next half century revealed that they are not massless either.

In the following sections we review how neutrinos fit into the current Standard Model of Particle Physics, focusing on the Electroweak sector, since leptons are altogether indifferent to the Strong interaction.

\subsection{Neutrinos in the Standard Model}

The Standard Model of Particle Physics consists of six leptons, which are naturally grouped into three pairs, such that each charged lepton has a neutrino partner:

$$
\left(\begin{array}{c}
e \\
\nu_{e}
\end{array}\right) \quad\left(\begin{array}{c}
\mu \\
\nu_{\mu}
\end{array}\right) \quad\left(\begin{array}{c}
\tau \\
\nu_{\tau}
\end{array}\right)
$$


Neutrinos, in the original Standard Model model are massless particles that are neutral to the electromagnetic and strong forces, and interact only via the weak force. The flavor of neutrinos is dynamically defined by the lepton that pairs with them in the $W^{ \pm}$ mediated charged current weak interactions. For instance, the neutrino that is produced in the weak decay of a charged pion to a muon (eg: $\left.\pi^{-} \rightarrow \mu^{-}+\bar{\nu}_{\mu}\right)$ is a muon flavored (anti-)neutrino.

\subsubsection{Weak Interaction}

To account for the experimentally observed parity violation in weak interactions, the weak current is given a $V-A$ structure and written as $j^{\mu} \sim \bar{\psi}_{l} \gamma^{\mu}\left(1-\gamma^{5}\right) \psi_{\nu}+$ h.c. where $\psi_{l}$ and $\psi_{\nu}$ are the charged lepton and the partner neutrino's wave-functions. As a consequence of this, the charged-current weak force only couples to $\psi_{L}$, while $\psi_{R}$ is eliminated from this interaction. Here, $\psi_{L}$ and $\psi_{R}$ are the left and the right chiral projections of the fermion field:

$$
\psi_{L}=\frac{1-\gamma_{5}}{2} \psi, \quad \psi_{R}=\frac{1+\gamma_{5}}{2} \psi
$$

This also indicates that a left-handed charged lepton and its left-handed partner neutrino form a weak isospin doublet, with $z$-projection, $I_{z}^{W}\left(\nu_{L}\right)=1 / 2$ and $I_{z}^{W}\left(e_{L}\right)=$ $-1 / 2$, where $I^{W}$ denotes the weak isospin.

The right-handed components in this model are iso-singlets, with $I^{W}=0$. Neutral current weak interactions do couple with left as well right-handed particles. However,

the coupling of the right-handed particles to the $Z^{0}$ is $-Q \sin ^{2} \theta_{W}$, where $\theta_{W}$ is the Weinberg angle and $Q$ is the electric charge, which for neutrinos is 0 . Therefore, the right-handed neutrino is neutral to the weak neutral current interaction as well. 


\subsubsection{Free Neutrinos}

The Dirac Lagrangian for a free massless fermion is:

$$
L=\bar{\psi} i \gamma^{\mu} \partial_{\mu} \psi
$$

which yields the Weyl equation of motion:

$$
i \gamma^{\mu} \partial_{\mu} \psi=0
$$

Using the Weyl basis and representation of the $\gamma$ matrices, the above equation decouples into two independent equations,

$$
\begin{aligned}
& E \psi_{L}=-\vec{\sigma} \cdot \vec{p} \psi_{L} \\
& E \psi_{R}=+\vec{\sigma} \cdot \vec{p} \psi_{R}
\end{aligned}
$$

Equations 1.5 show that $\psi_{L}$ is the negative helicity state, with the spin aligned in the direction opposite to the particle's momentum, and vice versa for $\psi_{R}$. Thus, in the absence of mass, helicity coincides with chirality, and is a Lorentz invariant property of the particle. The left and the right chirality states are independent.

For particles with non-zero mass, the Dirac Lagrangian has this additional mass term

$$
m \bar{\psi} \psi=m\left(\bar{\psi}_{L} \psi_{R}+{\overline{\psi_{R}}}_{\psi_{L}}\right)
$$

The Dirac mass term always connects the opposite chirality states of a fermion field. If either $\psi_{L}$ or $\psi_{R}$ field does not exist, the Dirac mass of the particle is automatically 0 . Conversely, if the mass of a particle is 0 , it is not necessary for both $\psi_{L}$ and $\psi_{R}$ to exist.

Therefore, the right-handed neutrino is neutral to all standard model forces, and combined with the assumption of masslessness of neutrinos, has no bearing on observable

phenomena. It is for these reasons that it does not appear in the Standard Model of Particle Physics. 


\subsection{Extending the Standard Model}

It is apparent from the previous section that attributing a Dirac mass to neutrinos requires the existence of a right-handed neutrino. Mass in the Standard Model is generated by interaction with the Higgs field, $m_{D}{ }^{1}=C_{\nu}\langle v\rangle / \sqrt{2}$, where $\langle v\rangle$ is the vacuum expectation value of the Higgs field, and $C_{\nu}$ is the Yukawa coupling of the neutrino field to the Higgs. Since neutrino masses are very small, the coupling $C_{\nu}$ is aberrantly small when compared to couplings of other Standard Model particles to the Higgs, a feature that warrants some theoretical explanation. A popular model for doing so is to introduce a Majorana mass for the neutrinos.

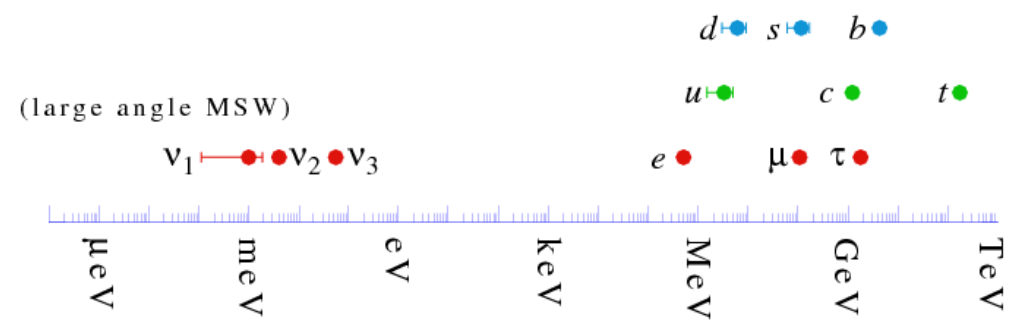

Figure 1.1: Masses of fundamental particles in the Standard Model. Neutrino masses are anomalously small when compared to other particles. [5]

\subsubsection{Majorana Mass of Neutrinos}

Electrically neutral fermions can either be Dirac particles, in which case the particle and its anti-particle are distinct from each other, or they may be Majorana particles. If neutrinos are Majorana fields then, $\psi^{c}$, neutrino's charge-conjugate field, is the same as the neutrino field, except with opposite chirality. In addition to $\bar{\psi} \psi$ term, a Majorana field has other possibilities for constructing mass terms, such as $\bar{\psi}^{c} \psi$ and $\bar{\psi} \psi^{c 2}$.

\footnotetext{
${ }^{1}$ The subscript $D$ is to distinguish the Dirac mass due to interaction with the Higgs field from the Majorana mass discussed in the next section.

${ }^{2}$ Note that these additional terms violate lepton number conservation, since $\Delta L$ for them is 2 .
} 
Enumerating all the possible mass terms allowed for neutrinos results in the following mass term in the Lagrangian:

$$
L_{m}=\frac{1}{2}\left(m_{D} \bar{\psi}_{R} \psi_{L}+m_{D}{\overline{\psi_{R}}}_{R}^{c} \psi_{L}^{c}+m_{L}{\overline{\psi_{L}}}^{c} \psi_{L}+m_{R}{\overline{\psi_{R}}}^{c} \psi_{R}\right)+\text { h.c. }
$$

which can be represented more compactly as follows:

$$
\left(\begin{array}{cc}
\bar{\psi}_{L}^{c} & \psi_{R}^{c}
\end{array}\right)\left(\begin{array}{ll}
m_{L} & m_{D} \\
m_{D} & m_{R}
\end{array}\right)\left(\begin{array}{l}
\bar{\psi}_{L} \\
\psi_{R}
\end{array}\right)
$$

This suggests that the left and the right chiral components of neutrino flavor eigenstates do not have a definite mass because of the existence of the off-diagonal Dirac mass. Diagonalizing this matrix reveals the masses of the neutrino mass eigenstates as

$$
m_{1,2}=\frac{1}{2}\left(\left(m_{L}+m_{R}\right) \pm \sqrt{\left(m_{L}-m_{R}\right)^{2}-4 m_{D}^{2}}\right)
$$

$m_{L}$ is required to be 0 in the standard model . This is because $\psi_{L}$ destroys a lefthanded neutrino with weak isospin $1 / 2$ and ${\overline{\psi_{L}}}^{c}$ creates a right-handed neutrino with weak isospin $-1 / 2$ which would require a Higgs field with weak isospin of 1 . If we assume that $m_{R}$ is very large $\left(m_{R}>>m_{D}\right)$, then the mass eigenvalues simplify as

$$
\begin{aligned}
& m_{1} \sim \frac{m_{D}^{2}}{m_{R}} \\
& m_{2} \sim m_{R}\left(1+\frac{m_{D}^{2}}{m_{R}^{2}}\right) \sim m_{R}
\end{aligned}
$$

The mass $m_{1}$ is suppressed by $1 / m_{R}$, so if $m_{R}$, and therefore $m_{2}$, is very large, $m_{1}$ becomes very small. This is known as the see-saw mechanism and provides a natural explanation for the smallness of the observed neutrino masses, in comparison with other Standard Model fermions. For instance, if $m_{D}$ is $O(M e V)$, as it is for other charged leptons, and $m_{R} \sim 10^{15} \mathrm{eV}, m_{1}$ is in the $m e V$ range, consistent with current limits.

In summary, if we need to introduce a Dirac mass $m_{D}$ for neutrinos in the Standard Model Lagrangian, we are forced to introduce a sterile right-handed neutrino, which 
has no known interaction with matter (except with Higgs). If neutrinos are Majorana particles, they are their own anti-particles. This possibility violates lepton number conservation, but provides a mechanism to explain the smallness of neutrino masses in relation to the others.

\subsection{Neutrino Oscillations}

What convinces us of the need to include neutrino masses in the Standard Model is the observation of neutrino oscillation. The quantum mechanical phenomenon of oscillation occurs when the state that a particle is produced in is not a mass eigenstate. In the case of neutrinos, the state they are produced in is a weak flavor eigenstate while the state that propagates is an eigenstate of the free neutrino Hamiltonian, ie, the mass eigenstate.

\subsubsection{Oscillations in Vacuum}

A neutrino with flavor $l$ at time $t=0$ can be expressed in terms of the neutrino mass eigenstates $\nu_{j}$ as follows:

$$
\left|\nu_{\alpha}(0)\right\rangle=\sum_{j=0}^{3} U_{\alpha j}\left|\nu_{j}(0)\right\rangle
$$

where $U_{\alpha j}$ are elements of a $3 \times 3$ unitary matrix that encodes the mixing parameters and is known as the Pontecorvo-Maki-Nakagawa-Sakata or PMNS matrix [6]. At a later time $t$, the above state evolves to

$$
\left|\nu_{\alpha}(t)\right\rangle=\sum_{j=0}^{3} U_{\alpha j}\left|\nu_{j}(t)\right\rangle=\sum_{j=0}^{3} U_{\alpha j} e^{-i E_{j} t}\left|\nu_{j}(0)\right\rangle
$$

$E_{j}$ are the energies of the individual mass states. If we assume neutrino masses to be small in comparison to their total energy,

$$
E_{j}=\sqrt{p_{j}^{2}+m_{j}^{2}} \simeq E_{j}\left(1+\frac{m_{j}^{2}}{2 E_{j}^{2}}\right) \simeq E\left(1+\frac{m_{j}^{2}}{2 E^{2}}\right)
$$


Since neutrinos are ultra-relativistic, $t \sim L$, where $L$ is the propagation distance of the neutrino, or in the language neutrino oscillation experiments, the baseline. Therefore, the probability of transition of the neutrino that began its life in flavor state $\alpha$, to a flavor state $\beta$ is given by:

$$
\begin{aligned}
P_{\alpha \rightarrow \beta}(E, L) & =\left|\left\langle\nu_{\beta} \mid \nu_{\alpha}(t)\right\rangle\right|^{2} \\
& =\left|\sum_{j} e^{-i \frac{m_{j}^{2} L}{2 E}} U_{\beta j}^{*} U_{\alpha j}\right|^{2}
\end{aligned}
$$

For the case of only two neutrino generations, the oscillation probability reduces to this simpler form:

$$
P_{\alpha \rightarrow \beta}(E, L)=\sin ^{2} 2 \theta \sin ^{2}\left(\frac{\Delta m^{2} L}{4 E}\right)
$$

since $U$ is just the $2 D$ rotation matrix,

$$
U=\left(\begin{array}{cc}
\cos \theta & \sin \theta \\
-\sin \theta & \cos \theta
\end{array}\right)
$$

$\theta$ is the angle with which the mass and the flavor bases are rotated with respect to one another and $\Delta m^{2}$ is the mass-squared difference, $m_{2}^{2}-m_{1}^{2}$. This simple analysis highlights a few important features of neutrino oscillations: oscillation probability is dependent on the ratio of the distance the neutrinos travel ( $L$, baseline) to the neutrino energy (E), the mass squared difference of the mass states and $\theta$, the angle by which the mass basis is rotated with respect to the weak basis for neutrinos. The $\sin ^{2}\left(\frac{\Delta m^{2}}{4} \frac{L}{E}\right)$ term is what ascribes the oscillatory character to this phenomenon.

If the mixing angle, $\theta$ or the mass-squared difference, $\Delta m^{2}$ is zero, oscillations would not be observed. Thus, for neutrino oscillations to be an observable effect, not only must neutrinos have mass, but the masses must also be non-degenerate.

Generalizing the 2-flavor oscillations to 3 -flavors, we pick up two additional rotation angles, bringing the total to $3: \theta_{13}, \theta_{23}$ and $\theta_{12}$, and a complex phase, $\delta$. The full $3 \times 3$ PMNS matrix is conventionally expressed as: 


$$
\left(\begin{array}{ccc}
c_{13} c_{12} & c_{13} s_{12} & s_{13} e^{-i \delta} \\
-c_{23} s_{12}-s_{13} s_{23} c_{12} e^{i \delta} & c_{23} c_{12}-s_{13} s_{23} s_{12} e^{i \delta} & c_{13} s_{23} \\
s_{23} s_{12}-s_{13} c_{23} c_{12} e^{i \delta} & -s_{23} c_{12}-s_{13} c_{23} s_{12} e^{i \delta} & c_{13} c_{23}
\end{array}\right)
$$

where $c_{i j}$ and $s_{i j}$ are the cosine and sine of the mixing angle $\theta_{i j}$.

The oscillation probability, considering three flavors is:

$$
\begin{aligned}
P_{\alpha \rightarrow \beta}(E, L)= & \delta_{\alpha \beta}-4 \sum_{j>k} \Re\left(U_{\beta j}^{*} U_{\beta k} U_{\alpha j} U_{\alpha k}^{*}\right) \sin ^{2}\left(\frac{\Delta m_{j k}^{2} L}{4 E}\right)+ \\
& 2 \sum_{j>k} \Im\left(U_{\beta j}^{*} U_{\beta k} U_{\alpha j} U_{\alpha k}^{*}\right) \sin \left(\frac{\Delta m_{j k}^{2} L}{2 E}\right)
\end{aligned}
$$

Where $\Delta m_{j k}^{2}$ is $m_{j}^{2}-m_{k}^{2}$. If the mixing matrix $U$ is real, ie $\delta=0$, the second term in the oscillation probability is zero and oscillations are time-reversible, that is, $P_{\alpha \rightarrow \beta}=P_{\beta \rightarrow \alpha}$ for $\alpha \neq \beta$. Since $C P T$ is observed to be an exact symmetry, a non-zero value of $\delta$ implies breaking of $T$ symmetry and therefore, the violation of $C P$ symmetry. For this reason, $\delta$ is termed the $C P$-violating phase.

\subsubsection{Oscillations in Matter}

In realistic experiments, neutrinos pass through matter, and not through vacuum, and in doing so, interact with it. Interactions with matter that maintain the coherence of the neutrino state allow for interference between the scattered and the unscattered wavefunctions and result in observable effects in neutrino oscillations. Incoherent interactions are a negligible effect due to the smallness of neutrino interaction cross-sections. Therefore, the only interactions that are interesting are forward elastic scattering of neutrinos on protons, neutrons and electrons that constitute matter. This section presents the correction to the free neutrino Hamiltonian due to the presence of matter. We closely follow Ho-Kim's treatment of the problem in [7].

The $Z^{0}$ mediated neutral current interactions on $p, n$ and $e$ are identical for $\nu_{e}, \nu_{\mu}$ and $\nu_{\tau}$. However, only $\nu_{e}$ can have $W$ mediated elastic charged-current interaction on 


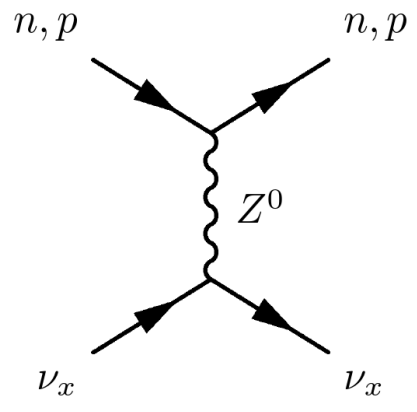

Neutral Current

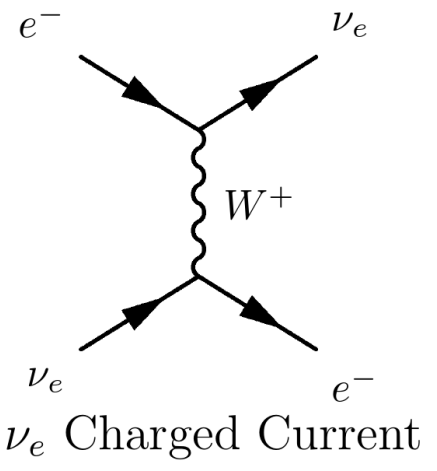

Figure 1.2: Allowed coherent scattering modes for neutrinos passing through the earth matter. The neutral current modes are accessible to all neutrino flavors, while the charged current mode is open only to electron flavored neutrinos in normal matter.

$e^{-}$, since normal matter does not contain $\mu$ or $\tau$. These interactions result in corrections to the free-neutrino Hamiltonian of the form:

$$
\begin{aligned}
& V_{N C}=-G_{F} N_{n} / \sqrt{2} \\
& V_{C C}=G_{F} N_{e} \sqrt{2}
\end{aligned}
$$

Here, $G_{F}$ is the Fermi constant, and $N_{n}$ and $N_{e}$ are neutron and electron densities. The neutral current contributions due to scattering on protons and electrons cancel exactly for electrically neutral matter, hence neutron density alone determines the potential. The total energy of the neutrino becomes $E^{2}=(p+V)^{2}+m^{2} \simeq p^{2}+m^{2}+2 p V$, since $V$ is very small. This can be viewed as a modification to the neutrino mass, $m^{2} \rightarrow m^{2}+2 p V$

The equation of motion for free-neutrinos in vacuum in the two flavor case is

$$
\begin{aligned}
i \frac{d}{d t}\left(\begin{array}{l}
\nu_{1} \\
\nu_{2}
\end{array}\right) & =H\left(\begin{array}{c}
\nu_{1} \\
\nu_{2}
\end{array}\right) \\
& =\left[E+\left(\begin{array}{cc}
m_{1}^{2} / 2 E & 0 \\
0 & m_{2}^{2} / 2 E
\end{array}\right)\right]\left(\begin{array}{l}
\nu_{1} \\
\nu_{2}
\end{array}\right)
\end{aligned}
$$


To transform this to the flavor basis, $H \rightarrow H^{\prime}=U H U^{\dagger}$,

$$
\begin{aligned}
i \frac{d}{d t}\left(\begin{array}{c}
\nu_{e} \\
\nu_{\mu}
\end{array}\right) & =H^{\prime}\left(\begin{array}{c}
\nu_{e} \\
\nu_{\mu}
\end{array}\right) \\
& =\left[E+\frac{m_{1}^{2}+m_{2}^{2}}{4 E}+\frac{\Delta m^{2}}{4 E}\left(\begin{array}{cc}
-\cos 2 \theta & \sin 2 \theta \\
\sin 2 \theta & \cos 2 \theta
\end{array}\right)\right]\left(\begin{array}{l}
\nu_{e} \\
\nu_{\mu}
\end{array}\right)
\end{aligned}
$$

Correcting the free Hamiltonian for interactions with matter adds $V_{N C}$ to the effective masses of $\nu_{e}$ as well as $\nu_{\mu}$. $V_{C C}$, however, adds only to the mass of $\nu_{e}$ and therefore adds to $H_{00}^{\prime}$ element of the mixing matrix. Diagonalizing this new matrix leads to corrections to the vacuum $\Delta m^{2}$ and mixing angle $\theta$.

$$
\begin{aligned}
\sin 2 \theta_{\text {mat }} & =\frac{\sin 2 \theta}{A_{\text {mat }}} \\
\Delta m_{\text {mat }}^{2} & =\Delta m^{2} A_{\text {mat }} \\
\text { where, } \quad A_{\text {mat }} & =\sqrt{\left(\frac{2 E V_{C C}}{\Delta m^{2}}-\cos 2 \theta\right)^{2}+\sin ^{2} 2 \theta}
\end{aligned}
$$

The form of $A_{\text {mat }}$ indicates a resonance behavior for

$$
\begin{aligned}
V_{C C} & =\sqrt{2} G_{F} N_{e}=\frac{\Delta m^{2} \cos 2 \theta}{2 E} \\
\Longrightarrow N_{r e s} & =\frac{\Delta m^{2} \cos 2 \theta}{2 \sqrt{2} G_{F} E}=6.56 \times 10^{3} \frac{\Delta m^{2}\left[\mathrm{eV}^{2}\right]}{E[\mathrm{GeV}]} \cos 2 \theta N_{A}
\end{aligned}
$$

That is, at certain electron densities, matter effect causes even small mixing angles to become nearly $45^{\circ}$, resulting in an amplification in oscillation probabilities. This effect is known as the Mikheyev-Smirnov-Wolfenstein or $M S W$ effect. It can be a significant effect where neutrinos pass through a very dense medium, for instance the core of a star.

Even for neutrinos passing through earth crust where $N_{e} \sim 1.3 N_{A}^{3} / \mathrm{cm}^{3}$ [8] matter effects can play a crucial role. For instance, for $\Delta m^{2} \sim 10^{-3} \mathrm{eV}^{2}$ and neutrino energies $\sim 1 \mathrm{GeV}$, a vacuum mixing angle $\theta=10^{\circ}$ becomes $\sim 12^{\circ}$ in matter. The oscillation probability depends on $\sin ^{2} 2 \theta$, which changes from $\sim 0.34$ to $\sim 0.42$, a $\sim 20 \%$ effect.

\footnotetext{
${ }^{3} N_{A}$ is the Avogadro number, $6.023 \times 10^{23}$
} 


\section{Summary}

Neutrinos, that were thought to be massless, undetectable particles at the time of their conception, have proved to be neither. Neutrino oscillations require neutrinos to have non-zero masses and are one of the few signs of physics beyond the Standard Model. Neutrino masses are known to be very small $(<e V)$ compared to other Standard Model fermions which might indicate that their nature is different from other SM particles. In particular, the possibility of neutrinos having a Majorana mass has been discussed here which provides a natural explanation for the smallness of the observed neutrino masses.

Oscillations are an interesting way of probing the physics of neutrino masses, which is largely inaccessible otherwise, due to their small masses. The next chapter lays out the current state of knowledge of the neutrino oscillation parameter space and the experiments instrumental in making some of the measurements. 


\section{Chapter 2}

\section{Status of Neutrino Oscillation Measurements}

In modern experiments, neutrinos continue to pose a difficulty in detection as they only interact rarely, via the weak interaction. While they are regularly produced in collisions at collider experiments, they escape undetected and are more or less dismissed as "missing energy".

However, the discovery of neutrino oscillations has led to a rich program in experimental neutrino physics in the last three decades. Neutrino oscillation measurements have been made on a variety of neutrino sources, such as solar and atmospheric neutrinos, and reactor and accelerator neutrinos. These sources produce neutrinos of different flavors and in different energy regimes, making them sensitive to different oscillation parameters.

\subsection{Conventions and Nomenclature}

In the three neutrino flavor scenario, there are two independent mass-squared differences constructed from the three mass eigenvalues $m_{1}, m_{2}$ and $m_{3}$. The third degree of freedom is the sign of one of the mass-squared differences. The convention is to set $m_{1}$ 
to be smaller than $m_{2}$, ie $\Delta m_{21}^{2}=m_{2}^{2}-m_{1}^{2}$ is positive definite.

The analysis of the oscillation experiments data indicated that one mass-squared difference is much smaller than the other $\left(\Delta m_{21}^{2} \simeq 3 \times 10^{-2} \times \Delta m_{32}^{2}\right)$. This means that the $L / E$ factor at which an experiment is sensitive to $\Delta m_{21}^{2}$, it is insensitive to $\Delta m_{32}^{2}$, and 2-flavor oscillation formalism provides a good description of the phenomenology to within a few percent. The angle and mass-squared difference that govern the solar oscillations to leading order are $\theta_{12}$ and $\Delta m_{21}^{2}$. The atmospheric oscillation parameters then are $\Delta m_{32}^{2}$ and $\theta_{23}$. This leaves $\theta_{13}$, which controls the amplitude of the electronneutrino disappearance channel in reactor neutrino experiments.

\subsection{Oscillation Parameter Measurements}

\subsection{1 $\theta_{12}$ and $\Delta m_{21}^{2}$ Measurements}

The nuclear chain reactions that occur in the core of the sun produce a large flux of electron flavored neutrinos. The energy spectrum of neutrinos depends upon the core temperature, composition of the sun, cross-sections of nuclear reactions involved, opacity of the sun, etc. These factors were studied in detail by Bahcall and collaborators, and encapsulated in the Standard Solar Model (SSM). The model, in addition to other measurables, predicts a solar neutrino spectrum that can be observed on earth. Most of these neutrinos, close to $91 \%$, are produced in proton-proton fusion reactions:

$$
p+p \rightarrow d+e^{+}+\nu_{e}
$$

However, the maximum energy of the neutrinos produced in this interaction is $0.4 \mathrm{MeV}$, making detection very challenging. Higher energy neutrinos, with maximum energy of $14 \mathrm{MeV}[9]$ are produced in the decay of Boron in the sun and contribute to less than 
$1 \%$ of the total flux:

$$
\begin{aligned}
& d+p \rightarrow{ }^{3} \mathrm{He}+\gamma \\
& { }^{3} \mathrm{He}+{ }^{3} \mathrm{He} \rightarrow{ }^{4} \mathrm{He}+2 p \\
& { }^{3} \mathrm{He}+{ }^{4} \mathrm{He} \rightarrow{ }^{7} \mathrm{Be}+\gamma \\
& { }^{7} \mathrm{Be}+p \rightarrow{ }^{8} \mathrm{~B}+\gamma \\
& { }^{8} \mathrm{~B} \rightarrow{ }^{8} \mathrm{Be}+e^{+}+\nu_{e}
\end{aligned}
$$

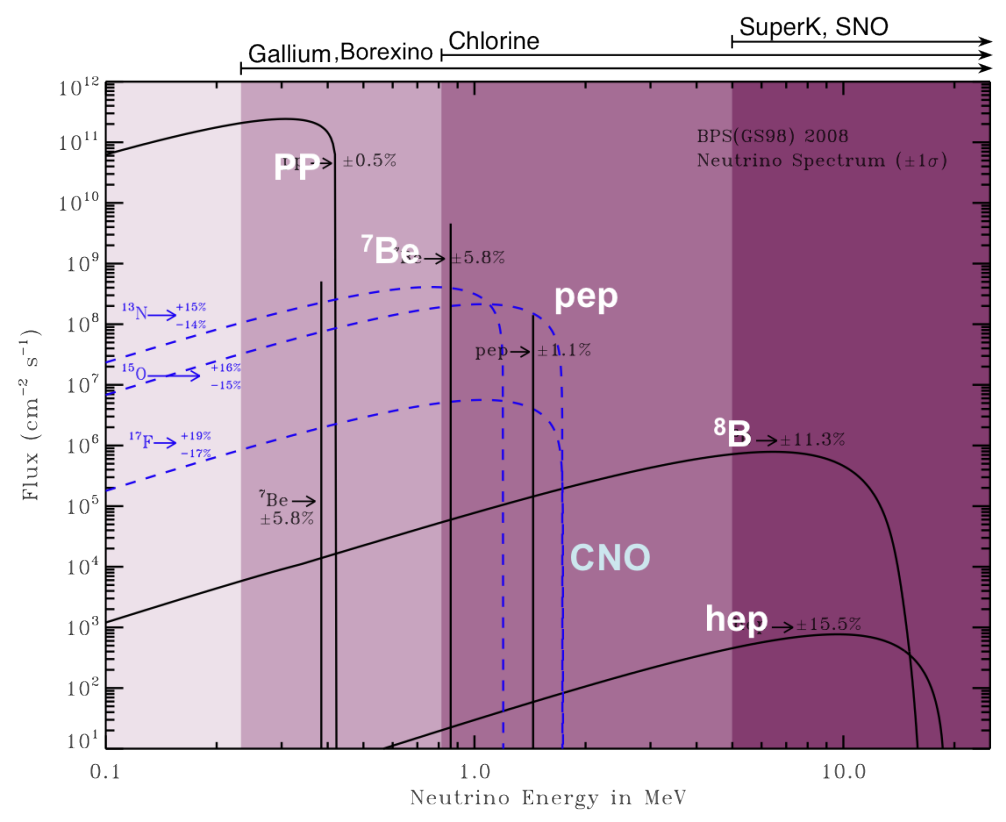

Figure 2.1: Predicted solar neutrino spectrum [1]

The first experiment to directly detect solar neutrinos was conducted by Raymond Davis Jr. and collaborators [10], using electron neutrino capture on ${ }^{37} \mathrm{Cl}$ to produce ${ }^{37} \mathrm{Ar}$ in liquid $\mathrm{C}_{2} \mathrm{Cl}_{4}$ at the Homestake Mine in South Dakota. Helioseismological measurements are in good agreement with the SSM [11], but the solar neutrino observation experiments have consistently measured a neutrino flux significantly below the SSM prediction. An interesting, chronological account of the unravelling of this so-called Solar 
Neutrino Problem can be found in [12]. The solution to the problem is now understood in the context of neutrino oscillations.

A series of solar neutrino detection experiments, such as GALLEX, GNO and SAGE [13][14][15], used neutrino absorption on Gallium for detection and observed similar deficits. Gallium has a threshold of $233 \mathrm{keV}$ and therefore, enabled observation of $\nu_{e}$ resulting from $p-p$ fusion. The resolution, however, came from the Sudbury Neutrino Observatory (SNO), a heavy water Cherenkov experiment in Canada, that measured the rate of both, the $\mathrm{NC}$ and $\mathrm{CC}$ interactions of solar neutrinos resulting from Boron decay:

$$
\begin{aligned}
& \nu_{e}+d \rightarrow e^{-}+p+p \\
& \nu+d \rightarrow \nu+p+n
\end{aligned}
$$

The measurement of the flavor agnostic neutral current interactions made the experiment sensitive to the total neutrino flux, independent of flavors. Their sensitivity to the $\nu_{e}$ charged current interaction allowed them to measure the flux of electron-flavored neutrinos alone. They observed that the total, flavor-independent neutrino flux was in agreement with the SSM prediction, while the $\nu_{e}$ flux was only $1 / 3$ of the predicted value [16].

While travelling from the core of the sun to the surface, the electron neutrinos encounter, to a good approximation, exponentially varying density of electrons. In the core, for the higher energy neutrinos $(E>4-5 \mathrm{MeV})$, the electron density is close to the resonance density, $N_{\text {res }}$, discussed in the previous chapter. The $\nu_{e}$ state transitions to mostly $\nu_{2, \text { mat }}$ state, ie the heavier mass state in matter, in such conditions. If the electron density in the sun changes adiabatically, the state $\nu_{2, \text { mat }}$ smoothly transitions 
to $\nu_{2}$, the heavier mass state in vacuum, at the surface of the sun. Given that

$$
\begin{aligned}
& \left|\nu_{2}\right\rangle=\sin \theta\left|\nu_{e}\right\rangle+\cos \theta\left|\nu_{x}\right\rangle \\
& P\left(\nu_{e} \rightarrow \nu_{e}\right)=\sin ^{2} \theta
\end{aligned}
$$

For neutrinos with energies less than $4,5 \mathrm{MeV}, \theta_{\text {mat }} \simeq \theta$, and $P\left(\nu_{e} \rightarrow \nu_{e}\right)=1-\frac{1}{2} \sin ^{2} \theta$. The best fit of current data from low and higher energy solar neutrino experiments results in a value of $\theta_{12} \sim 33.4^{\circ}$ and $\Delta m_{21}^{2} \sim 7.53 \times 10^{-5} \mathrm{eV}^{2}$. The $\theta_{12}$ angle is a measure of the overlap of the $\nu_{e}$ flavor state with $\nu_{2}$. So these results mean that the mass state $\nu_{1}$ is about $2 / 3 \nu_{e}$ and $\nu_{2}$ is $\sim 1 / 3 \nu_{e}$.

\subsection{2 $\theta_{23}$ and $\left|\Delta m_{32}^{2}\right|$ Measurements}

Collision of cosmic rays with nuclei in the earth's atmosphere produce showers of hadrons. Pions being the lightest hadrons, are produced in large numbers and their decay to muons produces muon neutrinos. The resulting muons further decay to produce an electron and a muon neutrino:

$$
\begin{aligned}
& \pi^{+} \rightarrow \mu^{+}+\nu_{\mu} \\
& \mu^{+} \rightarrow e^{+}+\nu_{e}+\bar{\nu}_{\mu}
\end{aligned}
$$

From this decay chain, the ratio of muon flavored to electron flavored neutrinos is expected to be 2:1. Various experiments had evidence of atmospheric neutrino oscillations before Super Kamiokande (Super K) established it statistically [17]. What stands the Super K experiment apart from its predecessors is that the detector is a massive 50 kiloton water Cherenkov tank instrumented with photo-multiplier tubes. The Cherenkov rings produced by energetic particles passing through the detector allow real-time event energy and angle reconstruction. The angle reconstruction was especially important in building confidence that the neutrinos were indeed atmospheric. The collaboration recorded the $\nu_{\mu}$ and $\nu_{e} \mathrm{CC}$ interactions as a function of the zenith-angle, and found the rate of $\nu_{\mu} \mathrm{CC}$ interactions due to neutrinos coming from below (having passed through 
the earth) was signicantly lower than the rate of those coming from above. No such discrepancy was observed in the $\nu_{e} \mathrm{CC}$ rates. The leading interpretation was that the disappearance of $\nu_{\mu}$ was predominantly due to $\nu_{\mu} \rightarrow \nu_{\tau}$ transition.

The accelerator neutrino beams are obtained in much the same way as the atmospheric neutrinos, except that the pions are produced by colliding energetic protons on a stationary target. The K2K experiment in Japan and MINOS experiment at Fermilab, USA, used such beams of muon neutrinos to observe $\nu_{\mu}$ disappearance. The neutrinos produced in this manner have energies of $O(G e V)$ and require baselines of 100's of kilometers. These beams of neutrinos travel through the earth's crust and are sensitive to matter effects.

The current best fit value of the global data is $\Delta m_{32}^{2} \sim 2.44 \times 10^{-3} \mathrm{eV}^{2}$ (normal hierarchy) and $\sin ^{2} 2 \theta_{23} \sim 1$.

\subsection{3 $\quad \theta_{13}$ Measurements}

Nuclear power reactors are powerful sources of low energy electron anti-neutrinos, with average energy of about 3 to $4 \mathrm{MeV}$. By choosing a baseline of about $1 \mathrm{~km}$, the experiments are sensitive to $\left|\Delta m_{32}^{2}\right|$ governed $\nu_{e}$ disappearance channel. At these $L / E$ values, the oscillation terms due to $\Delta m_{21}^{2}$ may be ignored to a good approximation. The amplitude of this oscillation is dependent on the mixing-angle $\theta_{13}$, and the $\bar{\nu}_{e}$ survival probability in the two-flavor approximation is:

$$
P\left(\overline{\nu_{e}} \rightarrow \overline{\nu_{e}}\right) \simeq 1-\sin ^{2} 2 \theta_{13} \sin ^{2}\left(\frac{\Delta m_{32}^{2} L}{4 E}\right)
$$

$\theta_{13}$ is the smallest and the last neutrino mixing-angle to be measured. It parameterizes the size of the $\nu_{e}$ component in the $m_{3}$ mass state. CHOOZ was the first to make this measurement using reactor neutrinos and they measured $\sin ^{2} 2 \theta_{13}<0.15$ [18], ie their measurement was consistent with zero. A non-zero value of $\theta_{13}$ is crucial to the measurement of $\mathrm{CP}$ violation in neutrinos (see equation 1.15). 
The first results reporting evidence of non-zero value of $\theta_{13}$ were announced in 2012 by Double CHOOZ [19] in France, Daya Bay in China [20] and RENO [21] in Korea. The three detectors are very similar. They contain Gadolinium doped liquid scintillator that produces a flash of light due to annihilation of a positron resulting from antineutrino absorption on a proton $\left(\bar{\nu}_{e}+p^{+} \rightarrow e^{+}+n^{0}\right)$. The free neutron thermalizes and is eventually absorbed by a Gd nucleus, producing a delayed signal. The coincidence of the prompt and delayed signals help reject backgrounds efficiently. The Daya Bay experiment, which consists of 8 detectors in the vicinity of 6 commercial nuclear power reactors has the world's most precise measurement of this parameter.

Higher statistics analyses from the reactor experiments have since been published. The current global fit value is $\theta_{13} \sim 8.9^{\circ}$. The $\theta_{13}$ parameter also controls the amplitude of the leading term in the $\nu_{\mu}$ to $\nu_{e}$ appearance channel. $\mathrm{T} 2 \mathrm{~K}$ and $\mathrm{NO} \nu \mathrm{A}$ are the leading experiments in this channel which is discussed in more detail later.

\subsection{Unknown Parameters}

\subsubsection{Mass Hierarchy}

As hinted before, oscillation experiments so far have not been able to resolve the sign of $\Delta m_{32}^{2}$ and $\Delta m_{31}^{2}$. Neutrino oscillation experiments tell us that $\Delta m_{21}^{2} \simeq 3 \times 10^{-2} \times$ $\Delta m_{32}^{2}$. Therefore, if $\left|\Delta m_{32}^{2}\right|$ is positive, $m_{3}>>m_{2}, m_{1}$, ie there are two light neutrinos and one heavier neutrino, a scenario known as Normal Hierarchy $(\mathrm{NH})$. If the sign is negative, we have two heavier neutrinos, and a lighter one. This scenario is called Inverted Hierarchy ${ }^{1}$. The vacuum oscillation probabilities only depend on the sinesquared of the mass-squared differences and are not sensitive to the mass hierarchy.

\footnotetext{
${ }^{1}$ Another scenario is that the splittings between neutrino masses are much smaller than the neutrino masses, so that the neutrino masses are degenerate. However, since oscillations are not sensitive to the absolute mass scale, this case is not discussed here.
} 


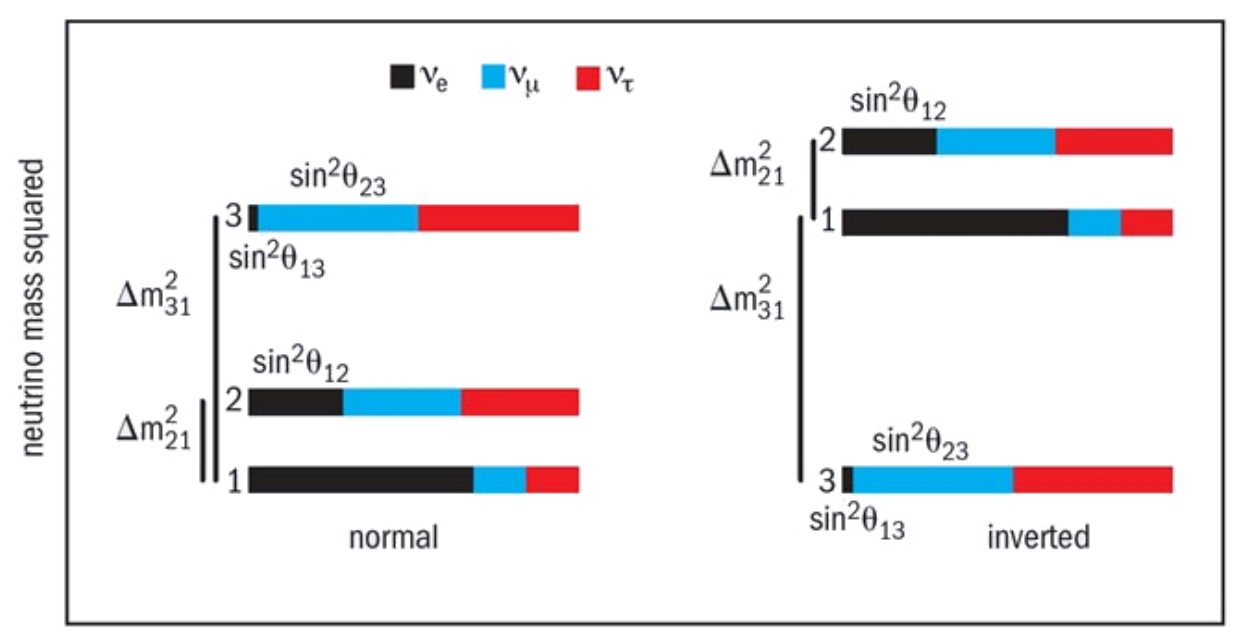

fractional flavour content

Figure 2.2: Mass hierarchy in neutrinos

The normal ordering of neutrino masses is considered normal because the electronflavored neutrino state comprises $2 / 3$ of the $\nu_{1}$ mass state, and it is natural to expect the lightest mass state to correspond to the first generation flavor state. The mass hierarchy is also of critical importance in the searches for neutrino-less double-beta (denoted as $0 \nu \beta \beta$ ) decay. The rate of lepton number violating $0 \nu \beta \beta$ depends on the square of $<m_{\beta \beta}>$, the effective Majorana mass of the neutrino.

$$
<m_{\beta \beta}>=\left|\cos ^{2} \theta_{13} \cos ^{2} \theta_{12} m_{1}+e^{i \Delta \alpha_{21}} \cos ^{2} \theta_{13} \sin ^{2} \theta_{12} m_{2}+e^{i \Delta \alpha_{21}} \sin ^{2} \theta_{13} m_{3}\right|^{2}
$$

where $\Delta \alpha_{i 1}$ are physically relevant Majorana phases (see section on neutrino-less double beta decay in [6]). The effective Majorana mass can be computed as a function of the lightest neutrino mass as shown in figure 2.3. At small enough neutrino masses, the rate of $0 \nu \beta \beta$ decay becomes sensitive to the neutrino mass hierarchy. So hierarchy determination would help clarify the results from upcoming $0 \nu \beta \beta$ experiments and help in evaluating the sensitivity and in the design of the next generation of experiments. 


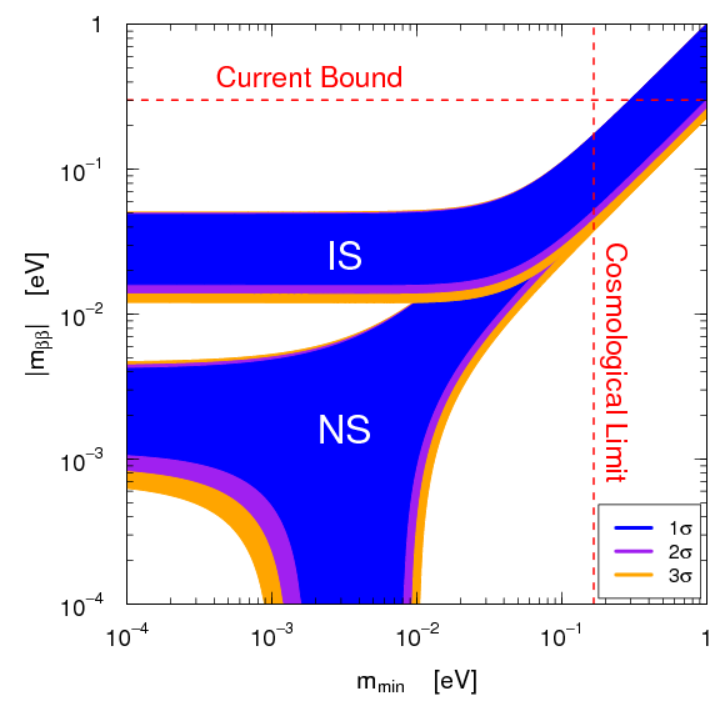

Figure 2.3: Values of effective Majoarana mass, $m_{\beta \beta}$, as a function of the lightest neutrino mass. At small values of neutrino masses, $m_{\beta \beta}$ is sensitive to neutrino mass hierarchy. Plot from [22].

\subsubsection{CP Violation}

The violation of $\mathrm{T}$, and therefore $\mathrm{CP}$ symmetry arises from the complex phase, $\delta$ in the PMNS matrix. If CP is violated, it implies that $P\left(\nu_{\alpha} \rightarrow \nu_{\beta}\right) \neq P\left(\nu_{\beta} \rightarrow \nu_{\alpha}\right)$ for $\alpha \neq \beta$. Since changing the flavor of the source neutrinos is not feasible, experimental searches for $\mathrm{CP}$ violation are carried out by changing the neutrino source to an anti-neutrino source. The size of the difference between the neutrino and anti-neutrino oscillation probabilities can be obtained with the help of equation 1.16:

$$
\begin{aligned}
P\left(\nu_{\alpha} \rightarrow \nu_{\beta}\right)-P\left(\nu_{\beta} \rightarrow \nu_{\alpha}\right) & =P\left(\nu_{\alpha} \rightarrow \nu_{\beta}\right)-P\left(\overline{\nu_{\alpha}} \rightarrow \overline{\nu_{\beta}}\right) \\
& =4 \sum_{j>k} \Im\left(U_{\beta j}^{*} U_{\beta k} U_{\alpha j} U_{\alpha k}^{*}\right) \sin \left(\frac{\Delta m_{j k}^{2} L}{2 E}\right)
\end{aligned}
$$

Note that the above difference is zero for survival probability, $P\left(\nu_{\alpha} \rightarrow \nu_{\alpha}\right)$, because this is a $\mathrm{T}$ invariant and therefore, a $\mathrm{CP}$ invariant process. 


\subsection{3 $\theta_{23}$ Octant}

The current measurements of $\theta_{23}$ are consistent with $45^{\circ} . \theta_{23}$ angle primarily determines the admixture of the mass state $\nu_{3}$. If $\theta_{23}$ is exactly $45^{\circ}$, all mass states contain equal proportions of $\nu_{\mu}$ and $\nu_{\tau}$. This might indicate a symmetry in the neutrino sector that has not yet been accounted for in our theoretical framework. This scenario is known as maximal-mixing. An accurate measurement of the $\nu_{\mu}$ disappearance probability can tell us if the mixing is maximal or non-maximal. But determining the octant of $\theta_{23}$, ie whether $\theta_{23}$ less or more than $45^{\circ}$, from $\nu_{\mu}$ disappearance is difficult because the leading order term in probability of this channel is given by:

$$
P\left(\nu_{\mu} \rightarrow \nu_{\mu}\right) \simeq 1-\sin ^{2} 2 \theta_{23} \sin \left(\frac{\Delta m_{32}^{2} L}{4 E}\right)
$$

That is, the leading term in the $\nu_{\mu}$ disappearance probability depends on $\sin ^{2} 2 \theta_{23}$, which is close to or equal to 1 . The probability is the same for $2 \theta_{23} \lesssim 90^{\circ}$ and $2 \theta_{23} \gtrsim 90^{\circ}$

\section{$2.4 \quad \nu_{\mu} \rightarrow \nu_{e}$ on Long Baseline}

$\nu_{e}$ appearance in a $\nu_{\mu}$ beam on a long baseline is the most promising and relatively easy to access oscillation channel to measure the remaining unknown parameters of neutrino oscillations. The full three-flavor $\nu_{\mu} \rightarrow \nu_{e}$ oscillation probability in vacuum is [23]

$$
\begin{aligned}
P\left(\stackrel{(-)}{\nu}_{\mu} \rightarrow \stackrel{(-)}{\nu}_{e}\right) & =P_{1}+P_{2}+P_{3}+P_{4} \\
P_{1} & =\sin ^{2} 2 \theta_{13} \sin ^{2} \theta_{23} \sin ^{2} \frac{\Delta m_{32}^{2} L}{4 E} \\
P_{2} & =\sin ^{2} 2 \theta_{13} \cos ^{2} \theta_{23} \sin ^{2} \frac{\Delta m_{21}^{2} L}{4 E} \\
P_{3} & =\stackrel{(+)}{-} J \sin \delta \sin \frac{\Delta m_{32}^{2} L}{4 E} \\
P_{4} & =J \cos \delta \cos \frac{\Delta m_{32}^{2} L}{4 E}
\end{aligned}
$$

where

$$
J=\cos \theta_{31} \sin 2 \theta_{13} \sin 2 \theta_{23} \sin 2 \theta_{21} \sin \frac{\Delta m_{32}^{2} L}{4 E} \sin \frac{\Delta m_{21}^{2} L}{4 E}
$$


There are a few features to be noted in this formula:

1. For neutrino energy close to $2 \mathrm{GeV}$, and baseline of $\sim 800 \mathrm{~km},\left|\Delta m_{32}^{2}\right| L / 4 E$ is $\sim \pi / 2$, and $\Delta m_{21}^{2} L / 4 E$ is $\sim 3 \times 10^{-2}$ times smaller than that, so $P_{1}$ is the leading term and $P_{2}$ is negligible.

2. The amplitude of $P_{1} \propto \sin ^{2} 2 \theta_{13}$, that depends on the smallest mixing angle, $\sim 9^{\circ}$, which makes the probability of this transformation small.

3. Importantly, $P_{1} \propto \sin ^{2} \theta_{23}$, which makes the $\nu_{\mu}$ to $\nu_{e}$ transition sensitive to the octant of $\theta_{23}$

4. $P_{3}$ and $P_{4}$ are dependent on $\mathrm{CP}$ violating phase. $P_{3}$ is negative for neutrinos and positive for anti-neutrinos. So for experiments capable of observing oscillations in neutrino and anti-neutrino mode, the difference in $P\left(\nu_{\mu} \rightarrow \nu_{e}\right)$ and $P\left(\bar{\nu}_{\mu} \rightarrow \bar{\nu}_{e}\right)$ is $2 \times P_{3}$.

These measurements are made more complicated by the fact that over such long terrestrial baselines, neutrinos propagate mostly through earth's crust and the oscillation probabilities are altered by the matter effect. Consider equation 1.20. To gain some insight into the impact of interaction with matter on $\nu_{\mu}$ to $\nu_{e}$ oscillations, we make some approximations. Since $\theta_{13}$ is small, $\cos 2 \theta_{13} \sim 1$, and the denominator can be expanded to give

$$
\sin ^{2} 2 \theta_{13, \text { mat }} \sim \sin ^{2} 2 \theta_{13}\left(1 \stackrel{(-)}{+} \frac{4 \sqrt{2} G_{F} N_{e} E}{\Delta m_{31}^{2}}\right)
$$

where the minus sign is for anti-neutrinos. The $\sin ^{2} 2 \theta_{13}$ term defines the amplitude of the leading term in the oscillation probability, so the probability also changes proportionally to this factor in matter. Three important feature of this correction are:

1. The correction is inversely proportional to $\Delta m_{31}^{2}$, and therefore is sensitive to the neutrino mass hierarchy. 
2. For normal hierarchy $\Delta m_{31}^{2}$ is positive, and the matter effect leads to an enhancement in $\nu_{\mu}$ to $\nu_{e}$ oscillation probability and to a suppression in $\bar{\nu}_{\mu}$ to $\bar{\nu}_{e}$ oscillation probability. The effect is just the opposite in case of inverted hierarchy.

3. The matter effect increases linearly with energy. Because neutrino oscillations are a function of $L / E$, this implies that the longer the baseline of an experiment, more the effect of matter interactions on the oscillation probability.

A more accurate expansion of $\nu_{\mu}$ to $\nu_{e}$ oscillation probability is given in [23], and amounts to:

$$
\begin{aligned}
P\left(\stackrel{(-)}{\nu}_{\mu} \rightarrow \stackrel{(-)}{\nu}_{e}\right) \sim & \sin ^{2} 2 \theta_{13} \sin ^{2} \theta_{23} \frac{\sin ^{2}((1-A) \Delta)}{(1-A)^{2}} \\
& +\alpha \sin 2 \theta_{13} \cos \theta_{13} \sin 2 \theta_{12} \sin 2 \theta_{23} \frac{\sin (A \Delta)}{A} \\
& \frac{\sin ((1-A) \Delta)}{(1-A)}[\cos \delta \cos \Delta-\sin \delta \sin \Delta] \\
& +\alpha^{2} \cos \theta_{23}^{2} \sin ^{2} 2 \theta_{12} \frac{\sin ^{2}(A \Delta)}{A^{2}}
\end{aligned}
$$

where $A= \pm 2 \sqrt{2} G_{F} N_{e} E / \Delta m_{31}^{2}, \alpha=\Delta m_{21}^{2} / \Delta m_{31}^{2}$ and $\Delta=\Delta m_{31}^{2} L / 4 E$

The salient features of this more complicated form are consistent with those described above for the simplification. It is worth pointing out that due to the presence of matter effects, any difference between neutrino and anti-neutrino oscillation probabilities can not be interpreted crisply as a violation of CP symmetry, since the matter effects can alone produce such a difference. Therefore, all the interpretations of the parameters extracted from this mode are as a function of the $\mathrm{CP}$ violation phase, $\delta$ and the mass hierarchy.

The degeneracy between these parameters can be helped somewhat by making this measurement at different baselines, since that would change the contribution due to matter effects. 


\subsubsection{Experimental Observation}

There are three current experiments capable of making this measurement: $\mathrm{NO} \nu \mathrm{A}$, which is the subject of this dissertation, T2K and MINOS.

The MINOS detectors are made of alternating planes of steel and solid scintillator and the neutrino baseline is about $735 \mathrm{~km}$. The experiment was optimized for the $\nu_{\mu}$ disappearance measurement and is not very sensitive to $\nu_{e}$ appearance. But with sophisticated analysis techniques, they produced their first $\nu_{e}$ appearance result in 2013 and a combined $\nu_{\mu}$ disappearance and $\nu_{e}$ appearance result that mildly favors $\delta=3 \pi / 2$. [24].

The T2K experiment uses the Super-K water Cherenkov detector to detect accelerator neutrinos from the J-PARC accelerator complex. The neutrino energy peak is $0.6 \mathrm{GeV}$ and the baseline is $295 \mathrm{~km}$ long. Because of the shorter baseline, the effect of interactions with matter is appreciably smaller for T2K. The experiment has been collecting data since 2010. Their most recent result was published in April 2014, with the observation of $28 \nu_{e}$ candidate events and disfavors $0.35 \pi(0.09 \pi)<\delta<0.63 \pi(0.9 \pi)$ at $90 \% \mathrm{CL}$ for normal (inverted) mass hierarchy.

The $\mathrm{NO} \nu \mathrm{A}$ experiment is discussed in detail in the remaining length of this document. 


\section{Chapter 3}

\section{The $\mathrm{NO} \nu \mathrm{A}$ Experiment}

$\mathrm{NO} \nu \mathrm{A}$ is the NuMI Off-axis $\nu_{e}$ Appearance experiment, designed to measure oscillations of muon neutrinos in the NuMI beam at Fermilab. The power of the NO $\nu \mathrm{A}$ experimental setup comes from its location, capabilities of the NuMI beam and the $\mathrm{NO} \nu \mathrm{A}$ detector design. The location of the far detector is at a distance of $810 \mathrm{~km}$ from the neutrino source, near International Falls in Northern Minnesota, which enhances the matter effect (recall that the matter effect is more pronounced at higher energies, and therefore, at longer baselines). Figure 3.1 shows the $\nu_{\mu}$ to $\nu_{e}$ oscillation probability at a baseline of $810 \mathrm{~km}$, and its dependence, through matter effect, on the neutrino mass hierarchy. The oscillation probability peaks around $2 \mathrm{GeV}$ neutrino energy. To match the neutrino beam's energy peak to the $\nu_{\mu}$ to $\nu_{e}$ oscillation probability, the $\mathrm{NO} \nu \mathrm{A}$ detectors are placed 14 milliradians off-axis from the NuMI beam (see section 3.1).

NuMI can run in neutrino as well as anti-neutrino mode which enables measurements of $P\left(\nu_{\mu} \rightarrow \nu_{e}\right)$ and $P\left(\bar{\nu}_{\mu} \rightarrow \bar{\nu}_{e}\right)$. Figures 3.2 shows $P\left(\nu_{\mu} \rightarrow \nu_{e}\right)$ versus $P\left(\bar{\nu}_{\mu} \rightarrow \bar{\nu}_{e}\right)$ for varying $\mathrm{CP}$ violation phase, mass hierarchy and values of $\theta_{23}$ at $2 \mathrm{GeV}$ energy and a baseline of $810 \mathrm{~km}$. To first order, $\mathrm{NO} \nu \mathrm{A}$ will measure a point in this plane. The knowledge of parameters from other oscillation experiments constrains the range of possible observations by $\mathrm{NO} \nu \mathrm{A}$ to a set of ellipses. Due to the significantly large matter 

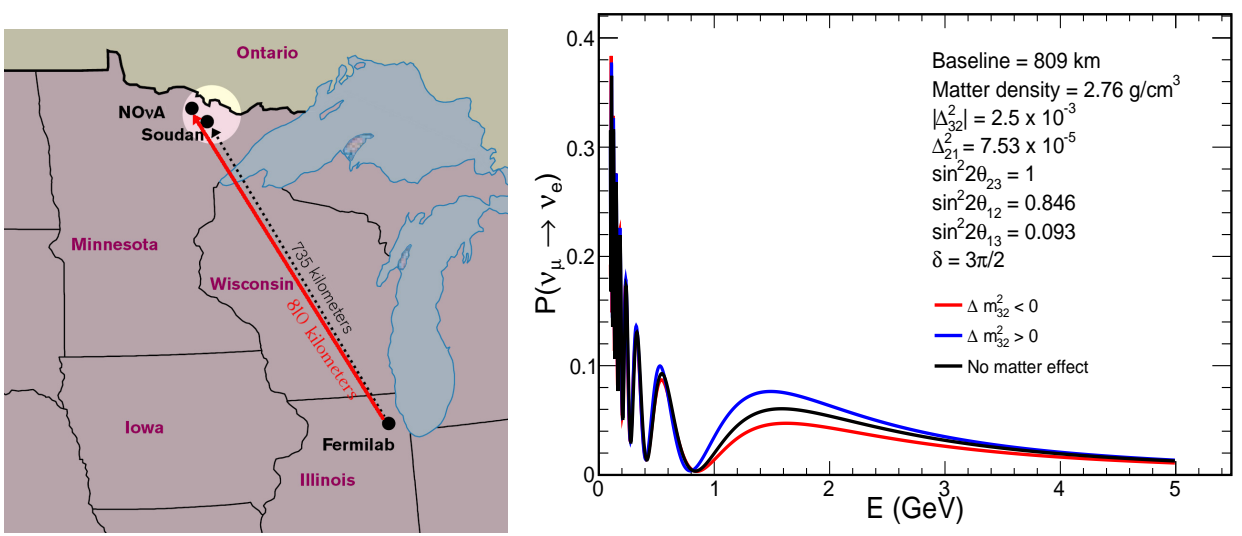

Figure 3.1: Location of the $\mathrm{NO} \nu \mathrm{A}$ detectors. The dotted black line indicates the axis of the NuMI beam, while the red line is 14 mrad off-axis from the beam. The plot shows the impact of matter effect on the $\nu_{e}$ appearance probability at $\mathrm{NO} \nu \mathrm{A}$ 's baseline.

effect, the ellipses are disparate for normal and inverted hierarchies. The amplitude of appearance probabilities is modulated by $\sin ^{2} \theta_{23}$. If $\sin ^{2} 2 \theta_{23}$ is different from 1 , the set of ellipses splits in two, one for $\theta_{23}>\pi / 4$ and the other for $\theta_{23}<\pi / 4$. The farther apart the point that $\mathrm{NO} \nu \mathrm{A}$ measures is from the alternative hypotheses, the tighter the constraints from the measurements.

The $\mathrm{NO} \nu \mathrm{A}$ detectors are optimized for $\nu_{e}$ identification. The $\nu_{e}$ appearance signal is charged current interactions of the electron neutrino which produces an electron in the final state. $\mathrm{NO} \nu \mathrm{A}$ detectors have been designed with low $\mathrm{Z}$ materials. This allows electromagnetic showers due to electrons to develop over many planes and cells and provide enough information for their identification.

$\mathrm{NO} \nu \mathrm{A}$ consists of two functionally identical detectors: a $0.3 \mathrm{kT}$ near detector (ND) about $1 \mathrm{~km}$ from the neutrino source at Fermilab and a $14 \mathrm{kT}$ far detector (FD) situated near International Falls, Minnesota. The purpose of the near detector is to measure the neutrino beam composition and energy spectrum close to the source, before neutrino oscillations set in. The far detector observes an oscillated neutrino beam and by 

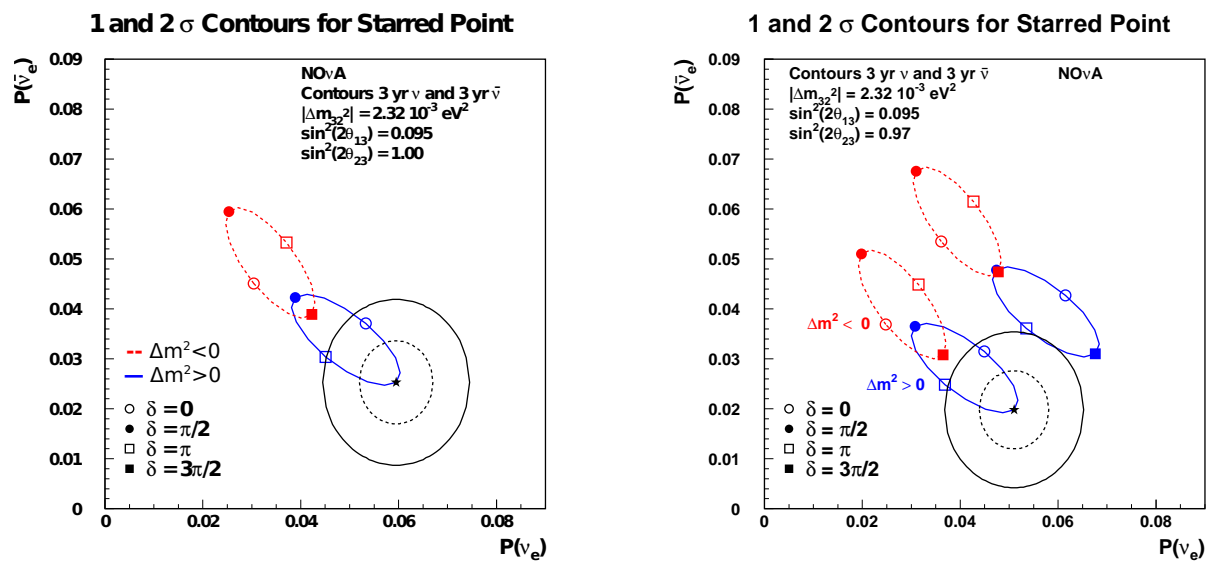

Figure 3.2: $P\left(\nu_{\mu} \rightarrow \nu_{e}\right)$ versus $P\left(\bar{\nu}_{\mu} \rightarrow \bar{\nu}_{e}\right)$ as a function of $\delta$ for the normal and inverted hierarchies. The star point is a possible measurement scenario that $\mathrm{NO} \nu \mathrm{A}$ may observe. The contours are representative statistical errors on the measurement.

comparing the beam composition and spectrum between the two detectors, oscillation parameters can be extracted. The near detector is $100 \mathrm{~m}$ below the surface in a cavern at Fermilab, and the far detector is on surface.

\subsection{The NuMI Beam}

The NuMI beam is a muon neutrino source operated by Fermilab in Batavia, Illinois. The neutrinos are produced by colliding high energy protons on a fixed target. The collisions produce a large number of charged pions and kaons. These hadrons are focused forward into a decay pipe, where their decays produce muon neutrinos: $\pi^{+} \rightarrow \mu^{+}+\nu_{\mu}$ (branching ratio $99.98 \%$ ) and $K^{+} \rightarrow \mu^{+}+\nu_{\mu}$ (branching ratio 63.55\%). The beam can be converted into an anti-muon neutrino beam by changing the polarity of the 
focusing horns and focusing negatively charged hadrons towards the decay pipe. The neutrino mode of the beam is called Forward Horn Current mode (FHC) and the antineutrino mode is called Reverse Horn Current mode (RHC). The spectrum of neutrinos obtained in this manner is reconstructed from $K / \pi$ production data, decay kinematics and simulation of the target-hall elements.
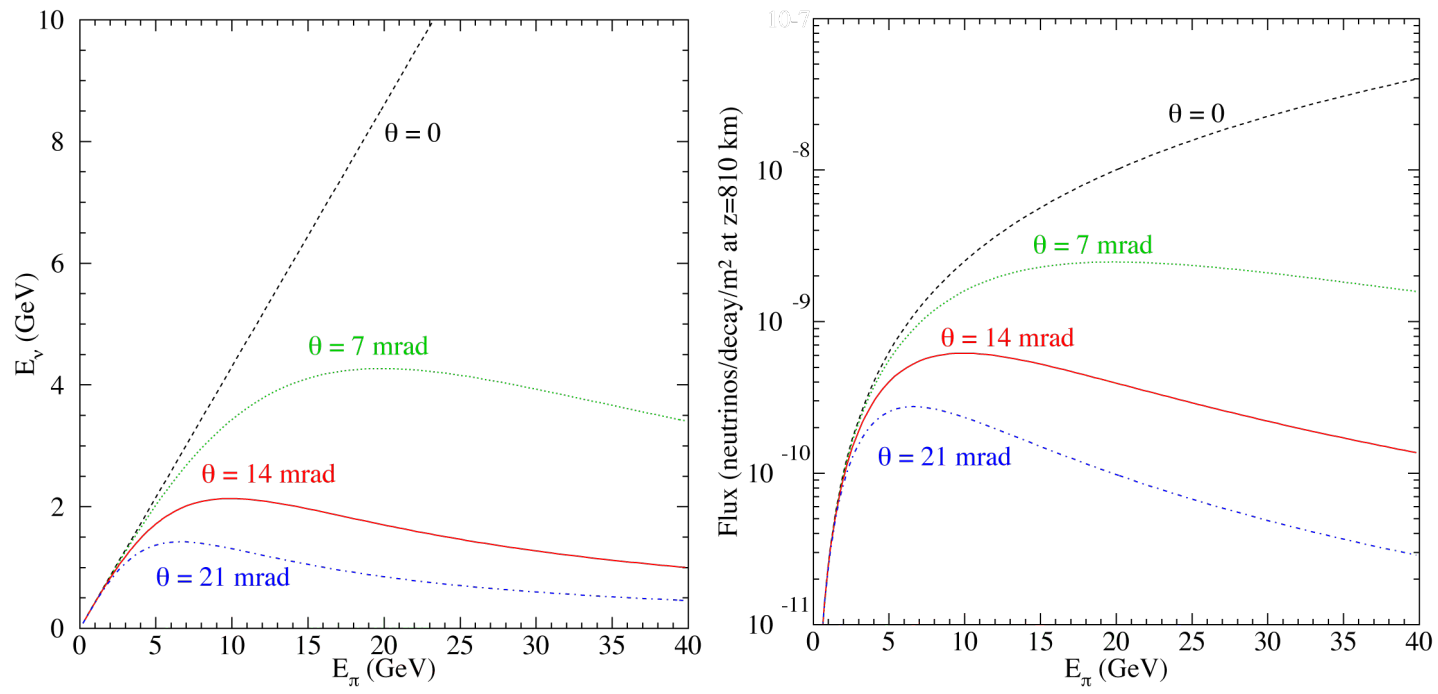

Figure 3.3: The neutrino energy spectra and flux as a function of the pion energy for different off-axis angles, $\theta$.

Since pions and kaons are spinless, their decay in the rest frame is isotropic. Using relativistic kinematics, it can be shown that the neutrino energy and flux as a function of the angle with respect to the beam axis in the lab-frame is given by

$$
\begin{aligned}
E_{\nu} & =\frac{a E_{h}}{1+\theta^{2} \gamma^{2}} \\
F & =\left(\frac{2 \gamma}{1+\theta^{2} \gamma^{2}}\right)^{2} \frac{A}{4 \pi L^{2}}
\end{aligned}
$$

where $h$ denotes the decaying hadron, and $a=\left(1-\frac{m_{\mu}^{2}}{m_{h}^{2}}\right) . \gamma$ is the hadron's boost, $A$ is the detector cross-section and $\theta$ is the angle between the hadron's and the decay neutrino's directions. It is clear from figure 3.3 that placing the $\mathrm{NO} \nu \mathrm{A}$ detectors 14 
milliradians off-axis from the beam ensures a narrow band beam with the neutrino energy peaked at about $2 \mathrm{GeV}$, which is close to the $\nu_{\mu}$ to $\nu_{e}$ oscillation probability maximum for a baseline of $810 \mathrm{~km}$. The narrowness of the spectrum reduces feed-down from the neutral current background.

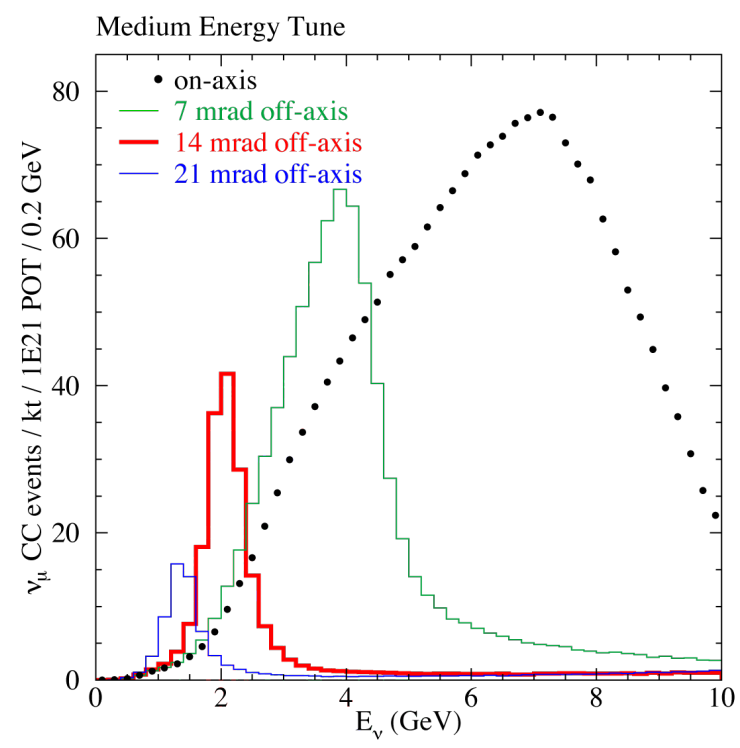

Figure 3.4: Simulated neutrino energy spectra at the $\mathrm{NO} \nu \mathrm{A}$ far detector baseline of 810 $\mathrm{km}$. The off-axis location of $\mathrm{NO} \nu \mathrm{A}$ suppress the high neutrino energy tail

The beam does have an intrinsic $\nu_{e}$ component resulting from $\mu^{+} \rightarrow e^{+}+\nu_{e}+\bar{\nu}_{\mu}$ and $K^{+} \rightarrow \pi^{0}+e^{+}+\nu_{e}$ (branching ratio $5.07 \%$ ). Since these decays are three-body decays, the energy spectrum of the resulting $\nu_{e}$ is much broader than the very narrow muon neutrino spectrum due to the off-axis location of $\mathrm{NO} \nu \mathrm{A}$. The electron neutrinos resulting from kaon decays are higher in energy, so the $\nu_{e}$ beam background for $\mathrm{NO} \nu \mathrm{A}$ is mostly due to muons decaying in the decay pipe. In the energy region of interest to $\mathrm{NO} \nu \mathrm{A}(1-3 \mathrm{GeV})$, our simulations show that the $\nu_{e}$ contamination is sub- $1 \%$ level. The beam has a small wrong-sign contamination too, ie anti-muon neutrinos in the neutrino beam and vice versa. The estimate for these components from simulation is $<2 \%$ in neutrino mode and $\sim 10 \%$ in the anti-neutrino mode. 


\subsubsection{The Accelerator Complex}

The Fermilab accelerator complex consists of two main accelerator rings. The Booster ring is fed protons from a linear accelerator and accelerates protons to $8 \mathrm{GeV}$. In the original design of the NuMI beamline, the proton batches from the Booster were transferred to the Main Injector ring, where they were accelerated to $120 \mathrm{GeV}$ energy. The beam in this configuration was used by the MINOS experiment for $\nu_{\mu}$ disappearance measurements and delivered a power $\sim 350 \mathrm{~kW}$.

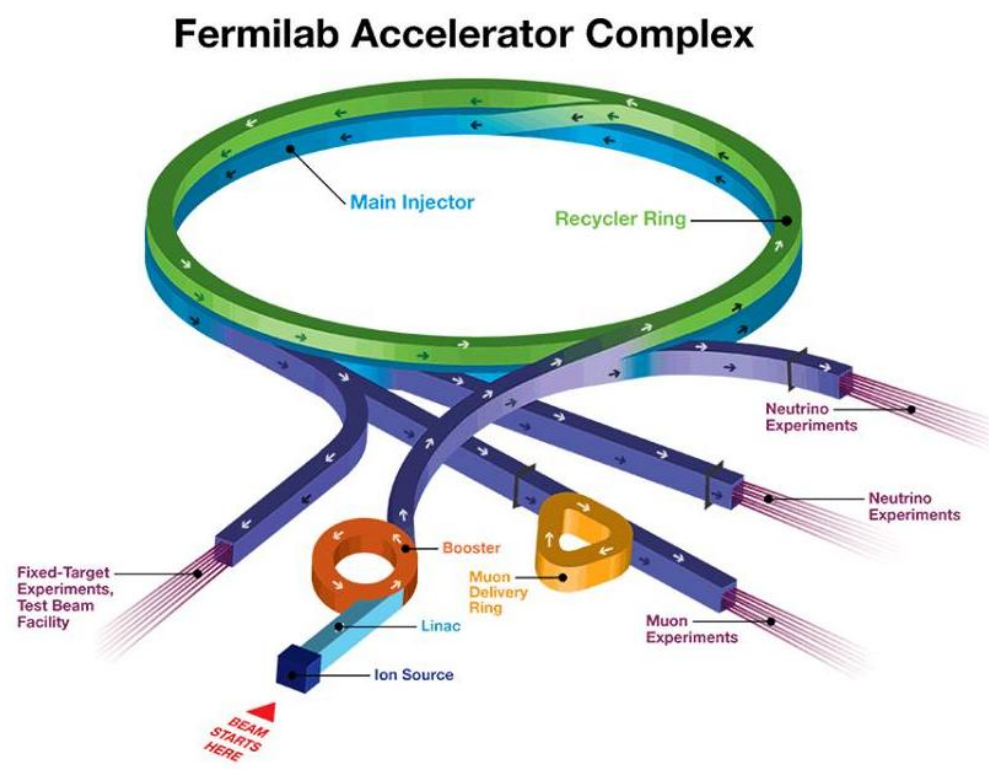

Figure 3.5: Schematic of the Fermilab accelerator complex, with upgrades implemented for $\mathrm{NO} \nu \mathrm{A}$

To increase the beam power for the $\mathrm{NO} \nu \mathrm{A}$ experiment, a series of upgrades have been implemented in the accelerator complex. The Main Injector cycle-time has been reduced from 2.2 seconds to 1.33 seconds. In the new scheme, the anti-proton storage ring from the Tevatron days has been converted into the Recycler ring. Protons from the Booster are now transferred first to the Recycler, which is used as a pre-injector to the Main Injector. This reduces the proton injection time from the Main Injector. 


\subsubsection{Slip-Stacking}

Another improvement is to slip-stack all the batches in the Recycler to improve the intensity of the NuMI beam. Fermilab uses two RF systems to implement slip-stacking. The details can be found in [25]; what follows is a brief qualitative description.

Six batches, with 4 to $5 \times 10^{12}$ protons each, are fed one after the other from the Booster into the Recycler. The Recycler is seven times larger than the Booster in circumference, which means that there is space in the Recycler for an extra batch. The first six batches in the Recycler are first decelerated, by gradually reducing the frequency of the RF cavity, and fall into a lower momentum orbit. A seventh batch is then introduced from the Booster into the Recycler, in the seventh, empty slot. Since the seventh batch is at a different momentum than the earlier six batches, the batches slip with respect to one another. The RF voltages are tuned such that the batches stay bunched, and before the next injection occurs, the seventh batch slips out of the seventh slot and lines up azimuthally with the sixth batch. The eighth batch is then introduced into the once again empty seventh slot. This process is repeated until all six batches have twice the number of protons, albeit at slightly different energies. The recycler beam is then extracted into the Main Injector altogether, so that the intensity of each Main Injector spill is close to $5 \times 10^{13}$ protons and it lasts for $10 \mu \mathrm{s}$.

\subsubsection{The NuMI Beamline}

The protons extracted from the main injector are directed $3^{\circ}$ downwards in order to aim the resulting neutrinos towards the MINOS far detector. The target for the $\mathrm{NO} \nu \mathrm{A}$ era is a series of graphite fins mounted between two stainless steel pipes that circulate water for cooling. The target is 2 pion interaction lengths, to provide sufficient probability of proton interaction, while avoiding absorption of low energy pions that are needed for neutrino production. The charged hadrons produced at the target are focused towards

the decay pipe by two pulsed magnetic horns whose inner conductors are parabolic 


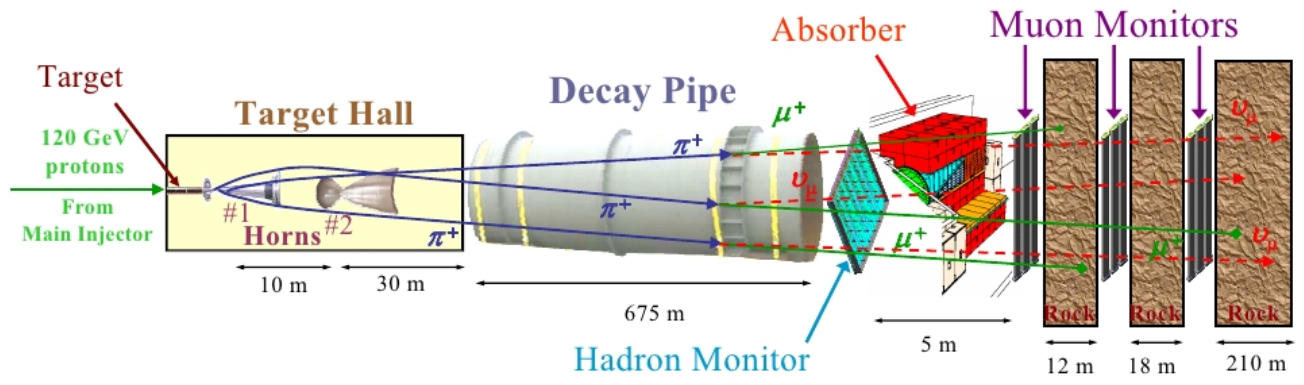

Figure 3.6: The NuMI Beamline [2]

in shape. The magnetic horns act like lenses with a focal length proportional to the pion momentum. The pions that are not focused by the first horn may be focused by the second horn downstream. The decay pipe is $675 \mathrm{~m}$ long and $1 \mathrm{~m}$ in radius and maintained at a very low pressure $\left(5 \times 10^{-5} \mathrm{~atm}\right)$. A water cooled Aluminium hadron absorber is placed at the end of the decay pipe, followed by three ionization chambers to monitor the outgoing muons.

\subsection{The NO $\nu$ A Detector Design}

The NO $\nu \mathrm{A}$ detectors are close to $65 \%$ active calorimeters, optimized for development of well-formed electromagnetic showers. Each of the detector components is described briefly in the following sections. This chapter draws heavily from the NO $\nu \mathrm{A}$ Technical Design Report [25] which may be consulted for greater detail.

\subsubsection{The $\mathrm{NO} \nu \mathrm{A}$ Unit Cell}

The NO $\nu \mathrm{A}$ detectors are composed of PVC modules extruded to form tube-like cells with rectangular cross-section $(\sim 4 \mathrm{~cm} \times 6 \mathrm{~cm})$. A layer of cells forms one plane of the detector. The cells in adjacent planes are orthogonallly rotated with respect to one another to allow for 3-D reconstruction of particle positions. Each cell is filled with 

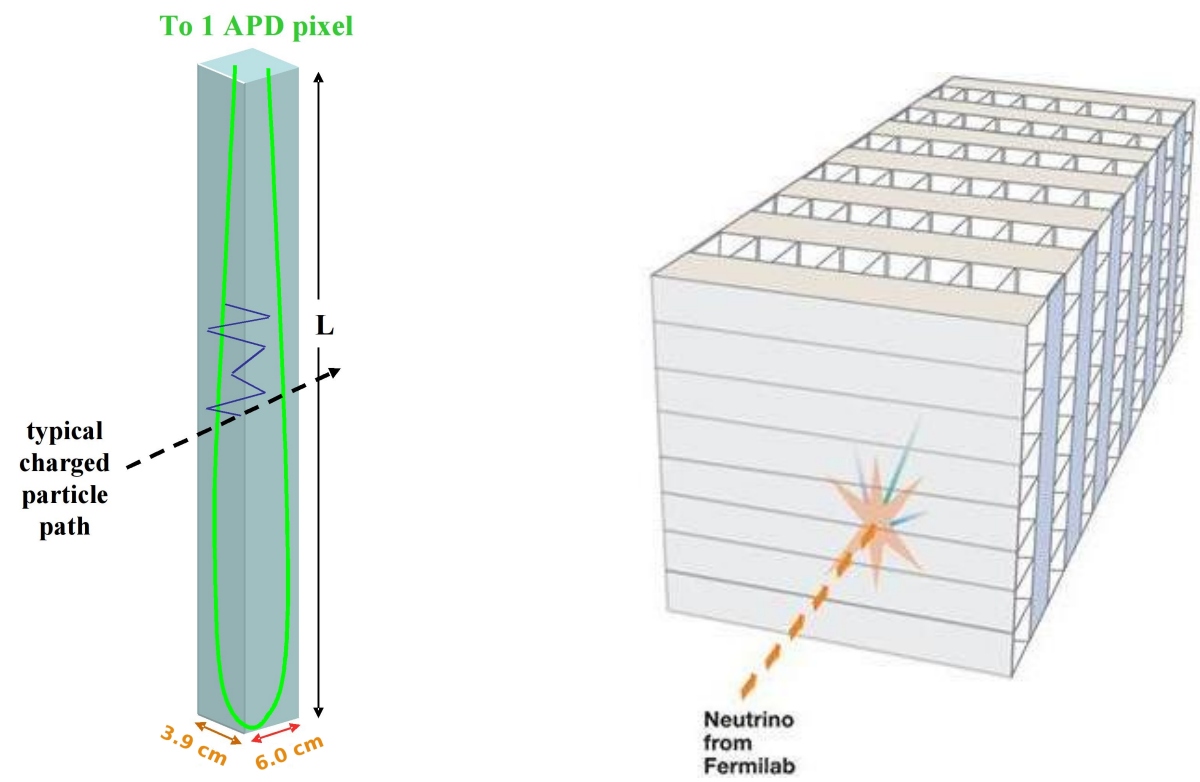

Figure 3.7: A cell and a cut-out of the $\mathrm{NO} \nu \mathrm{A}$ detectors indicating the orthogonal arrangment of cells in adjacent planes.

liquid scintillator and there is a loop of optical fiber to catch the scintillation light (see figure 3.7). The light from 32 such cells is read out by 32 pixels of an Avalanche Photo Diode chip. The cells are $3.6 \mathrm{~cm}$ wide and $5.6 \mathrm{~cm}$ deep. This defines the granularity of the $\mathrm{NO} \nu \mathrm{A}$ detectors. A cell is $15.5 \mathrm{~m}$ long in the FD and $4 \mathrm{~m}$ long in the ND. These long fiber lengths mean that light attenuation is a significant effect in $\mathrm{NO} \nu \mathrm{A}$. There are 384 cells per plane in the FD, and 48 in ND.

\subsubsection{Liquid Scintillator}

The scintillator used in $\mathrm{NO} \nu \mathrm{A}$ consists of three components: a scintillator that emits light in the UV region when charged particles pass through, wave-length shifters (WLS) that absorb the light emitted by the scintillator and emit longer wave-length radiation that can be picked up by the optical fiber and mineral oil as solvent, that blends these components into a stable solution. 


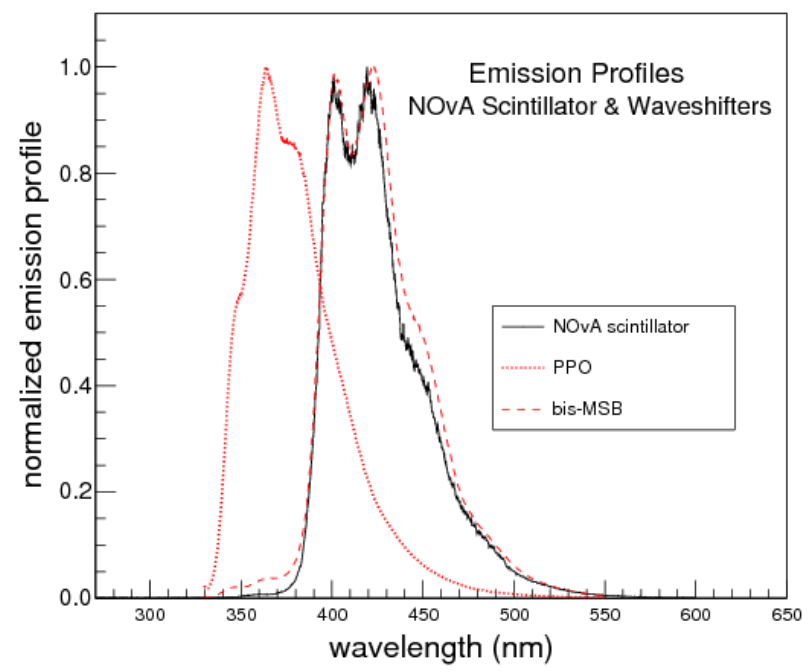

Figure 3.8: The emission spectrum of the $\mathrm{NO} \nu \mathrm{A}$ scintillator. The figure is from [3].

The scintillating agent used in $\mathrm{NO} \nu \mathrm{A}$ is pseudocumene $(1,2,4$ - Trimethylbenzene) that emits photons in the range $270-320 \mathrm{~nm}$. These photons emitted by pseudocumene excite the wave-length shifter PPO (2,5-Diphenyloxazole) that de-excites by emitting photons of wavelength in range $340-380 \mathrm{~nm}$. These photons excite the second wavelength shifter in the NO $\nu$ A blend, bis-MSB (1,4-bis-(o-methyl-styryl-)-benzene). This WLS de-excites through emission in the range 390 - $440 \mathrm{~nm}$ [3].

The $\mathrm{NO} \nu \mathrm{A}$ scintillator is close to $95 \%$ mineral oil and $5 \%$ pseudocumene, with sub-percent level of WLS agents. The scintillator also contains an anti-static agent (Stadis-425) at $3 \mathrm{ppm}$ to make it semi-conducting and less of a fire-hazard from charge build-up and sparking during filling. In addition, Vitamin $\mathrm{E}$ is added to the blend as an anti-oxidant to prevent yellowing of the scintillator, which would degrades its transparency over time.

$65 \%$ of the total mass of the $\mathrm{NO} \nu \mathrm{A}$ detectors is in liquid scintillator. 


\subsubsection{Optical Fiber}

The optical fiber used in the $\mathrm{NO} \nu \mathrm{A}$ detectors also contains wave-length shifting agents. The Y11 die in the fiber is excited by the violet light $(\sim 425 \mathrm{~nm})$ emitted by the $\mathrm{NO} \nu \mathrm{A}$ scintillator. The dye emits blue-green light in the wave-length range 450-650 nm. Due to the overlap between the absorption and emission spectrum of the dye, the wavelengths below $520 \mathrm{~nm}$ are severely attenuated in the fiber. The fiber core is

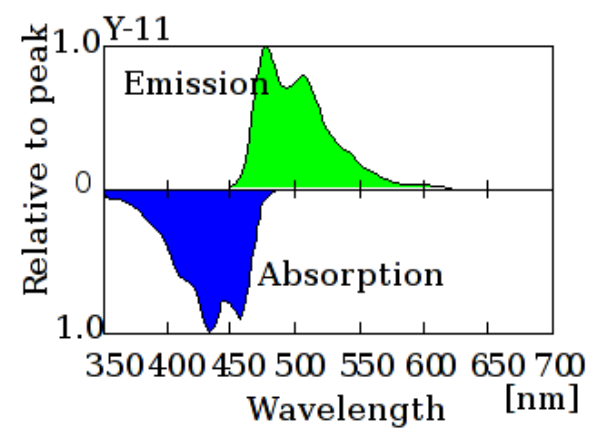

Figure 3.9: The absorption and emission spectra of the NO $\nu \mathrm{A}$ WLS fiber. Due to the overlap between the two, the light with wavelengths below $520 \mathrm{~nm}$ are highly attenuated.

made of polystyrene, which has a refractive index of 1.59 . The core is encased in an inner acrylic cladding $(\mathrm{n}=1.49)$ and an outer fluorinated-polymer cladding $(\mathrm{n}=1.42)$. The inner cladding improves the acceptance angle for total internal reflection of light in the core and also provides an additional layer of protection for the core. In the $\mathrm{NO} \nu \mathrm{A}$ cells, the fiber is placed in a loop, with its plane oriented along the diagonal of the cell cross-section. The bending radius of the fiber has to be as little as $3 \mathrm{~cm}$. For this reason the fiber has a radius of $7 \mathrm{~mm}$, which is much finer than the $1.2 \mathrm{~mm}$ fiber used in the MINOS detectors. The looped fiber provides twice the light-collection fraction than a single strand. It is also more efficient than two separate strands of fiber because it does not suffer from imperfect reflection from the fiber ends.

The two ends of the fiber in a single cell terminate at one pixel of a 32 pixel avalanche photo diode (APD) array. 


\subsubsection{Avalanche Photodiode (APD)}

APDs are photo-sensitive avalanche diodes that are operated with a high reverse bias voltage. Under such conditions, the electron and hole pairs excited by photons are accelerated in the strong internal electric field. As these highly energetic electrons move, they strike other electrons and cause them to be freed. These secondary electrons and holes are themselves accelerated and strike more electrons free and so on. This process is called impact-ionization and an avalanche of charge carriers in the diode leads to a significant amplification of the photocurrent.

APDs were favored over Photomultiplier Tubes (PMT) for light detection in $\mathrm{NO} \nu \mathrm{A}$ for two reasons: the quantum efficiency of APDs (85\%) is about eight times higher than that for PMTs $(\sim 10-20 \%)$ and is much flatter over the range of wavelengths of light that NO $\nu$ A WLS fiber transmits $(500-550 \mathrm{~nm})$. The high quantum efficiency of APDs is a necessity for $\mathrm{NO} \nu \mathrm{A}$ because it allows the observation of faint light signal from the end of $15 \mathrm{~m}$ long modules in the FD. The APDs used by $\mathrm{NO} \nu \mathrm{A}$ are manufactured by Hamamtsu and have a custom design to accommodate two ends of the fiber loop per cell on a single pixel. Each $\mathrm{NO} \nu \mathrm{A}$ APD array consists of 32 pixels, where each pixel reads out a single cell.

For APDs to effectively detect signal from the far end in a $15 \mathrm{~m}$ long cell, the noise must be kept low. To minimize the dark current (current in absence of light) due to thermally created electron-hole pairs, APDs are operated at a temperature of $-15^{\circ} \mathrm{C}$. Each APD carries its own thermoelectric cooler (TEC) to maintain this operational temperature. To remove heat from the TECs, a water cooling system has been designed which circulates water chilled to $15^{\circ} \mathrm{C}$. The APD surface must be kept clean and dry for safe operation and to minimize surface currents. To achieve this, the surface of the APD is coated with Parylene, a transparent epoxy, and it is ventilated with dry-air to prevent accumulation of moisture. 
In addition, the statistical nature of the avalanche process generates current fluctuations and the APD performance is further degraded by what is known as an excess noise factor. The excess noise is a function of the gain and the carrier ionization ratio, $k$, defined as the ratio of ionization probabilities of holes to electrons, which in the $\mathrm{NO} \nu \mathrm{A}$ APDs is about 1:50. The APDs are operated at a voltage close to $425 \mathrm{~V}$ which is known to produce a gain of about a 100. The excess noise factor is well-modeled and is included to some extent in our simulation. The operational conditions have been designed and demonstrated to produce a signal to noise ratio of 10:1 or better for majority of the APDs.

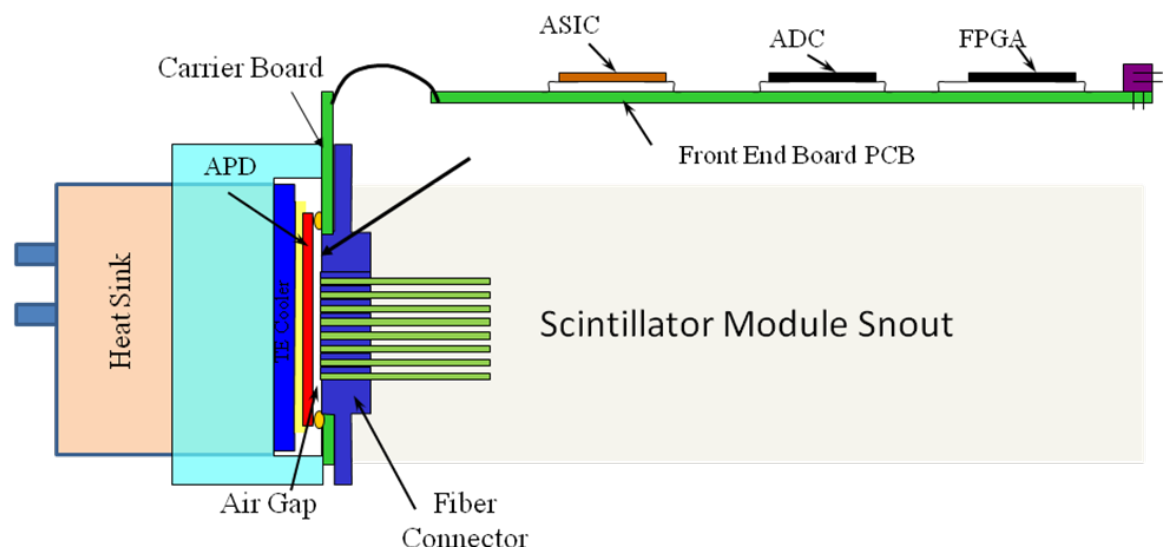

Figure 3.10: Schematic of the APD and front-end electronics.

The signal received from each APD pixel is further amplified through individual integrated circuits custom designed for $\mathrm{NO} \nu \mathrm{A}$. After amplification and pulse-shaping, the signal from eight cells is multiplexed into an ADC. The APD, and all the other components described here are mounted on a front-end board or FEB (see figure 3.10).

The APDs are operated continuously and do not require any external triggering.

\subsubsection{PVC Modules}

The PVC modules are the structural elements of the NO $\nu \mathrm{A}$ detector and primary containment for the liquid scintillator. $\mathrm{PVC}$ is close to $30 \%$ of the total $\mathrm{NO} \nu \mathrm{A}$ detector 
mass. The high light-yield of $\mathrm{NO} \nu \mathrm{A}$ is in part due to the reflectivity of the PVC modules, which is boosted substantially by adding anatase titanium dioxide $\left(\mathrm{TiO}_{2}\right)$ to it. The reflectivity attained in this manner is about $90 \%$ at the peak wavelength of scintillator emission (430 nm).

The shape of the PVC extrusions is optimized for easy and reliable extrusion, minimum PVC stress and light reflectivity. The outer extrusion walls are $4.8 \mathrm{~mm}$ thick and the inner walls between cells are $3.3 \mathrm{~mm}$ thick. The outer wall of the modules is scalloped at the cell boundaries due to the requirements of the extrusion process (see figure 3.11). The corners of the cells are rounded or scalloped too. This reduce PVC stress concentration on corners and makes the cell wall thickness constant which assists in uniform cooling in the extrusion process. The ratio of the inner to outer wall thickness is at the recommendation of the extruders for reliable extrusion.

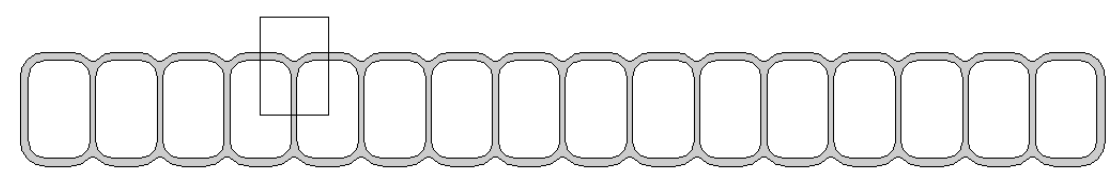

Figure 3.11: Profile of the PVC extrusion showing scalloped corners in the inner and outer walls of the extrusion

The PVC extrusions were extruded in groups of 16 cells and shipped, upon inspection, to the Module Factory at University of Minnesota, Minneapolis, where the extrusions and other components were assembled into $\mathrm{NO} \nu \mathrm{A}$ modules. This is done due to the expense and technical challenges involved in extruding 32 cell extrusions. For the assembly of the modules, adhesive epoxies that are inert to the liquid scintillator were used. The first step in the process is to glue two extrusions to form 32 cell extrusions. The cells are strung with loops of optical fiber. The end of the loop is mounted on a plastic ring, with a groove to hold the fiber in place, that is aligned along the diagonal of the cell's cross-section. At the top end of the modules, the two fiber ends from each of the 32 cells are embedded into the grooves of the fiber raceways. The 
raceway routes the fiber ends to the optical connector that contains 32 holes through which the fiber ends from the cells in the module are threaded. Each of the holes on the connector maps to a single channel on the APD. The handling of the fiber is a delicate process and tests were done to check the quality of the fiber at each stage of the process.

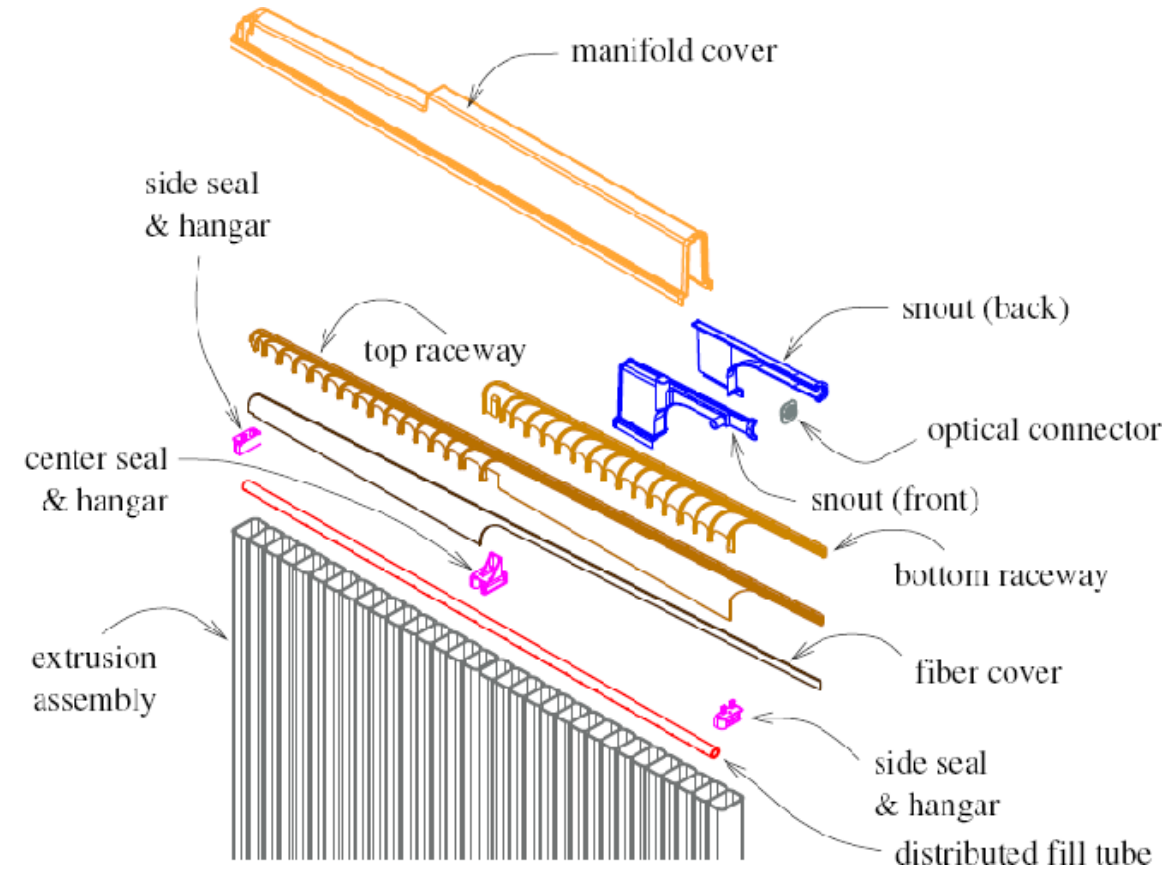

Figure 3.12: Schematic of the APD and front-end electronics.

The end of the module is sealed with an end plate, which is injection-molded from the same PVC as the extrusions. It has walls that cover the outside edge of the extrusions. Side seals are designed to take up the tolerance in the width of the module. End plate, center and side seals together seal the end of the module.

The top of the module is sealed with the manifold cover and the snout. The snout houses the optical connector and the fiber raceway ends and supports the APD and FEB mounted on the optical connectors. It also contains a port for filling the module with scintillator and another for ventilation. The manifold cover has a more complex shape than the end plate in order to accommodate the raceways. The shape of the manifold 
is with a step at the top to accommodate the snout from the adjacent module in the same plane. The fiber cover, raceways, manifold cover and snouts are all composed of black injection plastic to minimize light reflection. This reduces cross-talk between fibers from different cells in the module.

Once the modules are fully assembled, they are tested to detect any leaks. If a leak is found and the attempts to fix it prove unfruitful, the module is discarded. The fully tested modules are shipped to the far detector hall or to Fermilab to assemble the detectors.

\subsubsection{Detector Assembly}

\section{Far Detector}

In the far detector, each plane consists of 12 32-cell modules. The detector is assembled in units of blocks, each consisting of 32 planes glued together in alternating vertical and horizontal orientations. The fully assembled detector consists of 28 such blocks and has 896 planes in total. The south-end of the far detector hall is the assembly area where modules that are shipped from the module factory are stacked, reassessed for damage during shipping and prepared for assembly. Empty modules are vacuum lifted using an overhead crane. An automatic glue dispenser applies and spreads the glue on the surface of the modules. The modules are then moved into place on the block for adhesion. The bottom of the blocks is fitted with stiff steel pellets that provide structural support.

The assembly of the blocks takes place on a movable horizontal platform, known as the block pivoter. Upon completion of the block, the block pivoter moves on rails down the hall to the position where the block is to be installed. The horizontal platform pivots to a vertical position, installs the block in place, returns in a horizontal position to the assembly area, where the assembly of the next block commences. 


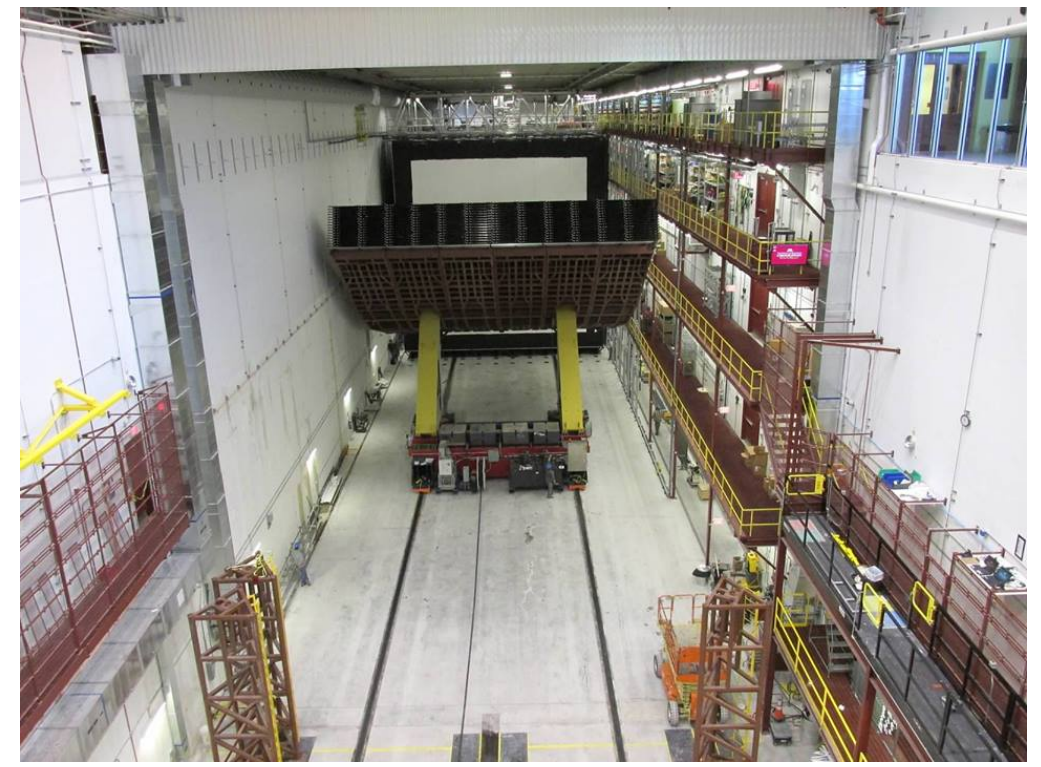

Figure 3.13: Block pivoter during the installation of a block in the far detector hall

The first block is installed against a concrete bookend that is capable of supporting the detector in case of buckling due to structural failures in the detector blocks. The subsequent blocks are installed against the already installed blocks. After the last block is installed, the block pivoter is pivoted against it and permanently left in that position, to provide support.

The blocks are filled with scintillator one at a time, starting first with the lowest horizontal modules. Once the horizontal modules are filled, the filling of vertical modules follows. Once the block is filled, the modules are outfitted with APDs and FEBs.

\section{Near Detector}

The processes involved in the assembly of the near detector are the same as those for the far detector with a few modifications. The near detector has three modules per plane and 24 planes (12 horizontal and 12 vertical) per block and contains 8 such blocks in total. In addition, the downstream end of the ND has a muon catcher to range out muons. 
The muon catcher contains ten planes of steel, each of which is 4.03 inches in thickness, as wide as three module-widths and as tall as two module-widths, giving them a rectangular shape. The steel planes are interspersed with three vertical and two horizontal PVC planes for detecting muons. The vertical PVC modules are also $2 / 3$ their usual height to match the steel planes. Therefore, the total number of active, scintillator filled PVC planes in the ND is 214.

Once a near detector block is assembled, it is erected into an upright position. A cradle is then built around it for safe transportation to the underground detector cavern. The blocks arrive at the MINOS surface building at Fermilab and are lowered down the the MINOS shaft. The detector blocks are on wheels which are a part of the cradle. Once underground, they are wheeled into place in the $\mathrm{NO} \nu \mathrm{A}$ near detector hall.

\section{Summary}

The NO $\nu \mathrm{A}$ detectors are composed of liquid scintillator filled PVC modules. Wavelength shifting fiber and APD's are the light-detection elements in the detector design. The detectors are now fully constructed. The far detector has been taking data since October 2013 and the near detector, since August 2014. 


\section{Chapter 4}

\section{Data Acquisition and Triggering Systems}

The $\mathrm{NO} \nu \mathrm{A}$ detector design presents unique challenges in data acquisition. The far detector is located $810 \mathrm{~km}$ from the NuMI beamline so triggering the readout in advance of a NuMI beam spill is difficult. Using an activity based trigger to record NuMI data is also difficult because the FD is located on surface and there is a very large flux of cosmic rays, at $120 \mathrm{kHz}$. These problems are circumvented by operating the detector in continuous readout mode which is outlined in this chapter.

\subsection{Overview of NO $\nu \mathrm{A}$ DAQ System}

The pulses from 32 channels of an APD (Avalanche Photodiode) are readout by a Frontend Board (FEB) that time stamps and shapes the signal. Data from 64 FEBs (and therefore 64 modules, with 32 cells each) are aggregated by a Data Concentrator Module or DCM, which is a custom built computer. It organizes data into $50 \mu$ s long units, or microslices. These $50 \mu$ s units are aggregated by an event builder module, running on the DCM, into $5 \mathrm{~ms}$ long chunks, or millislices. The durations of these data aggregation units are optimized for transmission over the internal bus and on the ethernet. All the 
DCMs dispatch their $5 \mathrm{~ms}$ millislices to a pool of buffer nodes, such that the data from the same $5 \mathrm{~ms}$ window from all DCMs arrives at the same buffer node.

Two consecutive modules in 32 consecutive planes from the same view are read out by a single DCM. Since each block contains only 16 modules per view, a DCM encompasses two blocks, a unit we call diblock. Therefore, while the $\mathrm{NO} \nu \mathrm{A}$ detectors are installed in units of blocks, the readout is organized in units of diblocks. A diblock contains 64 planes, 32 in each view. It is instrumented with 12 DCMs, 6 for horizontal and 6 for vertical view (recall that there are 12 modules per plane). In figure 4.1, DCMs are outline in green. The mass of a single diblock is about $1 \mathrm{kT}$, and there are 14 of them in the completed $\mathrm{NO} \nu \mathrm{A}$ far detector.

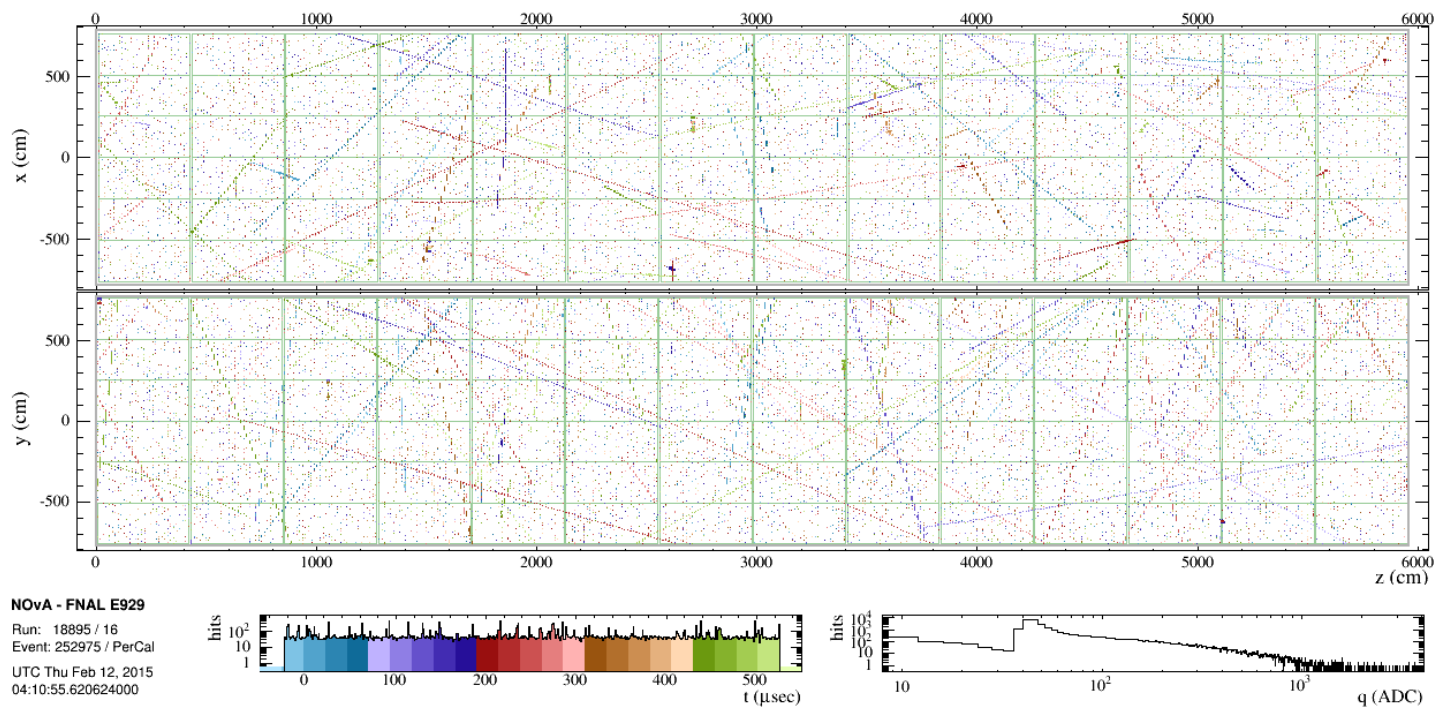

Figure 4.1: DCM layout in FD. The green boxes indicate the readout domain of an individual DCM.

\subsection{Clock and Triggering}

All the readout components in $\mathrm{NO} \nu \mathrm{A}$ are synchronized to an external wall clock through the timing chain at the head of which is a Master Timing Distribution Unit (MTDU). 
The MTDU is connected to a high-precision GPS receiver to drive the master clock. The MTDU drives slave TDUs (STDU), each of which synchronizes a diblock, or 1kT of the $\mathrm{NO} \nu \mathrm{A}$ detector. This results in all the detector channels being synchronized with each other. The detector is operated in a free running mode where all the channels that register an above-threshold energy deposit record a hit with a time stamp.

The MTDU at Fermilab is connected to the accelerator network. When a beam spill occurs at Fermilab, it decodes the accelerator time stamp into $\mathrm{NO} \nu \mathrm{A}$ time, and the spill is transmitted to both near and far detectors, after correcting for the time of flight of neutrinos through the earth. When the spill information is received by the spill receiver, the buffer nodes search in millislices for hits that occurred in the beam spill time and the selected hits are stored to permanent storage. Note that the far detector beam spills are recorded in $500 \mu$ s windows, centered around the beam spill time. This is because the exact location of the spills was not known with enough accuracy and has since been determined using the far detector data (see section 4.4.1). Moreover, recording beam-spills with ample padding on either side allows for cosmic background estimation from these side-bands. The buffer nodes are capable of storing up to 20 minutes of far detector data and the spill messages are received between 2-10 seconds after the occurrence of the spill.

Additionally, we also run a cosmic pulsar trigger at the far detector, that triggers at regular intervals and records $500 \mu s$ of cosmic data at a time. In the near detector, the cosmic trigger is an activity based trigger. The cosmic data is used for calibration, to determine timing resolution and study detector response and also, to study cosmic background to the neutrino oscillation analyses. More details on the $\mathrm{NO} \nu \mathrm{A}$ timing and triggering systems can be found in [26]. 


\subsection{Readout}

The front-end board (FEB) that an APD is mounted on houses an ASIC (Application Specific Integrated Circuit) to shape the signal and an ADC (Analog to Digital Converter) to digitize it. The far detector FEBs are driven by a $16 \mathrm{MHz}$ clock and the ASIC uses 8:1 multiplexing to readout the 32 channels of an APD. This means that each APD channel is readout with a $2 \mathrm{MHz}$ frequency. The far detector ASIC shapes the pulse with a $460 \mathrm{~ns}$ rise-time and a $7000 \mathrm{~ns}$ fall-time. These shaping parameters are determined from measuring the APD leakage current and shot noise to get a signal to noise ratio of 10:1 from the far end of the cell.

The near detector, due to its proximity to the neutrino beamline, records multiple interactions per beam spill. The separation of hits arising from different neutrino interactions requires a more precise timing resolution in the near detector than in the FD. The ND ASIC uses 2:1 multiplexing, so the sampling frequency of each APD channel is increased to $8 \mathrm{Mz}$. In addition, a pulse in the near detector is shaped with a rise-time of $140 \mathrm{~ns}$ and a fall time of $4500 \mathrm{~ns}$.

The FEBs use a dual correlated sampling (DCS) algorithm to determine if a pulse is signal or not. In this algorithm, the FEB processes the difference between the ADC counts of the $s_{i}$ sample and the ADC count from the sample three clock-ticks prior, $s_{i-3}$. If this difference is above a pre-determined threshold value, the baseline subtracted ADC value of the $s_{i}$ sample and its time, ie the TDC (Time to Digital Converter) value are recorded as a hit. This mode is known as the single-point mode. We can also store multiple samples, $s_{i-3}$ to $s_{i}$, which we may fit offline to more precisely estimate the hit time from the rising edge of the pulse. This readout mode is called the multi-point readout. The near detector has always been operated in the multi-point mode. The far detector was operated in single-point mode until October 2014, and in multi-point mode since. Figure 4.2 shows a cartoon of a typical pulse shape. In the single point readout mode, the timing resolution of the far and near detectors are $\left(t_{\text {sample }} / \sqrt{12}=\right)$ 
144 ns and 36 ns, respectively.
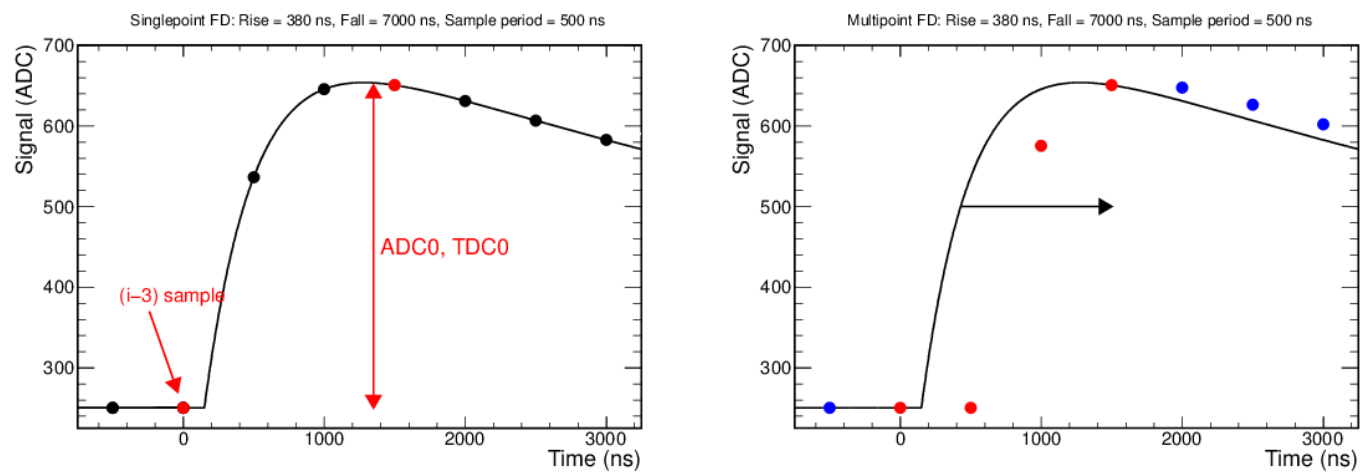

Figure 4.2: Single- and multi-point readout. In single-point readout, the charge and time of the baseline subtracted, above threshold $i$ th sample is saved, while in multipoint mode, a pulse-shape is fitted to the trace to derive the time and charge of the hit.

\subsubsection{APD Sag and Ringing}

Benchtop charge-injection tests of APDs have shown that when any particular channel on an APD is triggered, the other 31 channels exhibit a negative pulse, ie a drop in the output. The observed pulse on the triggered channel itself is diminished by the same magnitude as the drop in other channels. The scale of the drop is found to be linearly correlated with the amount of charge injected on a channel, and is about $1.86 \%$ of the injected charge. When multiple channels are triggered, the drop in the baseline across all channels is found to be $1.86 \%$ of the total charge deposited in the APD. The cause of this drop is not well understood. Simulated waveforms of channels triggered in a single APD in the ND are shown in figure 4.3. Notice the black trace of a channel that sags below zero due to the presence of charge on other channels.

A repercussion of APD baseline sag is APD ringing or flashing. When the baseline of untriggered channels recovers from sag by returning to the nominal baseline, if the 


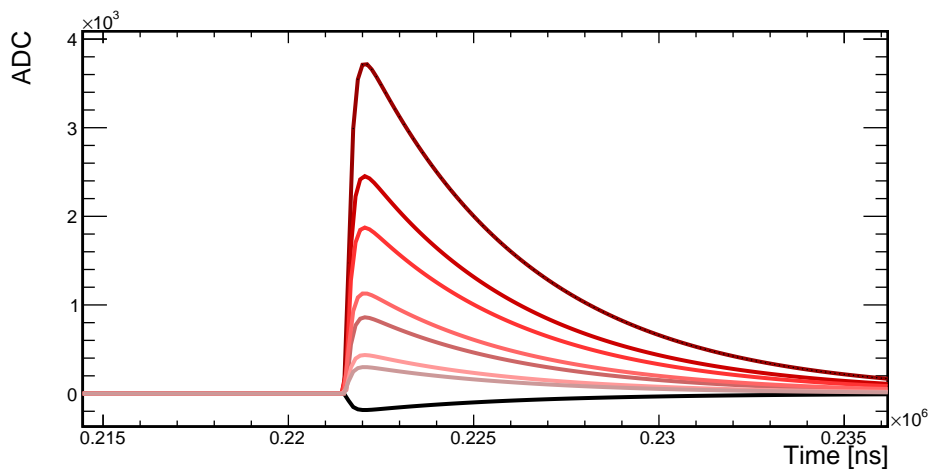

Figure 4.3: Simulated readout traces of channels on a single APD exhibiting sag. The black trace is of a channel that had no energy deposited in it, but the baseline sags due to triggering of other channels on the APD.

difference between any pairs of samples separated by three clock-ticks exceeds the ADC threshold of that channel, the Dual-Correlated Sampling (DCS) algorithm triggers a hit. Since the sag is larger when the the initial charge deposited on the APD is high, this flashing of an APD is observed after a very a highly ionizing interaction takes place. Bench top tests show sag induced triggering to occur with charge deposits of $250 \mathrm{X}$ the threshold in FD and 700X in ND. This translates to $\sim 300 \mathrm{MeV}$ and $\sim 500 \mathrm{MeV}$ of energy deposited in a far and near detector APD, respectively. APD flashes usually happen several microseconds after the initial charge deposition and therefore are well-separated from the main event in time.

\subsection{Timing Peak}

\subsubsection{Far Detector}

In the far detector, the data are dominated by cosmic rays. We apply a series of cuts to reject cosmic rays and select neutrino-like interactions. The timing distribution of selected events is used to determine the location of the NuMI peak. The cuts employed 
for this analysis are containment cuts and requiring the event to have low transverse momentum fraction with respect to the NuMI beam direction. Some of these concepts of neutrino event selection are discussed in detail in chapter 8. The time used is the meantime of all hits in a neutrino interaction (separation of hits due to unrelated interactions into distinct units is discussed in section 7.3).

The result of the timing peak search is shown in figure 4.4. The red lines indicate the spill boundaries. The horizontal blue line is a fit to background outside the spill time. The event count is absent from the y-axis for blinding purposes (see section 5.1.1 for a discussion on blinding). The NuMI neutrino peak is found between 218 and 228 $\mu$ s with a significance of $>12 \sigma$.

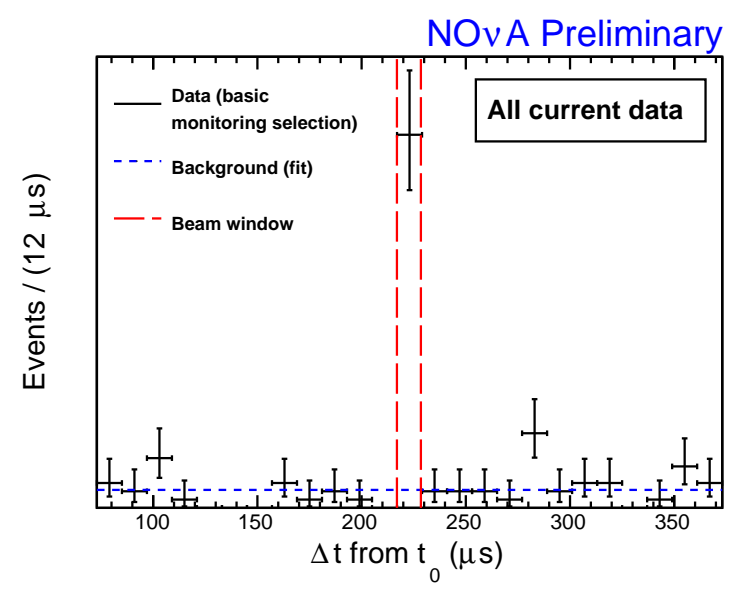

Figure 4.4: Far detector timing peak, over the full $500 \mu$ s window, with all data upto February 16, 2015.

\section{The Second Peak}

The Timing Distribution Units (TDUs) have a known but rare failure mode that when they are rebooted, their clocks can initialize with a $64 \mu$ s offset. If this occurs in a master TDU (MTDU), it causes a mismatch between the expected beam spill time and the actual time at which it occurred, until the next time it is rebooted. This error mode 
is easy to monitor for in the near detector, since the neutrino peak moves visibly. In the far detector, these cases were not identified at the time, but in retrospect, we see compelling evidence of this shift in the far detector for at least some part of the data collected thus far. This can be seen in the timing peak in figure 4.4, where a smaller peak $64 \mu$ s after the expected peak at $218 \mu \mathrm{s}$, at $282 \mu \mathrm{s}$ is visible.

The far detector was only susceptible to this failure before the October beam shutdown. During the shutdown, a Timing Calibration Reference (TCR) was installed at each detector. The TCRs have an independent GPS antenna and receivers that generate a stable $1 \mathrm{~Hz}$ pulse. MTDUs receive these signals and time stamp them. If the MTDU version of time is different from expected, an automated synchronization process is started.

The treatment of this additional timing window at $282 \mu \mathrm{s}$ and its consequences to the analysis of those data are discussed in the next chapter.

\subsubsection{Near Detector}

Observing the timing peak in the near detector is trivial in comparison to the far detector. It is $100 \mathrm{~m}$ underground, and the cosmic interaction rate in the detector is negligible. Therefore, the activity that is registered in the detector is mostly due to the interaction of neutrinos. Electronic noise is evenly distributed in time, while the neutrino interactions occur within the $10 \mu \mathrm{s}$ NuMI spill. The NuMI beam peak is discernible by just plotting the hit times within the $500 \mu$ s NuMI trigger window, with no cuts applied. The peak can be observed after collecting a few spills of NuMI data. The location of the spill in the $500 \mu$ s trigger window is found to be consistent with the expectation of $218-228 \mu \mathrm{s}$.

Because of the large statistics and reasonably precise timing, we are also able to observe (figure 4.5) the batch structure in NuMI spills (recall that the NuMI spill is composed of six batches of protons, see 3.1.2). 

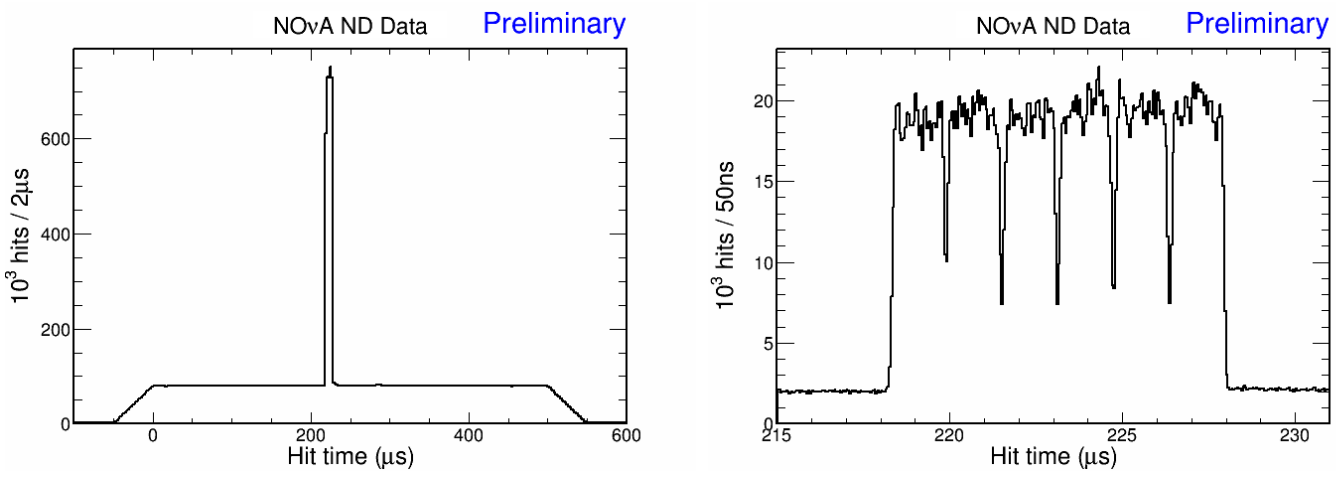

Figure 4.5: Near detector timing peak, over the full $500 \mu$ s window, and zoomed in to show the NuMI beam structure. 


\section{Chapter 5}

\section{$\nu_{e}$ Appearance Analysis Overview}

The analysis presented in this report is an observation of $\nu_{e}$ appearance in the muon neutrino NuMI beam in the $\mathrm{NO} \nu \mathrm{A}$ experiment. This chapter lays out the steps involved in this analysis and attempts to provide some context for the chapters that follow. Section 5.2 is dedicated to describing the data analyzed in this thesis.

\subsection{Oscillation Analysis Steps}

In a two-detector oscillation experiment such as $\mathrm{NO} \nu \mathrm{A}$, the neutrino data collected in the near detector are used as a control sample. Given that the neutrinos are 2 $\mathrm{GeV}$ in energy at the off-axis location of $\mathrm{NO} \nu \mathrm{A}$, the propagation distance of neutrinos from the source to the near detector $(\sim 1 \mathrm{~km})$ is much smaller than the oscillation length. Therefore the ND neutrino data contains no $\nu_{e}$ appearance signal and is a pure background-only sample. The use of the ND data and other steps and concepts used in the appearance analysis are described below.

\subsubsection{Blind Analysis}

The oscillation analyses in $\mathrm{NO} \nu \mathrm{A}$ are blind analyses, which means that all cuts, background and error estimates etc are finalized prior to looking at the far detector neutrino 
data. Blinding is achieved by obscuring relevant information about the events in the NuMI trigger data that relate to the oscillation probabilities.

The purpose of blinding is to avoid any bias in our oscillation results. The two pieces of information that are sufficient for the oscillation analysis are the flavor of the neutrino and the energy of the interaction. So the particle identification (PID) information and energy are naturally blinded. Other variables from which event energy or identity may be deduced, such as the leading shower energy or length, number of planes and cells in slice, PID input variables etc, are also blinded. Blinding is only applied to the far detector data events with reconstructed energy between 0.8 and $3 \mathrm{GeV}$, that fall within the $10 \mu$ s beam window in the NuMI trigger stream. Leaving beam events that do not bias our oscillation results unblinded allows the study of sidebands that offer valuable sanity checks of our reconstruction and PID on the far detector data. It is worth noting that the near detector data are not subject to blinding which lets us put our simulation to more severe tests against the neutrino data.

\subsubsection{Cosmic Background Estimate}

As described in the previous chapter, we have a dedicated cosmic trigger in the far detector, which records the data collected over a $500 \mu$ s window at regular intervals. The data from this trigger stream was used to tune the cosmic rejection cuts. The NuMI trigger windows are also $500 \mu \mathrm{s}$ in duration, while the beam only lasts for $10 \mu \mathrm{s}$, from 218 to $228 \mu \mathrm{s}$. NuMI trigger data outside of the time range $217-229 \mu$ s is called the timing sideband and is used to estimate the cosmic background for the final analysis. Using the NuMI trigger ensures that the detector configuration and data-taking conditions used in cosmic background estimates are identical to that of the neutrino data.

Figure 5.1 is a diagramatic representation of how a NuMI trigger is split. The NuMI spill times are padded by $1 \mu$ s so neutrino data is not lost due to slight miscalibration of our timing system. For cosmic background estimate, we get further away from the beam spill and use data in the two sidebands between 25 and $208 \mu$ s and 238 and 475 
$\mu \mathrm{s}$. Any cosmic events in the sidebands that pass the $\nu_{e}$ selection cuts are scaled by a factor of $12 /((208-25)+(475-238))=12 / 420$ to scale to the livetime of the NuMI beam spill for a final estimate of the cosmic background.

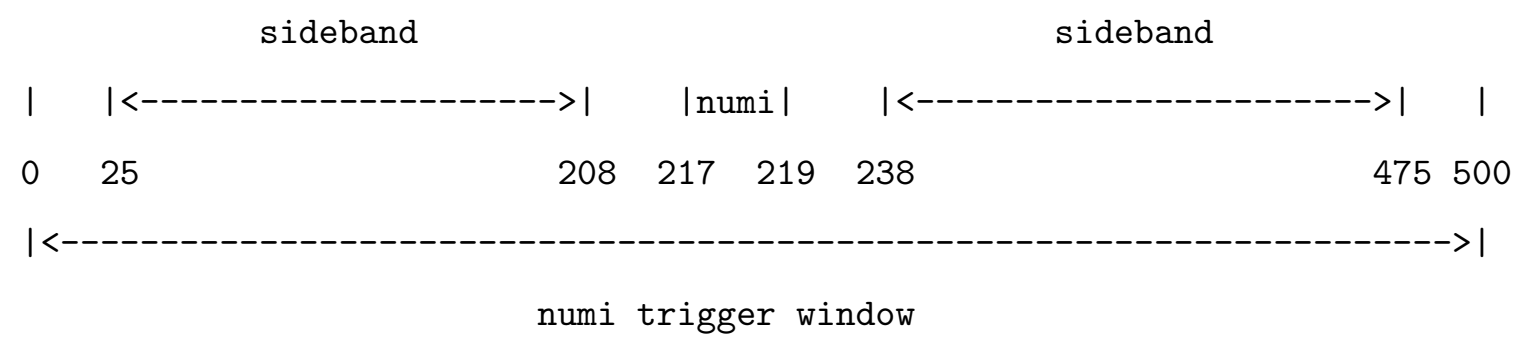

Figure 5.1: Diagram showing the timing sideband in NuMI trigger windows. The numbers are times in $\mu$ s since the start of the NuMI trigger window. The sidebands are used to estimate cosmic background.

\subsubsection{Beam Background Estimate}

Since there is no $\nu_{e}$ appearance signal in the near detector, any events that pass the $\nu_{e}$ selection cuts represent background to the $\nu_{e}$ appearance analysis. However, event counts alone are not sufficient. The background selected in the ND must be broken down by event type so that they may be properly treated in oscillation. For instance, neutral current interactions are flavor independent and therefore, unaffected by oscillations, while muon neutrinos oscillate, therefore the contribution of $\nu_{\mu} \mathrm{CC}$ events to background in FD will be greatly diminished to that in the ND.

The estimation of the contribution of different components to the observed background in the near detector is known as decomposition. This is discussed in more detail in chapter 11 


\subsubsection{Extrapolation}

The ND data is used not only for beam background estimates, but also for prediction of the $\nu_{e}$ appearance signal. The observed $\nu_{\mu} \mathrm{CC}$ interaction rate in the ND is essentially a measurement of the beam at $t, L=0$ and translates to an expectation of $\nu_{e}$ appearance signal in the FD. It gives us a handle on any beam flux differences in data and MC that would impact the $\nu_{e}$ appearance signal.

The conversion of the observed interaction rates in various channels in the near detector to a prediction of signal and background in the far detector is known as extrapolation. The extrapolation method is discussed in detail in chapter 11 .

\subsubsection{Final Fit}

The signal expected (see chapter 11) from the analysis of the first $\mathrm{NO} \nu \mathrm{A}$ data is small, so we have opted for a simple cut-and-count analysis. That is, our measurement of oscillation parameters is based solely on the number of events observed in the FD, and not their underlying energy distribution. Extrapolation of beam background and cosmic background estimate from the NuMI timing sidebands gives us an estimate of the event counts expected in the absence of muon to electron neutrino oscillation. Any excess observed over this total background estimate will be interpreted as an observation of $\nu_{e}$ appearance and the result will be reported as a significance of appearance and values of oscillation parameters that favor the observed event counts. The fitting framework, estimates of systematic errors and the final results are discussed in chapter 13 .

\subsection{NO $\nu$ A's First Data}

$\mathrm{NO} \nu \mathrm{A}$ 's first data, which are analyzed here, are the data taken from February 06, 2014 till May 15, 2015 in the far detector and from August 16, 2014 till March 13, 2015 in the near detector. Data taken prior to these periods were when the respective detector was being commissioned and therefore are not analyzed for an oscillation result. The near 
detector data span a shorter duration than the far detector data because it was built later than the far detector, and within a short period of time gathered enough statistics required for the analysis. The ND data period from March 13, 2015 to May 15, 2015 is not included because the data could not be processed in time for use in this analysis. In this analysis, we only consider FD data taken with 4 or more contiguous diblocks.

We define the far detector exposure as mass in kilotons times POT collected, to be able to compare the relative exposure in different diblock configurations. The first FD data are equivalent to $32.1 \times 10^{20} \mathrm{POT} \times \mathrm{kT}$. Livetime exposure is expressed in mass times seconds for the same reason and is based on the assumption of a $10 \mu$ s beam spill with $1 \mu$ s padding at each end.

The Fall 2014 NuMI beam shutdown (from September 01, 2014 to October 30, 2014) divides the first data in two parts, pre- and post-shutdown data. The shutdown was used as an opportunity to update and improve various detector systems. Timing Calibration References (TCR) were installed at the detectors and far detector readout was switched to multi-point mode.

\begin{tabular}{l|lll}
\hline & Pre-shutdown & Post-shutdown & Total \\
\hline POT $\left(\times 10^{20}\right)$ & 1.27 & 2.25 & 3.52 \\
Exposure $\left(\mathrm{POT} \times \mathrm{kT} \times 10^{20}\right)$ & 7.3 & 24.8 & 32.1 \\
Livetime $(\mathrm{s})$ & 131.85 & 102.23 & 234.09 \\
Livetime Exposure $(\mathrm{s} \times \mathrm{kT})$ & 759 & 1123 & 1882 \\
\hline
\end{tabular}

Table 5.1: Far detector exposure and livetime

\subsubsection{Treatment of $64 \mu$ s Delayed Peak in Pre-Shutdown Data}

As discussed in section 4.4.1, before the installation of the TCRs during the shutdown, the $64 \mu$ s offset in MTDUs went unnoticed, and there may have been several periods when the data were taken in this state. So for pre-shutdown data in FD, there is no 
way for us to know whether the beam spills occurred at the usual 218 to $228 \mu$ s from the start of the trigger window or $64 \mu$ s later, ie $282 \mu$ s onwards. The pre-shutdown data represent $23 \%$ of our exposure in first data. Instead of throwing the pre-shutdown data away, it was decided to treat it as if it contains two NuMI beam windows, with a structure shown in figure 5.2. Cosmic background to $\nu_{e}$ analysis (see table 8.5) is reduced to a very small number, so the gain in exposure from including the pre-shutdown data far outweighs the penalty due to doubling of the cosmic background for this period. The livetime figure reported in table 5.1 accounts for the doubled time window in the pre-shutdown data.

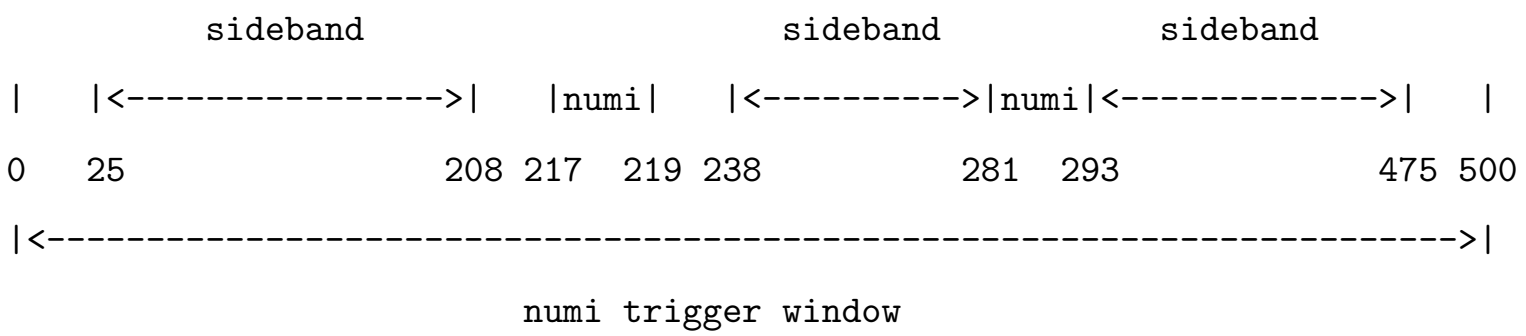

Figure 5.2: Diagram showing the timing sideband in NuMI trigger windows in preshutdown data. A second NuMI window is opened from 281 to $293 \mu$ s in order not to miss any neutrino data due to the $64 \mu$ s offset failure mode of TDUs.

\subsection{Simple Oscillations}

The size of the $\nu_{e}$ appearance signal in the far detector is a function of the oscillation parameters. Since it becomes tedious to quote all numbers for every likely oscillation scenario in all cases, in this document nearly all numbers are quoted on applying, what we call, simple oscillations. In this scheme, the $\mathrm{CP}$ violation phase $\delta=0$ and the oscillations are assumed to be maximal $\left(\theta_{23}=45^{\circ}\right)$. Additionally, matter effects are ignored (effectively, earth's crust density $\rho$ is set to 0 ), so the signal is independent of 
hierarchy. We also only consider the leading term in the oscillation probability :

$$
P_{\text {simp }}=\sin ^{2} \theta_{23} \sin ^{2} 2 \theta_{13} \sin ^{2}\left(\frac{\Delta m_{32}^{2} L}{4 E}\right)
$$

Recall that the two extreme oscillation scenarios for $\mathrm{NO} \nu \mathrm{A}$ are $\delta=3 \pi / 2$ for normal hierarchy $(\mathrm{NH})$ where the signal will be the largest and $\delta=\pi / 2$ for inverted hierarchy (IH), where the signal will be the smallest. The simple oscillations are in between these two extremes, closer to the $\delta=3 \pi / 2$, NH case. The results assuming these two extreme cases are also presented where appropriate. 


\section{Chapter 6}

\section{Simulation}

This chapter describes the simulation used in the first-analysis of the $\mathrm{NO} \nu \mathrm{A}$ data. Several improvements that have been made since have not been included here.

\subsection{Particle Simulation}

\subsubsection{Beam}

The $\mathrm{NO} \nu \mathrm{A}$ simulation chain begins with the simulation of hadron production and decay in the NuMI beamline. This simulation is done with FLUGG, which uses FLUKA2011 for simulating the physics of particle interactions and decays and GEANT4 to simulate the geometry, ie the environment that the particles interact in and with. The geometry consists of the graphite target and relevant elements of the target hall, the horns and the decay pipe. FLUKA simulates hadron production in the NuMI target and tracks them until they decay into neutrinos or are absorbed by the hadron monitors downstream. $\mathrm{NO} \nu \mathrm{A}$ beam simulation is similar to the one MINOS uses as described in $[27]^{1}$.

Hadron production simulation is a time consuming process. Since the decay of spin 0 hadrons is isotropic in their rest frame, the probability of the decay neutrino passing

\footnotetext{
${ }^{1}$ Note that $\mathrm{NO} \nu \mathrm{A}$ beam simulation does not have a $1 \mathrm{GeV}$ threshold for hadron tracking, since the detectors are off-axis and see a lower energy beam than MINOS.
} 


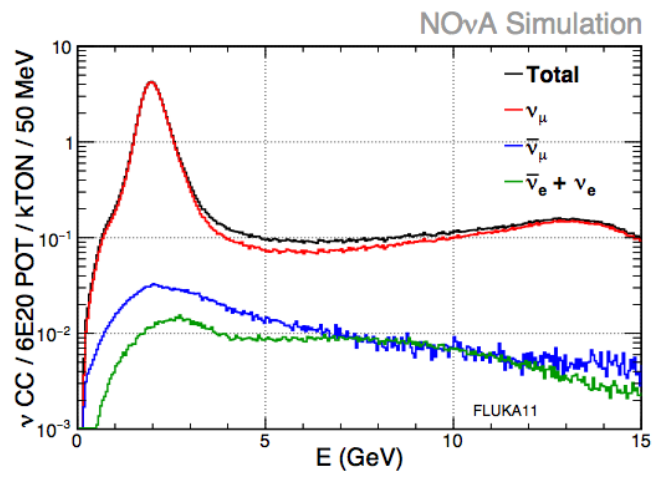

(a) Far Detector

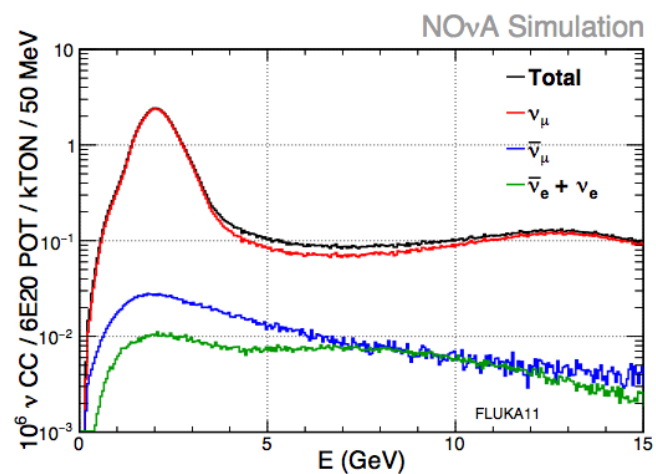

(b) Near Detector

Figure 6.1: FLUGG's neutrino flux prediction by flavor and sign for the $\mathrm{NO} \nu \mathrm{A}$ detectors in the forward horn current mode. The ND peak is broader since NuMI beamline is a line source of neutrinos as such a close distance. FD sees it as a point source.

through the detector is just the fractional solid angle that the detector subtends at the point of the hadron's decay. So it suffices to save kinematic properties of the neutrino parents in flux files that can later be used and reused to generate neutrinos to interact in the $\mathrm{NO} \nu \mathrm{A}$ detectors. The availability of neutrino parent information in flux files also allows us to reweight the parent, and therefore the resulting neutrino spectra, for the purpose of assessing beam related systematic errors.

\subsubsection{Neutrinos}

Interaction of beam neutrinos in the $\mathrm{NO} \nu \mathrm{A}$ detector environment is done with GENIE event generator [28] that reads in FLUKA generated flux files. GENIE uses Bodek and Ritchie version of the Relativistic Fermi Gas (RFG) nuclear model that has been modified to include short range nucleon-nucleon correlations. GENIE uses the flux and the total cross-section model to determine the energies of the neutrinos that will interact. It then uses interaction-specific cross-section models to decide which interaction type (QE, DIS etc) will occur and then the differential cross-section models to produce the final 
state kinematics. The nature of the final state particles and their kinematics is determined by the hadronization model. GENIE also simulates the interaction of final state hadrons while they are still inside the nucleus in a subpackage called INTRANUKE. The final state interaction (FSI) rates are derived from the free hadron cross-sections and the density of nucleons. Because these measurements are difficult to make, there are large uncertainties on these models which directly impact the observed energy of neutrinos in experiments.

Cosmic rays in $\mathrm{NO} \nu \mathrm{A}$ are simulated using the CRY (Cosmic RaY) generator [29]. It generates primary cosmic ray particles between $1 \mathrm{GeV}$ and $100 \mathrm{TeV}$ and secondaries between $1 \mathrm{MeV}$ to $100 \mathrm{TeV}$.

\subsubsection{Particles in Detector}

The geometry of the $\mathrm{NO} \nu \mathrm{A}$ detectors, detector halls and the surrounding earth is encoded in CERN's GDML (Geometry Description Markup Language) which is parsed by ROOT's geometry classes. Once GENIE and CRY create the particles from a neutrino interaction and cosmic showers, their propagation and energy loss in the $\mathrm{NO} \nu \mathrm{A}$ detector, and possible decays, are simulated by GEANT4 [30]. It simulates particle propagation step-by-step, and various process are categorized as those that occur at rest, or during a step or after a step is completed. Processes that happen continuously such as energy loss in media fall in the second category, decay at rest falls in the first and generation of secondary particles due to decay or multiple-scattering fall in the third. All possible process that can occur in these categories propose a step size based on their interaction length. GEANT takes the step which has the smallest length. Which physics processes are considered and how they are modeled is decided by the Physics List. In $\mathrm{NO} \nu \mathrm{A}$, we use the QGSP_BERT_HP list. This list consists of quark gluon string (QGS) model for modelling high-energy hadrons with Bertini cascade model for hadrons with less than $10 \mathrm{GeV}$ energy. In addition, it has a high-precision (HP) neutron model that tracks thermal neutrons $(<20 \mathrm{MeV})$ accurately. 


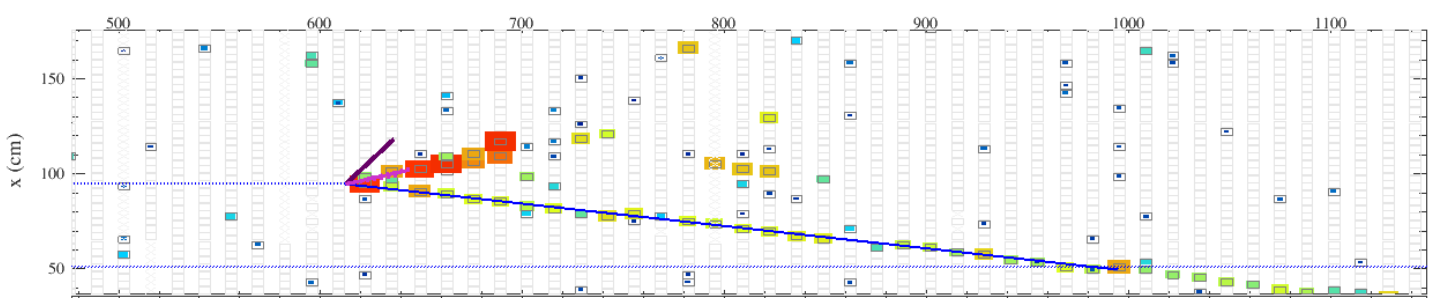

(a) Simulation of neutrino interactions with GENIE

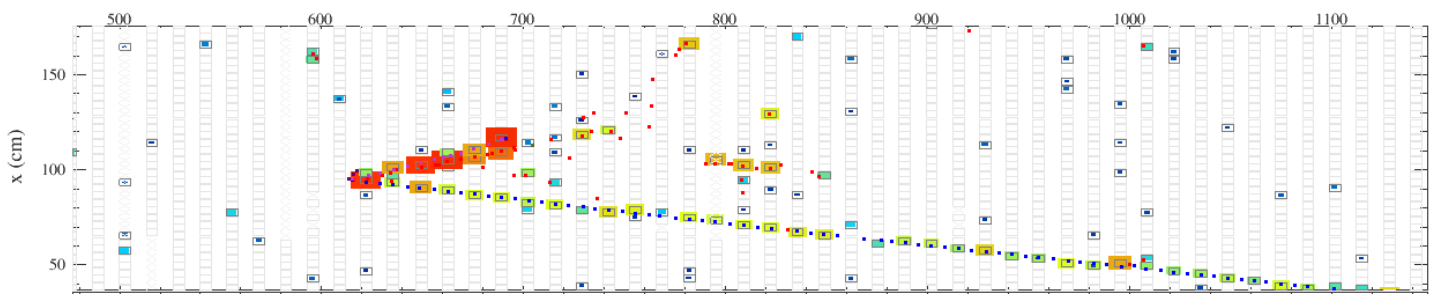

(b) Simulation of particle propagation in detector with GEANT

Figure 6.2: Particle simulation steps in $\mathrm{NO} \nu \mathrm{A}$. Figure 6.2a shows the output from GENIE. The lines are the momentum vectors of the generated particles. The dotted line is due to the incoming neutrino, while the solid lines are for the charged daughter particles. This information is relayed to GEANT that propagates these particles in the detector. The dots are the cells where the simulated particle deposited energy. The colored cells are energy depositions that, upon photon transport and readout simulation, were registered as above threshold hits. The scattered hits, with small charges, in the background are simulated noise for $500 \mu \mathrm{s}$.

\subsection{Detector Response Simulation}

\subsubsection{Photon Transport}

GEANT creates energy deposits that must be turned into photons that scatter, reflect and are absorbed by the fiber. A ray-tracing algorithm has been developed to compute the expected light collection rate ahead of time. It uses the measured $\mathrm{NO} \nu \mathrm{A}$ scintillator response, PVC reflectivity and the measured absorption spectrum of the fiber and the result is shown in figure 6.3. All the cells in a detector are treated identically at this 
stage, since the working assumption is that all cell to cell variations in scintillator and fiber response are taken out during calibration.

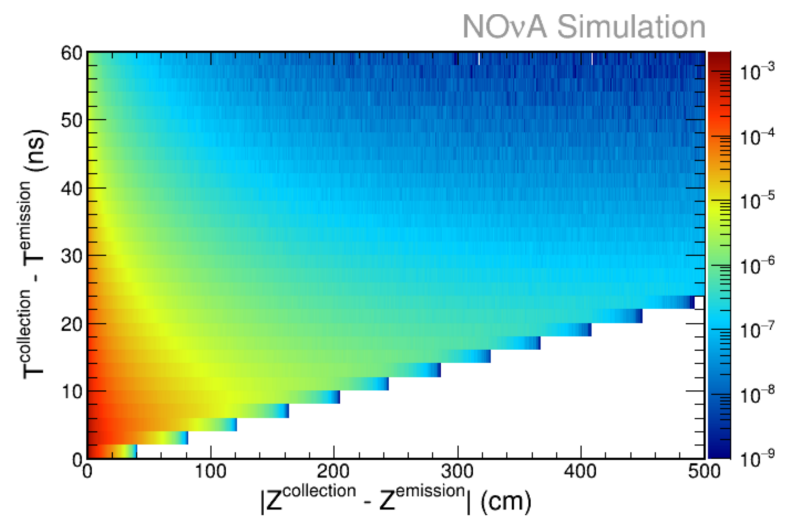

Figure 6.3: Light collection rate estimate from the ray tracing algorithm

Each side of the fiber loop gets half the collected light. The light loss in the fiber is simulated according to the average light attenuation measured in the fiber during detector construction. At this step, different cells in a module are treated differently, since the length of fiber in the manifold cover is different for each cell.

\subsubsection{Readout Simulation}

We model the APD to have a flat $85 \%$ quantum efficiency and a gain of 100 with $5 \%$ variation. The photo-electron pulse is shaped and smeared in time, to model the CR-RC circuit response in data as:

$$
f(t)=N_{p e} \frac{F}{F-R}\left(e^{-\left(t-t_{0}\right) / F}-e^{-\left(t-t_{0}\right) / R}\right)
$$

where $R$ and $F$ are respectively the rise and fall times, $t_{0}$ is the time at which the pulse occurred and $N_{p e}$ is the number of photo-electrons collected. The shaped pulse is distorted to simulate current and voltage variations (see [31]) and converted in ADC based on a pre-determined PE to ADC conversion factor from charge-injection studies. The baseline is determined from pedestal data and clock-ticks simulated to finally arrive 
at the $\mathrm{ADC}$ value of $A D C_{i}-A D C_{i-3}$. A hit is registered if this difference is above the threshold value for the cell. Threshold distribution is obtained from data from the two detectors and randomly sampled for simulation. For cells that have physics hits in them, the ADC trace is simulated for the full $500 \mu$ s window.

We also insert noise in cells with no physics hits in them. The noise rate and ADC distribution is derived from pedestal scan data from the two detectors.

\subsection{Tuning to Data}

To tune the simulation to the raw detector response, we use cosmic trigger data and simulation. The pulse height per cell, per path length through cell as a function of distance from the APD in simulation is pegged at the distribution from data to tune the absolute scintillator light level. The results of this tuning are shown in figure 6.4 for

both near and far detector. The peaks of the distribution match well while the modeling of saturation in $\mathrm{MC}$ does not quite agree with data.

Since the near detector beam data is not blinded and is high-statistics, it allows us to put our simulation to more varied and rigorous tests. Data-MC comparisons in the near detector revealed two major effects whose adequate simulation was missing from our MC. These are discussed in more detail below. 


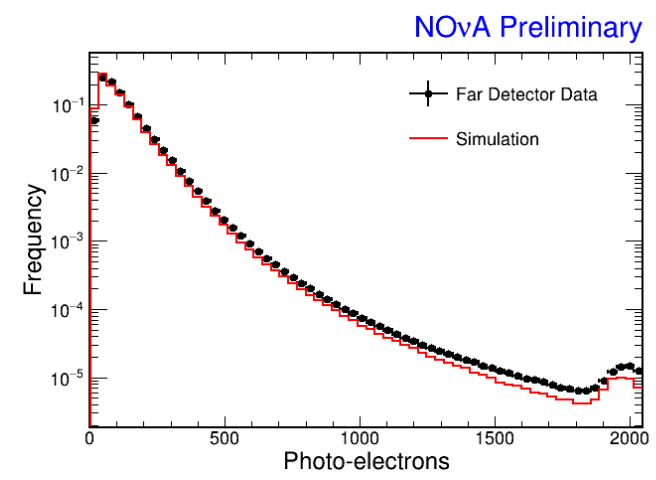

(a) FD

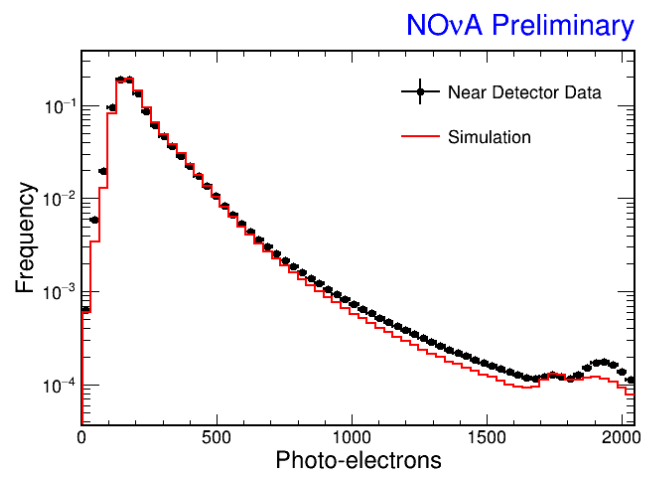

(b) ND

Figure 6.4: Comparison of raw pulse-heights in FD and ND cosmic ray data

\subsubsection{APD Sag Simulation}

APD sag discussed in 4.3.1 was thought to be a small effect and not included in the simulation of readout. But comparisons of path-length normalized pulse heights in muons in the near detector data showed a clear and non-negligible effect. This is shown in figure 6.5. If a muon leaves multiple hits in a plane, the $d(P E) / d x$ in the plane for the muon drops evidently. The drop observed is $1.87 \%$ of the total charge deposited on the APD, summed over all the triggered cells, and is consistent with the benchtop APD charge injection studies [32]. Including this effect in simulation brings the data and MC response in good agreement. And because for hits with physics activity in them, the trace is simulated for the full $500 \mu$ s window, we are also able to observe retriggering or flashing of APD in these cells.

\subsubsection{Scintillator Quenching}

It is well known that when the ionization density due to the passage of a charged particle through organic scintillators becomes very high, the light output of the scintillator is no longer proportional to the $d E / d x$ of the particle due to quenching of the primary fluorescence process. The parameterization of this suppression was put forth by Birks 


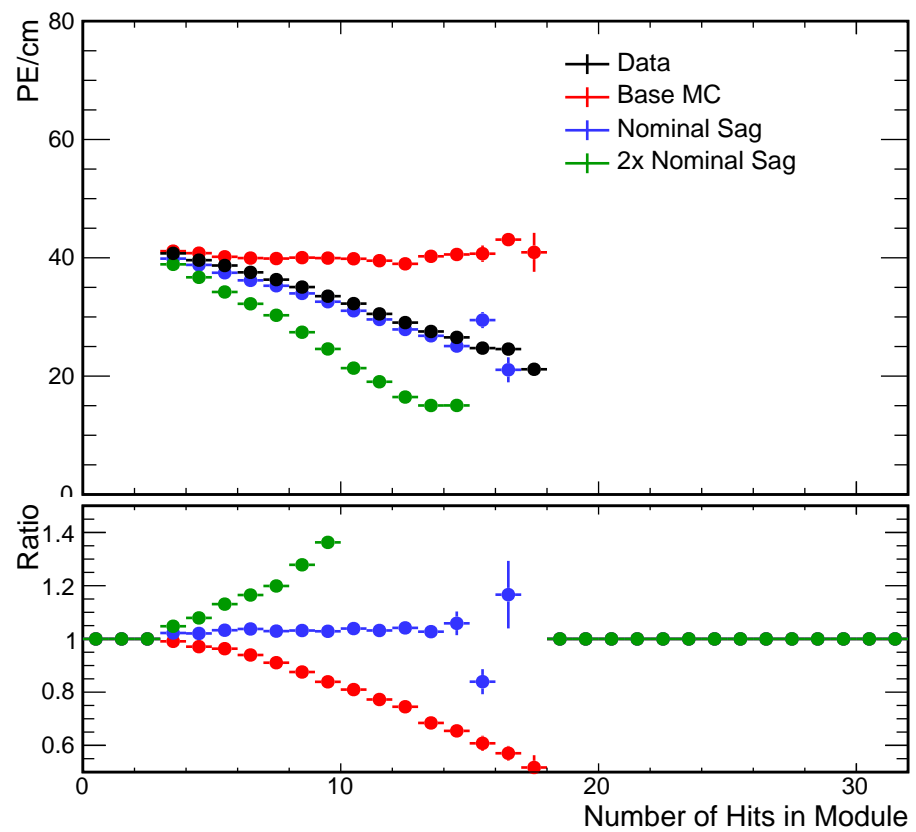

Figure 6.5: $\mathrm{PE} / \mathrm{cm}$ per plane measured in near detector data compared with MC with no sag, $1.86 \%$ sag and $2 \times 1.86 \%$. The bench top measurement of $1.86 \%$ appears to be representative of the data.

[33] as:

$$
\frac{d L}{d x}=S \frac{d E}{d x} \frac{1}{1+k_{B} \frac{d E}{d x}}
$$

where $d L / d x$ is the light output per path length for energy deposition per path length, $d E / d x . S$ is the absolute scintillator efficiency and $k_{B}$ is the Birks suppression constant. The value of $k_{B}$ depends on the type of scintillator and is of the order $10^{-2} \mathrm{~g} / \mathrm{MeV} \mathrm{cm}^{2}$. Scintillator quenching was simulated in $\mathrm{NO} \nu \mathrm{A} \mathrm{MC}$, but the magnitude of $k_{B}$ was too low. Comparison of $d E / d x$ of candidate protons in ND data with MC clearly showed an anomaly. This study was done with the shorter of the two tracks in a two-track sample of neutrino interactions, where the primary track was identified as the muon. In figure 6.6 the $d E / d x$ is plotted for the last six planes of the candidate proton tracks and shows that $\mathrm{MC} d E / d x$ is systematically higher than the observation in data. 

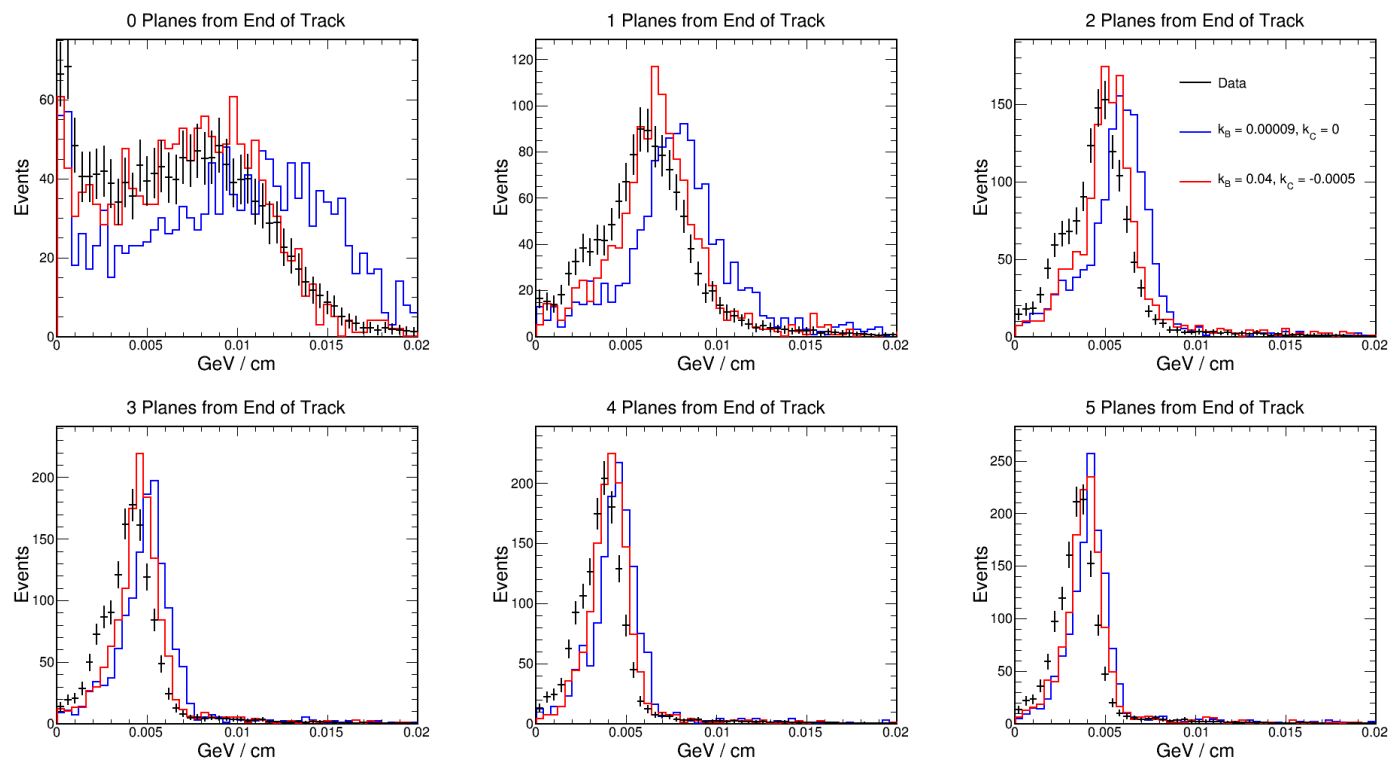

Figure 6.6: $d E / d x$ comparison between ND data and MC for last six planes of the candidate proton tracks. The schintillator was not quenched adequately (blue) and the $\mathrm{MC} d E / d x$ was consistently higher than data. Properly tuning the suppression parameters in the MC now gives good agreement (red) with data (black).

Our data is modeled well with an extension of the Birks' suppression law proposed by Chou [34], which has a second order correction term:

$$
\frac{d L}{d x}=S \frac{d E}{d x} \frac{1}{1+k_{B} \frac{d E}{d x}+c\left(\frac{d E}{d x}\right)^{2}}
$$

where $c$ is the Chou constant. Tuning $k_{B}$ and $c$ to ND data gives us values of 0.04 and -0.0005 respectively. With this large of a Birks suppression, the energy in the last plane would be over-suppressed; the negative contribution from the Chou constant remedies this over-quenching. These parameter values have been included in the final first-analysis simulation. 


\subsubsection{Diblock and Bad Channel Masking}

The first analysis dataset consists of data from a period when the detector was still under-construction or being commissioned. Since the data acquisition systems for diblocks are independent, we are able to turn off any particular diblock which needs hardware repairs, while taking data on the others. Because of this, the dataset consists of many different diblock configurations. This information about which diblocks are active during a run is stored in the database and is associated with the run number.

Also, the far detector consists of just over 344,000 cells. In any subrun, roughly $0.5 \%$ of them are masked off because of high noise levels or lower than acceptable data rate. The channel mask information is stored in the database on a subrun basis.

To make our simulation as close as possible to the real running conditions of the detector, the far detector MC used in the first-analysis predictions is generated with diblock configurations from data. How often each configuration is generated is weighted by the amount of POT collected in that configuration in data. The simulation also has the same channel masks applied as in data for both, near and far detectors. This means that the MC properly handles the changing fiducial mass and we are able to estimate

the fiducial volume dependent selection efficiency and arrive at a reasonable estimate of the expected signal counts for any set of oscillation parameters. 


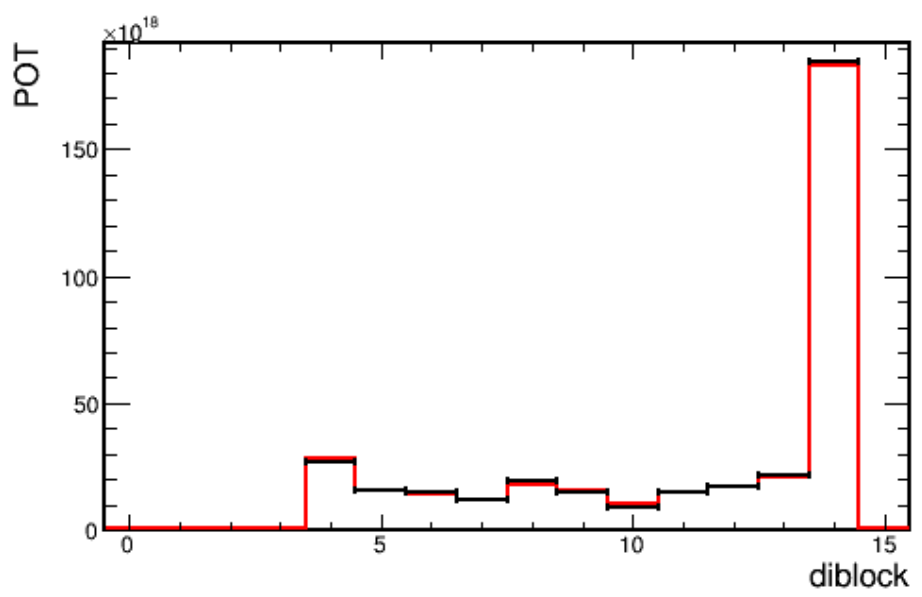

Figure 6.7: Relative exposure in each diblock configuration in data and simulation. Red is simulation and black is data. The simulation is area normalized to data.

\section{Summary}

$\mathrm{NO} \nu \mathrm{A}$ simulation chain uses GENIE, CRY and GEANT, which are third-party, generic particle simulation packages. The light propagation and front-end simulation is done using custom tools developed by the $\mathrm{NO} \nu \mathrm{A}$ collaboration. The simulation has been tested against far detector cosmic ray data and the neutrino beam data in the near detector. Several improvement have been made to the simulation to better model the detector conditions observed in data, including varying detector mass, which is crucial to arrive at a reasonable prediction. 


\section{Chapter 7}

\section{Event and Energy Reconstruction}

An above threshold charge deposit in a detector cell, or electronic noise that appears as such, is recorded as a hit. Since $\mathrm{NO} \nu \mathrm{A}$ cells are long tubes, a hit can only give 2-D information about the particle trajectory. The plane gives the $z$ coordinate and the cell gives the $x$ coordinate if it is vertical and $y$ coordinate if it is horizontal. To learn where along the depth of the cell the particle passed through, an $x$ view cell must be correlated with a cell from the $y$ view and vice versa. This is the task of reconstructionto associate hits in the detector that arise from the same particle and organize them into track or cluster objects, as appropriate.

Once the distance of the particle from the readout is determined, an attenuation correction to the pulse-height can be applied to derive the true energy that the particle deposited in the cell. This process of converting pulse-height into energy is handled by the calibration framework.

\subsection{Calibration}

Calibration in the NOvA detectors is done using cosmic ray data. A simple tracking algorithm is used to reconstruct the cosmic rays and several quality cuts are applied to ensure that the tracks belong to muons. The energy deposited in a cell belonging 
to the reconstructed track is normalized by the path-length in a cell and this channel response is then calibrated. Since path-length on a cell-by-cell basis can be difficult to estimate due to reconstruction effects, most of calibration uses tri-cells, that is, cellhits where both the adjacent cells in the plane were also triggered by the same cosmic ray as shown in figure 7.1. This selection ensures that the particle entered through the top wall and exited through the bottom wall in that cell and constrains the path-length to be cell-height, corrected by the direction cosine in that view.

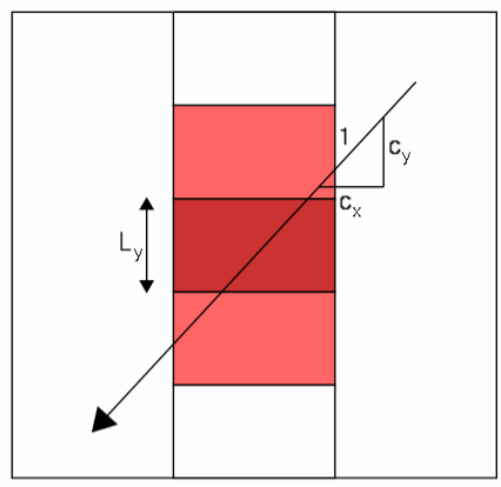

Figure 7.1: Selection of tri-cells associated with a track. The dark red cell is a tricell because its neighbors are triggered by the same cosmic ray. The path-length in cell is given by $L_{y} / c_{y}$

In many of the calibration procedures, $W$ is used to denote the depth in cell. $\mathrm{W}$ is aligned along the length of the cell and has a value of $0 \mathrm{~cm}$ at the center of the cell. Therefore, the range of $W$ for a FD cell is about $-775 \mathrm{~cm}$ (farthest from the APD) to $775 \mathrm{~cm}$ (closest to the APD).

\subsubsection{Attenuation Correction}

Light attenuates as it travels through the optical fiber in a NOvA cell. The attenuation correction converts the pulse-height recorded in ADC to corrected photo electrons (PECorr) units. An energy deposit expressed in PECorr is independent of the distance 
from the APD at which it was deposited. This calibration is done individually for each channel in the detector.

The $\mathrm{ADC} / \mathrm{cm}$ is recorded for a given cell as a function of $\mathrm{W}$ in a two-dimensional histogram. The histogram must have at least 5000 entries in it to be used for calibration. The profile of this histogram is constructed by taking the median value in each $\mathrm{W}$ bin and is fit to an exponential function of the form:

$$
y=C+A\left(e^{W / L}+e^{-W^{\prime} / L}\right)
$$

where $W^{\prime}$ is the longer path around the fiber, given by $(W+1.5 \times$ cell-length $)$ and $C$ is an arbitrary constant scale factor. Hits close to the beginning and the end of the cell exhibit different behavior than from the bulk of the cell. This is likely because in the bulk of the cell, most of the light that hits the white PVC cell walls is reflected back into the scintillator; the manifold cover at the top of the cell is composed of a black plastic which isn't nearly as reflective and results in greater light loss. To correct for this effect, a polynomial function is added to the above exponential form at the two ends of the cell. The roll-off is empirically determined to follow the following form:

$$
y= \begin{cases}1-\alpha_{R}\left(W-W_{R}\right)^{4} & : W>W_{R} \\ 1-\alpha_{L}\left(W-W_{L}\right)^{4} & : W<-W_{L}\end{cases}
$$

The full fit to each channel is a product of 7.1 and the above. The seven freeparameters from these two functions are stored in a database table for access by later reconstruction.

In some cells, large residuals are observed. This may result from fiber performance variation. In such cases, the exponential function does not correctly describe the response. To deal with this problem, a LOWESS (Locally Weighted Scatter plot Smoothing) fit is done on the residuals from the exponential fit to smooth the cell response. 

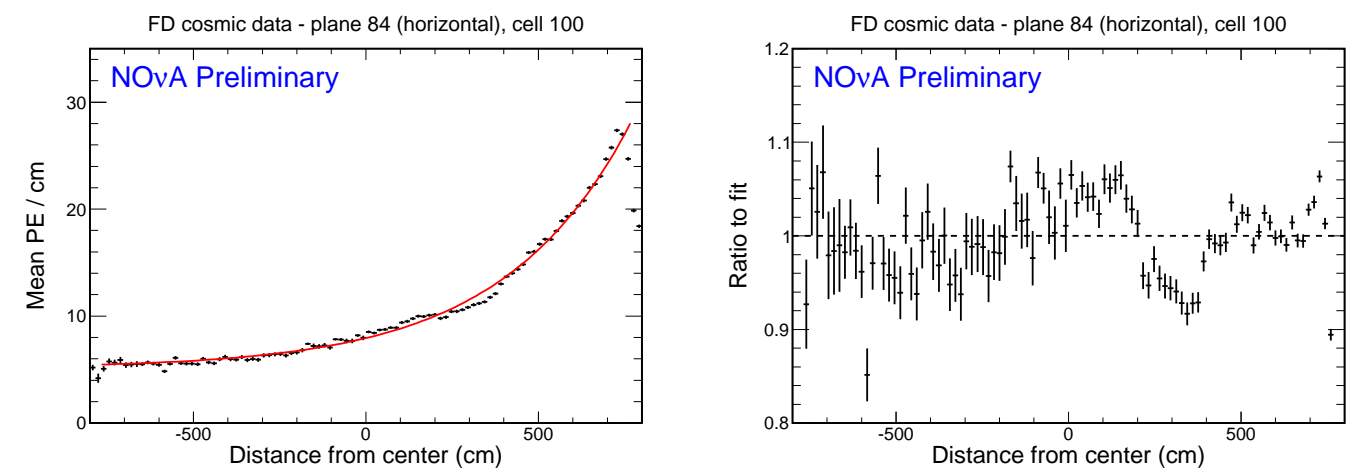

Figure 7.2: Attenuation correction of a far detector cell, and the residual of the fit

\subsubsection{Absolute Energy Scale}

The purpose of the absolute energy calibration is to convert the attenuation corrected response into energy units, ie $\mathrm{MeV}$ or $\mathrm{GeV}$. We use cosmic muons that enter from outside and stop in the detector for this because they deposit energy in a well-understood way, according to the Bethe-Bloch curve. The $d E / d x$ information in a $1 \mathrm{~m}$ long window on the track, $1 \mathrm{~m}$ away from the stopping point of the muon, shown in figure 7.3 , is used to measure the detector response, as the $d E / d x$ is approximately flat in this region. The corrected photo-electrons per length $(\mathrm{PECorr} / \mathrm{cm})$ distribution derived from this region is used to determine the scale factor between the data and simulation. That scale factor is then used to convert the PECorr/cm in data to $\mathrm{MeV} / \mathrm{cm}$ 


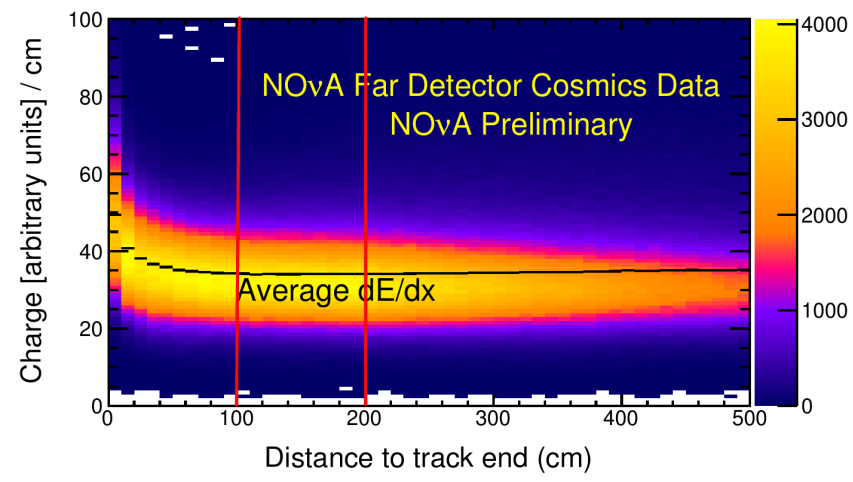

Figure 7.3: $d E / d x$ as a function of distance from the muon stopping point measured in the cosmic sample in the $\mathrm{NO} \nu \mathrm{A}$ far detector. The hits between the two red lines marking a $1 \mathrm{~m}$ long window, $1 \mathrm{~m}$ away from the muon end, are used for absolute calibration.
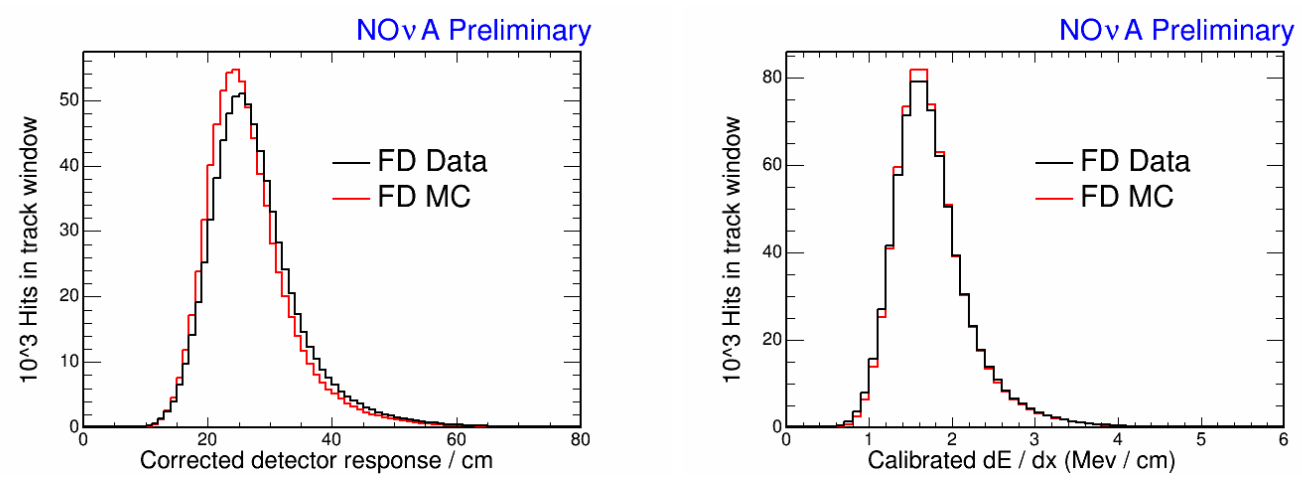

Figure 7.4: The attenuation corrected $d E / d x$ response is different between data and $\mathrm{MC}$ and this scale factor is used to determine the PECorr $\rightarrow \mathrm{MeV}$ conversion factor for data.

\subsubsection{Timing resolution}

The timing resolution is determined empirically for both the detectors. The sample used in this calibration is through-going muons from cosmics in the far detector and from neutrino interactions in the rock preceding the detector in the near detector. Hits are first corrected for time of flight of the muon and time of light propagation in the 
fiber to APD. After these corrections, all hits within the same DCM must occur at the same time, within the timing resolution. To compute the resolution, the post correction time difference between all pairs of hits in the same DCM is plotted as a function of the photoelectrons when the $\mathrm{PE}$ of the hits is within 25 photoelectrons of each other. The profile is fit with the following functional form to determine the timing resolution as a function of photo-electrons, $n_{\mathrm{pe}}$, in the cells:

$$
\sigma_{t}=\frac{p_{0}}{p_{1}+n_{p e}^{p 2}}+p_{3}
$$

where $\sigma_{t}$ is the timing resolution. The others are free parameters in the fit.
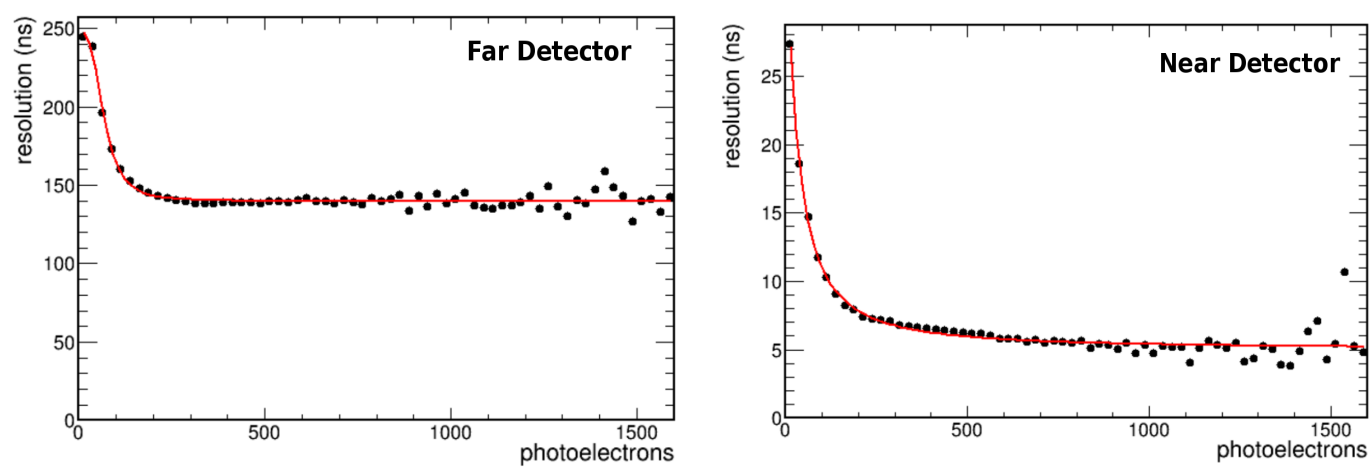

Figure 7.5: Timing resolution as a function of $\mathrm{PE}$ for the far and the near detector. Due to multi-point readout and faster sampling rate in the near detector, the timing resolution is much bettter than in the far detector.

\subsection{Reconstruction Steps}

The reconstruction tools used in $\nu_{\mu}$ disappearance and $\nu_{e}$ appearance analyses are somewhat different since the interactions produce very different signatures (see figure 7.6). The signature of a $\nu_{\mu}$ charged current interaction is a muon which produces a narrow track along its trajectory; a $\nu_{e}$ charged current interaction produces an electron which produces an electromagnetic shower that is more suitably reconstructed with clustering, rather than tracking algorithms. The following sections explain the reconstruction 
algorithms used in the $\mathrm{NO} \nu \mathrm{A} \nu_{e}$ analysis in some detail. The $\nu_{\mu}$ reconstruction chain is also described, but only briefly, since that is not the focus of this thesis.

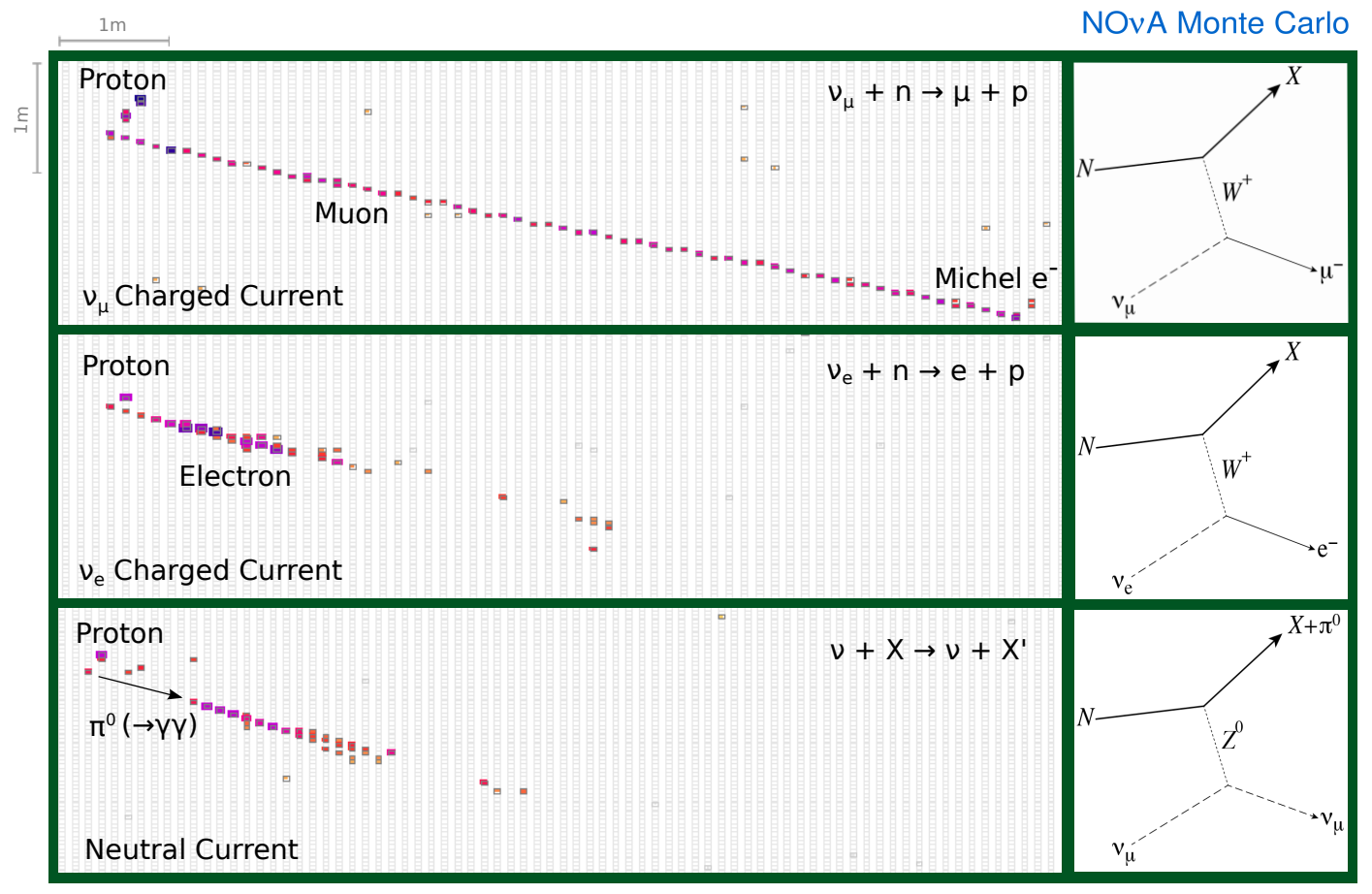

Figure 7.6: Profile of $\nu_{\mu}$ and $\nu_{e} \mathrm{CC}$ interactions. Muons form compact tracks while electrons produce wide showers. The bottom panel shows a particular, in no way typical, neutral current interaction that acts as a background to the $\nu_{e}$ appearance analysis due to the presence of EM showers.

The raw data coming out of the detector is organized into cellhits, that contain plane, cell, time and charge information about the hit. In addition to hit time, the timing resolution for the hit, based on its charge and determined by the procedure described in section 7.1.3 is also saved. Hits that are spatially and temporally correlated with each other are clustered together into slices. A slice in $\mathrm{NO} \nu \mathrm{A}$ is treated as an independent, self-contained interaction and all further reconstruction, for $\nu_{e}$ as well as $\nu_{\mu}$ analyses, is 
done on hits that belong within the same slice ${ }^{1}$.

The process of reconstructing a neutrino interaction within a slice for the $\nu_{e}$ analysis is split into three separate algorithms. The first is a two-point Hough Transform which identifies lines that serve as seeds. The next step uses the lines to reconstruct a neutrino interaction vertex using an Elastic Arms algorithm. The third step uses the Hough lines and the vertex information to cluster hits in the slice into prongs. For the $\nu_{\mu}$ analysis, the hits inside a slice are fitted into tracks using a Kalman Filter algorithm.

If the reconstruction works as designed, a slice corresponds to a single neutrino or cosmic ray interaction and each prong or track within the slice corresponds to a separate particle resulting from the interaction.

\subsection{Slicing Algorithm}

Slicing in $\mathrm{NO} \nu \mathrm{A}$ is based on the DBSCAN (Density-Based Spatial Clustering of Application with Noise) algorithm [35] which groups together points that are closely packed in some parameter space, marking points that lie alone in low density regions as noise.

In the DBSCAN algorithm, if a point $p$ contains more than a certain minimum number of points, MinPts, within a distance $\epsilon$ from itself, it is considered to be a core-point; the points within its $\epsilon$ neighborhood are its neighbors and are said to be directly-reachable from $p$. The points on the edges of clusters do not have the minimum number of hits in their $\epsilon$ neighborhood but are neighbors of core-points and are known as border points. A border point $q$ is reachable from a core point $p$, if all the points that connect $q$ to $p$ are directly-reachable from $p$, ie are core-points themselves. Points that belong to neither of these categories are loners and are treated as noise.

Clustering begins by scanning all points and calculating the number of points in their $\epsilon$ neighborhoods. If a core-point is found, a cluster is formed by finding all the

\footnotetext{
${ }^{1}$ There are some exceptions where hits outside of a slice are of interest, for instance in Michel electron finding algorithms
} 
points that are directly-reachable from the seed point. The expansion of the cluster terminates when all the branches end in a border point. The algorithm then returns to the original list of points and finds the next core-point that is not yet assigned to a cluster.

In this algorithm, $\epsilon$ and MinPts are free parameters that are tuned based on the density of clusters and noise in the particular problem. One is also free to define the distance function based on which it is determined if two points are each other's neighbor or not. In $\mathrm{NO} \nu \mathrm{A}$, we use the following definition of the distance function for two hits in the same view:

$$
D=\left(\frac{\Delta T-\Delta \vec{r} / c}{T_{\text {res }}}\right)^{2}+\left(\frac{\Delta Z}{D_{\text {pen }}}\right)^{2}+\left(\frac{\Delta X o r Y}{D_{\text {pen }}}\right)^{2}+\left(\frac{P E_{p e n}}{P E}\right)^{5}
$$

$T_{\text {res }}$ is the timing resolution of the two hits added in quadrature. $\Delta \vec{r}$ is the 2-dimensional distance between two hits. $\Delta Z$ and $\Delta X$ or $Y$ are the one dimensional distances between the hits in $\mathrm{Z}$ and $\mathrm{X} / \mathrm{Y}$ directions. The $P E$ in the last term is a sum in quadrature of the number of photoelectrons in the two hits. Since hits due to electronic noise are typically low in energy, this term works to suppress addition of noise into the clusters. $D_{\text {pen }}$ and $P E_{\text {pen }}$ are free parameters. The term is raised to the fifth power because the noise spectrum falls off as $P E^{-2.5}$. For hits that are not in the same view, $\triangle X$ or $Y$ is 0 and the denominator in the $\Delta Z$ term is smaller, since ideally hits would be in adjacent planes. The free parameters in slicer are tuned separately for the near and far detectors to meet the challenges of different event rates. The requirement from slicer is that each slice should contain a single interaction and it should contain all of it. We define two variables, efficiency and purity, shown in fig. 7.8 , to measure the performance of slicer. The efficiency is a measure of the fraction of hits from an interaction that are contained in a slice and purity is the fraction of hits in a slice that come from the leading interaction in the slice. All the slicer free-parameters have been tuned to maximize the number of slices that score high on both efficiency and purity. 

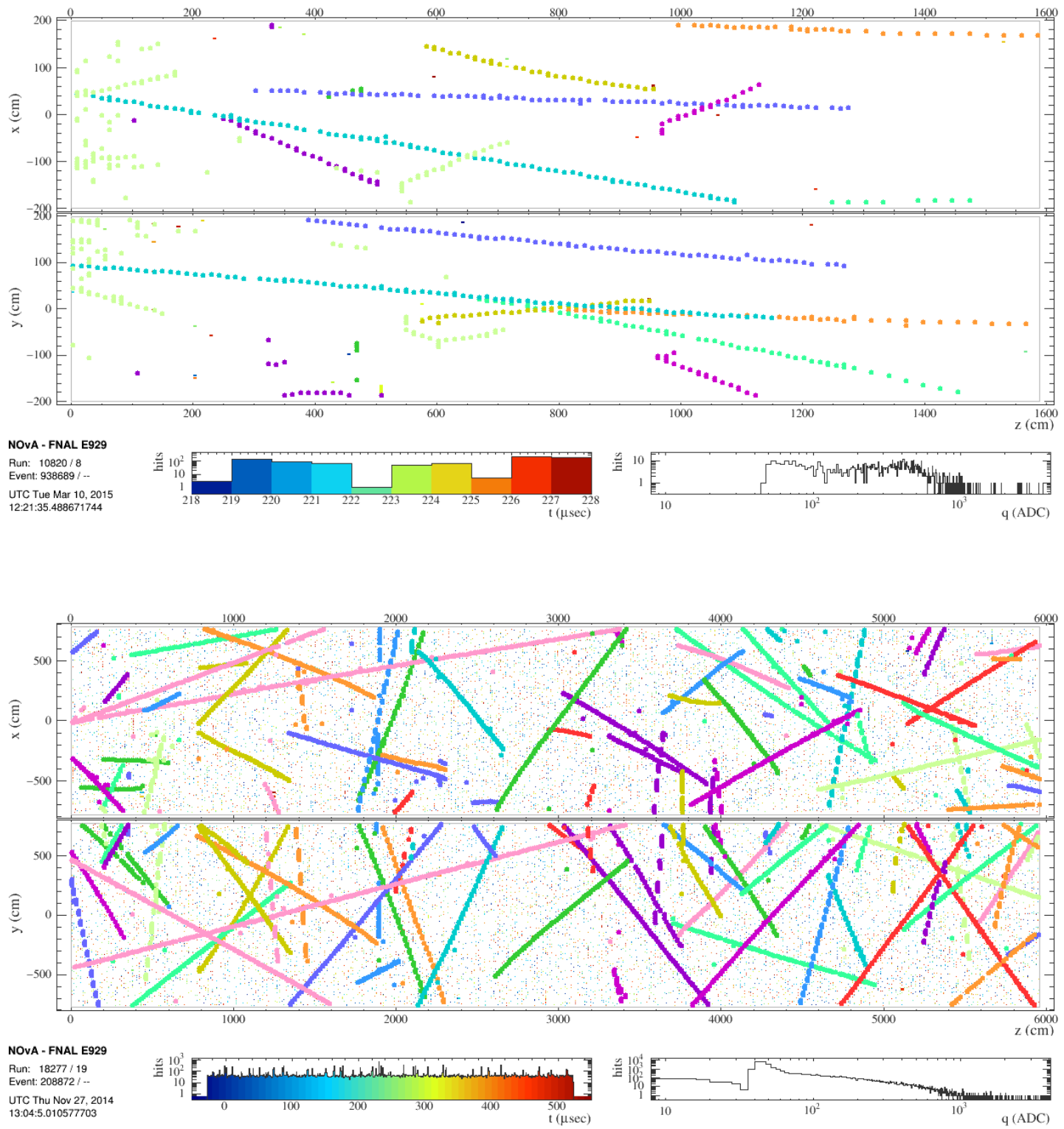

Figure 7.7: A near detector spill where the display is zoomed in on the $10 \mu s$ time window to display neutrino activity and a far detector $500 \mu \mathrm{s}$ time window shows cosmic rays. The dots in the same color indicate hits that have been clustered together in the same slice by slicer. 

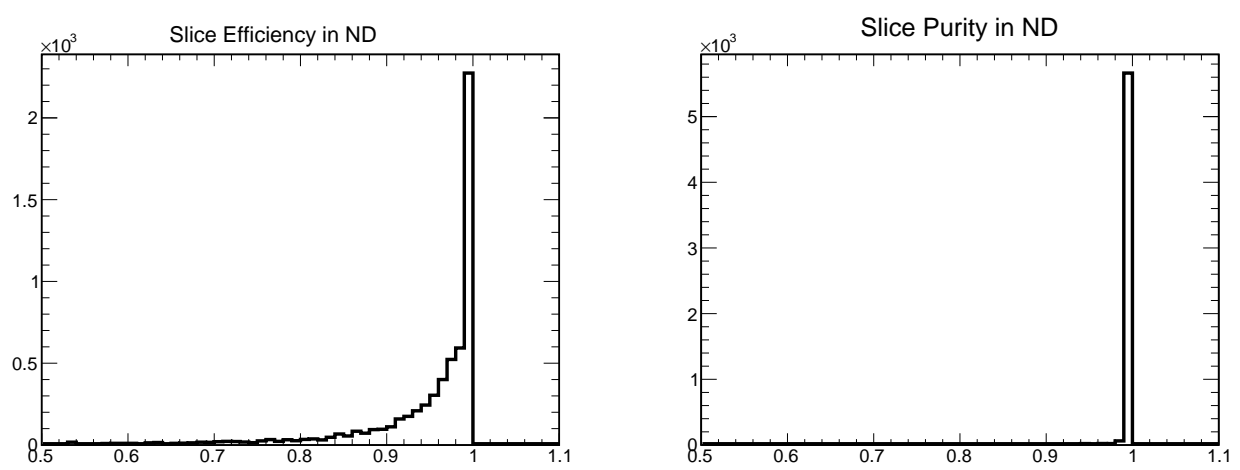

Figure 7.8: Efficiency and purity of slices in ND simulation. Over $80 \%$ slices have $>90 \%$ efficiency and over $95 \%$ have $>90 \%$ purity.

\section{$7.4 \quad \nu_{e}$ Reconstruction}

\subsubsection{Hough Transform}

The first step in the $\nu_{e}$ reconstruction chain is to identify lines that represent features in a slice. We use a modified Hough Transform [36] to achieve this end. In this algorithm, lines are parameterized in a polar space $(\rho, \theta)$ in order to naturally deal with vertical lines. The lines are fit separately in the $\mathrm{XZ}$ and $\mathrm{YZ}$ views. The line passing through each pair of points in the slice is recorded with a Gaussian smear vote

$$
\begin{aligned}
\text { vote } & =e^{-\frac{\left(\rho-\rho_{0}\right)^{2}}{2 \sigma \rho^{2}}} e^{-\frac{\left(\theta-\theta_{0}\right)^{2}}{2 \sigma \theta}} \\
\sigma_{\rho} & =\frac{3}{\sqrt{12}} \\
\sigma_{\theta} & =\frac{3}{d \sqrt{6}}
\end{aligned}
$$

where $d$ is the distance between the two hits. $3 \mathrm{~cm}$ is roughly the width of the cell and $\sigma_{\rho}$ is just the uncertainty due to a top-hat distribution. Pairs of hits that belong 

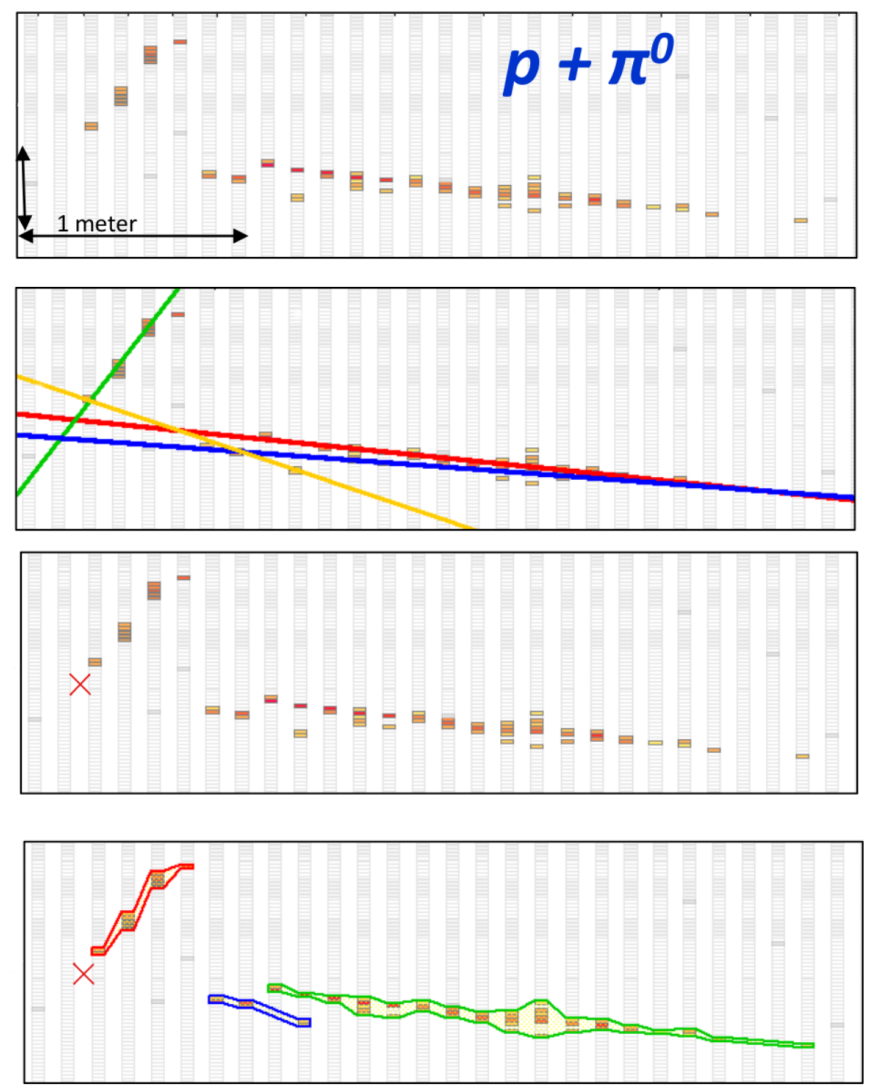

Figure 7.9: $\nu_{e}$ reconstruction chain. A neutrino interaction is first isolated into a slice of its own. Hough transform then finds global event features in terms of lines. Elastic arms uses hough lines to fit the event vertex. Fuzzy-K finds tracks that arise from it.

to true lines produces peaks in the Hough space of $(\rho, \theta)$ and can be grouped together into a line whose $\rho$ and $\theta$ are represented by a weighted mean around the peak. Peaks in the Hough-space that fall below a certain threshold are not reconstructed into a line. The current threshold is set at the average bin height in the Hough space for the slice. We use an iterative line finding process, such that the points that belong to the most prominent Hough line are removed from the list in the next iteration to create a new Hough map. This ensures that very prominent lines in a slice do not interfere with the reconstruction of the less obvious ones that also arise from physics, for instance short 
proton tracks.
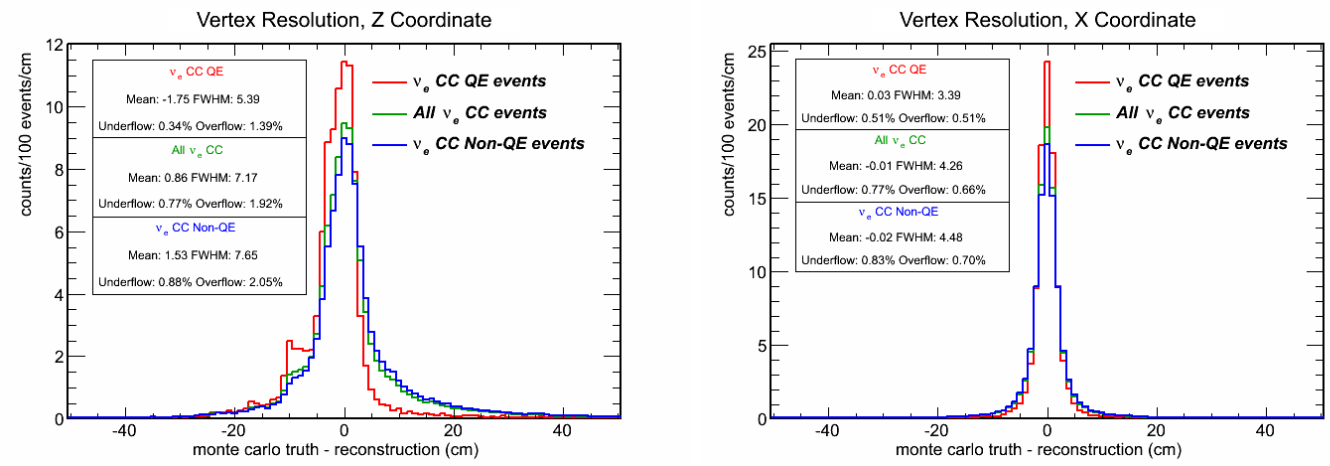

Figure 7.10: Resolution of the reconstructed vertex for $\nu_{e}$ charged current events measured with respect to the true neutrino interaction point. The resolution in the $x$ and $y$ directions are identical, so only one is shows.

\subsubsection{Elastic Arms Vertex}

The assumption of the version of Elastic Arms reconstruction used in $\mathrm{NO} \nu \mathrm{A}$ is that all activity in a slice has a common origin, the event vertex, and prongs or tracks, or arms extend from this vertex. The algorithm uses Hough lines as seeds for finding the global event vertex and arm directions. In an Elastic Arms algorithm [37], otherwise known as the method of deformable templates, once an event vertex is found, the arms that reconstruct the particles may be deformed or adjusted to better represent the event. The seed vertex and arm directions are assessed using the following energy function

$$
E=\sum_{i}^{N} \sum_{a}^{n} V_{i a} d_{i a}+\lambda \sum_{i}^{N}\left(\sum_{a}^{n} V_{i a}-1\right)^{2}+\frac{2}{\lambda} \sum_{v}^{n} D_{a}
$$

The first term is a measure of how well the arms describe the hits and the second is a penalty term for hits that are not assigned to any arm. The third term, which is specific to $\mathrm{NO} \nu \mathrm{A}$ 's implementation of this algorithm, assigns a penalty to arms that start more than a distance $\lambda_{v}$ away from the event vertex, where $\lambda_{v}=7 / 9 X_{o}$, the photon radiation length. 
In the first term, $d_{i a}$ is the distance of the $i$ th hit from the $a$ th arm, normalized by the detector spatial resolution. $n$ and $N$ are the total number of arms and hits in slice, respectively. $V_{i a}$ is a measure of the strength of association of the $i$ th hit with the $a$ th arm, and is given by:

$$
V_{i a}=\frac{e^{-\beta d_{i a}}}{e^{-\beta \lambda}+\sum_{b=1}^{n} e^{-\beta d_{i b}}}
$$

The factor $e^{-\beta \lambda}$ parameterizes the likelihood that the hit is noise. The parameter $\beta$ represents the range of influence of the arms and can be viewed as inverse of temperature. All the vertex seeds and arm directions are scanned and the set that minimize eq.7.3 is used in the final minimization step using root's MINUIT class. The fit procedure begins at low values of $\beta$ to avoid local minima in the energy function, and $\beta$ is gradually tuned up to close-in on the final event vertex.

\subsubsection{Fuzzy-k Prong Reconstruction}

While Elastic Arms algorithm works well to find the event vertex, its ability to deduce the correct number of arms or prongs in the event, and correctly associate hits with them is found to be inadequate. For this reason, Fuzzy-k follows Elastic Arms in the reconstruction chain and is designed to cluster hits that arise from different particles into separate prongs. Fuzzy-k clusters hits separately in the $\mathrm{XZ}$ and $\mathrm{YZ}$ views, so it begins with a 2-D problem. The algorithm treats the vertex from Elastic Arms as the origin of the event and assumes that all particles, ie prongs, appear as peaks of energy distributions in the angular space viewed from the event vertex, thereby reducing the problem to a 1-D problem.

The module is based on a possibilistic Fuzzy-K Means [38, 39] clustering algorithm. An extension of this algorithm [40] allows its application to situations where the total number of clusters is unknown to begin with. The method allows a hit to belong to more than one cluster and therefore, the boundaries of a cluster are fuzzy. The term possibilistic refers to the fact that the sum of a cell's membership across all clusters is 
not required to be 1 . This allows for treating the outlier hits as noise, such that their overall cluster membership is 0 .

The reconstruction begins by representing the vectors from the event vertex to each cellhit in a slice as an angle wrt to the $z$ direction. An angular uncertainty, $\sigma$, modeled after the multiple scattering of 1-2 GeV muons, is associated with each cellhit based on its distance from the vertex. Seeds for prong finding are set at angles of local maxima in cell density in the angular space, using a density matrix $w$ :

$$
\begin{aligned}
w_{k} & =\sum_{i}^{n} e^{-\left(\frac{\theta_{i}-\theta_{k}}{\sigma}\right)^{2}} \\
\text { with } \quad \theta_{k} & =-\pi+\frac{k * \pi}{180}
\end{aligned}
$$

where $\mathrm{k}$ is varied between 0 and 360 in steps of 1 . To assign cell membership, the angular separation between the $i$ th cluster core (defined as a line passing through the vertex at the seed angle) and the $j$ th hit is calculated:

$$
d_{i j}=\left(\frac{\theta_{j}-\theta_{i}}{\sigma_{j}}\right)^{2}
$$

The numerator is bounded in $[-\pi, \pi]$. The cluster membership assigned is:

$$
\mu_{i j}=e^{-\frac{m d_{i j} \sqrt{a}}{\beta}}
$$

$a$ is the number of clusters in the slice. $m$ is a measure of fuzziness of clusters and is a tunable parameter that we have set to 2 . If it is set to 0 , a hit can belong to only a single cluster. $\beta$ represents the expected spread of hits around the cluster center and is set to 4 . The cluster centers are then updated with:

$$
\theta_{i}^{\prime}=\theta_{i}+\frac{\sum_{j}^{n} \frac{\mu_{i j}}{\sigma_{j}^{2}}\left(\theta_{j}-\theta_{i}\right)}{\sum_{j}^{n} \frac{\mu_{i j}}{\sigma_{j}^{2}}}
$$

This process is iterated until the angular separation between the new cluster centers and the previous iteration is less than a pre-defined tolerance value. 

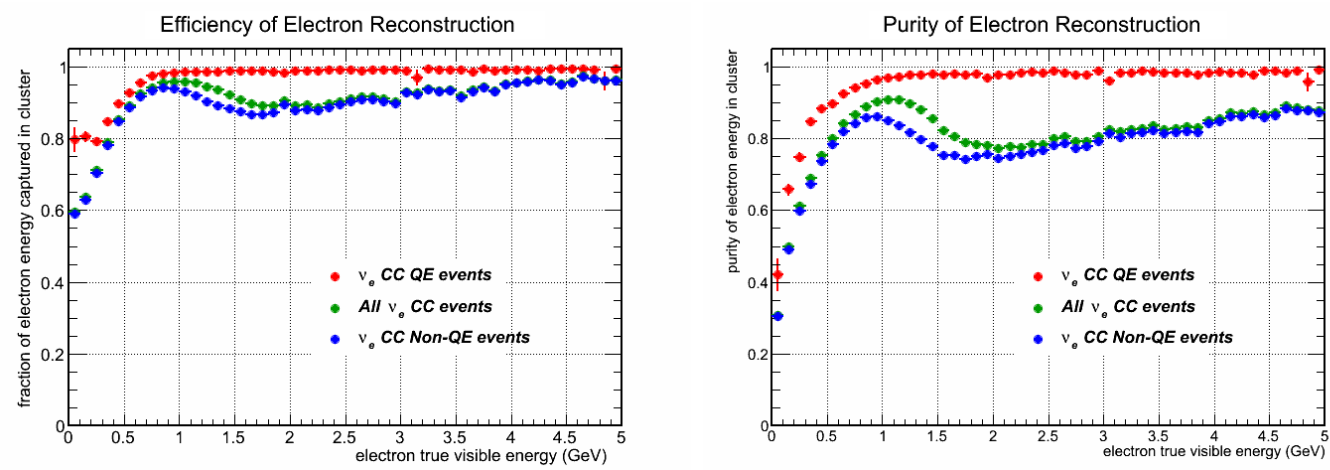

Figure 7.11: Efficiency and purity of reconstructed Fuzzy-K prongs that correspond to true electrons are $>80 \%$ for the region of interest for $\nu_{e}$ appearance analysis. The drop in efficiency and purity is due to the rise in the non-quasielastic modes of neutrino interaction. These produce significant hadronic showers that can contaminate the electron showers and make clustering difficult.

The final task is to match $2-\mathrm{D}$ clusters in $\mathrm{XZ}$ and $\mathrm{YZ}$ views to form $3-\mathrm{D}$ prongs. To do this, a 2-D cluster in one view is matched with each of the 2-D clusters in the other view. The cumulative energy as a function of distance from the prong start is computed for the prongs in each of the two views. If the 2-D prong is correctly matched to its counterpart in the other view, the energy profile should be very similar between the two views. To find the best match for the prong, a Kuiper metric, $K=\min \left(D^{+}, D^{-}\right)$ is used, where $D^{+}$and $D^{-}$are the largest negative or positive distances between the profiles.

\subsection{4 $\nu_{e}$ Event Energy Reconstruction}

The $\nu_{e}$ energy reconstruction used for the first analysis is very simple. Since NO $\nu \mathrm{A}$ is a calorimeter with $65 \%$ detector mass in scintillator, we would expect close to $65 \%$ of the true energy to be visible in the detector. In reality, the visible energy fraction is lower than that. Threshold effects and low energy particles suppress the visible energy to about $56 \%$. The rest of the energy is deposited in dead material, ie PVC, and is not 
visible.

To reconstruct the $\nu_{e}$ energy, we sum up the calorimetric energy of all the hits in a slice and scale it by a factor of $1.78(=1 / 0.56)$. This factor has been determined from the simulation of charged current interactions of electron neutrinos and yields an energy resolution of $\sim 10 \%$. More sophisticated energy reconstruction algorithms that treat the hadronic and electromagnetic energies separately are also available, but the resulting energy resolution is comparable to this simpler method, therefore we decided to not introduce unnecessary complexity.

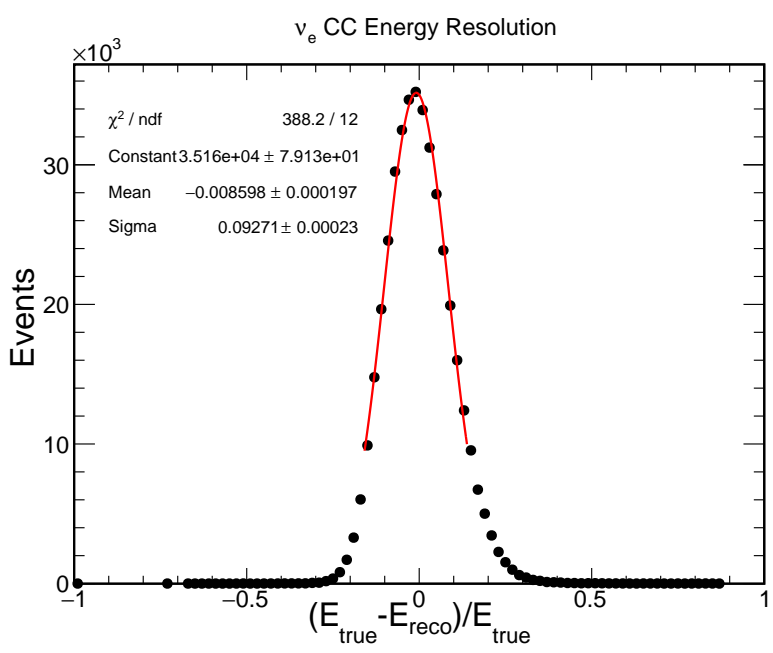

Figure 7.12: Reconstructed energy resolution of preselected $\nu_{e}$ charged-current interactions.

\section{5 $\nu_{\mu}$ Reconstruction Chain}

$\nu_{\mu}$ reconstruction uses a tracker based Kalman Filter [41] in order to accommodate tracks that curve due to multiple scattering of particles. Tracking first begins by assuming that all adjacent hits in a slice are tracks. A Kalman Filter subroutine is used to propagate tracks forward, plane by plan. Hits that are consistent with the track are added to it, 
while others are ignored. The consistency of a hit with the developing track is measured in terms of the change in $\chi^{2}$ of the track upon inclusion of the hit. If the hit is added, the track fit is updated by performing a weighted average fit over the hits. These tracks are formed separately in $\mathrm{XZ}$ and $\mathrm{YZ}$ views. Once all the 2-D tracks are found, a process to merge them into 3-D track is run. The merging mechanism relies on the tracks in the two views arising from the same particle to have similar start and end points in the $z$ direction within some tolerance.

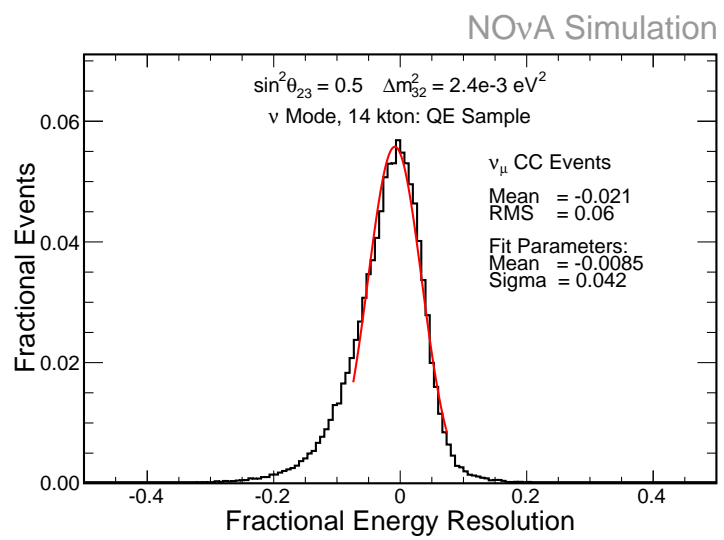

Figure 7.13: Muon neutrino energy resolution for quasi-elastic CC interactions.

To identify $\nu_{\mu}$ CC interactions, each reconstructed Kalman track is tested for likeness to a muon. A k-nearest neighbor algorithm based identifier named ReMId (Reconstructed Muon ID) has been developed for this purpose. It uses log-likelihood based on $d E / d x$ along the track in the longitudinal view as an input variable. The main background to muons are charged pions that are also minimum ionizing. However, pions may interact strongly and undergo hard scatters which distinguish them from muons. So length of the track and the scattering likelihood are also used as inputs.

The energy of the muons in $\nu_{\mu} \mathrm{CC}$ candidate interactions is computed from range. The hadronic energy in the event is taken to be the sum of the calorimetric energy of all the hits that do not belong to the muon track, corrected by a scale factor to account for energy deposition below threshold or in dead material. The muon neutrino energy 
is a sum of the muon and the hadronic energies. 


\section{Chapter 8}

\section{$\nu_{e}$ Event Selection}

For the selection of $\nu_{e}$ appearance signal and rejection of beam and cosmic backgrounds, we use a combination of simple cuts and more complicated particle identifiers. The simple cuts are aimed at rejecting low quality data due to occasional electronic failures and to exploit some obvious topological differences between signal and background events. The intent is to keep selection cuts identical between the near and the far detectors, which helps minimize systematics on the far over near ratio. But, in some cases, the vastly different sizes and data rates in the two detectors force small differences.

In section 8.1 of this chapter, we focus on selection cuts. The $\nu_{e}$ analysis has two main PID algorithms, LID and LEM. The LID algorithm builds on the reconstruction chain discussed in chapter 7 and section 8.2 takes a detailed look at this algorithm as LID is the PID used for this thesis.

\section{1 $\nu_{e}$ Selection Cuts}

\subsubsection{Data Quality Cuts}

Data quality cuts ensure that the detector and beam were in a reliable state at the time of data taking. 


\section{Beam Quality}

Beam quality cuts are applied on a spill by spill basis. The information on beam related metrics is hosted centrally in the intensity frontier database (IFDB) from where it can be retrieved by all the experiments on the NuMI beam. We apply the following cuts to both, near and far detector spills:

1. The event time recorded by $\mathrm{NO} \nu \mathrm{A}$ must be within $0.5 \mathrm{~s}$ of the spill time as recorded in the IFDB (note that the spills are $1.33 \mu \mathrm{s}$ apart)

2. Number of protons on target (POT) in the spill must be greater than $2 \times 10^{12}$. Spills with POT smaller than this are usually used for target scans and do not guarantee good beam.

3. The horn current, in the forward horn current configuration, must be between -202 and $-198 \mathrm{kA}$

4. Beam spot position requirements: $0.02 \mathrm{~mm}<x, y$ positions $<2.00 \mathrm{~mm}$

5. Beam spot size requirements: $0.57 \mathrm{~mm}<x, y$ widths $<1.58 \mathrm{~mm}$

The beam quality cuts remove less than $1 \%$ of the POT collected in the first-analysis datasets.

\section{Subrun Quality}

The subrun quality metrics are designed to remove data in case of significant or repeated failures of hardware during the span of a subrun. For a subrun to pass these cuts, it must have a livetime of at least 1 second. It must have at least two consecutive good diblocks in FD and all good diblocks in ND. A diblock is considered good if all the DCMs in it are operating optimally, that is, they have fewer than 9 misbehaving APDs. An APD is good if less than 7 of its pixels have a hit rate outside an acceptable range of $10^{0.5} \mathrm{~Hz}$ to $10^{3.5} \mathrm{~Hz}$. Response of channels with hit rates outside of this range is 
found to be unreliable. They are masked off and information from them is not visible to further offline analysis. These bad channel masks are generated on a subrun basis.

In addition, we require MIP (minimum ionizing particle) hit rate and reconstructed slice rate normalized by the number of active channels to be in a nominal range. The cut values on these metrics are different for ND and FD due to very different data rates.

In $\mathrm{FD}$, the hit rates are dominated by cosmics while in $\mathrm{ND}$, due its location underground, nearly all the hits come from the beam. The ND subruns have an additional cut to monitor the location of the beam peak in the $500 \mu s$ window.

In the first data-taking period, close to $11 \%$ of the total POT is lost to subrun quality cuts in the FD, and about $8 \%$ in the ND. The first data overlap with the commissioning period for both detectors when the failure rate of front-end electronics or other detector systems was high and the electronic parameters were still being optimized. The current FD data-taking is significantly more stable and we disqualify less than $1 \%$ of the data due to subrun quality cuts.

\section{Spill Data Quality}

While subrun cuts are essential for weeding out data where significant failures occurred, they are not fine enough to pick up on more local and smaller duration failures that nevertheless undermine the quality of reconstruction and identification. For this purpose a set of spill quality cuts has been designed. Events that fail spill quality cuts are not analyzed and the POT corresponding to such events is not counted towards our exposure.

Because of certain failures in the DAQ, there are short durations of time, typically lasting a few seconds, ie over several spills, when a subset of DCMs fail to report any data at all. This can be troublesome, because if hits from a DCM on the edge of the detector are missing, a cosmic ray entering from outside can appear to be contained. For this reason, we reject data from spills where any of the active DCMs is missing, ie reports no hits over the full $500 \mu$ s trigger window. 


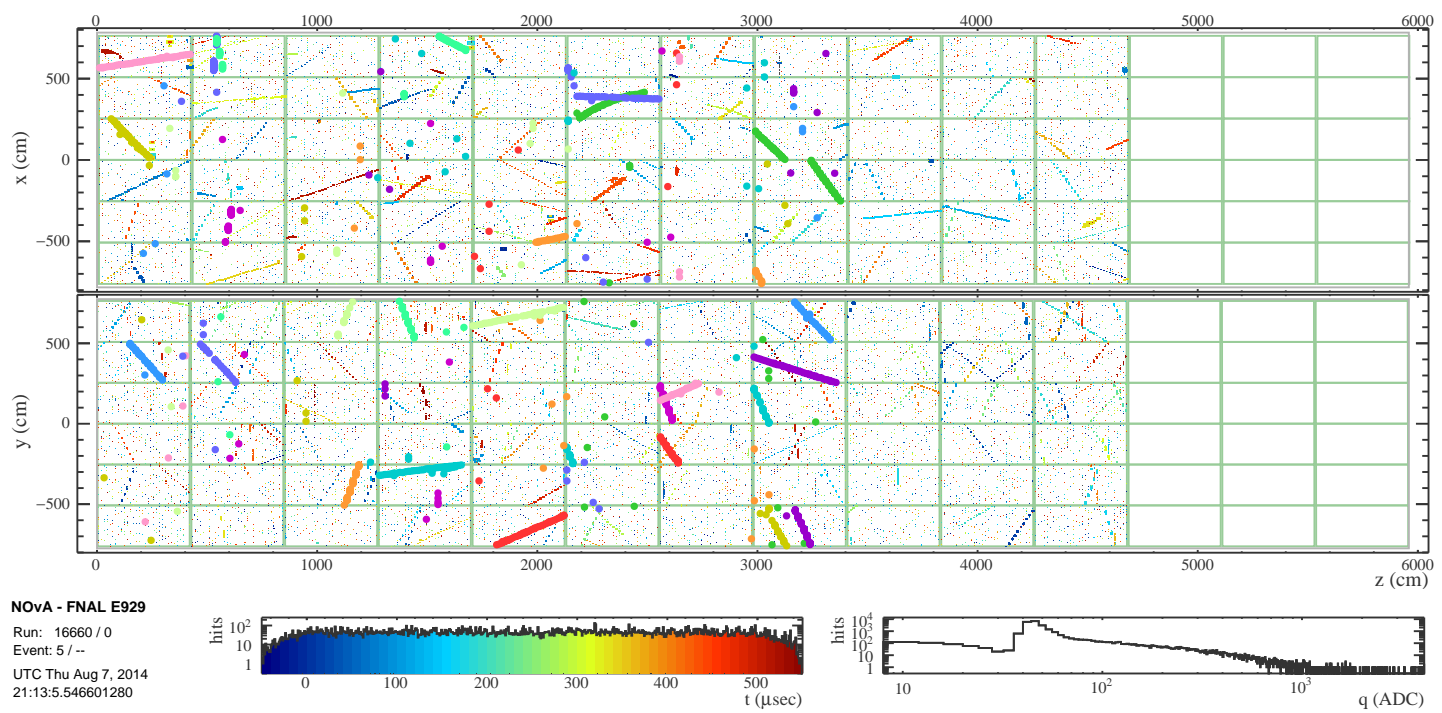

Figure 8.1: A far detector data event where a large number of DCMs are unsynchronized. The green boxes indicate DCM boundaries. Hits are colored by time.

Similar DAQ problems can sometimes cause a large number of DCMs to become unsynchronized with respect to other DCMs. This particular problem is only known to occur in the far detector because of the large number of readout channels, and therefore high data rates that can strain the DAQ system. The signature of such events is that a large number of cosmic tracks appear to terminate on DCM edges, as fig 8.1 shows. The metric designed to reject these events is the fraction of the number of hits on DCM edges that have adjacent hits in adjoining DCM. Hits on detector edges or at the edges of uninstrumented DCMs are not counted towards this ratio.

\subsubsection{Preselection and Cosmic Rejection Cuts}

Preselection and cosmic rejection cuts are applied to every slice and identically to data and Monte Carlo simulation. The cuts, the rationale behind them and their impact are discussed below. 


\section{Skim Cuts}

To speed up the processing time of data and simulation files, we apply two skim cuts during file production: $20<$ number of hits in slice $<200$ and longest prong length $<500 \mathrm{~cm}$. These essentially cut out no $\nu_{e}$ appearance signal, while rejecting a large fraction of cosmic rays and higher energy neutrino interactions that can take a long time to reconstruct. No showers are reconstructed for events that do not pass the skim cuts.

\section{Reconstruction Quality Cuts}

Since LID, the likelihood based $\nu_{e}$ identifier, depends on the reconstruction of vertices and prongs, we require that there be a reconstructed vertex and at least one reconstructed shower, with more than 5 hits in each view, in the slice.

If a large number of channels in a region of the detector are masked out of the analysis or are low in efficiency, the quality of reconstruction in such regions may suffer. A reconstructed prong may get broken into two or more prongs, with the vertex placed either in the middle of the fragments, or at the start of the most upstream prong. In case the vertex is in the middle, one of the prongs is reconstructed as backward going, and we can reject such events by requiring $\cos \theta>-0.95$ between the two showers. If the vertex is at the most upstream end in $z$, the distance of the start of the downstream prong from the vertex is large and can mostly be rejected if we require this distance to be less than $100 \mathrm{~cm}$. Additionally, the asymmetry in the leading shower between the $x$ and $y$ views, defined as $\left|x_{\text {hits }}-y_{\text {hits }}\right| /\left(x_{\text {hits }}+y_{\text {hits }}\right)$ should be less than $40 \%$.

Another slice level feature that needs to rejected is the ringing of APDs after large charge depositions as was discussed in the section 4.3.1. Such slices are characterized by a large number of APDs being nearly fully triggered, ie close to 32 pixels reporting hits. We can reject them efficiently by requiring fewer than 8 hits per plane.

All the cuts listed above are only applied to the far detector. In the near detector, 


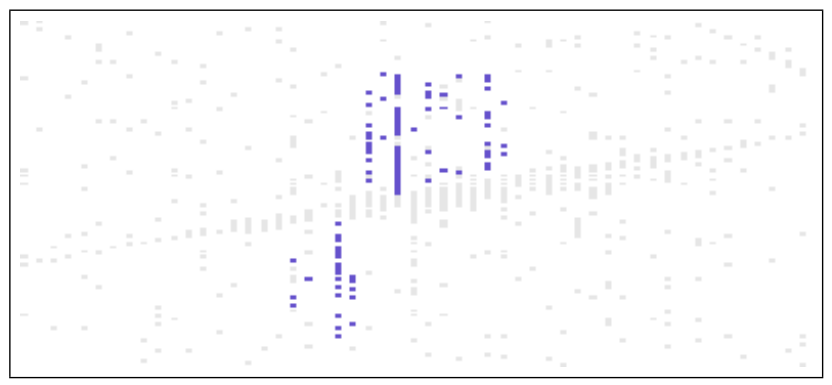

Figure 8.2: Flashing of multiple APDs in an FD data event because of high energy deposits. The gray hits preceded and likely caused the flashing

because of the smaller size of the detector, and nearly 0 cosmic background, we only need simple quality cuts, requiring at least one vertex and one reconstructed shower in the slice, in addition to rejecting APD flashes.

\section{Containment Cuts}

$\mathrm{NO} \nu \mathrm{A}$ is a fully active detector, so any charged particle entering or leaving the detector leaves a track close to the detector edges. Hence containment cuts are not only a way to make sure that the neutrino interacted inside the detector, but they are also a powerful tool in rejecting cosmic ray background.

The far detector dataset contains different diblock configurations, so a statically defined set of containment cuts is not sufficient. In every subrun, we define edges of the detector that are live, ie active in data taking, for that subrun. Shower start and stop points are required to be at least $10 \mathrm{~cm}$ away from the live west and bottom detector faces. Our optimization prefers a slightly tighter cut on the east edge of the detector, requiring a distance greater than $15 \mathrm{~cm}$ to the edge. The east edge is at the far end with respect to the APDs in the horizontal view, so the light has to travel the full length of the cell to produce a hit. Hits due to a cosmic ray entering the east face of the detector may fall below threshold, hence the preference for a tighter cut. The distance from front of the detector is required to be more than $35 \mathrm{~cm}$. 


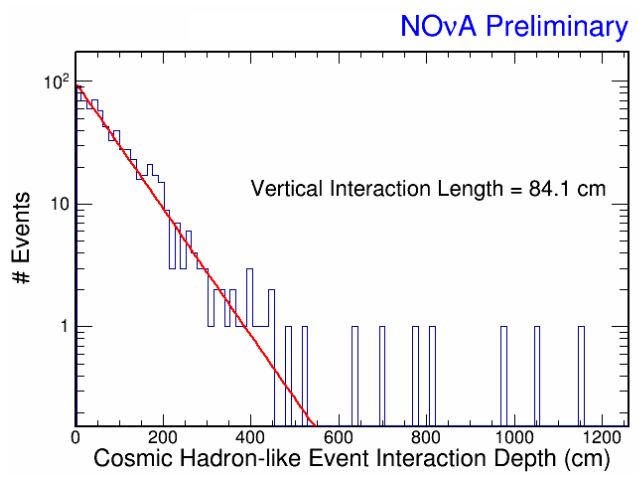

Figure 8.3: Neutron interaction depth in the FD. The data is from the cosmic trigger and the particles were identified as neutron candidates during hand scanning.

Most cosmic rays enter from the top face of the detector. While charged cosmic particles are easy to reject with lenient containment cuts, neutral particles can reach quite deep into the detector before depositing any energy (see figure 8.3). The optimized cut for the minimum distance of the leading shower from the top face is $150 \mathrm{~cm}$. Another tricky background to reject is cosmic ray photons. The overburden on the FD contains barite which is very effective in absorbing photons. At the north end of the detector, the overburden is thin and let's more cosmic photons in. The block-pivoter absorbs photons and helps mitigate the problem somewhat, but as an additional safeguard, the maximum $z$ of the leading shower is required to be more than $200 \mathrm{~cm}$ from the back face. Figure 8.4 shows the distributions of the minimum distance from top and back faces of the detector after preselection and loose PID cuts.

Containment is more straightforward in the near detector because the detector configuration does not change. The containment cuts used in ND are listed in the table below. To cut out activity due to neutrino interactions in the rock preceding the front face of the near near detector, we reject slices with any activity in the first 6 near detector planes. 

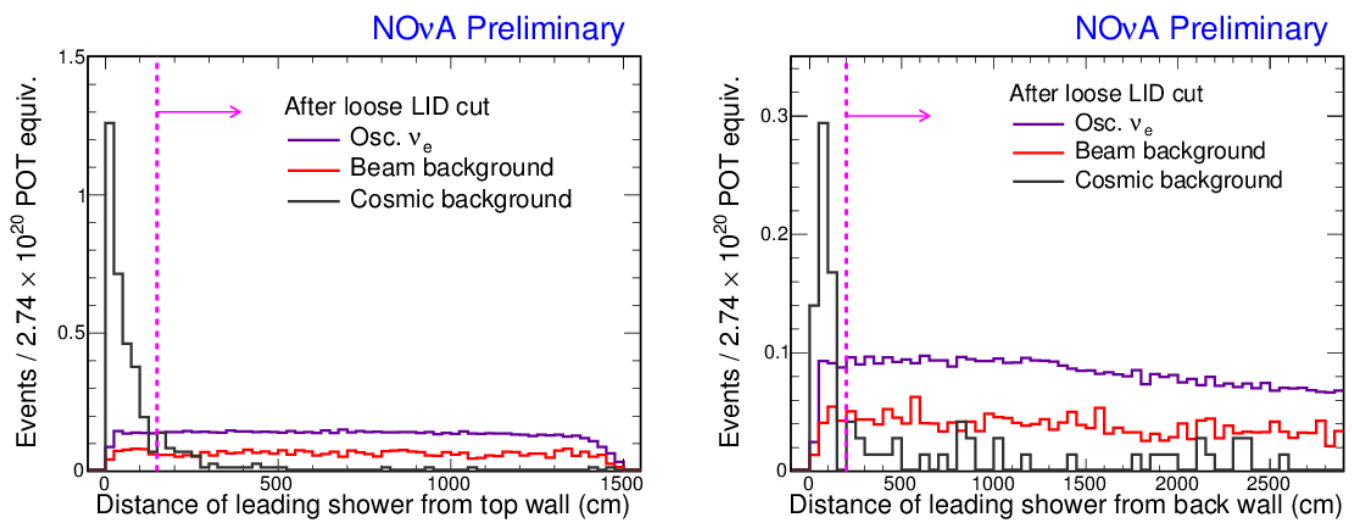

Figure 8.4: Minimum distance of the leading shower in the slice from the top and the back faces of the far detector. Most charged particles in cosmic rays deposit energy close to the boundary of the detector and can be rejected with containment cuts. In these figures, all preselection cuts, along with a loose LID cut of $>0.7$, have been applied except for the $y$ and $z$ containment cuts (respectively).

\section{Additional Cosmic Rejection Cut}

While most cosmic rays are rejected by containment cuts, cosmic neutrons, or those produced by the interaction of cosmic rays in the overburden, are much more difficult to reject, because there is no tell-tale track close to the detector edge. However, cosmic rays tend to align with the azimuth, ie along the $y$ direction in the $\mathrm{NO} \nu \mathrm{A}$ detector coordinate system, while the direction of the NuMI beam in the $\mathrm{NO} \nu \mathrm{A}$ detector coordinates is roughly horizontal, along the $z$ axis. The transverse momentum fraction with respect to the beam direction, which folds in this information, has proved very effective in separation of cosmic rays from neutrino interactions in such cases. This variable also provides some separation of charged current neutrino interactions from neutral currents, because the invisible final state neutrino in NCs can causes an imbalance in the observed transverse momentum. 


\begin{tabular}{l|c}
\hline Variable & Allowed Range $[\mathbf{c m}]$ \\
\hline Vertex X, Y & $(-140,140)$ \\
Vertex Z & $(100,700)$ \\
Shower start, stop X, Y & $(-180,180)$ \\
Shower start, stop Z & $(25,1225)$ \\
\hline
\end{tabular}

Table 8.1: Containment cuts in ND

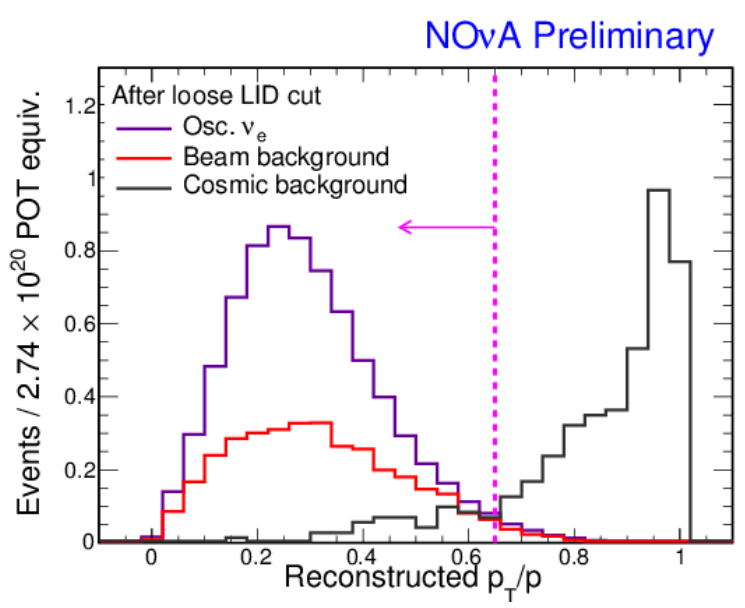

Figure 8.5: Transverse momentum fraction computed with respect to the beam direction, which is nearly horizontal along detector $z$ axis. Cosmics are usually vertical and therefore cluster close to 1 . We cut on this variable at a value of 0.65 . In this plot, all preselection cuts, along with a loose LID cut of $>0.7$, have been applied, other than the cut on $p_{T} / p$

\section{Energy-related Cuts}

NC interactions of high energy neutrinos have low visible energy because of the missing visible energy of the final state neutrino. At $\mathrm{NO} \nu \mathrm{A}$ 's location, the NuMI beam has a narrow peak at $2 \mathrm{GeV}$ and a highly suppressed high-energy tail. This reduces the feeddown from high-energy neutrino NC interactions in the $\nu_{e}$ appearance signal region. 
These interactions, along with cosmic rays, tend to have visible energy less than $1 \mathrm{GeV}$.

The energy cuts on FD events are $1.5 \mathrm{GeV}<E<2.7 \mathrm{GeV}$. Rejecting events with reconstructed energy $>2.7 \mathrm{GeV}$ or number of cells in slice $>115$, cuts out higher energy beam $\nu_{e} \mathrm{CC}$ interactions which otherwise have the same signature as the $\nu_{e}$ appearance signal. Additionally, we require the leading shower in the event to have a length between 140 and $500 \mathrm{~cm}$.

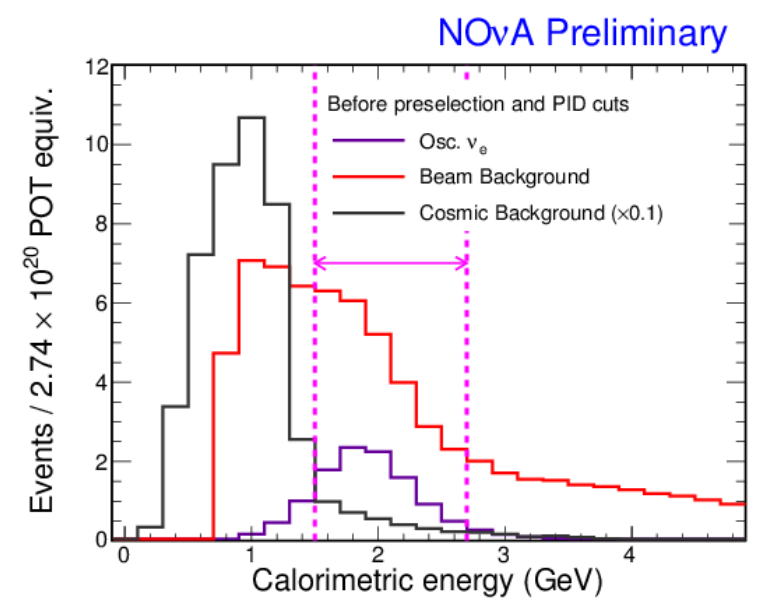

Figure 8.6: Energy distribution with all preselection applied, except for energy cut. The $\nu_{e}$ appearance signal is well-bounded between 1 and $3 \mathrm{GeV}$ while cosmic background clusters at lower energy

In the near detector, energy cuts are looser than in the far detector. The intent is to select events in a wider energy range and extrapolate to FD, because the neutrino energy spectra are similar but not identical for the two detectors.

\subsubsection{Performance of Preselection}

Table 8.3 summarizes the preselection cuts applied in the far detector and table 8.2 shows the performance of these cuts in a sequential manner. The event counts are for real conditions FD MC scaled to $3.516 \times 10^{20}$ POT to reflect the first-analysis expectation, weighted by simple oscillations (see section 5.3). The cosmic background figures are 
from FD cosmic trigger data normalized to the first analysis livetime exposure. The cosmic figures in bracket are raw event count prior to the livetime scaling.

Note that the preselection efficiency changes with the number of diblocks because of the varying fiducial to detector mass ratio, which directly impacts containment efficiency, as figure 8.7 shows. This effect was studied in the real conditions simulation where POTweighted diblock masks from data have been applied to the $\mathrm{MC}$ to imitate the changing detector mass in the first-analysis dataset.

\begin{tabular}{l|ccccl}
\hline Cut & $\nu_{e}$ Signal & $\nu_{\mu} \mathrm{CC}$ & $\mathrm{NC}$ & Beam $\nu_{e} \mathrm{CC}$ & Cosmics \\
\hline After data quality & 20.56 & 314.64 & 15.30 & 211.16 & $5.75 \times 10^{6}\left(4.43 \times 10^{8}\right)$ \\
Reconstruction Quality & 15.18 & 31.05 & 6.10 & 65.73 & $3.55 \times 10^{5}\left(2.74 \times 10^{7}\right)$ \\
Cosmic Rejection & 12.73 & 23.64 & 5.11 & 54.93 & $1.54 \times 10^{4}\left(1.19 \times 10^{6}\right)$ \\
Containment & 11.95 & 21.43 & 4.98 & 45.86 & $307.25(23685)$ \\
Energy-related & 9.16 & 11.32 & 1.12 & 11.52 & $22.09(1703)$ \\
\hline
\end{tabular}

Table 8.2: Event counts on sequential selection before PID cut. The numbers in bracket in the cosmics column are the event counts prior to scaling to the first data livetime.

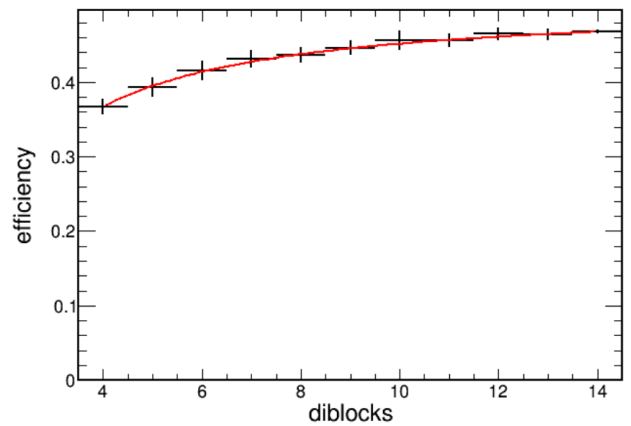

Figure 8.7: Efficiency of $\nu_{e}$ preselection cuts as a function of number of detector diblocks in the FD, as measured in real conditions MC. 


\begin{tabular}{|c|c|}
\hline Cut & Description \\
\hline Skim Cuts & $\begin{array}{l}20<\text { number of hits in slice }<200 \\
\text { Longest prong length }<500 \mathrm{~cm}\end{array}$ \\
\hline Reconstruction quality & $\begin{array}{l}\text { Number of vertices in slice }>0 \\
\text { At least one shower with }>5 \text { hits in each view } \\
\text { If number of showers is } 2, \cos \theta>-0.95 \text { between showers } \\
\text { Vertex to shower start gap }<100 \mathrm{~cm} \\
\text { Shower asymmetry, }\left|x_{\text {hits }}-y_{\text {hits }}\right| /\left(x_{\text {hits }}+y_{\text {hits }}\right)<40 \%\end{array}$ \\
\hline Containment & $\begin{array}{l}\text { Dist. of leading shower start and stop to west face }<10 \mathrm{~cm} \\
\text { Dist. of leading shower start and stop to bottom face }<10 \mathrm{~cm} \\
\text { Dist. of leading shower start and stop to east face }<15 \mathrm{~cm} \\
\text { Dist. of leading shower start and stop to front face }<35 \mathrm{~cm} \\
\text { Dist. of leading shower start and stop to top face }<150 \mathrm{~cm} \\
\text { Dist. of leading shower start and stop to back face }<200 \mathrm{~cm}\end{array}$ \\
\hline Cosmic rejection & Transverse momentum fraction $p_{T} / p<0.65$ \\
\hline Energy-related cuts & $\begin{array}{l}20<\text { number of hits in slice }<115 \\
140 \mathrm{~cm}<\text { Longest prong length }<500 \mathrm{~cm} \\
1.5 \mathrm{GeV}<\text { Event energy }<2.7 \mathrm{GeV}\end{array}$ \\
\hline
\end{tabular}

Table 8.3: Summary of $\nu_{e}$ preselection cuts applied in the far detector

It is clear that preselection does not get us a pure enough sample of $\nu_{e}$ CC signal events for the appearance analysis. The final signal to background separation is done by the particle identification algorithms, as we will discuss next. 


\section{2 $\nu_{e}$ Particle Identification}

We have two main approaches to identifying $\nu_{e}$ appearance signal. The Likelihood-based particle ID, or $L I D$, in particular looks for an electron-induced electromagnetic shower in the event. This section describes LID method of $\nu_{e}$ identification in detail.

An alternative approach to LID has been implemented in the form of Library Event Matching, or $L E M$. It consists of a vast library of simulated $\nu_{e}$ signal and background interactions. A candidate event is compared against all library events to identify the class of events that the candidate is most akin to. The properties of the 1000 best matches found from the library are fed into an ensemble of decision trees to obtain a PID value. This method is reported in detail in [42].

\subsubsection{Likelihood-based Particle Identification}

The energy deposited per unit length, $d E / d x$, by a particle, along its trajectory is a characteristic of the particle. LID uses this information to compute the likelihood that the candidate particle is an electron. These likelihood variables, along with other topological information about the event are used as input to an Artificial Neural Network or ANN to construct a particle ID. The steps involved in achieving that end are described below.

\section{Fine-tuning the reconstruction}

LID uses prongs made by the Fuzzy-K algorithm, but these prongs are not reconstructed or shaped to fit any particular particle hypothesis. LID performs some reclustering of the Fuzzy-K prongs to conform to the shape expected of an electron shower. A typical prong due to an electron is narrow close to the vertex, where the electron is minimum ionizing and has not yet begun to radiate. Once a brehmsstrahlung photon is emitted, the shower commences and the prong broadens. The reclustering in LID labels all hits within a radius of 2.5 cell widths of the shower core in the first 8 planes and 20 cell 
widths for the rest of the planes as belonging to the shower. The smaller radius at the start of the shower also reduces contamination of the shower by other hadronic activity close to the vertex.

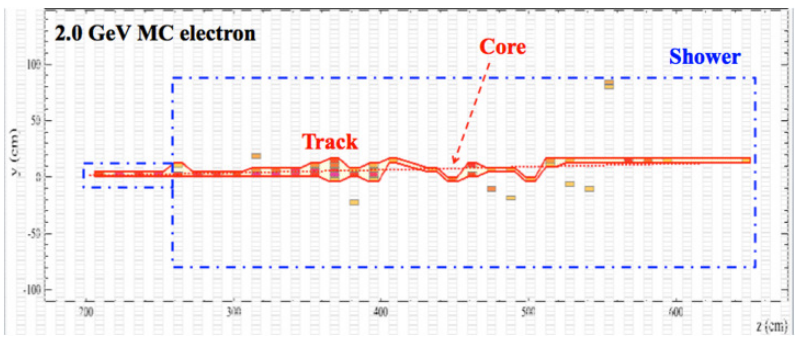

Figure 8.8: Fuzzy-K prongs are reclustered to better fit the electron shower shape.

As discussed before, Fuzzy-K allows hits to be associated with multiple prongs. LID performs cell-energy deconvolution to ensure that a cell's energy is not double counted towards the event energy. For a cell common to multiple shower, its energy contribution to the $i$ th shower is set to:

$$
E_{i}^{\text {cell }}=\frac{P E^{\text {cell }}}{a_{i}} \cdot \frac{P E_{i}^{\text {shower }} \times e^{-D_{i} / \lambda}}{\sum_{j} P E_{j}^{\text {shower }} e^{-D_{j} / \lambda}}
$$

\section{NOvA Monte Carlo}

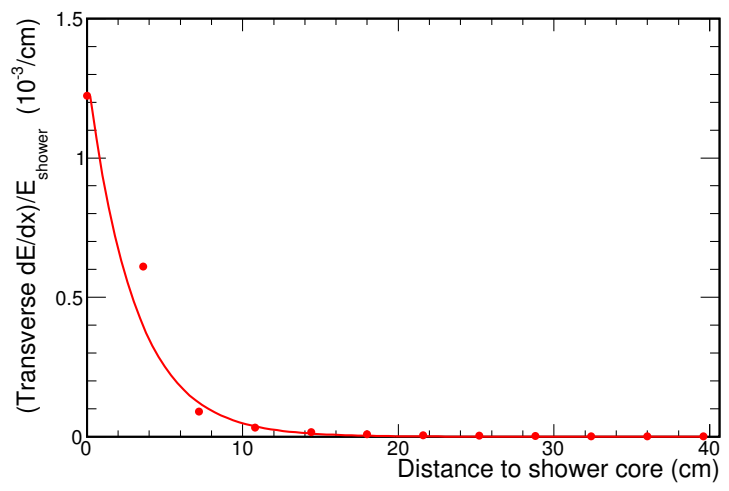

Figure 8.9: Transverse $d E / d x$ per electron energy as a function of distance from the shower core. The distribution is fit with 8.2 
Here, $P E^{\text {cell }}$ is the number of photo-electrons in that cell and $P E^{\text {shower }}$ is the total $\mathrm{PE}$ in the $i$ th shower. $a_{i}$ is the attenuation calibration factor to convert PE to GeV, based on the average distance of the $i$ th shower from the readout. $D_{i}$ is the distance of the hit from the $i$ th shower core and $\lambda$ is a constant that describes the shower lateral profile in the medium, ie the transverse $d E / d x$ as a function of distance from the shower core is

$$
\frac{d E}{d x}(w)=A E e^{-w / \lambda}
$$

where $E$ is the shower energy, $A$ is a normalization constant and $w$ the distance from the shower core. Based on simulated electron showers, $\lambda$ is determined to be $3.05 \mathrm{~cm}$, as shown in fig.8.9.

\section{Particle Likelihood}

After reclustering and energy deconvolution, the most energetic shower in the event is picked for identification. The particle hypothesis that LID tests the leading shower against are $e, \mu, p, n, \pi^{ \pm}, \pi^{0}$ and $\gamma$. To construct likelihoods, the $d E / d x$ information is recorded in longitudinal and transverse views. The longitudinal view is along the length of the shower and transverse view is perpendicular to the direction of the shower and summed over all planes in the shower.

In the longitudinal view, the $d E / d x$ is recorded for every detector plane that the particle passes through. The transverse view is a measure of how concentrated the energy deposition is in the core of the shower. Therefore, the $d E / d x$ is recorded radially, as a function of the number of cell-widths from the core of the shower. Figure 8.10 shows an example of how different particles differ in $d E / d x$.

Template histograms of the expected plane-by-plane $d E / d x$ and transverse view depositions are created for all the particle hypotheses using simulated neutrino interactions. These histograms are normalized to form probability distribution functions. The energy depositions of a prong of unknown identity are compared against these template histograms. For instance, if an unknown particle deposits energy $E_{i}$ in the $i$ th plane 

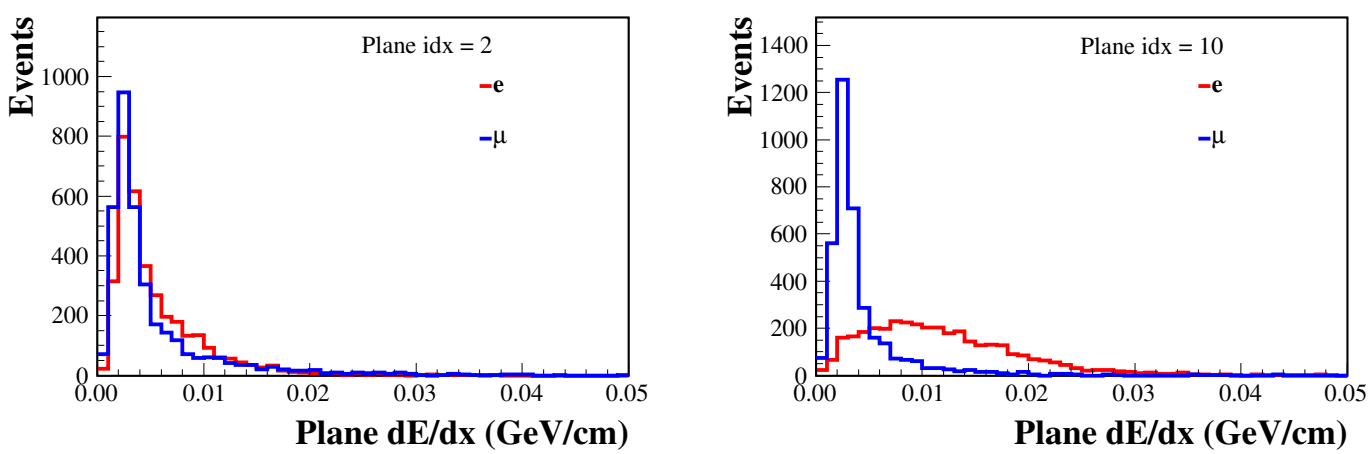

Figure 8.10: Muon and electron $d E / d x$ in the second and tenth plane from the shower start. In most cases, by the second plane, the electron has begin to shower and behaves as a minimum ionizing particle, just as the muon does. By the tenth plane, it is very likely that the EM shower has begun to develop.

from the start of the prong, the probability, $P_{i}$ of various particle hypotheses depositing energy in this manner is read from the template histograms for the $i$ th plane. The logarithms of these probabilities are then summed over all planes in the prong and used to construct a likelihood for that particle hypothesis:

$$
L L\left(e^{-}\right)=\sum_{i}^{N} \log \left(P_{i}\left(e^{-}\right)\right) / N
$$

where $L L\left(e^{-}\right)$is the likelihood of the unknown particle being an electron. $N$ is the total number of planes in case of longitudinal likelihood and total number of transverse cells in transverse likelihood. Likelihood differences are found to be more useful quantities since they are relative probabilities. So the likelihood difference $L L\left(e^{-}\right)-L L\left(\mu^{-}\right)$would be clustered towards higher values for electron showers and towards lower values for true muon tracks. Figure 8.11 shows the discriminating power of these likelihood ratios for simulated electron, muons and neutral pions. 

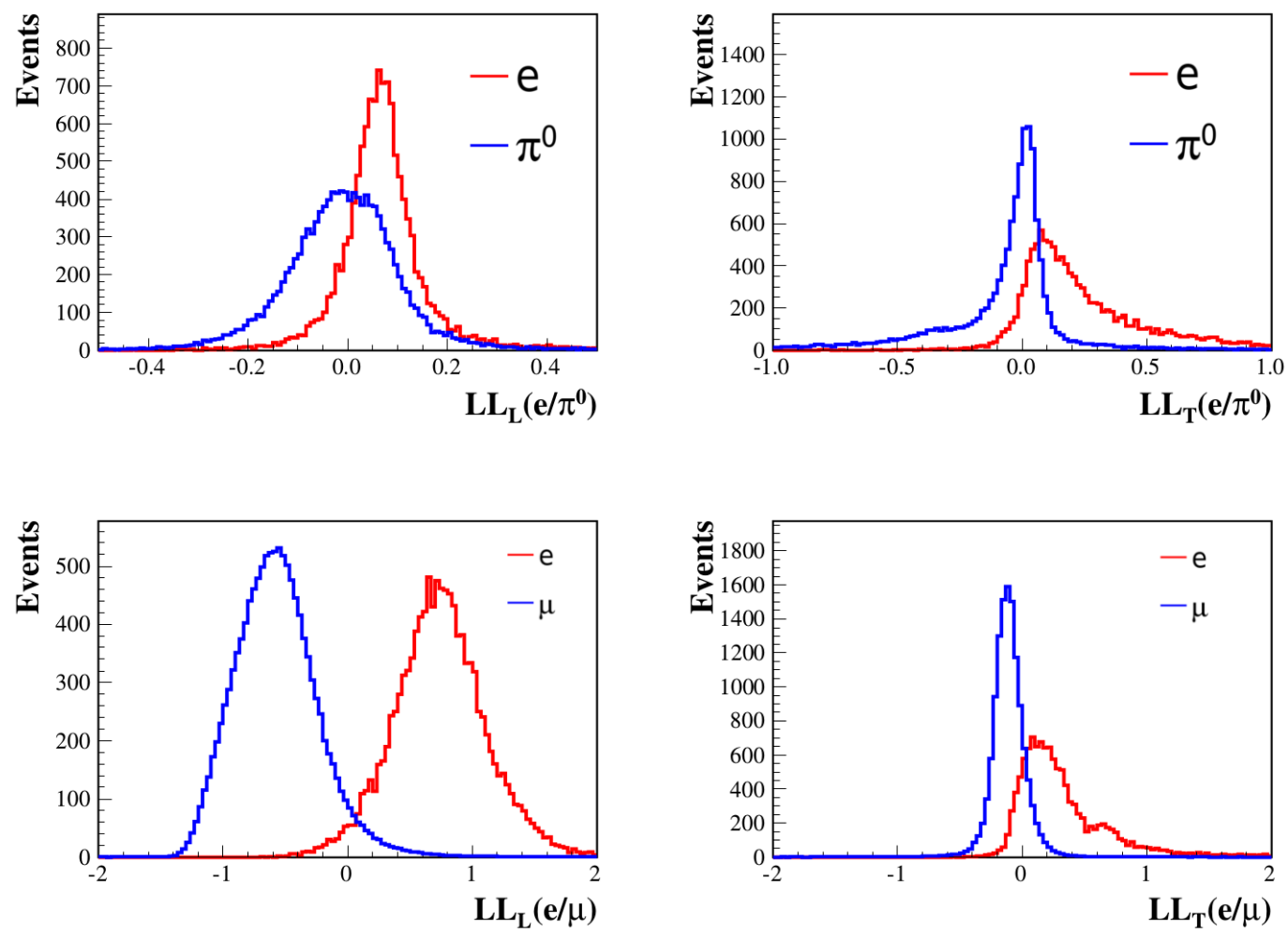

Figure 8.11: Longitudinal and transverse likelihood difference $L L\left(e^{-}\right)-L L\left(\pi^{0}\right)$ for electrons and neutral pions, and $L L\left(e^{-}\right)-L L(\mu)$ for electrons and muons. While the lateral profile of $\pi^{0}$ and electrons do not show much separation, the transverse likelihood ratio is better separated. This is because $\pi^{0} \mathrm{EM}$ showers often contain two overlapping decay photons. Muons and electrons are well-separated in both views.

\section{The Neural Net}

LID uses ROOT's TMultiLayerPerceptron class which is an implementation of a feedforward neural network. Neural networks are a computational method of approximating a non-linear functional mapping between input variables and an output. The network is presented with input variables for a training sample for which the signal and background populations are known, and it learns what combinations of inputs maps to signal and 
to background.

A neural network consists of an input an output layer of neurons and one or more hidden layers where the computation takes place. The number of neurons in the input layers corresponds to the number of input variables to the network. It passes on the input to the first hidden layer. The neurons in hidden layers are characterized by a bias and weighted connections to neurons in the next layer, called synapses. Each neuron in a hidden layer receives a linear combination of the output of neurons from the previous layers, and makes independent calculations. Each has a pre-defined activation function, which it uses to compute its output, based on the inputs it received. The activation functions are usually sigmoids. For each training event, the network compares the output to the correct answer and readjusts the neuron biases to reduce the error.

\section{Additional LID Variables}

The goal of $\nu_{e}$ particle identification is to select $\nu_{e} \mathrm{CC}$ appearance signal with high efficiency and purity. The $\nu_{e}$ appearance signal is expected to peak between 1 and 3 $\mathrm{GeV}$.

The presence of an obvious muon track makes $\nu_{\mu} \mathrm{CC}$ interactions relatively easy to separate from the signal and the likelihood variables are very efficient at doing so. $\nu_{\mu}$ CC's may be a background when the interaction is highly inelastic (high hadronic- $y$ ) and the hadronic shower contains an EM-like shower, though the rate of this background is sufficiently small.

Neutral currents are more difficult to separate, since there is no muon track for easy separation, and these interactions often produce neutral pions whose decay $\left(\pi^{0} \rightarrow \gamma \gamma\right)$ produces electromagnetic showers that are a potential background. Showers induced by photons are different from those produced by electrons, since electrons being charged, deposit energy right away. The photon may travel some distance before converting and

producing a shower. LID uses a reconstructed $\pi^{0}$ invariant mass and the distance of the shower start to the event vertex to help reject this background. Moreover, neutral 
currents have a neutrino in the final state which is not visible. So the angular distribution of EM-like showers in NC's is also different. We use $\cos \theta$, the angle of the leading shower with respect to the beam direction, as a variable in LID to exploit this properly. This variable is also useful for cosmic background rejection in FD since cosmics are mostly vertical. Additionally, NC interactions that are a background to the appearance signal are usually highly inelastic and have a smaller fraction of event energy contained in the leading shower. This too is an input variable to the ANN.
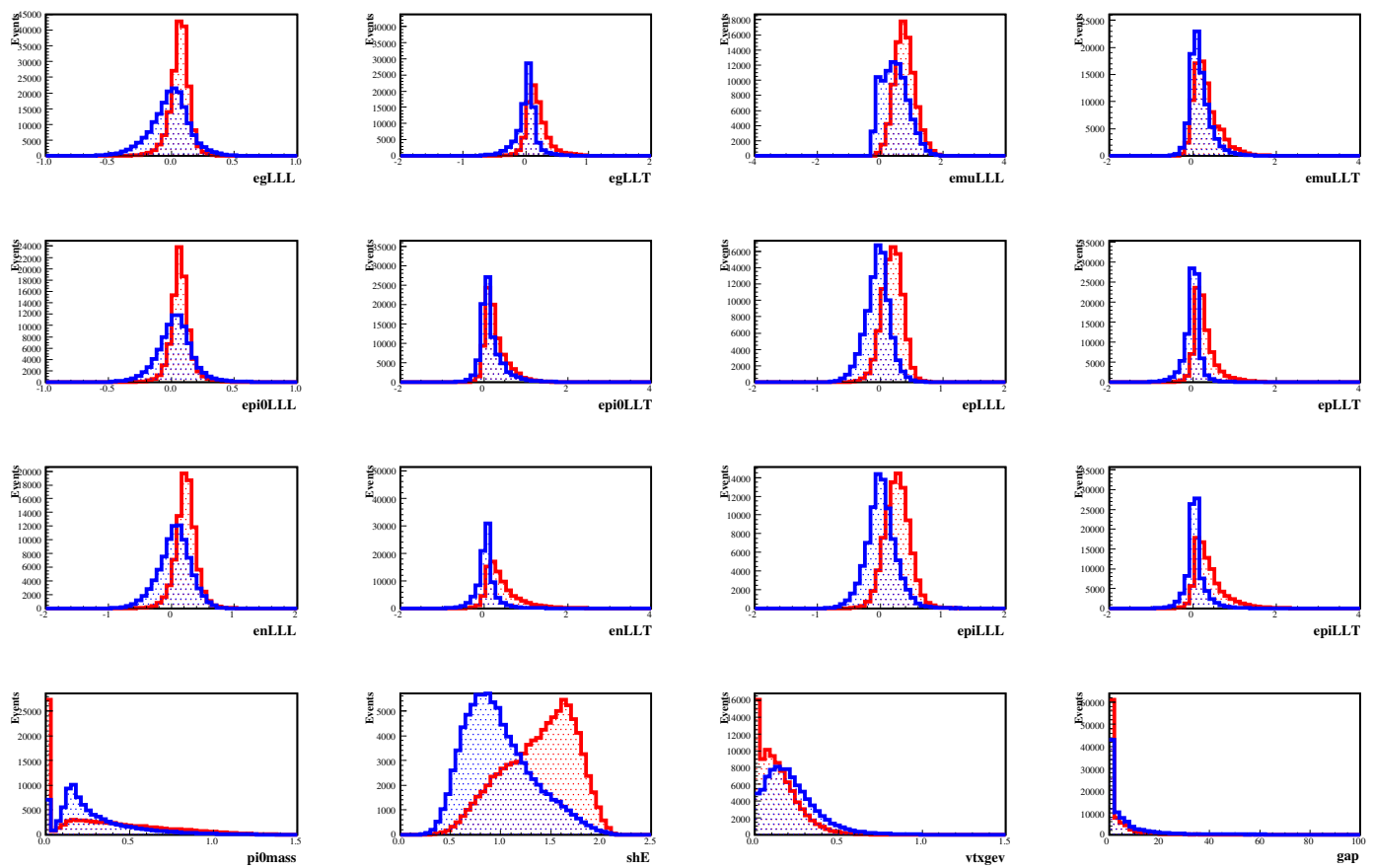

Figure 8.12: Distribution of ANN input variables for signal(red) and background (blue)

The intrinsic beam $\nu_{e}$ component is an irreducible background since these interactions are identical to the $\nu_{e}$ appearance signal. The only handle that we have to reject the intrinsic $\nu_{e}$ component is that the energy distribution of the two samples is differentthe $\nu_{e}$ appearance signal is a narrow peak around $2 \mathrm{GeV}$, while the beam $\nu_{e}$ 's are a much more spread out higher energy distribution. The energy cuts discussed earlier in the 
chapter are efficient at reducing this 'background'.

To summarize, in addition to the particle likelihoods, LID uses the following additional input variables for identification:

1. Shower energy fraction: shower energy divided by the total event energy.

2. $\pi^{0}$ mass: The invariant mass of the most energetic shower with all the other showers in the slice is computed and the one closest to the $\pi^{0}$ mass is saved

3. Vertex energy: The calorimetric energy within \pm 8 planes from the event vertex

4. Gap: The distance of the start point of the shower from the event vertex.

5. $\cos \theta$ : Angle of the leading shower with respect to the beam direction

The cut on PID value has been optimized to maximize two statistical figures of merit $(\mathrm{FoM})$. The FoM $s / \sqrt{b}$ is appropriate for a $\nu_{e}$ appearance search on a large background, and the PID value tuned to maximize it gives a value of 0.95 . Optimizing to maximize $s / \sqrt{(s+b)}$ gives a value of 0.61. The PID shape and selected event counts are given in figure 8.13 and table 8.4. A combination of preselection and PID cuts reduces the enormous cosmic background from $5.75 \times 10^{6}\left(\left(4.43 \times 10^{8}\right)\right.$ events to $0.04(3)$ events for the livetime equivalent to the first data exposure. As before, the figures in the bracket are the counts before scaling. The beam backgrounds continue to be the bigger problem and work aiming towards future $\mathrm{NO} \nu \mathrm{A}$ analyses is under way to polish our selections and improve beam background rejection efficiency.

\subsection{Final Cosmic Background Prediction}

The $\nu_{e}$ selection and cosmic rejection cuts presented thus far have been tuned on a combination of cosmic trigger data and the oscillated NuMI beam simulation. Once the cuts were tested and found to perform optimally, they were applied to the NuMI out-of-time sideband to get the final estimate of cosmic background to the appearance 


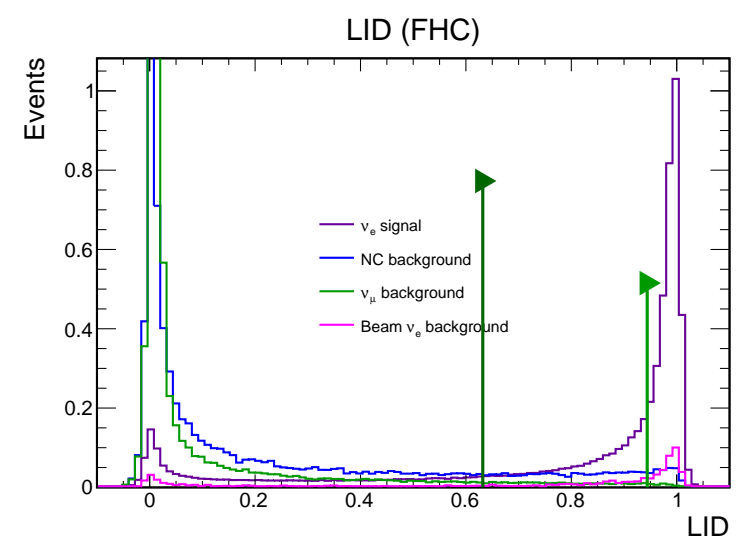

Figure 8.13: LID distribution for $\nu_{e}$ appearance signal and beam backgrounds. The distributions are weighted by oscillation probability with no matter effect and scaled to the expected event count for $1.9 \times 10^{20}$ Protons on Target (POT). The two arrows indicate the cuts that maximize the figure of merit $s / \sqrt{b}$ (light green) and $s / \sqrt{s+b}$ (dark green)
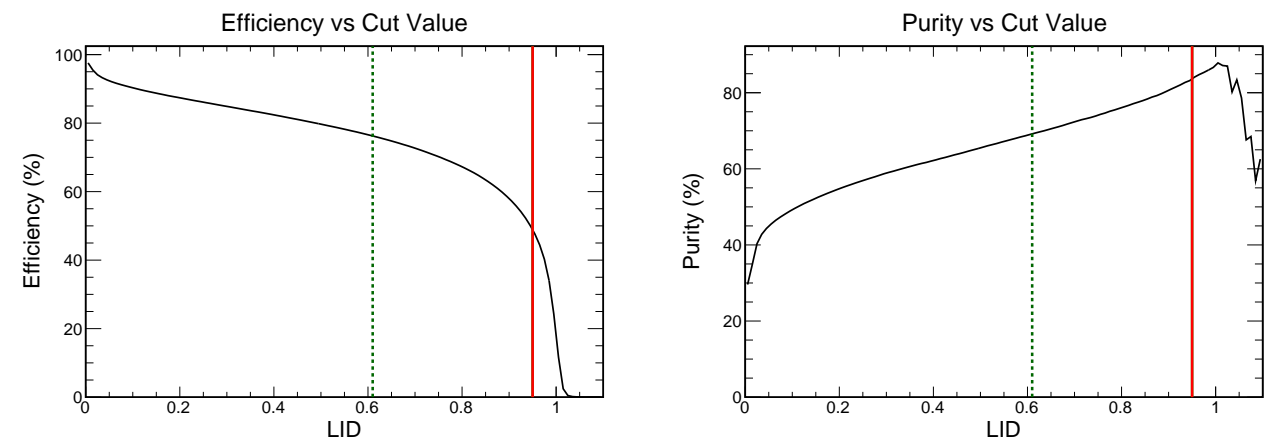

Figure 8.14: Efficiency of LID selection and purity of selected sample as a function of LID cut. The dotted green line is the cut optimized for maximum $s / \sqrt{s+b}$ and and the red for maximum $s / \sqrt{b}$. The efficiency is measured with respect to preselection.

analysis of first data. The event counts of cosmics in NuMI sideband are presented in table 8.5, along with those in cosmic trigger data for reference. 


\begin{tabular}{l|l|l|l|lllll}
\hline & Cut & $\nu_{e}$ sig & Tot bg & NC & $\nu_{\mu}$ CC & Beam $\nu_{e}$ & Sig eff & Purity \\
\hline$\frac{s}{\sqrt{b}}$ opt & 0.95 & 4.36 & 0.82 & 0.33 & 0.05 & 0.44 & $47.57 \%$ & $84.10 \%$ \\
$\frac{s}{\sqrt{s+b} \text { opt }}$ & 0.61 & 6.97 & 3.08 & 1.82 & 0.47 & 0.79 & $76.08 \%$ & $69.36 \%$ \\
\hline
\end{tabular}

Table 8.4: Performance of LID for cut optimized to maximize the figure of merit in two different ways. All selection cuts discussed in section 8.1 have been applied. Signal efficiency is measured with respect to the preselected sample. Event counts are normalized to first data exposure

\begin{tabular}{l|ll}
\hline Cut & Cosmic Trigger & NuMI Out-of-time Sideband \\
\hline After data quality & $5.75 \times 10^{6}\left(4.43 \times 10^{8}\right)$ & $5.86 \times 10^{6}\left(1.59 \times 10^{8}\right)$ \\
Reconstruction quality & $3.55 \times 10^{5}\left(2.74 \times 10^{7}\right)$ & $3.62 \times 10^{5}\left(1.00 \times 10^{7}\right)$ \\
Containment & $1.54 \times 10^{4}\left(1.19 \times 10^{6}\right)$ & $1.56 \times 10^{4}\left(4.40 \times 10^{5}\right)$ \\
Cosmic Rejection & $307.25(23685)$ & $312.16(8403)$ \\
Energy-related Cuts & $22.09(1703)$ & $23.24(642)$ \\
LID & $0.04(3)$ & $0.06(2)$ \\
\hline
\end{tabular}

Table 8.5: Cut flow on cosmic ray background in out-of-time NuMI data. Event counts in bracket are prior to scaling the livetime to first data.

To summarize, in the NuMI out-of-time sideband, only two cosmic ray events survive all $\nu_{e}$ selection. Scaling to the total NuMI spill livetime in the first data, this results in a cosmic background estimate of 0.06 events for this analysis, a background negligible in comparison to other beam neutrino backgrounds. This finding is consistent with the background count obtained from the cosmic trigger data at the $0.41 \sigma$ level. 


\section{Summary}

In the $\nu_{e}$ analysis, we use a combination of simple, well-motivated cuts to control data quality and to reject some of the backgrounds. The fully active nature of the detector allows the use of detector edges as a veto region and makes cosmic rejection a manageable task. The angular separation of beam from cosmics is also found to be a useful variable. Other topological variables such as prong length and event energy help in rejecting some of the beam backgrounds too.

We rely on more complex neural-network based techniques to separate the appearance signal from harder to separate backgrounds. We are able to select $\nu_{e} \mathrm{CC}$ appearance signal with an efficiency of $47.57 \%$ (with respect to preselected sample) and achieve a signal purity of $84.1 \%$. The cosmic background is also reduced to a negligible level, where we achieve a suppression of 95.8 million to 1 . 


\section{Chapter 9}

\section{Near Detector Data}

The near detector (ND) is a $0.3 \mathrm{kT}$ version of the $\mathrm{NO} \nu \mathrm{A}$ far detector located $1 \mathrm{~km}$ from the neutrino source at Fermilab. Being located this close to the source, the ND records multiple neutrinos per beam spill and the ND data comprise a high statistics sample of neutrino interactions. They helps us understand how our detectors respond to events that we are most interested in and how well we simulate them. This chapter presents the studies that have been conducted thus far to comprehend the ND data and some discussion on how the data might be interpreted for future analyses.

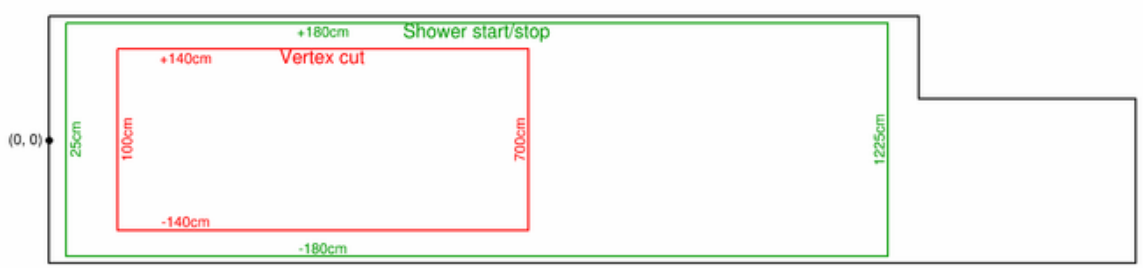

Figure 9.1: Near detector fiducial and containment bounds for $\nu_{e}$ selection 


\begin{tabular}{|l|l|}
\hline Cut name & Value \\
\hline Data quality & Hits per plane $\geq 8$ (reject APD flash) \\
Reconstruction Quality & Number of vertices, 3D prongs, shower $>0$ \\
Fiducial cut & $\mid$ Vertex $x, y \mid<140 \mathrm{~cm}, 100 \mathrm{~cm}<$ Vertex $z<700 \mathrm{~cm}$ \\
Containment cut & $\mid$ shower start, stop $x, y \mid<180 \mathrm{~cm}, 25 \mathrm{~cm}<$ shower start stop $z<1225 \mathrm{~cm}$ \\
Front Planes & Minimum distance of slice to front face $>6$ planes \\
Slice hits and $E_{\text {cal }}$ & $20<$ hits in slice $<200, E_{\text {cal }}<5$ \\
Shower length & $140 \mathrm{~cm}<$ longest prong length $<500 \mathrm{~cm}$ \\
Gap & Leading shower start distance to vertex $<100 \mathrm{~cm}$ \\
$\nu_{e}$ PID & LID $>0.95$ \\
\hline
\end{tabular}

Table 9.1: Near detector $\nu_{e}$ selection cuts

\subsection{Near Detector Data-MC Comparisons}

It was discussed earlier (chapter 6) that studies of the near detector data prompted the incorporation of APD sag and Birks-Chou suppression into our simulation, which went a long way in improving data-MC agreement in the ND. All those refinements are included in the simulation that is compared with the data in this section.

\subsubsection{ND $\nu_{e}$ Event Selection}

The selection cuts applied in the near detector have previously been considered in chapter 8; nonetheless they are reviewed here in table 9.1. Figure 9.1 is a diagram of the ND with event containment boundaries shown. The selection efficiencies for each cut level are in table 9.2. The event counts are normalized by POT equivalent to the first ND data exposure of $1.66 \times 10^{20}$ POT.

Prior to any cuts, MC has only a $0.8 \%$ excess over data, so the integrated flux $\times$ cross-section simulation is remarkably good. After all preselection cuts are applied, however, this excess increases to $5.9 \%$, while after applying the final PID cut, it is the data that have a $3 \%$ excess over MC. According to the simulation, the final $\nu_{e}$ selected events in the ND comprise $16 \% \nu_{\mu} \mathrm{CC}, 52.3 \%$ beam $\nu_{e} \mathrm{CC}$ and $31.7 \% \mathrm{NC}$ interactions.

Figures 9.2, 9.3 and 9.4 show distributions of some key variables after all cuts listed 


\begin{tabular}{|c|c|c|c|c|c|c|c|c|c|}
\hline & Total MC & Efficienc & & $\nu_{\mu} \mathrm{CC}$ & $\nu_{e} \mathrm{CC}$ & $\mathrm{NC}$ & Data & Efficiency & \\
\hline No cut & 30049057 & $100.00 \%$ & & 25742957 & 364671 & 3941430 & 29802297 & $100.00 \%$ & \\
\hline Data quality & 29139394 & $96.97 \%$ & & 25132969 & 339183 & 3667242 & 29015588 & $97.36 \%$ & \\
\hline Reconstruction & 16338569 & $54.37 \%$ & & 13577267 & 229803 & 2531499 & 15996841 & $53.68 \%$ & \\
\hline Fiducial & 1139793 & $3.79 \%$ & & 758778 & 20924 & 360091 & 1025407 & $3.44 \%$ & \\
\hline Containment & 478835 & $1.59 \%$ & & 253650 & 10236 & 214949 & 424518 & $1.42 \%$ & \\
\hline Front planes & 461975 & $1.54 \%$ & & 243569 & 9815 & 208592 & 413242 & $1.39 \%$ & \\
\hline Slice hits and $E_{\text {cal }}$ & 323131 & $1.08 \%$ & & 177970 & 5437 & 139724 & 301019 & $1.01 \%$ & \\
\hline Shower length & 236834 & $0.79 \%$ & & 145944 & 4859 & 86030 & 222046 & $0.75 \%$ & \\
\hline Gap & 230475 & $0.77 \%$ & $100.00 \%$ & 142987 & 4755 & 82732 & 217656 & $0.73 \%$ & $100.00 \%$ \\
\hline LID & 2471 & $0.01 \%$ & $1.07 \%$ & 396 & 1292 & 783 & 2579 & $0.01 \%$ & $1.18 \%$ \\
\hline
\end{tabular}

Table 9.2: $\nu_{e}$ event selection efficiency in Near Detector for simulation, split by interaction type and data.

in table 9.1 except for PID are applied. The preselected events are dominated by $\nu_{\mu}$ CC interactions (62\%). Figure 9.2a shows the reconstructed energy of the interactions; the data peak lower than the simulation by about $15 \%$. The energy per hit in slice and energy of the leading shower (figures $9.2 \mathrm{~b}$ and $9.3 \mathrm{a}$, respectively) are also biased low in data, but the degree of disagreement is smaller. It is clear from figure $9.3 \mathrm{~b}$ that most of the disagreement in the event energy between data and MC arises from the poorly modeled hadronic energy in simulation. The hadronic energy is defined here as all the energy in the event that is not on the leading shower. Since no $\nu_{e}$ or $\nu_{\mu}$ PID cut has been applied at this stage, the leading shower or track is not guaranteed to be an electron or a muon. So the hadronic energy after preselection may have some non-hadronic component.

Further, figure 9.3c shows a large disagreement in the number of reconstructed showers or the observed multiplicity in the events. The electron-muon log-likelihood difference in figure $9.3 \mathrm{~d}$ has two distributions. The peak to the left, at negative values of the difference, is dominated by $\nu_{\mu} \mathrm{CC}$ interactions where the leading shower is the muon, and therefore, very unlike the electron. The one on the right consists of neutral currents and $\nu_{\mu} \mathrm{CC}$ interactions where a hadron is the primary shower. The beam $\nu_{e}$ 
$\mathrm{CC}$ populate the region further to the right, at higher values of the difference. From this distribution too it appears that hadronic component of neutrino interactions is not modeled properly in our simulation. Such modeling problems have a direct impact on the behavior of higher level variables such as the PID which is shown in figures 9.4a and 9.4b. LID shows an excess in data in the signal region (LID > 0.95), but everywhere else, the data are $5-10 \%$ lower than MC.

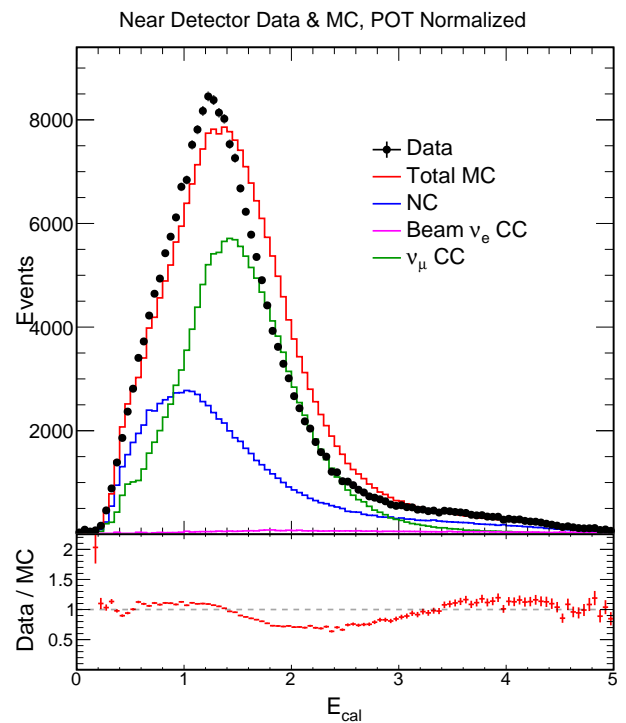

(a) Reconstructed neutrino energy

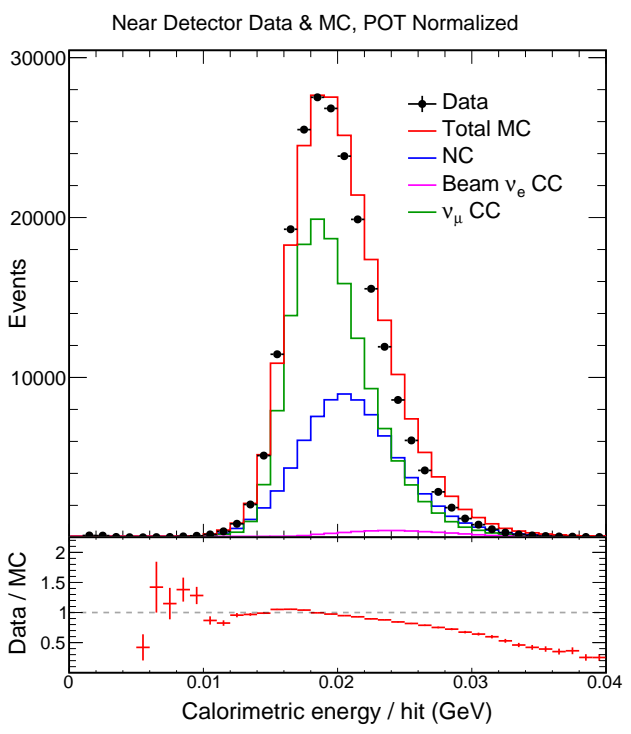

(b) Energy per hit in slice

Figure 9.2: Data and MC comparison of ND $\nu_{e}$ preselected events. 

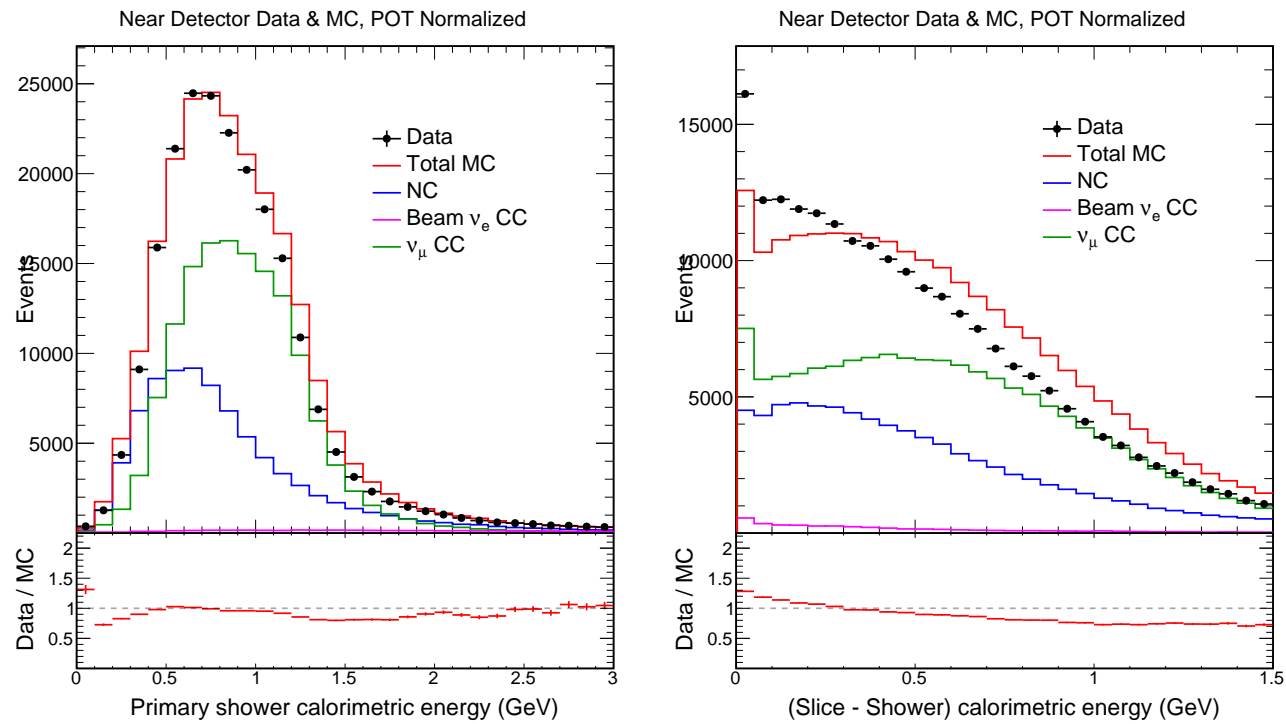

(a) Reconstructed energy of the leading (b) Energy in slice that is not on the leading shower in the event shower, or the hadronic energy in the event.
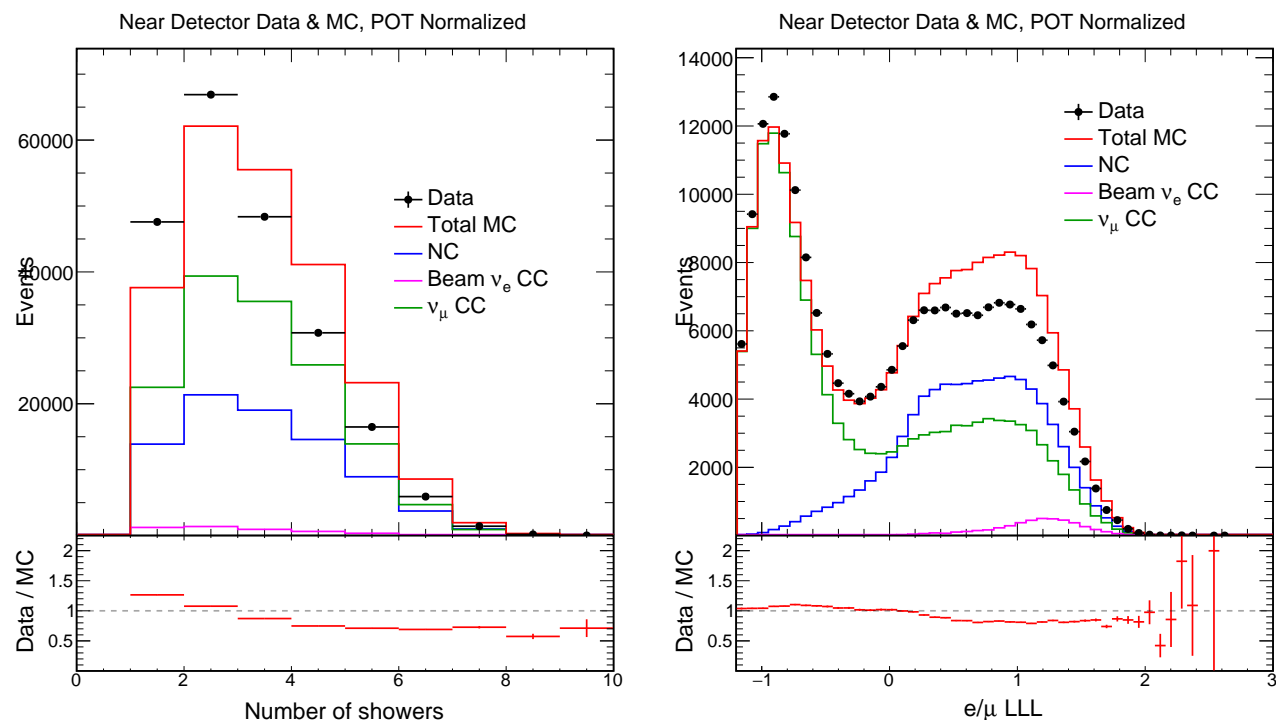

(c) Number of reconstructed 3D showers in (d) Electron-muon longitudinal logevent.

likelihood difference

Figure 9.3: Data and MC comparison of ND $\nu_{e}$ preselected events. The MC is broken down by interaction type. 


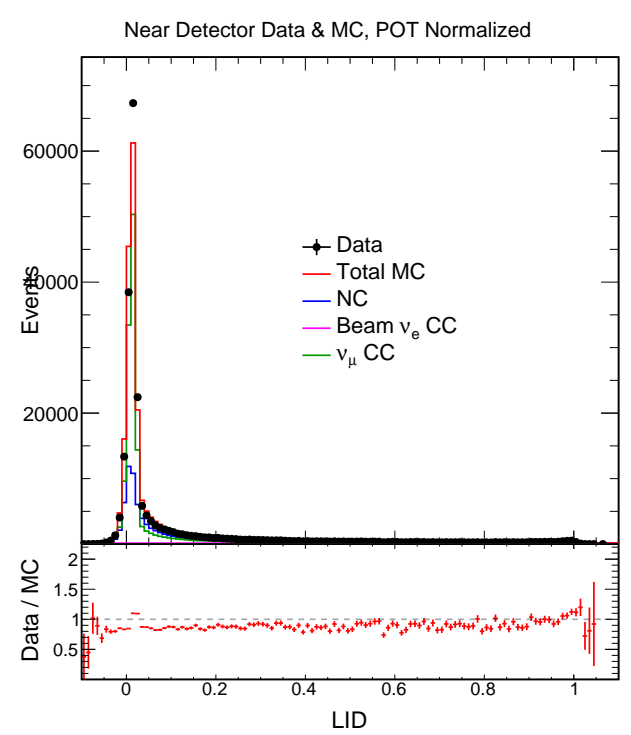

(a) LID

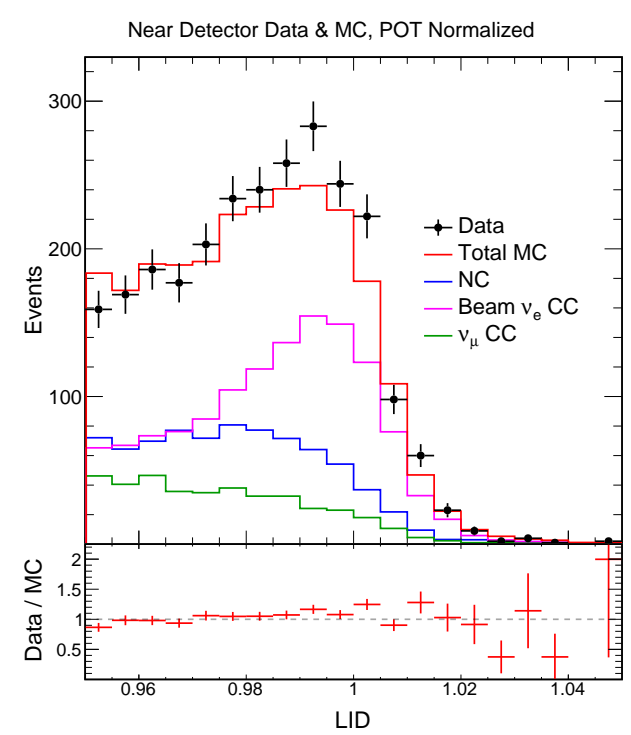

(b) LID zoomed in on the signal region

Figure 9.4: Data and MC comparison of ND $\nu_{e}$ preselected events.

The distributions in figures 9.2 and 9.3 are repeated in figures 9.5 and 9.6 after applying the PID cut of LID $>0.95$. The data-MC agreement is much better once the PID cut is applied and it is ensured that there is some electromagnetic activity in the event. The hadronic energy in the interaction and the event multiplicity, however, continue to be discrepant between data and MC (figure 9.5d). 


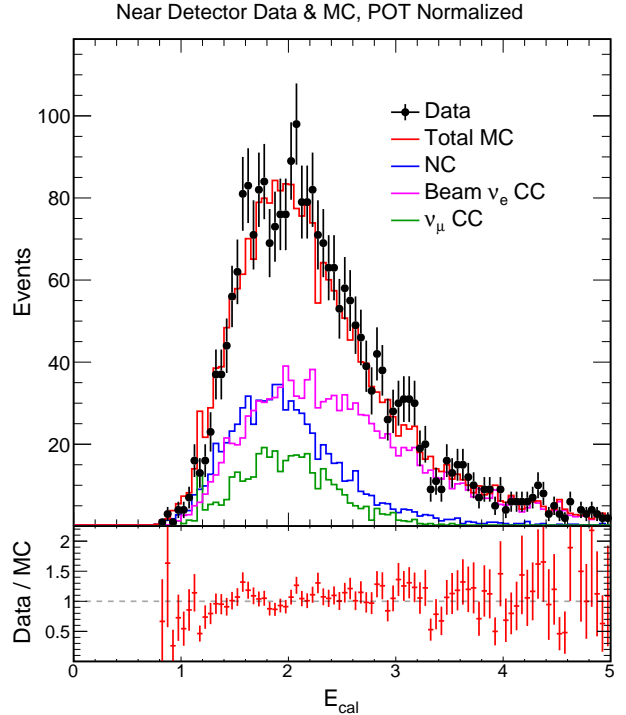

(a) Reconstructed neutrino energy

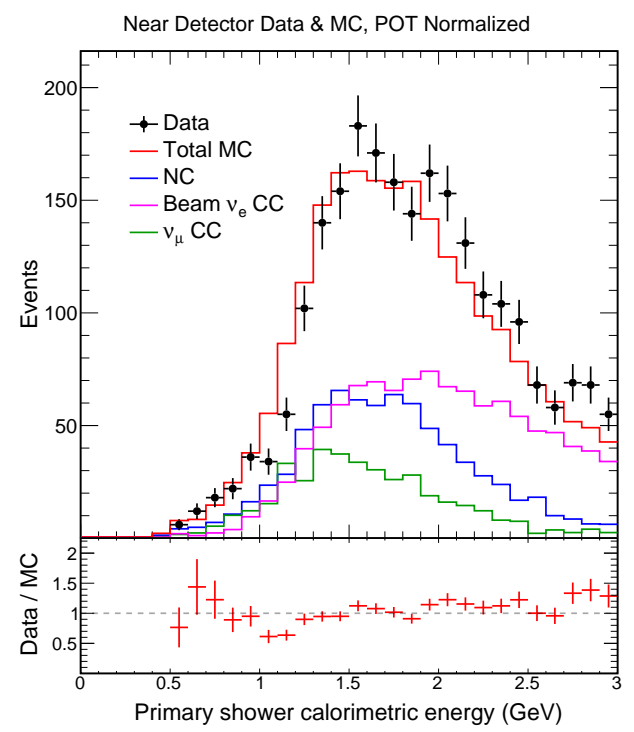

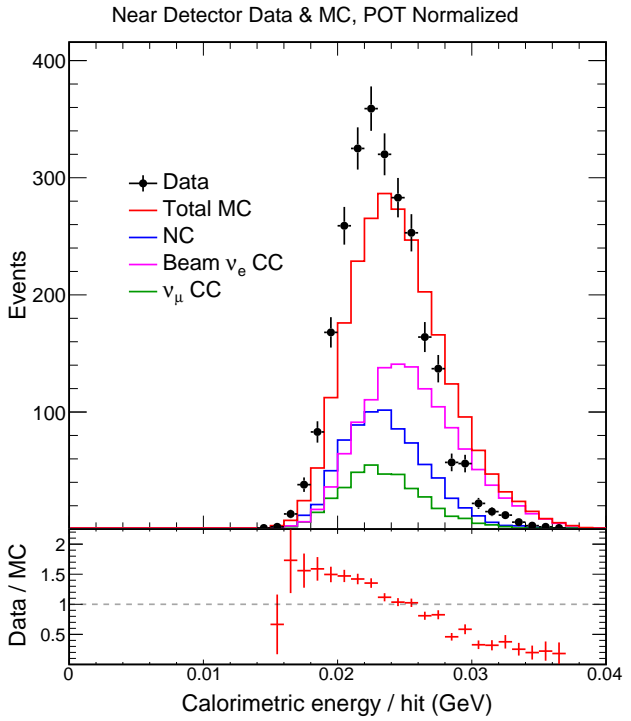

(b) Energy per hit in slice

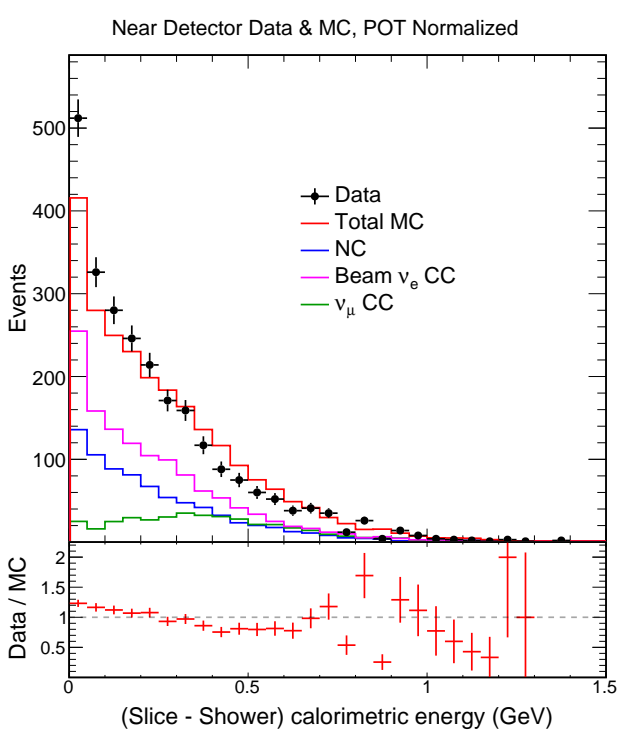

(c) Reconstructed energy of the leading (d) Energy in slice that is not on the leading shower in the event shower, or the hadronic energy in the event.

Figure 9.5: Data and MC comparison of ND LID selected events. 

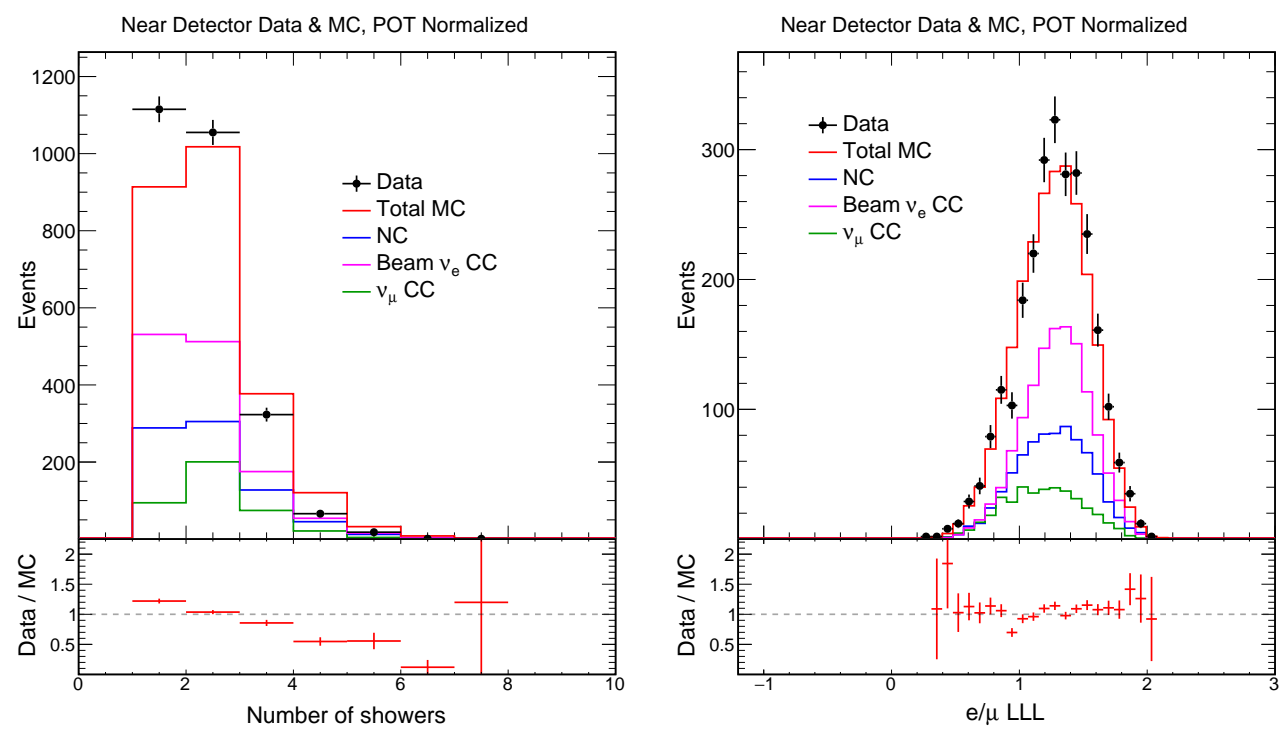

(a) Number of reconstructed 3D showers in (b) Electron-muon longitudinal logevent.

likelihood difference

Figure 9.6: Data and MC comparison of ND LID selected events. The MC is broken down by interaction type.

\subsubsection{ND $\nu_{\mu}$ Event Selection}

The prediction of the $\nu_{\mu}$ to $\nu_{e}$ oscillation signal in the far detector is based on the observed $\nu_{\mu}$ CC spectrum in the near detector. Any data-MC disagreement observed in the $\nu_{\mu}$ population is used to correct the ND and FD MC (see chapter 11).

The $\nu_{\mu} \mathrm{CC}$ selection criteria in the ND are listed in table 9.3. Unlike the $\nu_{e}$ selection, the selection of $\nu_{\mu}$ 's in the ND in an energy range relevant to oscillations requires the use of the muon catcher. The reconstructed muon track length and energy (figure 9.7a and $9.7 \mathrm{~b})$ are in reasonably good agreement. The reconstructed $\nu_{\mu} \mathrm{CC}$ energy and hadronic energy in $\nu_{\mu}$ events are shows in figures $9.7 \mathrm{c}$ and $9.7 \mathrm{~d}$ respectively and exhibit similar data-MC discrepancies to those outlined in section 9.1.1.

Figure 9.8 is the distribution of the $\nu_{\mu}$ PID, ReMID for all ND events that pass 
$\nu_{\mu}$ preselection. The low ReMID values are dominated by neutral currents and highly inelastic $\nu_{\mu} \mathrm{CC}$ interactions where the muon is not prominent. Here too, the data and simulation differ in shape.

\begin{tabular}{|l|l|}
\hline Cut & Value \\
\hline Quality & $\begin{array}{l}\text { At least one reconstructed Kalman track, } \\
\text { number of hits in slice }>20, \\
\text { number of contiguous planes with hits in slice }>4 \\
\text { Containment } \\
\text { Number of cells from edge }>1, \\
\text { first and last plane in slice }>1 \text { and }<212 \text { resp. } \\
\text { start } z \text { of the most muon-like track }<1275 \mathrm{~cm} \text { (not in muon catcher) } \\
\text { visible hadronic energy in muon catcher }<0.03 \mathrm{GeV} \\
\text { Projected distance to detector edge of the most muon-like track : } \\
\text { in the forward direction }>4 \text { cells } \\
\text { in the backward direction }>8 \text { cells } \\
\text { ReMID }>0.75\end{array}$ \\
\hline
\end{tabular}

Table 9.3: $\nu_{\mu}$ CC selection criteria in the ND 

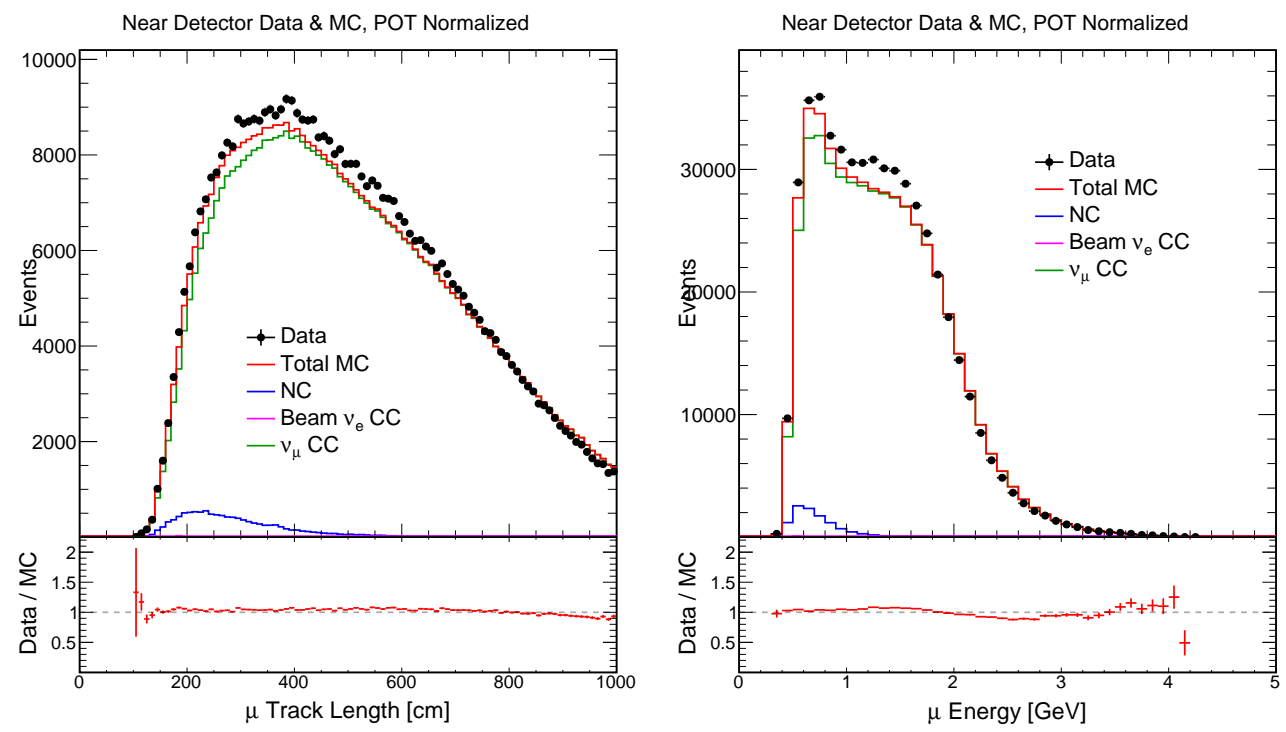

(a) Length of the candidate muon track

(b) Reconstructed energy of the candidate muon
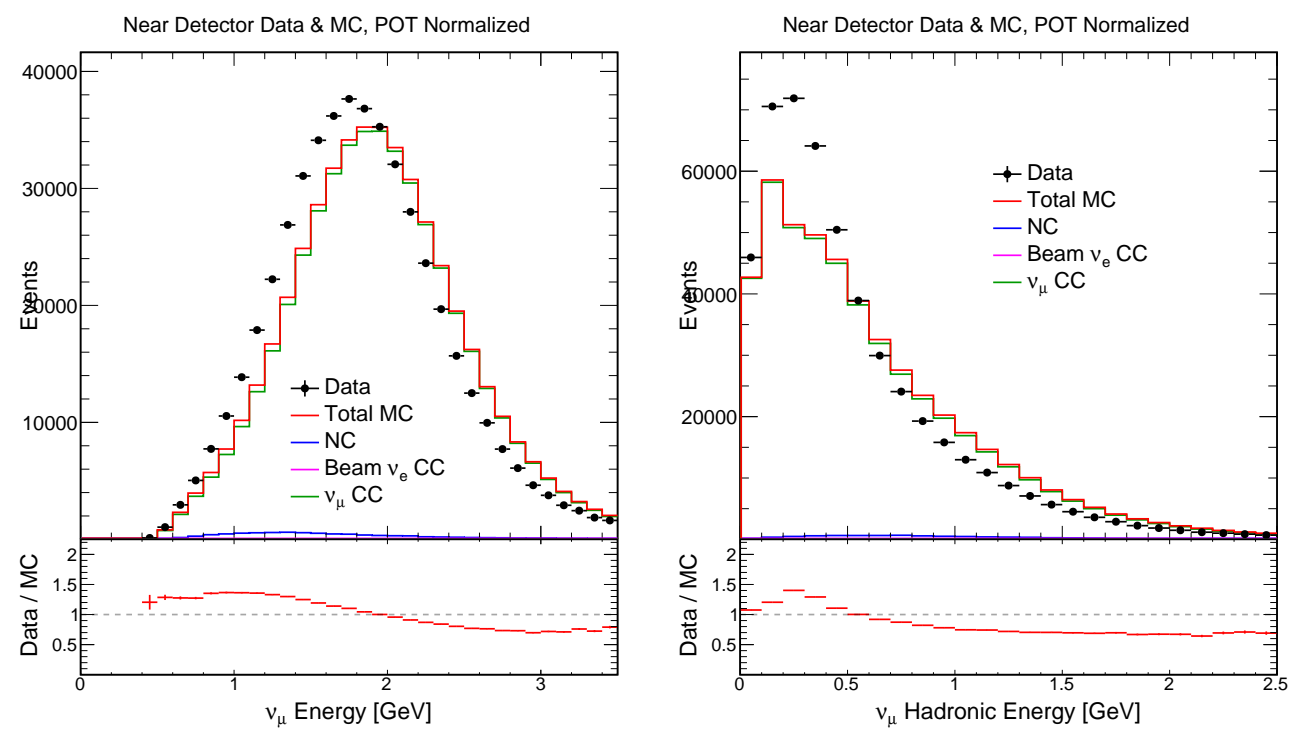

(c) Reconstructed neutrino energy of $\nu_{\mu} \mathrm{CC}$ (d) Reconstructed hadronic energy in $\nu_{\mu}$ selected events $\mathrm{CC}$ selected events

Figure 9.7: Reconstructed quantities for candidate muon tracks and $\nu_{\mu}$ CC selected events in the near detector data and MC. 


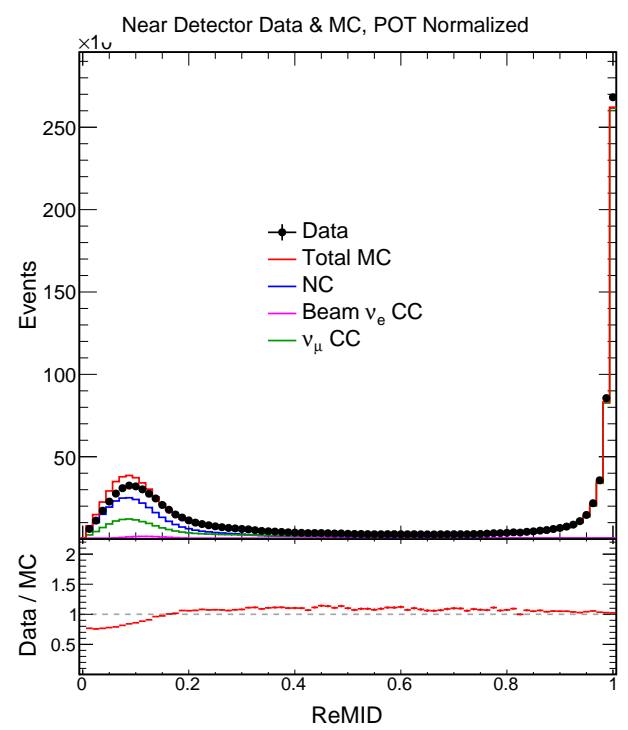

Figure 9.8: Performance of ReMID, the $\nu_{\mu}$ CC PID, on the ND data and MC.

\subsection{Interpretation}

From previous discussions, it is evident that the ND data differ from simulation in some fundamental way that is not yet completely understood. For the low statistics analysis that is presented in this thesis, such differences do not bear too heavily on the sensitivity of the final result. However, for future analyses with larger data samples, systematic errors will become important and these problems will need to be addressed. This section presents two approaches that have so far been explored as an explanation of the observed differences: adjusting the interaction type scales and adjusting the interaction mode scales in the simulation (see table 9.4). 


\begin{tabular}{|l|l|}
\hline Interaction Type & Neutral Current (NC), \\
& Beam $\nu_{e} \mathrm{CC}$ \\
& $\nu_{\mu} \mathrm{CC}$ \\
\hline Interaction Mode & $\begin{array}{l}\text { Quasi-elastic (QE), } \\
\text { Resonance (Res), } \\
\text { Deep-inelastic (DIS) } \\
\text { Coherent }\end{array}$ \\
\hline
\end{tabular}

Table 9.4: Description of interaction type and mode

\subsubsection{Fitting For Interaction Type}

The LID distribution in data disagrees in shape with the simulation. The data has fewer events than simulation in the low-LID region, and more events than simulation in the high signal region. The low PID region in LID is dominated by $\mathrm{NC}$ and $\nu_{\mu} \mathrm{CC}$ interactions while the very high PID region consists mostly of beam $\nu_{e} \mathrm{CC}$ interactions. We proceeded with the assumption that the $\mathrm{NC}, \nu_{\mu} \mathrm{CC}$ and beam $\nu_{e} \mathrm{CC}$ interactions are modeled correctly but the relative proportions (flux $\times$ cross-section) are not correct.

The events selected by cuts $0.6<\mathrm{LID}<0.8$, contain very little beam $\nu_{e} \mathrm{CC}$, so this component is held fixed. The neutrino energy distribution of these events (figure 9.10a) was used to fit for the scale of $\mathrm{NC}$ and the $\nu_{\mu} \mathrm{CC}$ components in the $\mathrm{MC}$ by minimizing the $\chi^{2}$ for a Poisson distribution:

$$
\begin{gathered}
-2 \log \mathcal{L}=-2 \sum_{i=1}^{N} o_{i} \log e_{i}-e_{i}-\log o_{i} ! \\
\Longrightarrow \chi^{2} \simeq 2 \sum_{i=1}^{N} o_{i} \log \left(\frac{o_{i}}{e_{i}}\right)+e_{i}-o_{i}
\end{gathered}
$$

where $o_{i}$ and $e_{i}$ are the observed and expected event counts in the $i$ th bin. The scale 
factors from this fit were used to scale the $\mathrm{NC}$ and $\nu_{\mu} \mathrm{CC}$ components in the energy distribution of events in LID region from 0.8 to 0.95 , to fit for the beam $\nu_{e}$ CC scale. The result of this exercise is in table 9.5. The $\chi^{2}$ distributions of the two fits are shown in figure 9.9. The most surprising aspect of this fit is that it wants to reduce the $\nu_{\mu} \mathrm{CC}$ component by $54 \%$ in the LID sideband. The reconstructed neutrino energy of events in ranges $0.6<$ LID $<0.8$ and the LID distribution in this region before and after the derived scale factors are applied are shown in figure 9.10
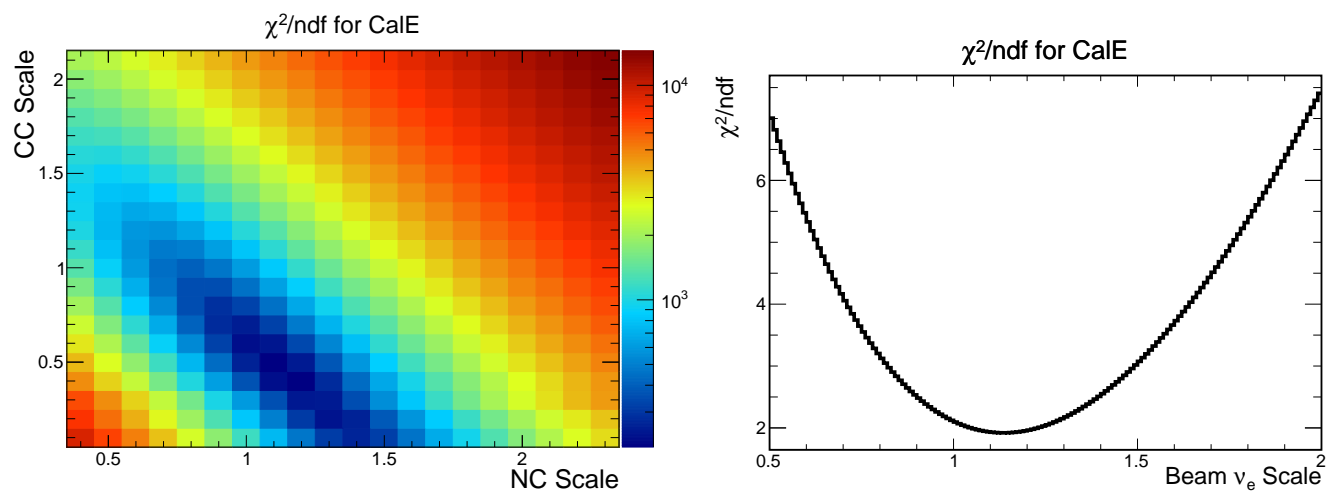

Figure 9.9: $\chi^{2}$ distributions of fit for NC, $\nu_{\mu} \mathrm{CC}$ and beam $\nu_{e} \mathrm{CC}$ scales in ND.

\begin{tabular}{ll}
\hline Interaction Type & Scale Factor \\
\hline $\mathrm{NC}$ & 1.12 \\
$\nu_{\mu} \mathrm{CC}$ & 0.46 \\
Beam $\nu_{e} \mathrm{CC}$ & 1.14 \\
\hline
\end{tabular}

Table 9.5: Result of fit for $\mathrm{NC}, \nu_{\mu} \mathrm{CC}$ and beam $\nu_{e} \mathrm{CC}$ scales using reconstructed energy distribution of events in the LID sideband from 0.6 to 0.95 . 


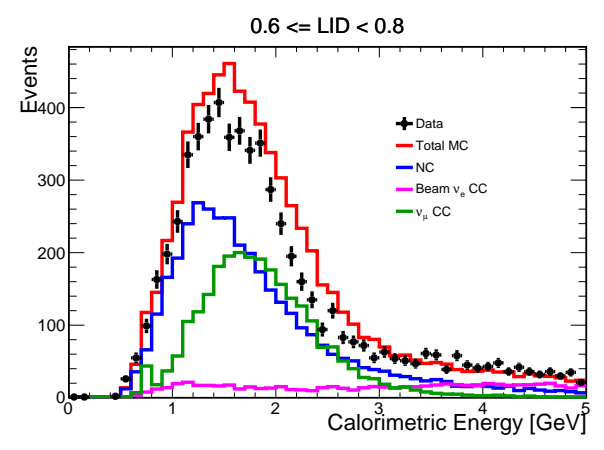

(a) Reconstructed neutrino energy of events with $0.6<=$ LID $<=0.8$, before interaction types are scaled

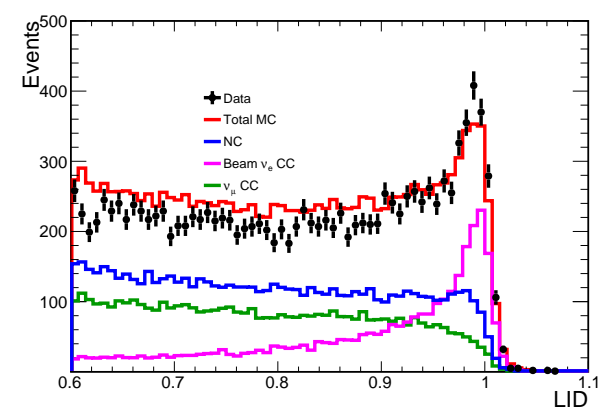

(c) LID, before interaction types are scale

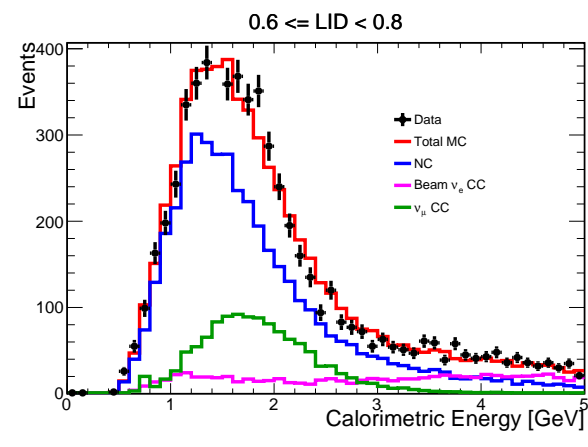

(b) Reconstructed neutrino energy of events with $0.6<=$ LID $<=0.8$, after interaction types are scaled

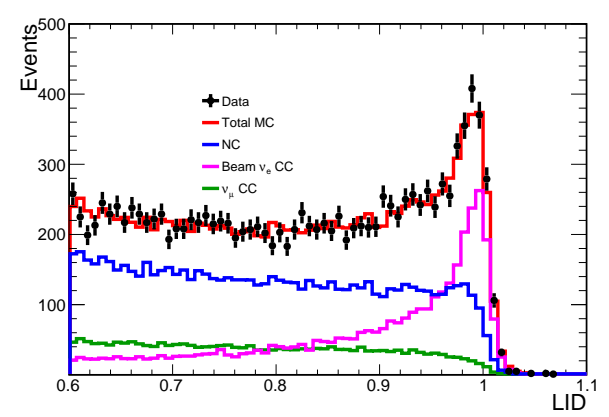

(d) LID, after interaction types are scale

Figure 9.10: Result of scaling interaction types in MC by the factors in table 9.5.

\subsubsection{Fitting For Interaction Mode}

Neutrino interactions with nucleons are broadly divided into elastic and inelastic. The recoiling nucleus remains intact in case of elastic interactions, which dominate at low momentum transfer $\left(Q^{2}\right)$. In case of charged-current, an interaction can not be elastic because some energy is converted to the mass of the final-state lepton and there is an exchange of charge. These interactions are called quasi-elastic (QE), and are characterized by a track or a shower due to the primary lepton, and a small amount of 
hadronic activity. At high momentum transfer, the neutrino interacts with the constituent quarks of a nucleon, which breaks apart as a consequence of the interaction. These are deep-inelastic (DIS) interactions and are usually characterized by significant hadronic activity. Inelastic interactions at low $Q^{2}$ usually proceed via excitation of nucleons into resonances such as $\Delta$. If the nucleus as a whole recoils due to interaction with a neutrino, it is known as coherent scattering.

Interaction modes are a convenient conceptual tool in understanding interactions of neutrinos with nucleons, but there are no distinct boundaries that separate one process from another in practice. The neutrino-nucleon interactions occur with a continuum of inelasticity as the momentum transfer rises. Final state hadrons can also interact in the nuclear medium as they exit the nucleus, so that the apparent final state appears very different from the one produced. All of this put together makes for a very challenging problem in modeling and measuring interaction mode cross-sections.

The near detector data and simulation strongly differ in the behavior of the hadronic component of the interactions. As a measure of hadronic energy, we subtract the energy of the electron shower candidate from the event energy. The data prefer, on average, lower hadronic energy and multiplicity. The hadronic energy distribution differs significantly for different interaction modes. From figure 9.11a, it appears that the data favor fewer DIS and more QE interactions. 

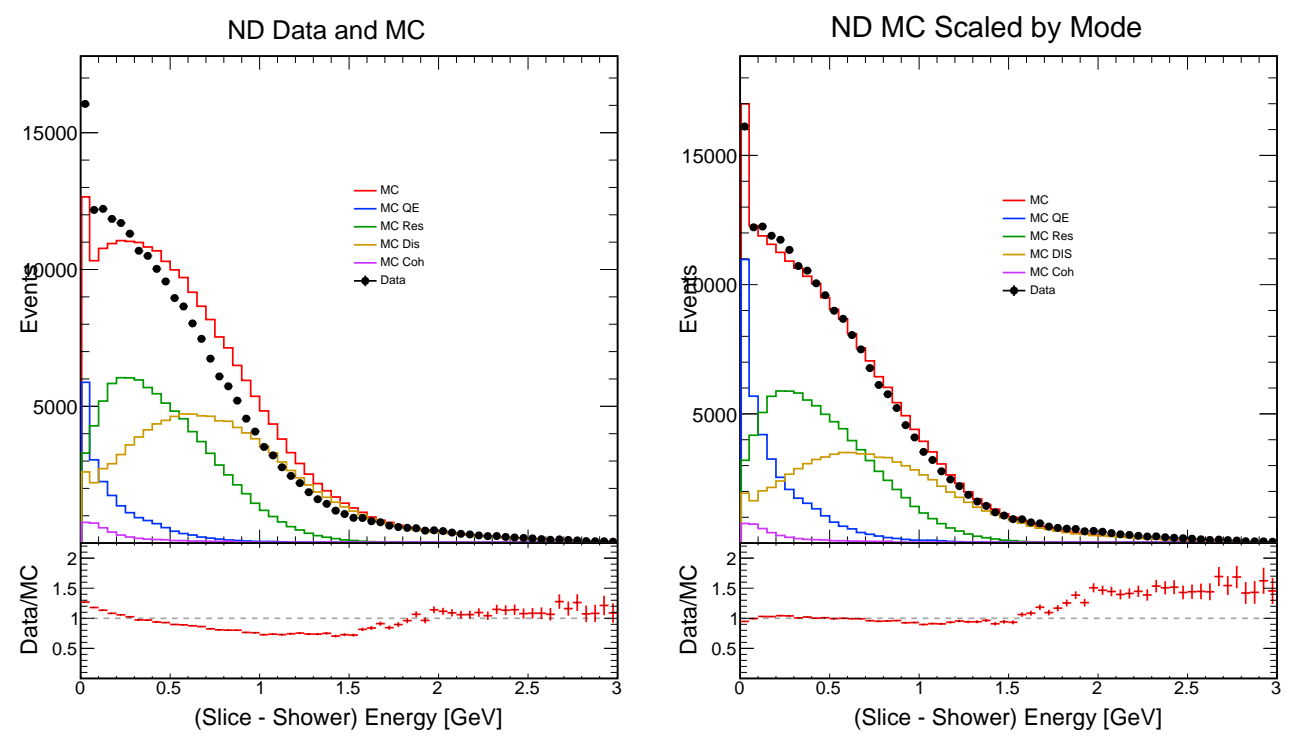

(a) Hadronic energy in preselected ND (b) Hadronic energy in preselected ND events, MC split by mode events, after fitting, MC split by interaction type

Figure 9.11: Hadronic energy in ND preselected events, split by interaction mode, before and after the fit to interaction mode scales.

A fit to the hadronic energy distribution of $\nu_{e}$ preselected events was performed to obtain QE, Res and DIS scale factors that best represent the data. Since coherent interactions are such a small fraction of the total, their scale was kept fixed. The result of this fit are shown in table 9.6. 


\begin{tabular}{ll}
\hline Interaction Mode & Scale Factor \\
\hline Quasi-elastic & 1.86 \\
Resonance & 0.97 \\
Deep-inelastic & 0.74 \\
Coherent & 1.00 (fixed) \\
\hline
\end{tabular}

Table 9.6: Result of fit for QE, resonance and DIS scales using reconstructed hadronic energy distribution of $\nu_{e}$ preselected events in the ND.

The fit favors a near doubling of the QE interactions in the MC. The fitted scale factors perform remarkably well in bringing the simulation closer to the data across a variety of very different variables (see figures 9.13, 9.14 and 9.15), which gives some confidence that this is a step in the right direction. Neutrino cross-sections are not very well-measured in general. The energy range of interest to $\mathrm{NO} \nu \mathrm{A}$, around $2 \mathrm{GeV}$ neutrino energy, is still less understood due to the falling QE and rising resonance and DIS cross-sections (see figure 9.12). Nevertheless, such a large discrepancy in the simulation is surprising. It is unlikely that the quasi-elastic cross-section is nearly a factor of two lower in the simulation and the high scale factor more likely indicates that the number of interactions from DIS with low hadronic activity is underrepresented. However, we are not concerned here with determining the correct relative cross-sections of various modes, but we are concerned with finding a model that adequately describes our data. This appears to be achieved to some degree with the fit discussed here. More careful studies of interaction modes and comparisons with other neutrino experiments are necessary to further shed light on this topic. 


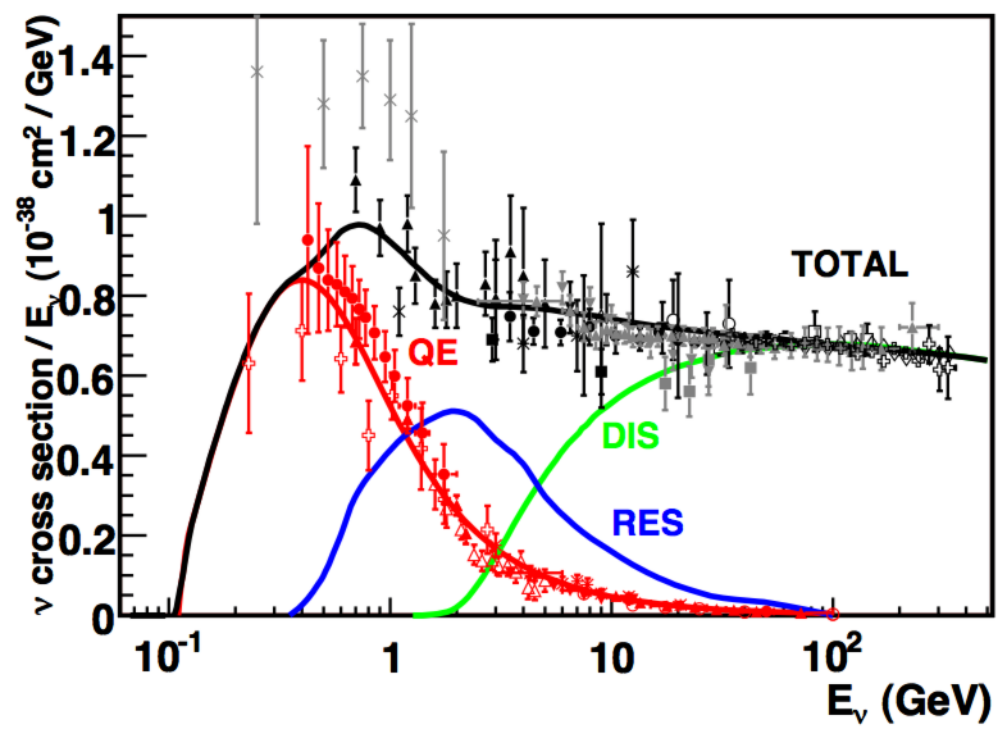

Figure 9.12: Cross-section as a function of neutrino energy in different modes. For further description of the plot, see [4] 

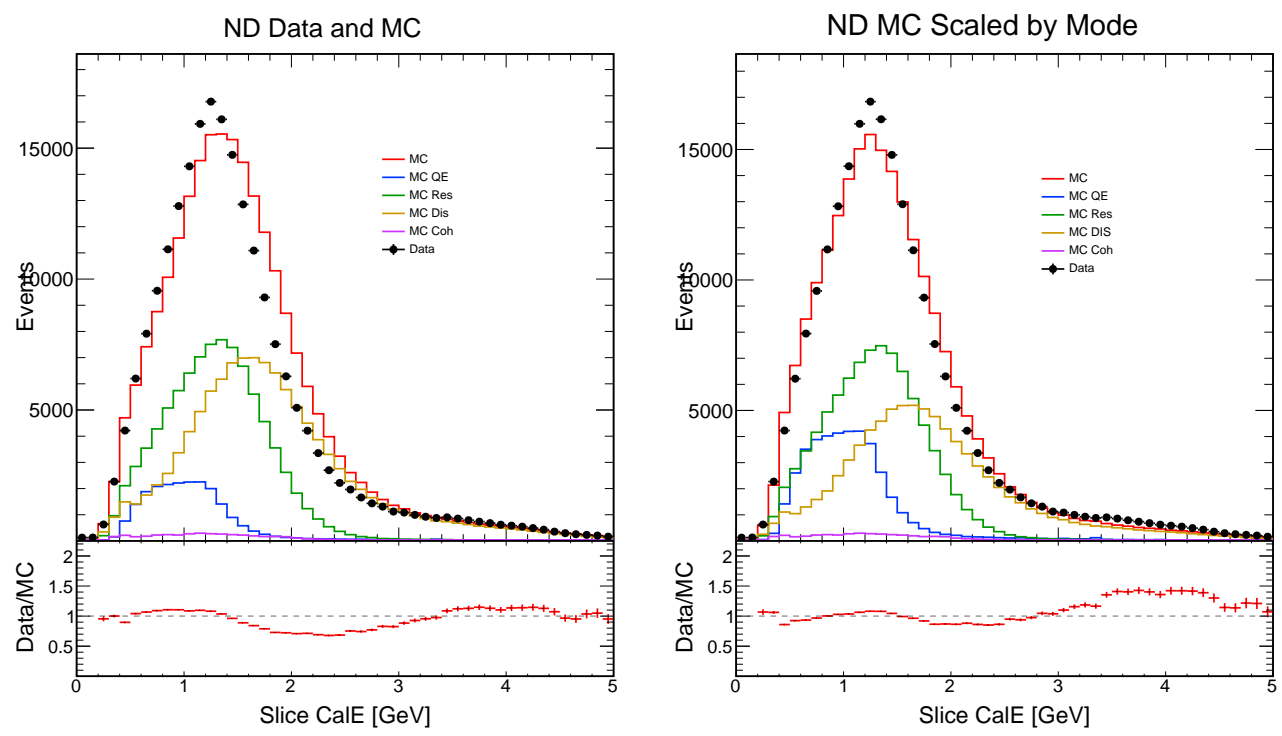

(a) Reconstructed neutrino energy, before (b) Reconstructed neutrino energy, after scaling by mode scaling by mode
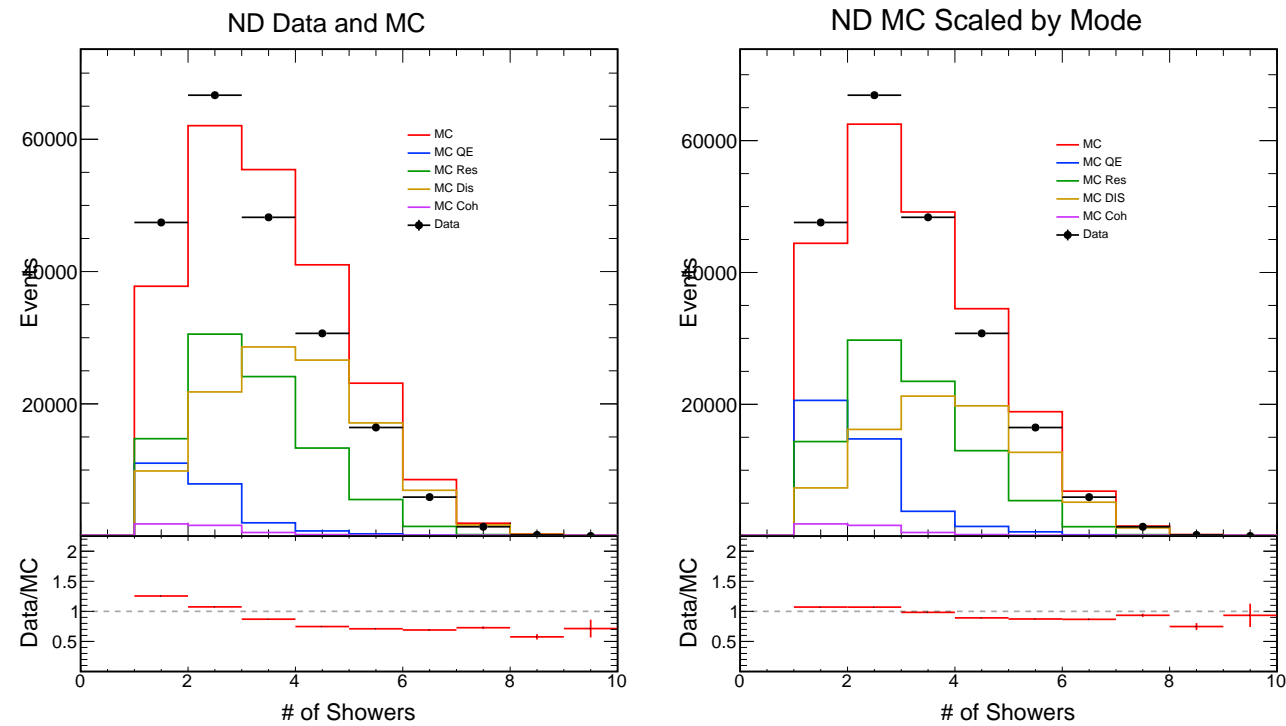

(c) Number of reconstructed showers per (d) Number of reconstructed showers per event, before scaling by mode event, after scaling by mode

Figure 9.13: Data and MC comparison of ND $\nu_{e}$ preselected events. The distributions on the left are base simulation, split by interaction mode and those on the right have the scale factors in table 9.6 applied, and are split by interaction type. 

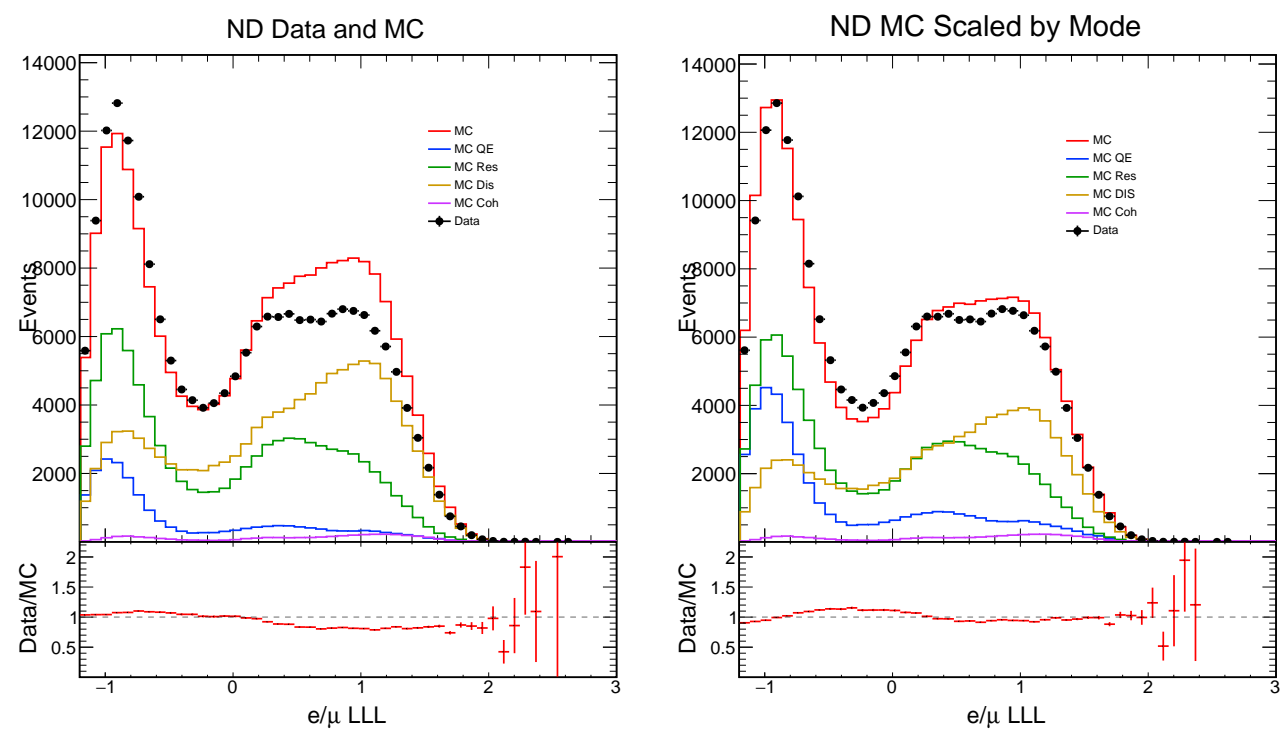

(a) Log-likelihood difference between elec- (b) Log-likelihood difference between electron and muon hypothesis, before scaling tron and muon hypothesis, after scaling by by mode mode
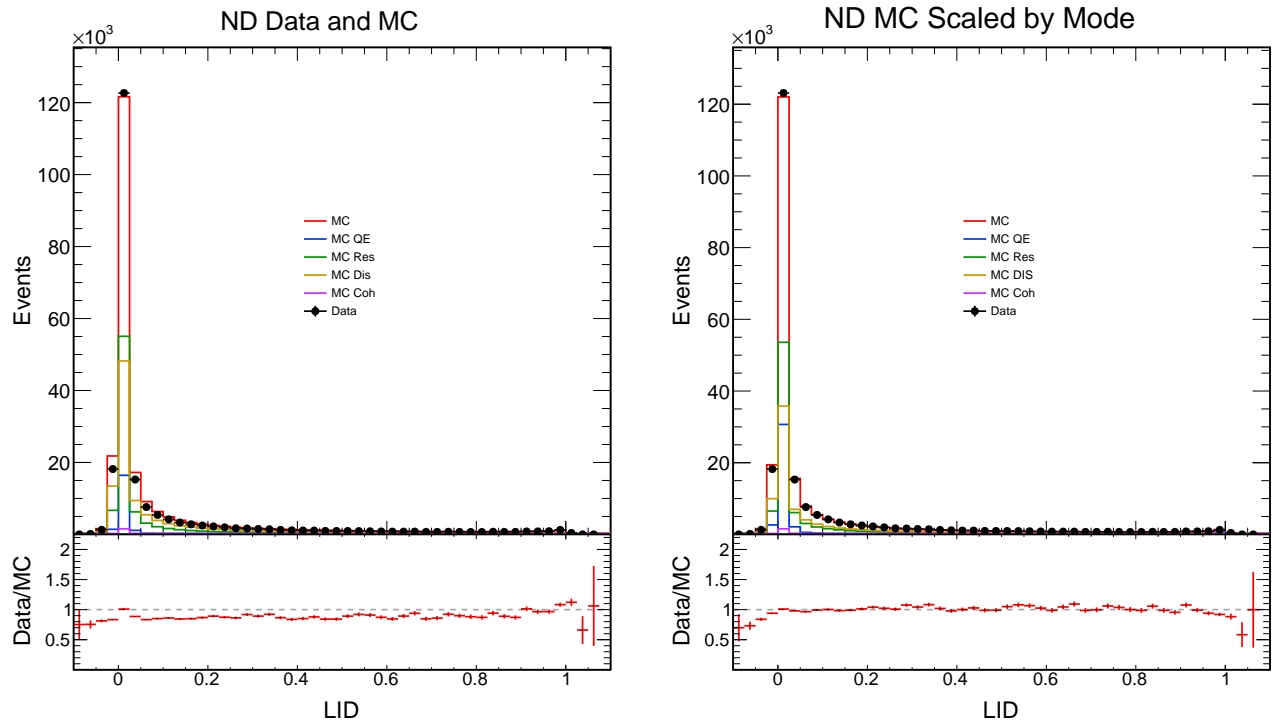

(c) LID, before scaling by mode

(d) LID, after scaling by mode

Figure 9.14: Data and MC comparison of ND $\nu_{e}$ preselected events. The distributions on the left are base simulation, split by interaction mode and those on the right have the scale factors in table 9.6 applied, and are split by interaction type. 

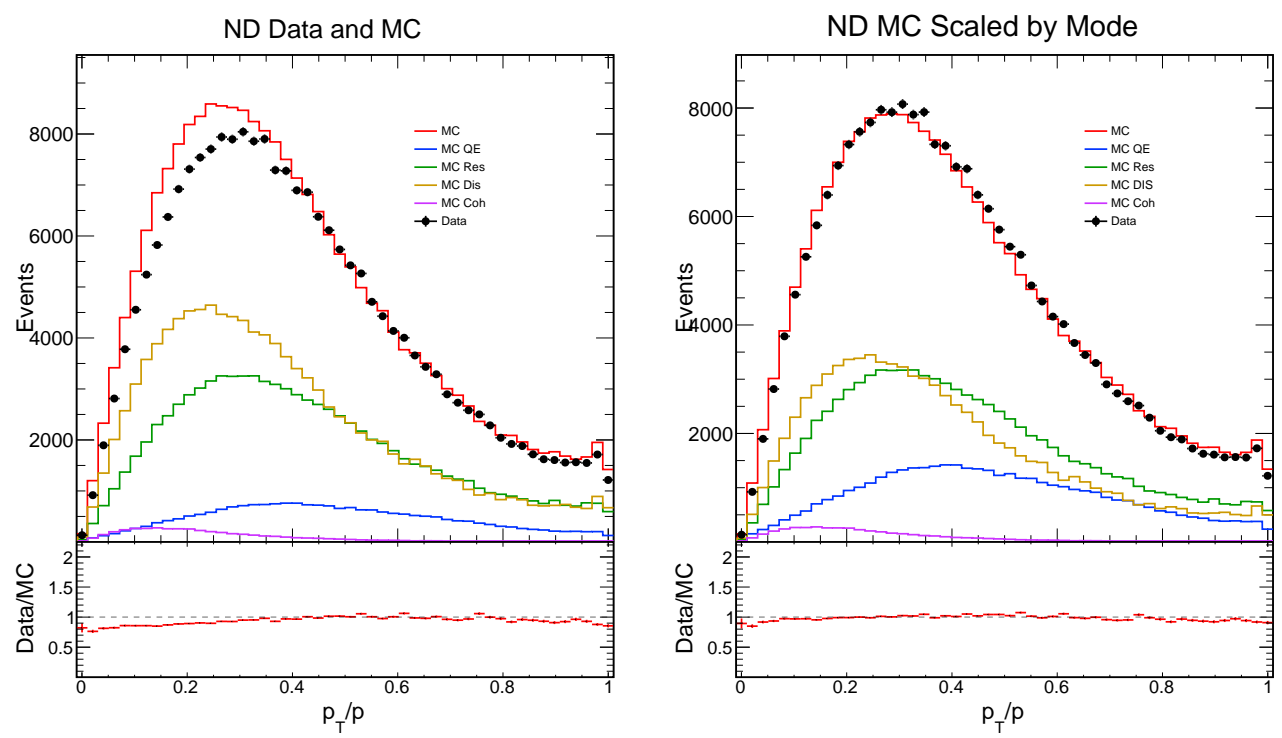

(a) Transverse momentum fraction, before (b) Transverse momentum fraction, after scaling by mode

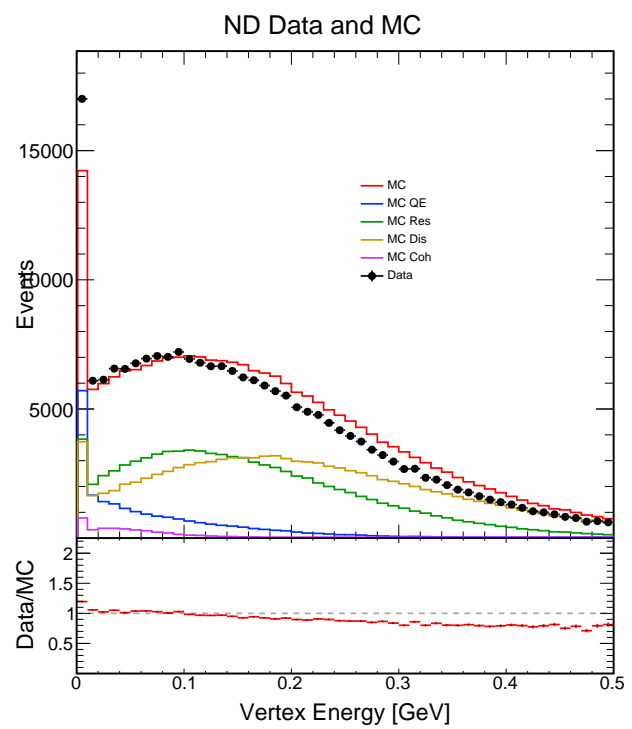
scaling by mode

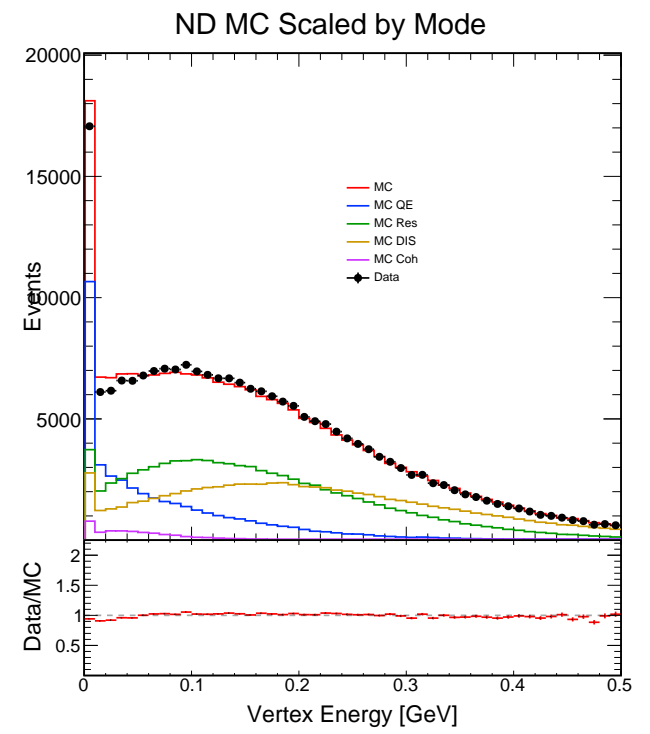

(c) Energy within 8 planes of the vertex, (d) Energy within 8 planes of the vertex, before scaling by mode after scaling by mode

Figure 9.15: Data and MC comparison of ND $\nu_{e}$ preselected events. The distributions on the left are base simulation, split by interaction mode and those on the right have the scale factors in table 9.6 applied, and are split by interaction type. 


\section{Summary}

The near detector data are a large statistic sample of neutrino interactions that can be scrutinized to test assumptions in our simulation of neutrino interactions. When fairly loose selection ( $\nu_{e}$ preselection cuts) is applied to the ND data and MC, there are significant differences. There are indications that the main source of deviations is the simulation of hadronic showers. These data-Monte Carlo anomalies are similarly observed in the $\nu_{\mu}$ selected sample too. Once $\nu_{e}$ PID cut, that nearly guarantees the presence of electromagnetic activity in the event, is applied, the data-MC differences are smaller.

To bring the simulation in better agreement with data, two independent approaches have been attempted. One is to fit for the interaction type scales in the MC (NC, $\nu_{\mu} \mathrm{CC}$ and beam $\nu_{e} \mathrm{CC}$ ), the other is to fit for the scales of interaction modes (QE, resonance, DIS). While both approaches show promising results, the latter appears to get to the root of the problem better than the former, and will be used later in this thesis. The results of both approaches are somewhat surprising. A systematic review of the simulation configuration, comparisons with data from other neutrino experiments, and further analysis of the $\mathrm{NO} \nu \mathrm{A}$ near detector data in the coming months should help clarify the matter further. 


\section{Chapter 10}

\section{Muon Removed Electron Events}

The near detector data allows a close look at the neutrino backgrounds to the $\nu_{e}$ appearance signal, but offers little information about what may be expected of the signal events. Muon removed electron, or MRE, events are a construct that remedy this. These events are constructed from obvious $\nu_{\mu} \mathrm{CC}$ interactions by removing the primary muon track and simulating an electron in its place, as shown in figure 10.1. This procedure can be performed on both, data and simulation. This combines electron showers, that we simulate well, with hadronic shower from data, which are not well simulated, and helps understand how the mismodeling of hadronic showers impacts $\nu_{e}$ selection.

In this chapter, the construction of MRE events is described in some detail, followed by data and $\mathrm{MC}$ comparisons of MRE samples and its implications for the $\nu_{e}$ appearance signal.

\subsection{MRE Event Generation}

The MRE events are built from $\nu_{\mu} \mathrm{CC}$ events where the muon is removed from the event. This intermediate stage is called Muon Removed Charged Current, or MRCC. 


\subsubsection{Muon Removal}

The removal of muon information is done at the most basic level of data, the cell hits. The hits in each channel that belong to the muon are removed from the event. In case the muon and a hadron resulting from the interaction are in the same horizontal or vertical plane, it is possible that part of the energy deposited in a cell on the muon track is from a non-muon source. In such a cell, the hit must not be completely removed. Instead, the ADC value for a hit on the muon track is scaled down as follows:

$$
\text { new } A D C=\text { original } A D C \times(1-\text { muonWeight })
$$

Here muonWeight is the fraction of the total energy deposited in the cell by the

muon. If all the energy in a cell is due to the muon, then muonWeight must be 1 and new $A D C=0$. If the new $A D C$ is 0 or below threshold, the hit is removed from the list of $m r c c$ hits. 


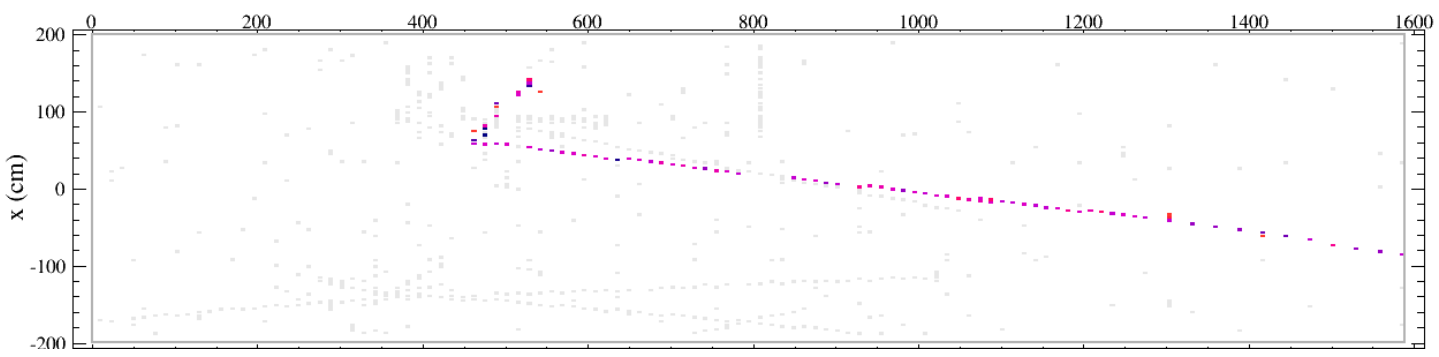

(a) A candidate $\nu_{\mu} \mathrm{CC}$ interaction in ND data

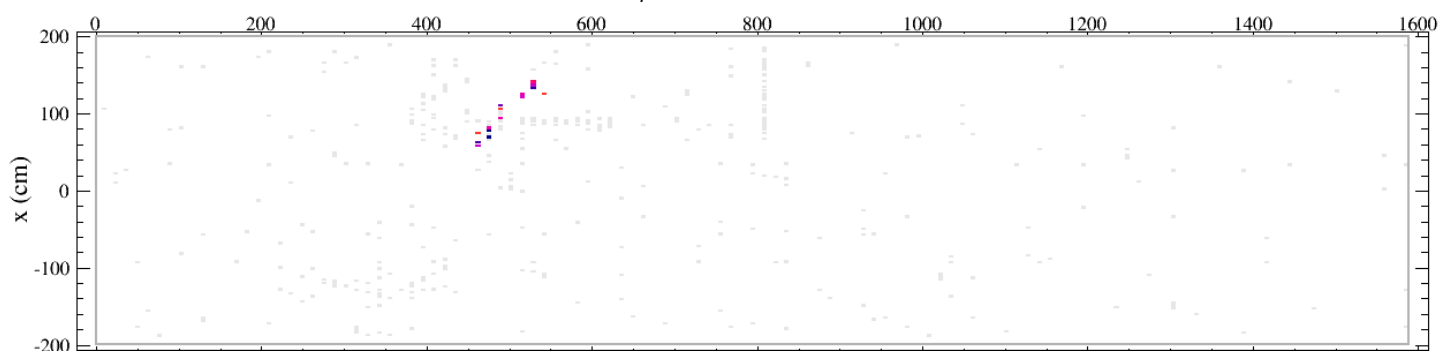

(b) The muon removed or MRCC version of the event

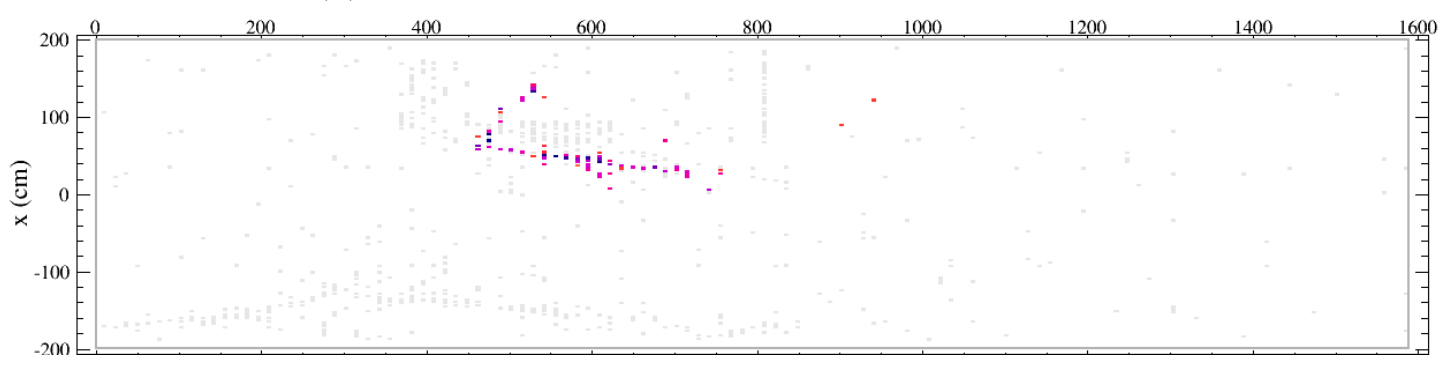

(c) A simulated electron is inserted in place of the muon to make an MRE event.

Figure 10.1: MRE event creation process

\subsubsection{Muon and Hadron Energy Disentaglement}

Muons lose energy in a medium mostly via ionization of atoms along their trajectory. The mean energy deposited by a muon per unit length is a characteristic of the medium and the kinematics. The $d E / d x$ of muons at energies typical in $\mathrm{NO} \nu \mathrm{A}$ is close to the minimum of the Bethe-Bloch curve which is used as an energy unit equivalent. This unit is termed Minimum Ionizing Particle energy equivalent or MIP. The muon removal algorithm takes advantage of this property of muons to dis-entangle the energy deposited 
by the muon in a cell from that deposited by a hadron in the same cell.

The region at the start of the muon candidate track is often characterized by high activity as the scattering nucleon breaks apart and it becomes likely that the muon and the hadrons deposit energy in the same cells. This region where the hadronic contamination of the muon track is very likely is called the vertex-region. If one or more particles other than the muon deposit energy in a cell on the muon track, the $d E / d x$ in that plane would be more than that expected from the muon alone. The plot in figure 10.2a shows the $d E / d x$ distribution of planes with energy due to the muon only and those with energy from non-muon sources.
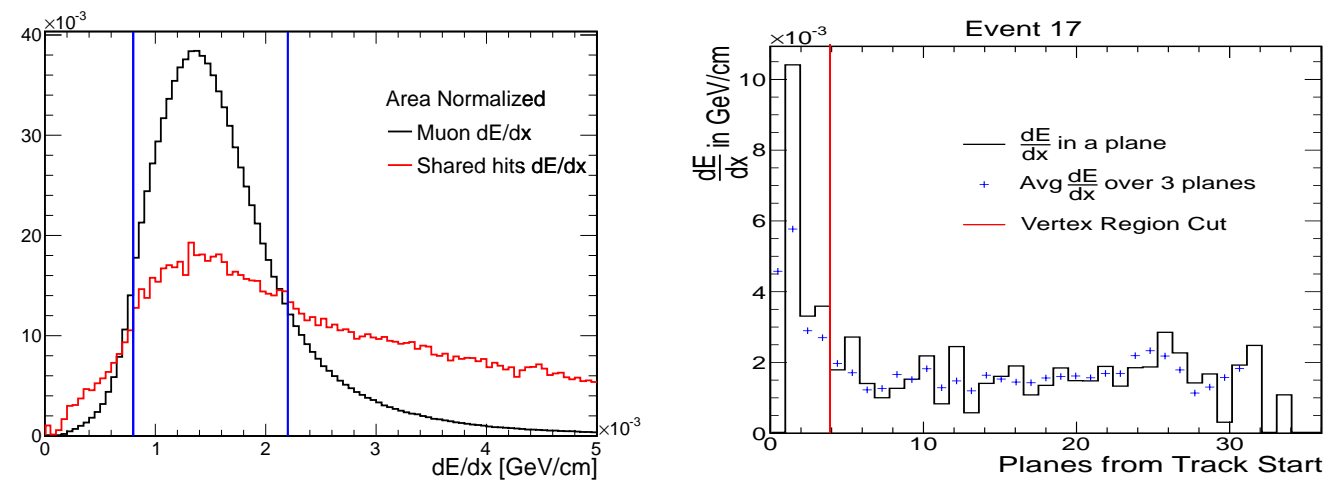

(a) $d E / d x$ per plane on the muon track. The (b) $d E / d x$ profile of a muon in a $\nu_{\mu} \mathrm{CC}$ inblue lines mark the muon $d E / d x$ cut range. teraction. The red line marks the end of the vertex region

Figure 10.2: $d E / d x$ of muons with and without hadronic contamination.

\section{Determination of Vertex-Region}

The removal algorithm determines the vertex-region dynamically for every event. Since the muon track may be highly contaminated in one view while it is very pure in the other view, the determination of vertex-region is done independently for each detector view. From the start of the muon track, the $d E / d x$ values for each set of three consecutive 
planes in the same view $(i, i+1$ and $i+2)$ are averaged. If the averaged $d E / d x$ values drop to values consistent with a MIP, and stay low for the next three averaged values, the vertex-region is considered to be over. $10.2 \mathrm{~b}$ shows the $d E / d x$ profile for a muon track in a $\nu_{\mu} \mathrm{CC}$ interaction. The red line marks the end of the vertex region as found by this procedure. The blue lines in figure 10.2a mark the $d E / d x$ range that is considered to be compatible with the muon.

\section{Determination of muonWeight}

The muonWeight for all hits on the muon track outside the vertex region is set to 1 . Inside the vertex region, if the $d E / d x$ value in a given plane is within the range 0.0008 $0.0022 \mathrm{GeV} / \mathrm{cm}$, the plane is consistent with MIP assumption for the muon and the muonWeight for the hits in the plane is set to 1 .

If the $d E / d x$ in a plane is greater than $0.0022 \mathrm{GeV} / \mathrm{cm}$, the following steps are taken:

1. The hits in that plane on the track are ordered by their closeness to the track in that plane.

2. These hits are looped over, starting with the one closest to the trajectory point. The energy of the cells is summed and the $d E / d x$ recalculated for every cell-energy added and checked for consistency with the MIP range.

3. If consistency with MIP range is attained, the rest of the hits in the plane get a muon weight of 0 .

4. If the $d E / d x$ is greater than the MIP range upper-bound, the hit then gets a muonWeight that would be just enough to make the $d E / d x$ equal the MIP peak. The rest of the hits in the plane get a muonWeight of 0 .

5. If the $d E / d x$ is less than the MIP range lower-bound, the next closest hit energy is added. 
If the $d E / d x$ in a plane is less than $0.0008 \mathrm{GeV} / \mathrm{cm}$, the following steps are taken:

1. The hits in that plane in the same slice but not on the muon track, and within 5 cells from the muon track, are ordered by their closeness to the track in that plane.

2. Starting from the closest such hit, its energy is added to the plane energy.

3. If consistency with MIP range is attained, the muonWeight of that hit is set to 1 .

4. If the $d E / d x$ is less than the MIP range lower-bound, the next closest hit energy is added.

5. If the $d E / d x$ is greater than the MIP range upper bound, the hit then gets a muonWeight that would be just enough to make the $d E / d x$ equal the MIP peak.

Cumulatively, this procedure correctly tags $\sim 76 \%$ of hits with hadronic energy, and wrongly tags $\sim 24 \%$ of pure muon hits as having hadronic contribution.

\subsubsection{Performance of Muon-Removal}

If muon removal works perfectly, it should remove only and all the energy left by the muon in the detector, while leaving the hadronic energy in the event untouched. The metrics that can assess the performance of muon removal are : fraction of muon energy left behind after removal, muFrac and fraction of hadronic energy removed in the process of muon removal, hadFrac. Both of these quantities should be zero in case of perfect muon-removal and are defined as follows:

$$
\begin{aligned}
\text { muFrac } & =\frac{\sum_{\mu \text { Hits }}\left(E_{\mu}-E_{\text {tot }} \times \frac{\text { ADC-Removed }}{\text { ADC-Original }}\right)}{\text { Total } E_{\mu}} \\
\text { hadFrac } & =\frac{\sum_{\text {had Hits }}\left(E_{\text {had }}-E_{\text {tot }} \times \frac{\text { ADC-MRCC }}{\text { ADC-Original }}\right)}{\text { Total } E_{\text {had }}}
\end{aligned}
$$

Here, $E_{\text {tot }}$ refers to the sum of all the true energy deposited in a cell, $E_{\mu}$ and $E_{\text {had }}$ are all the true energy deposited in a cell by only the muon and only the hadrons respectively. 
ADC-Original is the ADC value of the hit before removal, while ADC-MRCC is the $\mathrm{ADC}$ value left behind after removal; ADC-Removed is the difference of the two and is a measure of the energy attributed to the muon in the cell.

The performance of muon- removal in terms of these metrics is illustrated by figure 10.3. The distributions are strongly peaked at 0 for the metrics, with small scatter, indicating that muon-removal is performing well. 2-D plots of muFrac and hadFrac with respect to true muon and hadronic energies respectively indicate that most of the long tail about 0 in the fractions arises at very low energies where Poisson fluctuations can be significant.

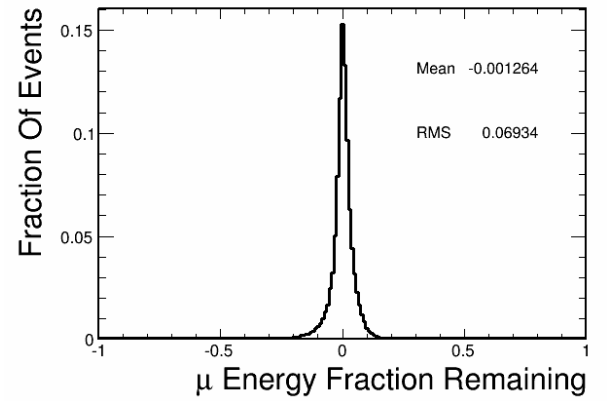

(a) Fraction of muon left behind after removal, muFrac

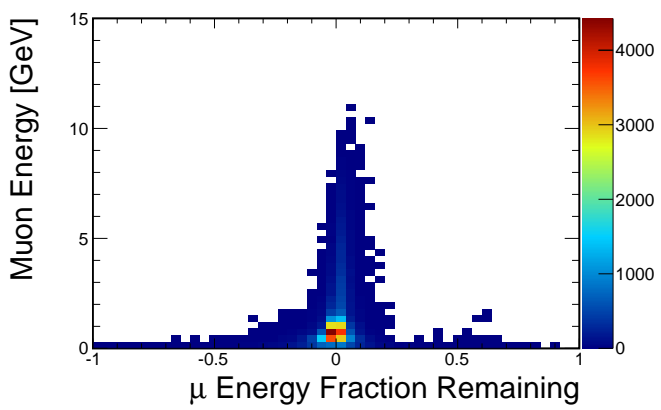

(c) muFrac vs muon energy

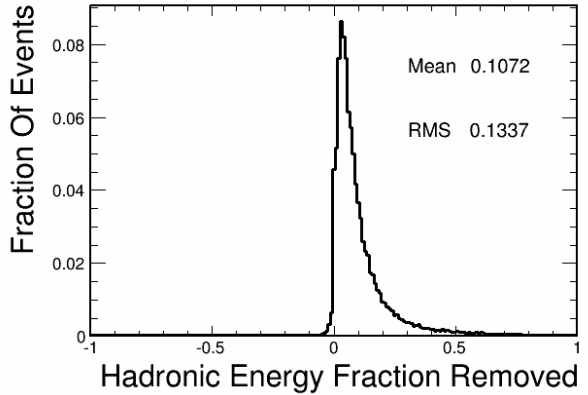

(b) Fraction of hadrons removed during muon removal, hadFrac

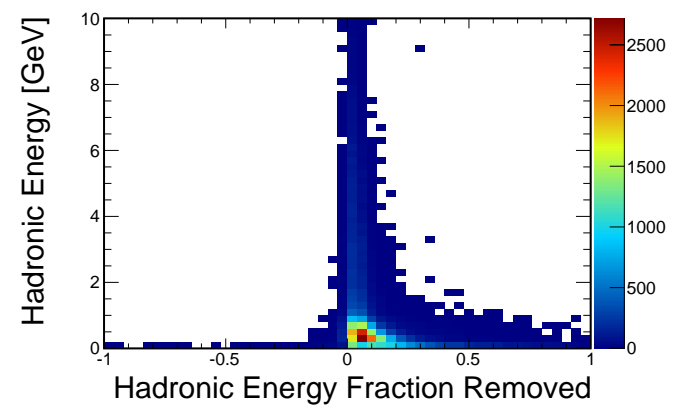

(d) hadFrac vs hadronic energy

Figure 10.3: Variables to measure the performance of muon removal 


\subsubsection{Insertion of Simulated Electron}

The most muon-like track is removed from every slice and for each muon removed from the spill, an electron is generated in its place. The information about the muon that is passed on to GEANT is the start point of the muon track, the direction of the muon ie

$\left(\hat{p}_{x}, \hat{p}_{y}, \hat{p}_{z}\right)$ and the energy reconstructed from range of the muon. Thus, the resulting electron has the same energy as the removed muon, but a different momentum. It also begins in the same place and travels in the same direction as the muon.

Once the simulated electron hits are generated, they are overlaid with the hits of the MRCC event to get the final MRE event. In case the electron produced a hit in a cell where an MRCC hit already exists, the pedestal subtracted ADC of the electron and the MRCC hit are added together to make a single hit.

\subsection{MRE ND Data-MC Comparison}

\subsubsection{MRE Event Selection}

When an MRE event is created, no cut is applied on the original interaction. The most muon like track, ie the track with the highest ReMID (see section 7.5) value is simply removed from the event. The cuts on the original event, to ensure that it is a $\nu_{\mu} \mathrm{CC}$ interaction, are applied at the time of analysis. This allows the flexibility of changing the $\nu_{\mu}$ selection cuts for optimization and systematic studies after the files are generated.

Since each spill in the ND consists of multiple neutrino interactions, and therefore, multiple slices, an MRE slice must first be matched to its parent slice. The original slice with which an MRE slice has maximum overlap in terms of cellhits, ie for which the fraction of hits in MRE slice that are in common with the original parent slice is the highest, is called the MRE parent slice. In addition to applying $\nu_{\mu}$ selection cuts to the parent slice, we need to safeguard against the loss of too many hadronic hits when the MRE interaction is being sliced. Even if the hadronic hits are not assigned to the 
same slice as the electron, where they belong, they exist in the list of all hits in a given spill. We define MRE slicing efficiency as the number of overlapping hits between the parent and MRE slices divided by number of overlapping hits between the parent slice and all the MRE hits in a spill. This slicing efficiency is required to be more than $80 \%$.

An MRE interaction whose parent passes $\nu_{\mu}$ selection criteria, and the MRE slice efficiency cut, is then subject to all the $\nu_{e}$ selection criteria developed for the ND. The cut flow efficiencies of MRE events in ND MC and data are shown in table 10.1. The efficiency of the $\nu_{e}$ selection cuts is measured with respect to events that pass all parent matching and selection criteria.

\begin{tabular}{|c|c|c|c|c|c|}
\hline & Total MC & Efficiency & Data & \multicolumn{2}{|c|}{ Efficiency } \\
\hline No Cut & 31415264 & $100.00 \%$ & 28012242 & $100.00 \%$ & \\
\hline$\nu_{\mu}$ Quality & 21570177 & $68.66 \%$ & 20914155 & $74.66 \%$ & \\
\hline$\nu_{\mu}$ Containment & 1042575 & $3.32 \%$ & 1041643 & $3.72 \%$ & \\
\hline$\nu_{\mu} \mathrm{PID}$ & 489857 & $1.56 \%$ & 513569 & $1.83 \%$ & \\
\hline MRE slicing efficiency & 459449 & $1.46 \%$ & 409132 & $1.46 \%$ & \\
\hline All parent cuts & 459449 & $100.00 \%$ & 409132 & $100.00 \%$ & \\
\hline Data quality & 457467 & $99.57 \%$ & 406306 & $99.31 \%$ & \\
\hline Reconstruction & 441542 & $96.10 \%$ & 393939 & $96.29 \%$ & \\
\hline Fiducial & 182518 & $39.73 \%$ & 158696 & $38.79 \%$ & \\
\hline Containment & 155612 & $33.87 \%$ & 138317 & $33.81 \%$ & \\
\hline Front planes & 154458 & $33.62 \%$ & 137499 & $33.61 \%$ & \\
\hline Slice hits and $E_{\text {cal }}$ & 153531 & $33.42 \%$ & 135965 & $33.23 \%$ & \\
\hline Shower length & 152217 & $33.13 \%$ & 134176 & $32.80 \%$ & \\
\hline Gap & 150988 & $100.00 \%$ & 133175 & $32.55 \%$ & $100.00 \%$ \\
\hline LID & 44254 & $9.63 \% \quad 29.31 \%$ & 47289 & $11.56 \%$ & $35.51 \%$ \\
\hline
\end{tabular}

Table 10.1: Cut flow of ND MRE data and MC 
After the $\nu_{\mu}$ selection criteria are applied, the MC has $4.7 \%$ fewer events than data. The MRE slicing efficiency is very different in data and MC and applying a cut on this quantity causes there to be more events in $\mathrm{MC}$ than data by $12.3 \%$. The efficiencies of $\nu_{e}$ preselection cuts on MRE data and MC are consistent with each other to sub-percent level. Since these events imitate $\nu_{e} \mathrm{CC}$ interactions, a large fraction of them pass the $\nu_{e}$ selection cuts.
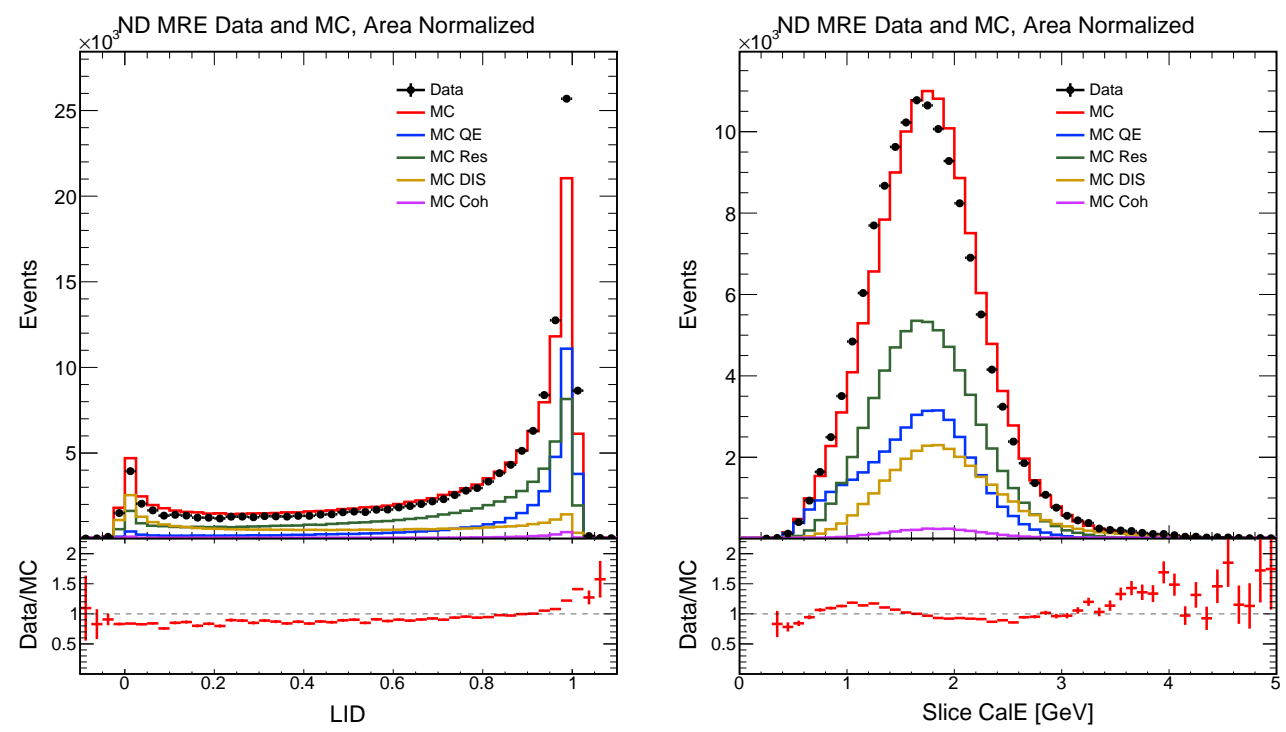

(a) LID distribution of MRE data and MC (b) Reconstructed neutrino energy of MRE data and $\mathrm{MC}$

Figure 10.4: Comparison of MRE $\nu_{e}$ preselected events in ND data and MC. MC is area normalized to the data to emphasize the shape difference. 

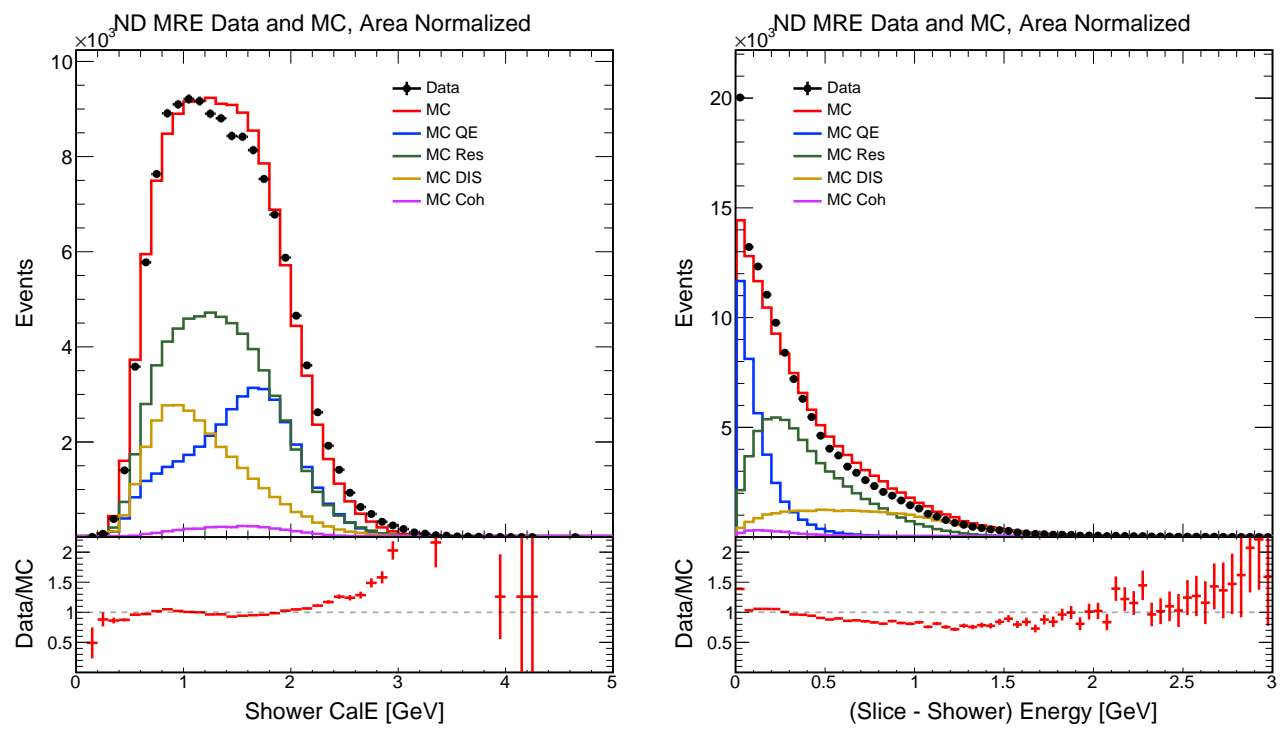

(a) Reconstructed primary shower energy (b) Reconstructed hadronic energy of MRE of MRE data and MC data and MC
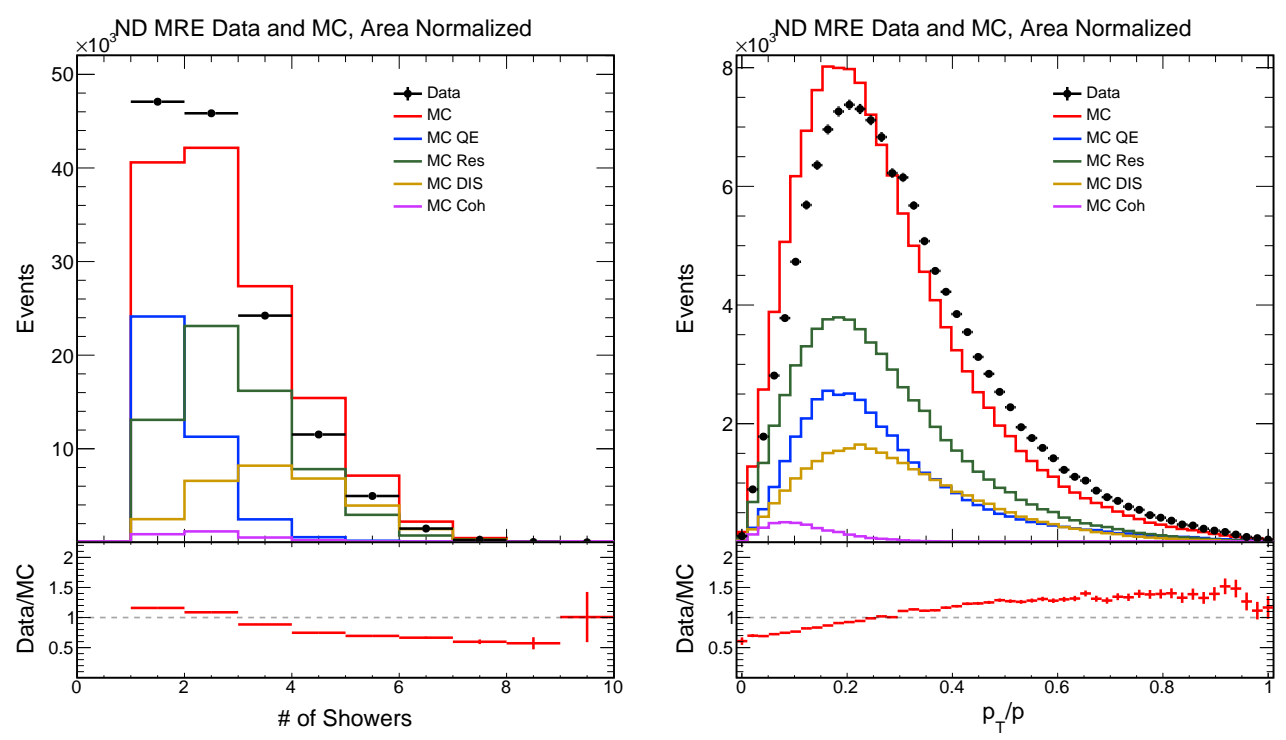

(c) Shower multiplicity of MRE data and (d) Transverse momentum fraction of MRE $\mathrm{MC}$ data and $\mathrm{MC}$

Figure 10.5: Comparison of MRE $\nu_{e}$ preselected events in ND data and MC. MC is area normalized to the data to emphasize the shape difference. 
However, the LID selection efficiency on MRE data (35.5\%) is higher by $17.5 \%$ than in $\mathrm{MC}(29.3 \%)$. This can be seen in figure 10.4a. Note that the LID distributions for MRE data and MC show similar features to those in the standard data and MC (see figure $9.4 \mathrm{a}$ ); in the intermediate region of LID, there are fewer data events than in MC, while in the LID signal region, the opposite is true. Figure 10.4b shows that the reconstructed neutrino energy of MRE events in MC is systematically higher than the MRE data. The hadronic energy distribution (figure 10.5b) also exhibits the same features as the standard data and MC disagreement. This points to the possibility that the data and MC difference in MRE and the standard events have a common source the incorrect modeling of the hadronic system in simulated neutrino interactions. This notion is explored further in the next section.

\subsubsection{MRE with Interaction Mode Scaling}

In the previous chapter, section 9.2.2, it was observed that the hadronic energy discrepancy in the ND data and MC could be arising from simulating an excess of deep-inelastic (DIS) interactions and not enough quasi-elastics (QE). A fit to hadronic energy distribution of $\nu_{e}$ preselected events for scales of interaction modes gave a factor of 1.86 for QE, 0.97 for resonance and 0.74 for DIS. Applying these scale factors to the MC improved the agreement with data of many distributions. These scale factors have now been applied to the MRE events in simulation and the results are in figure 10.6. 

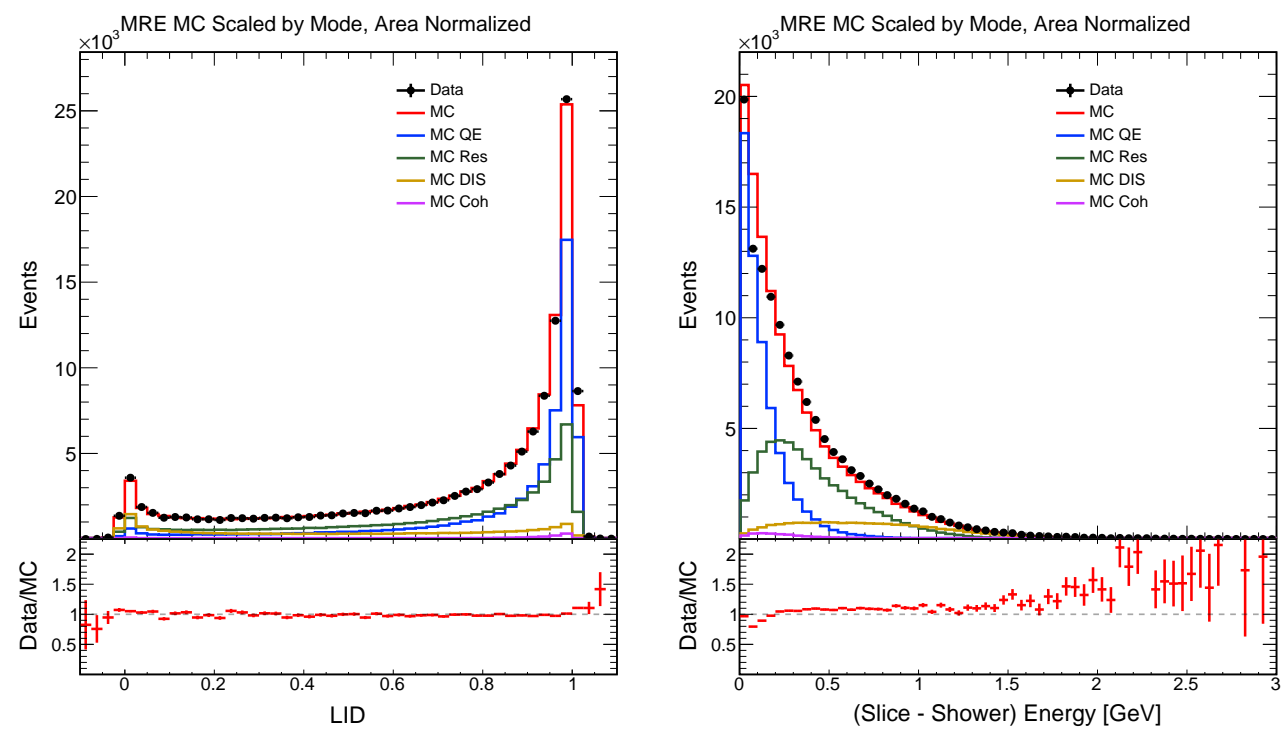

(a) LID distribution of MRE events, MC

(b) Hadronic energy in MRE events, MC with interaction mode scaling with interaction mode scaling
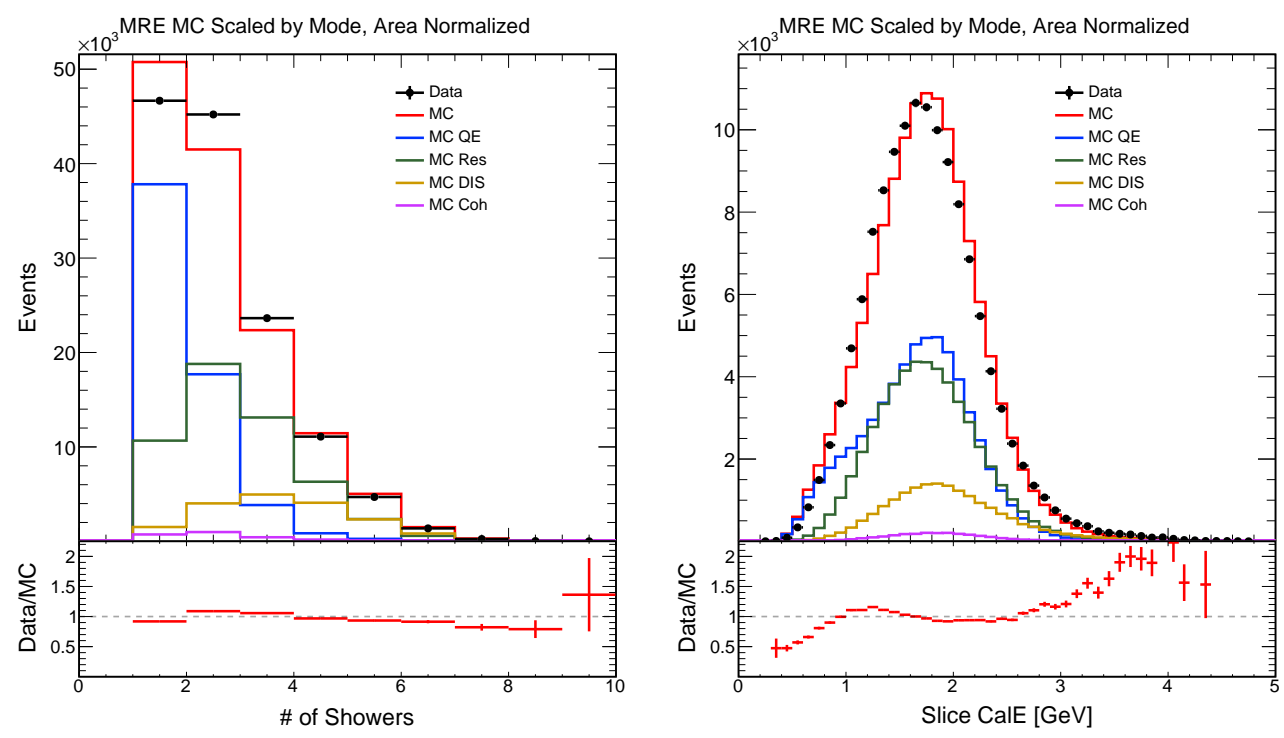

(c) Shower multiplicity in MRE events, MC (d) Neutrino energy of MRE events, MC with interaction mode scaling with interaction mode scaling

Figure 10.6: MRE in MC with interaction mode scaled by factor from 9.6 
Figure 10.6a shows that after scaling the interaction modes in MC with factors from table 9.6, the LID shapes of MRE data and MC agree remarkably well. The other distributions in 10.6 also show some minor improvements. The MRE selection efficiency in scaled MC is $34.9 \%$, which is only different by $1.7 \%$ from that in data of $35.5 \%$, compared to the difference of $17.5 \%$ without the scaling.

\subsection{MRE Correction On Signal Prediction}

There is strong evidence from MRE events in ND that the $\nu_{e}$ selection efficiency is much higher in data than in MC. Furthermore, the effect appears to arise from detector response to hadrons or the physics model of hadrons in neutrino interactions, or a combination of both. Since the neutrino simulator is configured the same way for both, near and far detector simulations, these issues must also be present in the far detector simulation. It is therefore necessary to correct the $\nu_{e}$ prediction in the far detector to account for this higher efficiency. However, the correction may not be applied directly. The vastly different sizes of the near and far detectors impose different kinematic constraints on the selected events which we will need to correct for. Moreover, the MRE events are constructed from $\nu_{\mu}$ selected events, so they are already subjected to a selection which has its own biases in the kinematic phase-space that aren't necessarily the same as those of the $\nu_{e}$ selection. The scheme that has been developed to circumvent these issues is described below.

\subsubsection{Efficiency by Shower and Hadronic Energy}

The hadronic showers in the ND data and simulation are known to disagree in energy scale and multiplicity and are the likely cause of the difference in behavior of LID in the standard data and MC. The LID $\nu_{e}$ identifier uses the most energetic shower in the event to measure the likelihood of the electron hypothesis. Therefore, two of the important kinematic variables that impact the response of LID are the energy of the 
primary shower and the hadronic energy component of the event.

The MRE selection efficiency in the near detector data and simulation is computed in the $2 \mathrm{D}$ space of leading shower energy and the hadronic energy in the event. Efficiency here is defined as the fraction of preselected MRE events (figures 10.7a and 10.7b) that pass the LID cut (figures 10.7c and 10.7d). These efficiency maps for data and MC are shown in figures $10.7 \mathrm{e}$ and $10.7 \mathrm{f}$. The sparsely populated bins are combined together to reduce statistical fluctuations. 

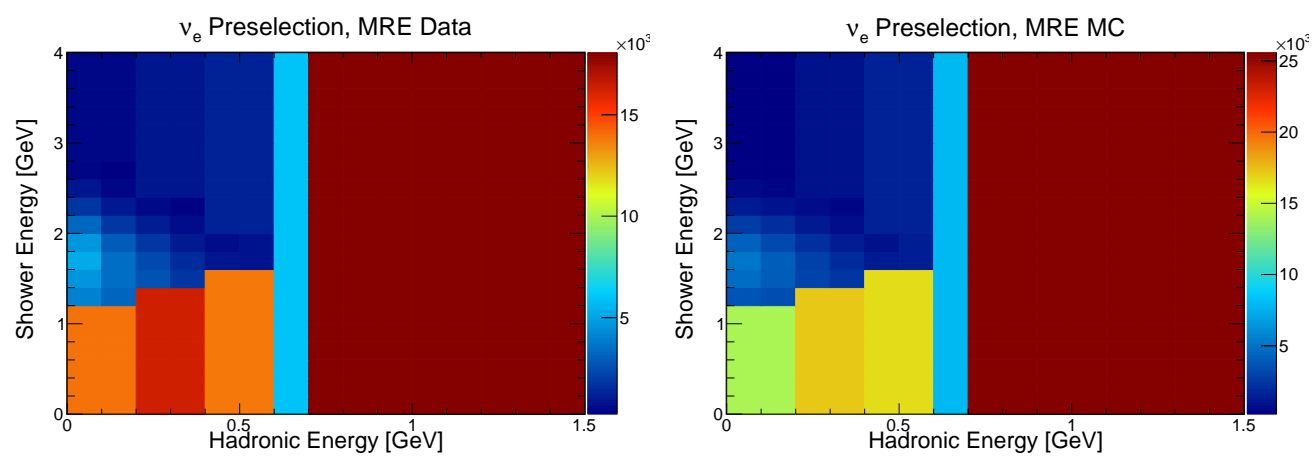

(a) MRE Events that pass $\nu_{e}$ preselection (b) MRE Events that pass $\nu_{e}$ preselection in data in $\mathrm{MC}$
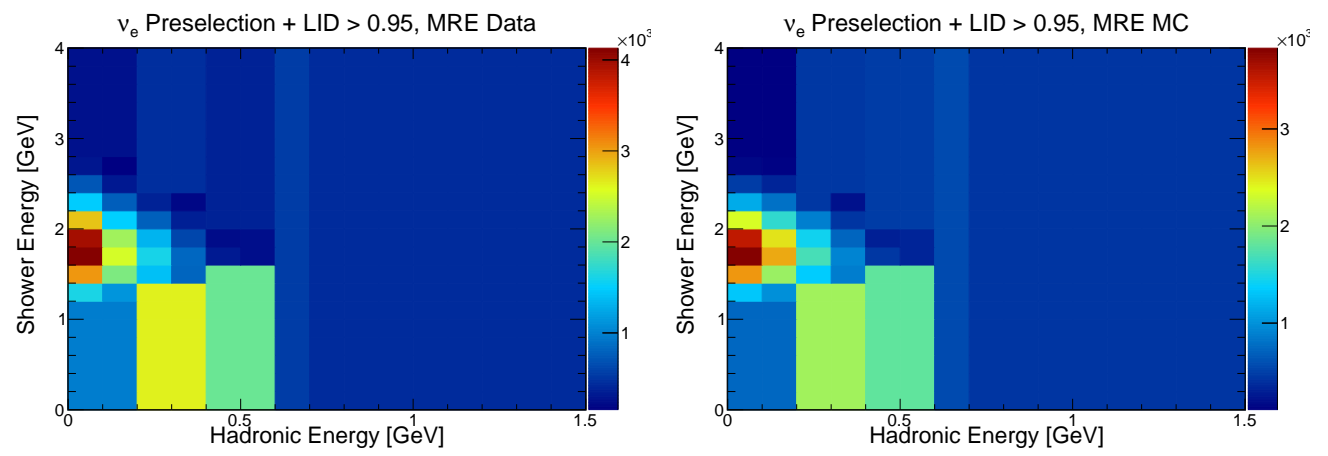

(c) MRE Events that pass LID in addition (d) MRE events that pass LID in addition to $\nu_{e}$ preselection in data to $\nu_{e}$ preselection in $\mathrm{MC}$

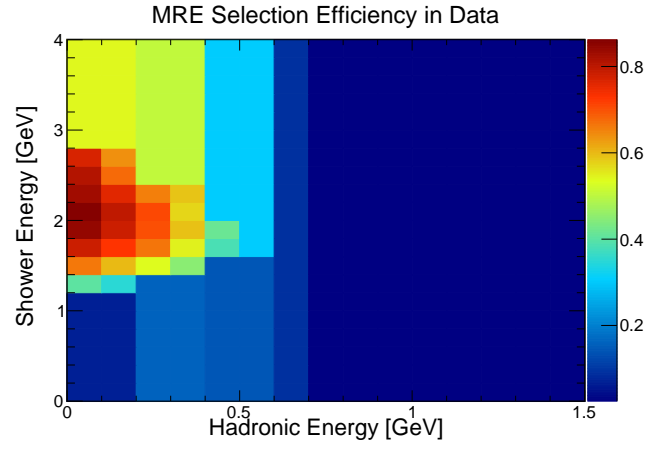

(e) MRE selection efficiency in data

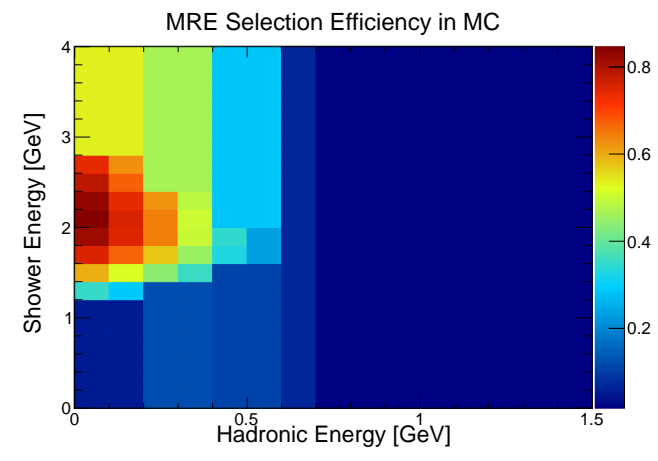

(f) MRE selection efficiency in MC

Figure 10.7: MRE events in data and MC after preseletion and LID cuts are used to construct a LID efficiency map in the primary shower and hadronic energy space. 


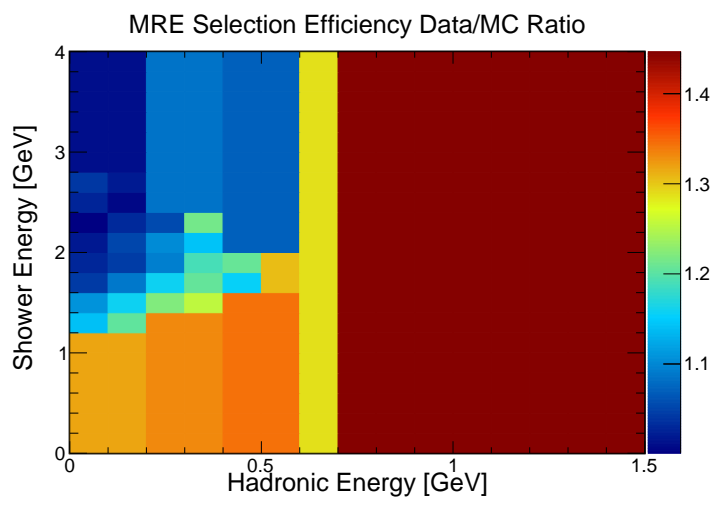

Figure 10.8: Efficiency ratio of MRE selection efficiency in data and simulation

The data to MC ratio of efficiencies in this 2D space (figure 10.8) is then used to reweight the far detector signal events that pass all $\nu_{e}$ selection criteria to give a corrected prediction. This procedure is, by design, insensitive to the physics model of shower and hadronic energy. The efficiency ratio shows that the MRE selection efficiency in the data and simulation are comparable when the leading shower is energetic $(\sim 2$ $\mathrm{GeV}$ ) and there is little hadronic energy in the event. As the balance shifts towards higher fraction of hadronic energy in the event, the selection efficiency in data exceeds that in the MC. 

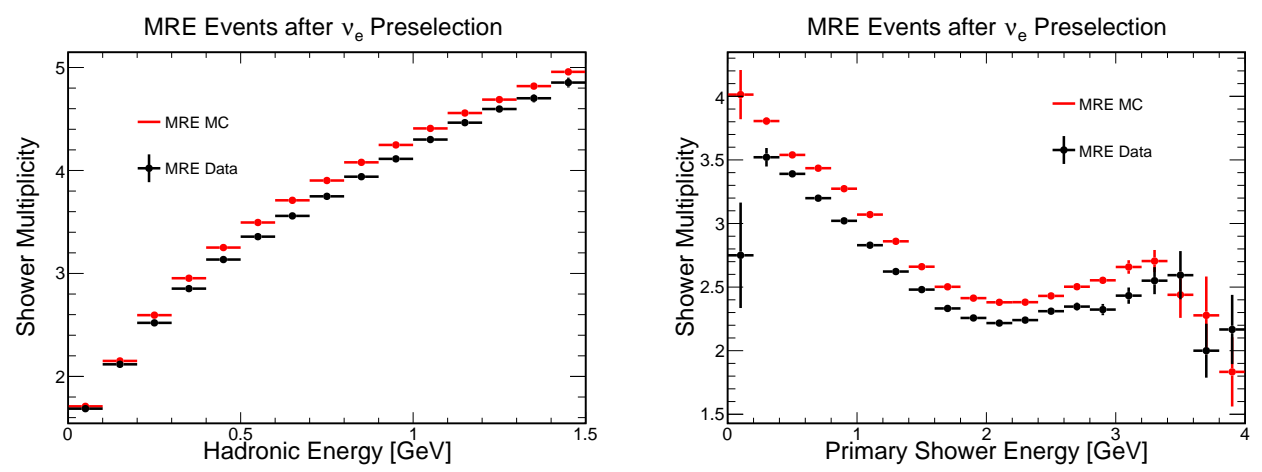

(a) Shower multiplicity as a function of (b) Shower multiplicity as a function of prihadronic energy in MRE events mary shower energy in MRE events

Figure 10.9: Shower multiplicity as a function of primary shower and hadronic energy in MRE events. MRE MC on average has higher shower multiplicity than data uniformly across a wide range of hadronic and leading shower energies.
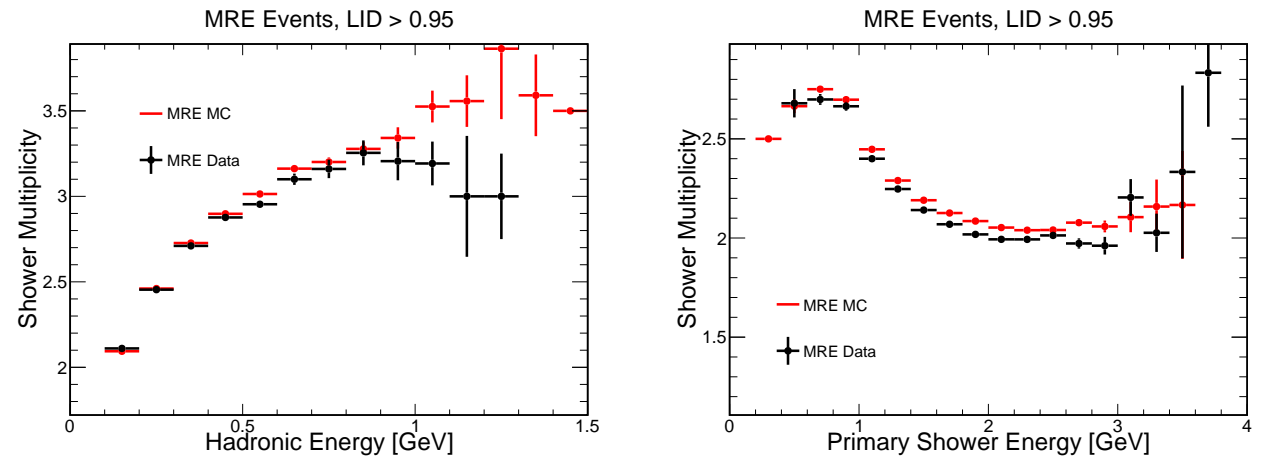

(a) Shower multiplicity as a function of (b) Shower multiplicity as a function of prihadronic energy in MRE events mary shower energy in MRE events

Figure 10.10: Shower multiplicity as a function of primary shower and hadronic energy in MRE events. MRE MC on average has higher shower multiplicity than data uniformly across a wide range of hadronic and leading shower energies.

Recall that the MRE data has a significantly higher proportion of events with low 
multiplicity (1 or 2 reconstructed shower in event) as shown in 10.5c. Figure 10.9 further shows that $\mathrm{MC}$ on average has a higher multiplicity than data uniformly across a wide range of hadronic and shower energies. The average multiplicity and the difference in average multiplicity in data and simulation are smaller after applying the LID cut (figure 10.10) indicating that LID preferentially selects lower multiplicity events. If the same amount of hadronic energy is split across many rather than a few particles, the overlap of a hadron track or shower with the primary shower becomes more likely (see figure 10.11). This distorts the measured plane $d E / d x$ from the values typical of an electron shower, making it harder to correctly identify them as electrons. This is at least one way of interpreting the observed selection efficiency difference between MRE data and MC.
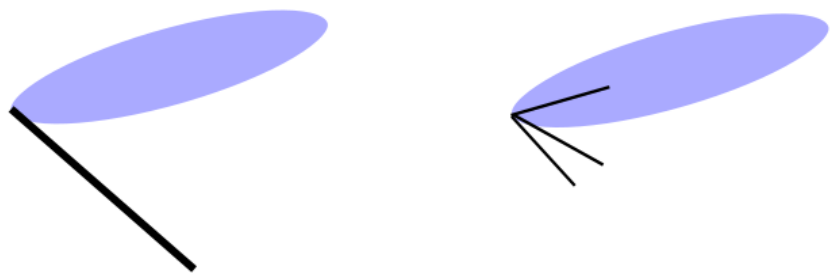

Figure 10.11: A cartoon of hadronic showers (black lines) of same energy but different multiplicity. The higher multiplicity shower is more likely to overlap with the EM shower (blue)

The application of this correction to the extrapolated far detector prediction is discussed in chapter 11. Here, in figure 10.12, the correction is simply applied to the far detector simulation to illustrate the size of the effect. The number of expected signal events in the FD simulation for the dataset analyzed in this document is 4.37. Upon correction due to MRE, the expectation rises to 5.06 events, an increase of $15.7 \%$. 

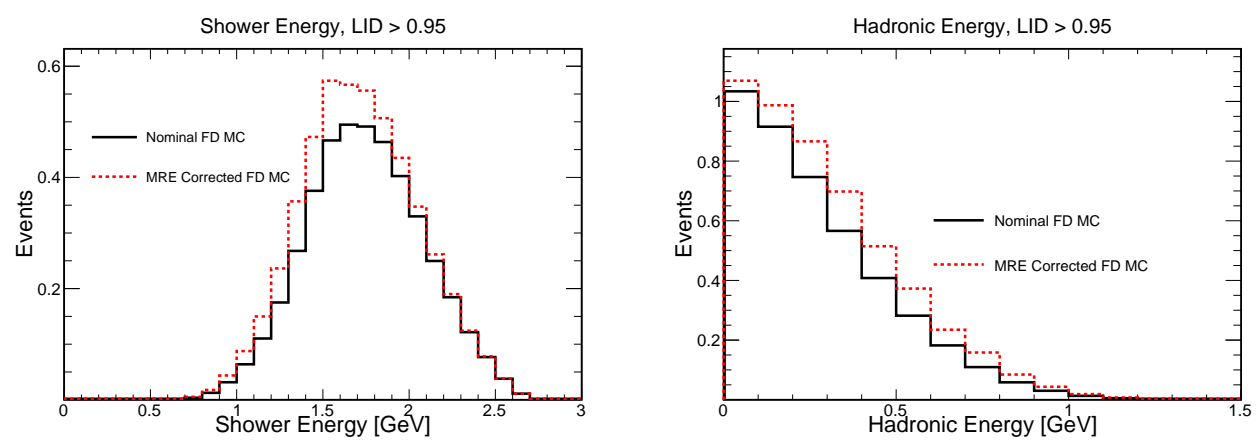

(a) Shower energy of $\nu_{e}$ signal events in FD (b) Hadronic of $\nu_{e}$ signal events in FD simsimulation ulation

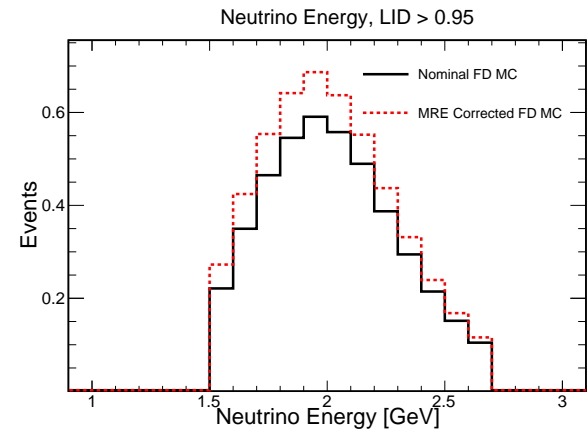

(c) Neutrino energy of $\nu_{e}$ signal events in

FD simulation

Figure 10.12: Energy distributions of $\nu_{e}$ selected signal events in FD simulation before and after MRE efficiency correction has been applied

\section{Summary}

MRE events are constructed by removing muon tracks from $\nu_{\mu} \mathrm{CC}$ interactions in ND data and MC and replacing them with simulated electrons with same kinematics (origin, direction and energy). Therefore, these events combine electron showers that are wellsimulated with hadronic showers in data, that have much larger uncertainties on them, to give a real-world-like sample of $\nu_{e} \mathrm{CC}$ interactions. MRE samples have been used 
to study the impact of the observed hadronic shower difference in ND data and MC on $\nu_{e}$ selection efficiency. The $\nu_{e}$ PID selection efficiency of MRE data is higher than that in $\mathrm{MC}$ by $17.5 \%$. This difference appears to arise from hadronic energy and particle multiplicity differences in data and $\mathrm{MC}$, an effect that we also expect to be present in the FD simulation and data. Therefore, a scheme to use the MRE efficiency to correct for this difference has been presented in this chapter. The correction leads to a significant rise of $15.7 \%$ in the expected signal events in the FD based on simulation. The difference in signal that we expect for $\delta=\pi / 2$ and $\delta=\pi$ is about $\sim 16 \%$ in case of normal hierarchy and maximal mixing. So the MRE correction to efficiency is comparable to the size of physics effects that we would like to measure. Finding the source of this large difference between simulation and data is one of the most important problems to tackle before future analyses of $\mathrm{NO} \nu \mathrm{A}$ data.

The MRE correction of the final extrapolated signal prediction is presented in chapter 11 and the systematic error on the MRE correction is treated in chapter 12 . 


\section{Chapter 11}

\section{Decomposition and Extrapolation}

The near detector contains no $\nu_{\mu}$ to $\nu_{e}$ appearance signal, therefore all the events that pass $\nu_{e}$ selection represent background to the appearance signal. Decomposition is the process of estimating the fractions of $\mathrm{NC}, \nu_{\mu} \mathrm{CC}$ and beam $\nu_{e} \mathrm{CC}$ background interactions in the $\nu_{e}$ selected sample in the near detector. The purpose of extrapolation is to derive a far detector event rate prediction based on the observed event rates in different channels in the near detector. In simple terms, we use far over near ratio from simulation and correct it by the near detector data, to obtain a far detector estimate. The advantage of this approach is that many systematics that affect the two detectors in similar ways cancel out in the far over near ratio and results in a smaller error on the measurement. In this chapter we first discuss decomposition of the ND data, the method used for extrapolation of background and signal and then finally, the far detector prediction resulting from this exercise. 


\subsection{Decomposition}

\subsubsection{Proportional Decomposition}

In this decomposition method, the simulation is trusted to correctly simulate the relative rates of the $\mathrm{NC}, \nu_{\mu} \mathrm{CC}$ and beam nue $\mathrm{CC}$ interaction rates in the $\nu_{e}$ selected sample in the ND, but relies on data for the absolute normalization. From the final row of table 9.2, after applying all $\nu_{e}$ selection cuts, MC has 2471 events, which contains $31.7 \% \mathrm{NC}$, $16 \% \nu_{\mu} \mathrm{CC}$ and $52.3 \%$ beam $\nu_{e} \mathrm{CC}$ interactions. In the data, however, 2579 survive $\nu_{e}$ selection, $4.4 \%$ higher than MC. It is assumed that MC scale is uniformly low and all the three interaction types are scaled up by $4.4 \%$ so that the MC is consistent with data in norm. This does not change the fraction of $\mathrm{NC}, \nu_{\mu} \mathrm{CC}$ and beam $\nu_{e} \mathrm{CC}$ interactions in the ND MC.

\subsubsection{Decomposition, Upon Scaling by Interaction Type}

In section 9.2.1, the $\mathrm{NC}$ and $\nu_{\mu} \mathrm{CC}$ scale factors were derived from fitting the reconstructed neutrino energy of ND events in the intermediate LID region. These scale factors were then used to fit for the scale of beam $\nu_{e} \mathrm{CC}$ interactions in the neutrino energy distribution of events in the high intermediate region of LID. The fit scales are 1.12 for $\mathrm{NC}, 0.46$ for beam $\nu_{e} \mathrm{CC}$ and 1.14 for beam $\nu_{e} \mathrm{CC}$. This changes the proportion of interactions in the ND MC to $34.6 \% \mathrm{NC}, 7.2 \% \nu_{\mu} \mathrm{CC}$ and $58.2 \%$ beam $\nu_{e} \mathrm{CC}$. The $\mathrm{MC}$ is again normalized to data to give the decomposed background counts.

\subsubsection{Decomposition, Upon Scaling by Interaction Mode}

In section 9.2.2, the relative proportions of QE, resonance and DIS interaction modes that best describe the data were found by fitting the hadronic energy distribution. The quasi-elastic interactions that pass $\nu_{e}$ selection in the ND are usually due to charged current scattering of beam $\nu_{e}$ whereas the $\nu_{\mu}$ CC's that are a background to $\nu_{e}$ appearance are usually deep-inelastic. The neutral currents that pass $\nu_{e}$ selection are mostly 
deep-inelastic or coherent scatterings. Scaling the interaction modes by the derived factors changes the relative proportion of ND MC such that it contains $24.4 \% \mathrm{NC}, 11.5 \%$ $\nu_{\mu} \mathrm{CC}$ and $64.1 \%$ beam $\nu_{e} \mathrm{CC}$.

\subsubsection{Summary of Decomposition}

Table 11.1 summarizes the result of the decomposition methods discussed above. For this analysis, the result from the proportional decomposition will be used for the background estimate. The results of decomposition from scaling by typing and mode will be used to estimate the error on the background prediction.

\begin{tabular}{l|lll}
\hline Method & NC & $\nu_{\mu} \mathrm{CC}$ & Beam $\nu_{e} \mathrm{CC}$ \\
\hline ND MC & $783(31.7 \%)$ & $396(16 \%)$ & $1292(52.3 \%)$ \\
Proportional & $817(31.7 \%)$ & $413(16 \%)$ & $1348(52.3 \%)$ \\
Proportional, Scaled by type & $892(34.6 \%)$ & $187(7.3 \%)$ & $1500(58.2 \%)$ \\
Proportional, Scaled by mode & $629(24.4 \%)$ & $300(11.6 \%)$ & $1650(64.0 \%)$ \\
\hline
\end{tabular}

Table 11.1: Result of decomposition of $\nu_{e}$ selected data in the near detector

\subsection{Background Extrapolation}

The beam background components predicted by extrapolation are neutral current, $\nu_{\mu}$ $\mathrm{CC}$ and intrinsic beam $\nu_{e}$ CC. $\nu_{\tau} \mathrm{CC}$ background is known to be very small, so we have opted to estimate it directly by using far detector simulation, rather than extrapolating it. We apply the $\nu_{e}$ selection cuts to the data and simulation in the near detector. The reconstructed energy spectrum of the selected events is decomposed into the three different components- NC, beam $\nu_{e}$ (and beam $\bar{\nu}_{e}$ ) CC and $\nu_{\mu}\left(\right.$ and $\bar{\nu}_{\mu}$ ) CC. The far detector estimate of these background components is obtained by scaling the $\nu_{e}$ selected background in each channel in the far detector simulation by the data-MC ratio of that 
component in the near detector, and weighting by the oscillation probability.

$$
\left.F\left(E_{j}^{r e c o}\right)\right|_{\text {pred }}=\left.F\left(E_{j}^{r e c o}\right)\right|_{\text {sim }} \times \frac{\left.N\left(E_{j}^{r e c o}\right)\right|_{\text {data }}}{\left.N\left(E_{j}^{r e c o}\right)\right|_{\text {sim }}} \times P\left(\nu_{x} \rightarrow \nu_{y}\right)
$$

where $j$ is a bin in reconstructed neutrino energy, $F$ denotes the event counts in FD and $N$ in ND. The oscillation weight for neutral currents is 1 . The near detector data and MC spectra and the far detector simulated and predicted spectra for the three beam backgrounds are shown in figure 11.1. 

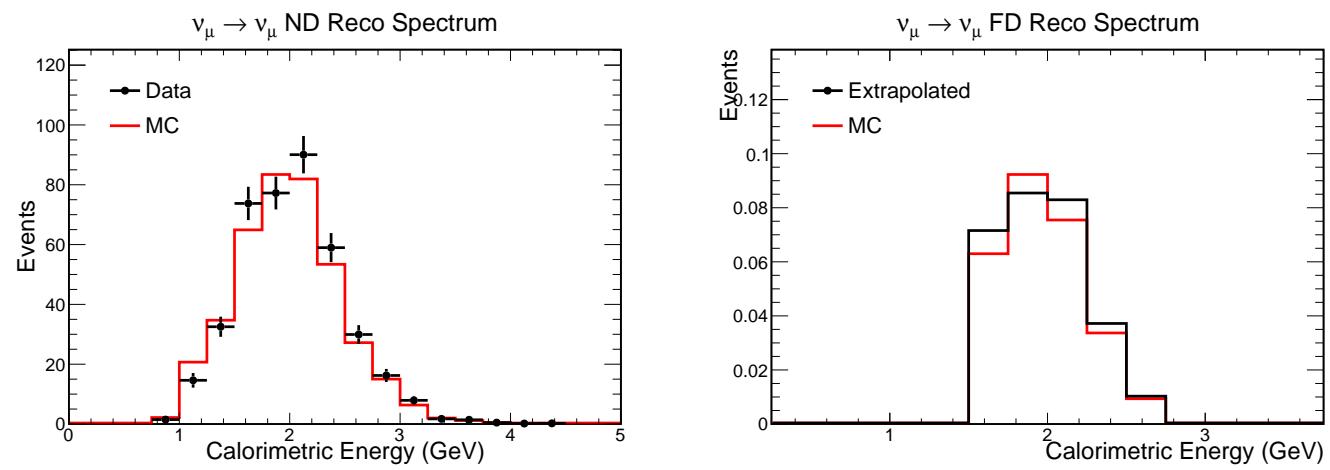

(a) Reconstructed energy spectrum of $\nu_{\mu} \mathrm{CC}$

(b) Reconstructed energy spectrum of $\nu_{\mu} \mathrm{CC}$ background in ND background in FD
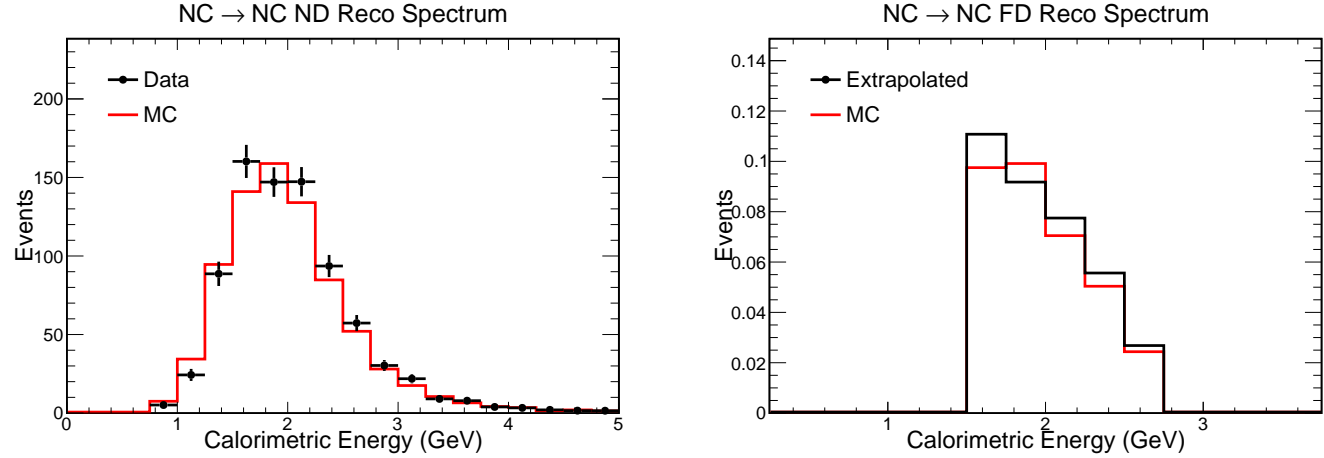

(c) Reconstructed energy spectrum of $\mathrm{NC}$ background in ND
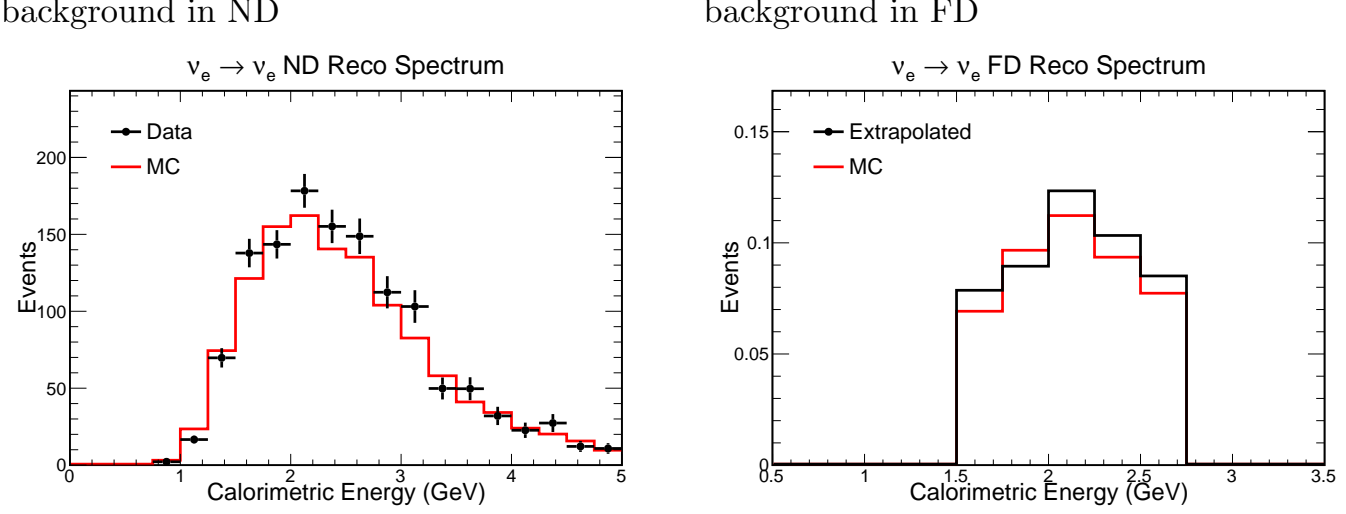

(e) Reconstructed energy spectrum of beam $\nu_{e}$ (f) Reconstructed energy spectrum of beam $\nu_{e}$ CC background in ND $\mathrm{CC}$ background in FD

Figure 11.1: Reconstructed energy of $\mathrm{NC}$ and $\nu_{\mu} \mathrm{CC}$ backgrounds in the near detector and extrapolated spectra in the far detector. 


\subsection{Signal Extrapolation}

The $\nu_{\mu}$ to $\nu_{e}$ appearance signal expected in the far detector must be constrained by the flux of $\nu_{\mu}$ 's observed in the near detector. In the near detector, for selection of $\nu_{e}$ background events, we ignore the muon catcher, but to contain $\nu_{\mu} \mathrm{CC}$ interactions that are representative of the oscillating population, ie with energy around $2 \mathrm{GeV}$, muon catcher is necessary to range out muons. Due to this, we can not use the simple calorimetric energy reconstruction for $\nu_{\mu}$ 's as we use in the $\nu_{e}$ analysis, as that does not properly account for energy loss in steel. Instead, we use the $\nu_{\mu}$ energy reconstruction that is used in the disappearance analysis where the muon energy estimated from range is summed with all other energy in the slice corrected for loss in dead material etc (see section 7.5). Since the energy of the $\nu_{\mu} \mathrm{CC}$ interactions that the $\nu_{e}$ signal is extrapolated from is reconstructed in a different way and has a different resolution, the signal extrapolation is done in true neutrino energy.

We select a pure sample of $\nu_{\mu}$ CC interactions in the near detector using ReMID, the $\nu_{\mu}$ CC PID (see section 7.5). $\nu_{\mu}$ selection cuts in the ND are listed is table 9.3. The selection applied to the simulation and data are identical. Using the simulation, a matrix $M^{\mu}$ is constructed to map a reconstructed $\nu_{\mu}$ CC energy to the true $\nu_{\mu}$ energy in the form of a $2 \mathrm{D}$ histogram. Using this matrix, the observed $\nu_{\mu}$ energy spectrum in the near detector data is converted into an underlying true $\nu_{\mu}$ energy distribution as follows:

$$
N\left(E_{i}^{\text {true }}\right)_{\text {data }}=\sum_{j} M_{i j}^{\mu} \frac{N\left(E_{j, \mu}^{r e c o}\right)_{\text {data }}}{N\left(E_{j, \mu}^{r e c o}\right)_{\text {sim }}}
$$

That is, the row corresponding to a true energy is corrected by the data/MC ratio in bins of reconstructed energy, and summed to get an event count at that true energy in data. The $\nu_{\mu} \mathrm{CC}$ energy distributions in the near detector data and $\mathrm{MC}$, the reconstructed to true energy conversion matrix, and the estimated true energy distributions of ND $\nu_{\mu}$ events are shown in figure 11.2 
A similar true $\leftrightarrow$ reconstructed energy matrix, $M^{e}$ is constructed using the far detector simulation, to convert a true electron neutrino energy spectrum to a reconstructed one. This matrix is corrected by the true ND data and ND MC $\nu_{\mu}$ energy distributions.

$$
M_{i j, \text { corr }}^{e}=M_{i j}^{e} \times \frac{N\left(E_{i}^{\text {true }}\right)_{\text {data }}}{N\left(E_{i}^{\text {true }}\right)_{\text {sim }}}
$$

Finally, the corrected true $\leftrightarrow$ reconstructed $\nu_{e}$ energy matrix is projected, and weighted by $\nu_{\mu}$ to $\nu_{e}$ oscillation probability, to obtain a prediction of the $\nu_{e} \mathrm{CC}$ signal.

$$
F\left(E_{j, e}^{r e c o}\right)_{\text {pred }}=\sum_{i} M_{i j, c o r r}^{e} P_{\nu_{\mu} \rightarrow \nu_{e}}\left(E_{i}^{\text {true }}\right)
$$

Plots that show this progression into a predicted spectrum are shown in figure 11.3. 

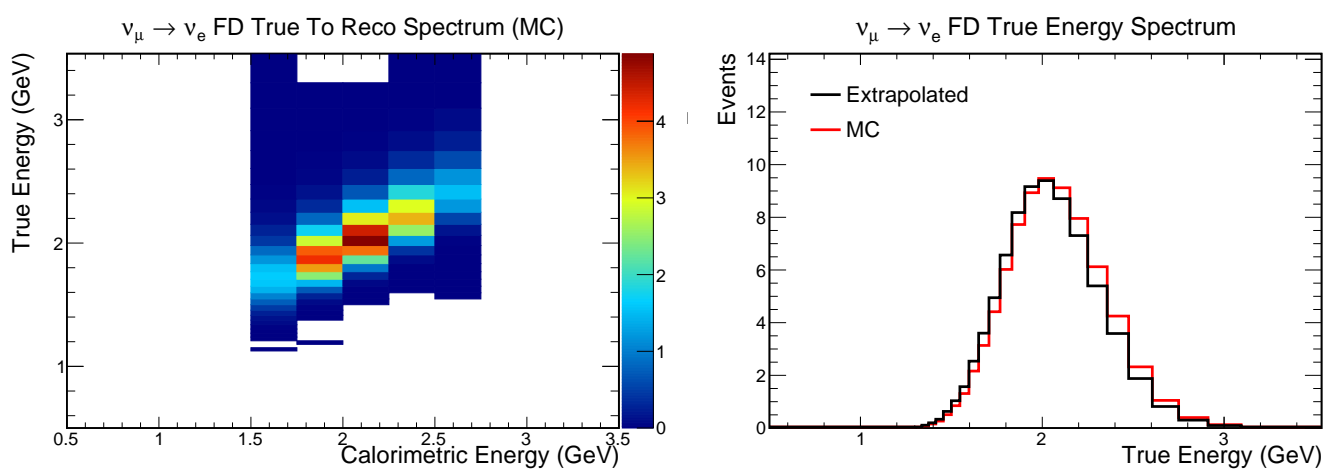

(a) Reconstructed to true energy map of $\nu_{e}$ (b) True energy of $\nu_{e} \mathrm{CC}$ selected events in $\mathrm{CC}$ selected events in FD MC

FD corrected by the ND distributions.

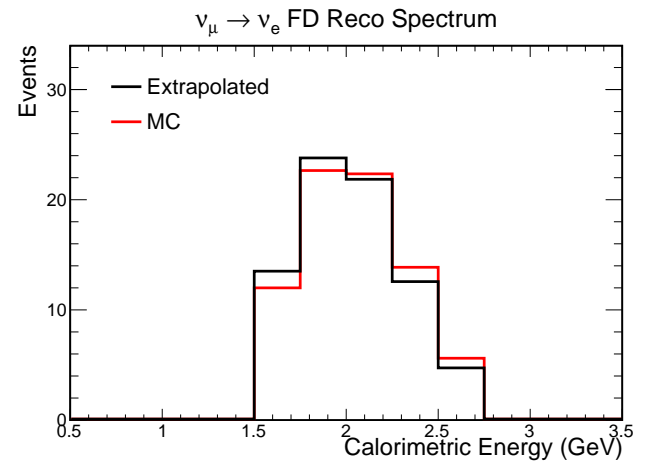

(c) Reconstructed energy distribution of the predicted $\nu_{e}$ appearance signal in FD.

Figure 11.3: Extraction of a predicted $\nu_{e}$ reconstructed energy spectrum from FD true to reconstructed $\nu_{e}$ energy map and ND true energy spectra for data and MC.

As shown in 11.2, the reconstructed energy of $\nu_{\mu}$ CC interactions in the ND data peaks about $5 \%$ lower than in the ND MC $(1.77 \mathrm{GeV}$ in data compared with $1.87 \mathrm{GeV}$ in $\mathrm{MC}$ ). The reconstructed energy in data maps to a lower true energy (figure 11.4b) and this directly impacts the oscillation probability and the predicted $\nu_{e}$ appearance signal energy spectrum. If the mode scale factors discussed in section 9.2 .2 are applied to the $\nu_{\mu}$ sample, an improvement in the data-MC agreement in the neutrino energy is observed (figure 11.4). However, the disagreement in ND data and MC in $\nu_{\mu}$ energy has 
little impact on the total number of predicted signal events $(<0.2 \%)$, and therefore, is not pursued any further in this document.

\subsection{Results of Extrapolation}

The prediction of signal and different beam background components in the far detector for this analysis are shown in table 11.2 for simple oscillations (see section 5.3). The predictions for the two extreme oscillation combinations of hierarchies and $\delta$ are in table 11.3. The estimates are based on real-conditions far detector simulation and near detector data and simulation.

Since the near detector data has a $\sim 4 \%$ excess over simulation after applying $\nu_{e}$ selection, the background event counts from extrapolation are higher than those from the far detector simulation. There are $4.7 \%$ more $\nu_{\mu} \mathrm{CC}$ interaction in data than in MC, therefore the signal expectation also rises on extrapolation. The extrapolated signal prediction is $1.1 \%$ higher than the FD simulation prediction. The MRE correction, discussed in detail in section 10.3, produces a much larger effect of increasing the extrapolated signal from 4.41 to 5.1 , ie by $15.8 \%$.

To conclude this chapter, in the absence of $\nu_{\mu}$ to $\nu_{e}$ oscillation, we expect to observe about 1 event, while in the presence of oscillations, the selected event count is expected to be close to 6 events for simple oscillations. The expected signal changes significantly with different oscillation parameters, but the background is relatively stable against such changes. The next chapter discusses the errors on these estimates. 


\begin{tabular}{l|ccccccc}
\hline & $\nu_{e}$ Signal & Total BG & NC & $\nu_{\mu}$ CC & Beam $\nu_{e}$ CC & $\nu_{\tau}$ CC & Cosmics \\
\hline FD MC & 4.36 & 0.91 & 0.34 & 0.05 & 0.44 & 0.02 & 0.06 \\
FD MC with MRE & 5.06 & 0.91 & 0.34 & 0.05 & 0.44 & 0.02 & 0.06 \\
\hline FD Extrap & 4.41 & 0.97 & 0.36 & 0.05 & 0.47 & 0.02 & 0.06 \\
FD Extrap with MRE & 5.10 & 0.97 & 0.36 & 0.05 & 0.47 & 0.02 & 0.06 \\
\hline FD Extrap, by type & 5.10 & 1.04 & 0.41 & 0.02 & 0.51 & 0.02 & 0.06 \\
FD Extrap, by mode & 5.10 & 0.99 & 0.28 & 0.04 & 0.60 & 0.02 & 0.06 \\
\hline
\end{tabular}

Table 11.2: Extrapolated signal and background predictions for $\nu_{e}$ appearance analysis of NO $\nu$ A's first data using simple oscillations. The highlighted row is the main prediction. The first two rows are from the far detector simulation directly. The bottom two rows are from using different ways of decomposing the background spectrum in ND

\begin{tabular}{l|ccccccc}
\hline Osc. Parameters & $\nu_{e}$ Signal & Total BG & NC & $\nu_{\mu}$ CC & Beam $\nu_{e}$ CC & $\nu_{\tau}$ CC & Cosmics \\
\hline Simple Osc, Extrap & 5.10 & 0.97 & 0.36 & 0.05 & 0.47 & 0.02 & 0.06 \\
IH, $\delta=\pi / 2$, Extrap & 2.59 & 0.96 & 0.36 & 0.05 & 0.48 & 0.02 & 0.06 \\
NH, $\delta=3 \pi / 2$, Extrap & 6.57 & 0.94 & 0.36 & 0.05 & 0.47 & 0.02 & 0.06 \\
\hline
\end{tabular}

Table 11.3: Predictions with different oscillation scenarios. NH and IH refer to normal and inverted hierarchies, that is $\Delta m_{32}^{2}>0$ and $\Delta m_{32}^{2}<0$ cases respectively. It can be seen in figure 3.2, that the bottom two rows represent the two most extreme oscillation scenarios in $\mathrm{NO} \nu \mathrm{A}$. All the oscillations listed above are maximal, that is $\theta_{23}=\pi / 4$ 

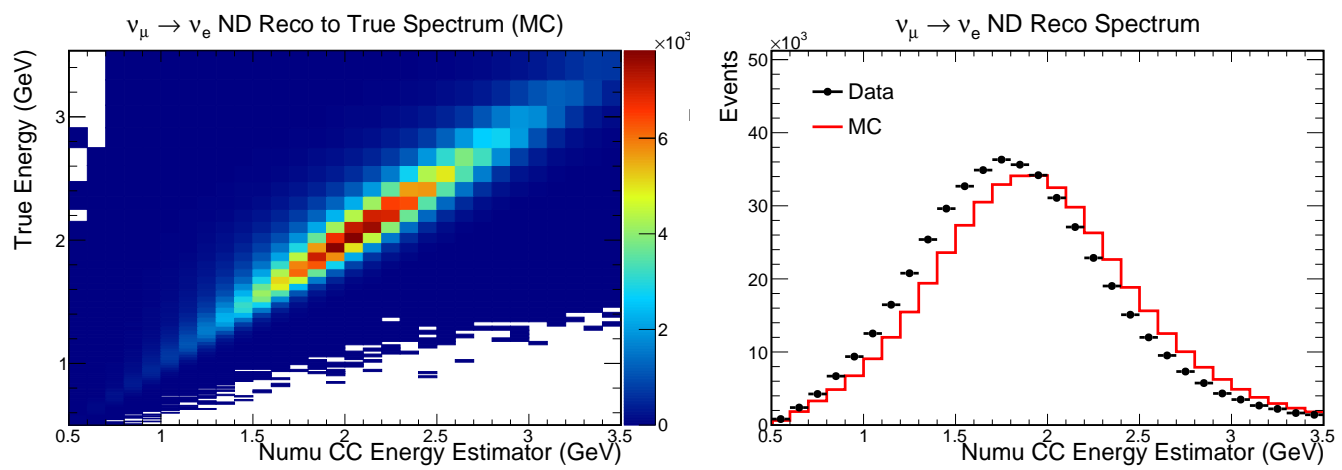

(a) Reconstructed to true energy map of $\nu_{\mu}$ (b) Reconstructed energy of $\nu_{\mu}$ CC selected $\mathrm{CC}$ selected events in ND MC events in ND for data and MC.

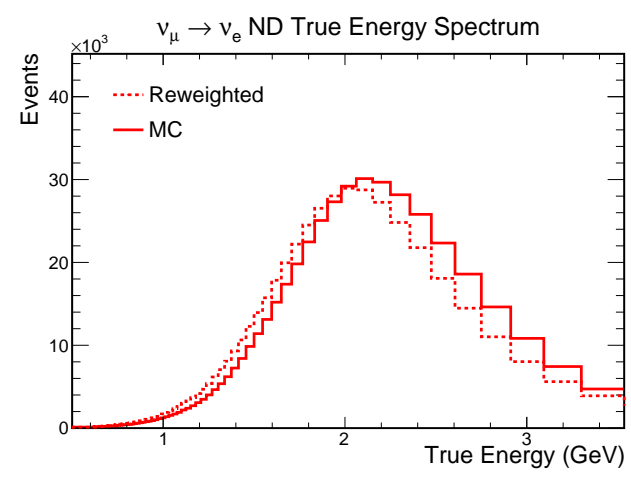

(c) True energy distribution for the observed reconstructed $\nu_{\mu}$ energy spectrum in ND data

Figure 11.2: Reconstructed energy to true energy conversion of the ND data $\nu_{\mu} \mathrm{CC}$ events 

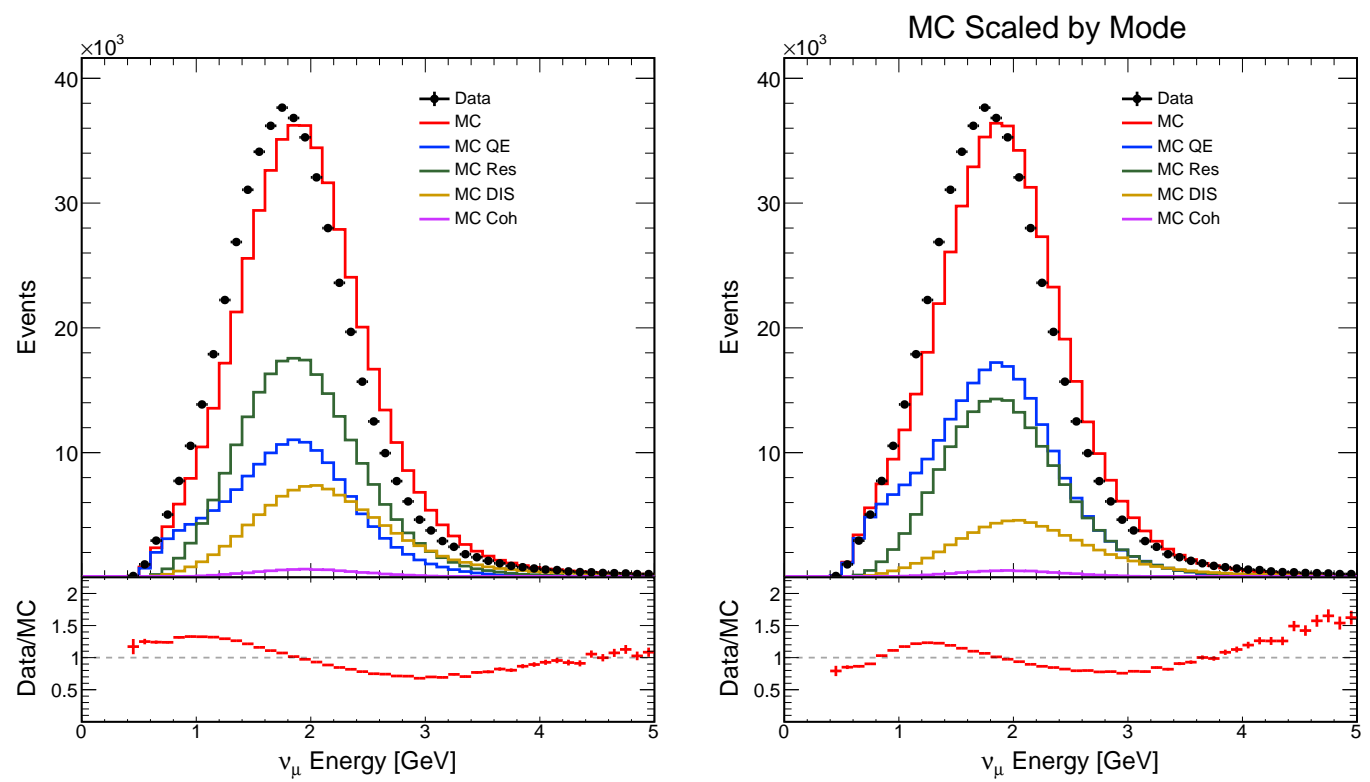

(a) Reconstructed energy of $\nu_{\mu}$ CC selected (b) Reconstructed energy of $\nu_{\mu}$ CC selected events in ND for data and MC events in ND for data and MC scaled by mode

Figure 11.4: Reconstructed energy of $\nu_{\mu}$ selected events in the ND, with and without mode scaling. 


\section{Chapter 12}

\section{Error Estimates}

The $\mathrm{NO} \nu \mathrm{A}$ near and far detectors are functionally identical and many systematic errors are reduced or canceled in the far over near extrapolation described in chapter 11 . To study the effect of systematic errors, we reweight the events, or generate systematically shifted far and near detector simulations, where possible, and derive new event rate predictions based on extrapolation using the shifted simulation. The errors considered for this analysis, their treatment and size of effect are considered in this chapter.

\subsection{Calibration Error}

The error due to miscalibration was expected to be one of the larger errors, because despite being identically built, the two detectors are calibrated separately. In the far detector, a large statistics sample of cosmic ray muon tracks that are used for calibration is easily available. Near detector is underground and the cosmic ray rate is significantly lower. The muons that reach the near detector have to be high in energy and are likely to emit bremsstrahlung radiation which can bias the calibration sample.

The effect of error due to miscalibration was assessed by deliberately miscalibrating the far and near detector simulations in a few different ways and finding the change in signal and background event counts. The different miscalibrations and their motivations 
are listed below:

\section{Energy Scale}

For this error, the calibrated energy scale in the simulation was changed by $\pm 5 \%$ of the nominal. The value of $5 \%$ is used because comparisons of the energy scale of some samples between data and MC show them to be different by $5 \%$. The most important of these for the $\nu_{e}$ analysis is the measurement of the energy spectrum of the decay electron of muon, or the Michel electron. The mean of the energy distribution of Michel electrons in data and MC in the near detector are $40.6 \mathrm{MeV}$ and $42.4 \mathrm{MeV}$, respectively, which are different by $\sim 5 \%$ (see figure 12.1a). The Michel energy scale has been tested in the FD as well, but the response is more complicated. The data is lower than MC at the end closer to the APD, and higher than the MC at the far end. This distance dependent response is considered next. The energy scale error could be present in both detectors, or only in one. The extrapolation was performed using the ND data and systematically shifted sets of near and far detector simulated samples, and the results were compared to the nominal extrapolation, to get an estimate of error due to miscalibrating both the detectors by the same amount. To estimate the relative error, systematically shifted ND simulation was used for extrapolation, instead of ND data, along with nominal ND and FD simulations.

\section{Response Slope}

In the far detector it has been found that the calibrated detector response behaves differently in data and $\mathrm{MC}$ along the length of a cell. A polynomial was fit to the data/MC ratio of response as a function of cell depth in the far detector as shown in figure 12.1b, and the resulting function was used to change the calibrated response as a function of the distance from readout in the far detector. This shifted FD MC was used in extrapolation in place of the nominal, to gauge the impact on predictions. 


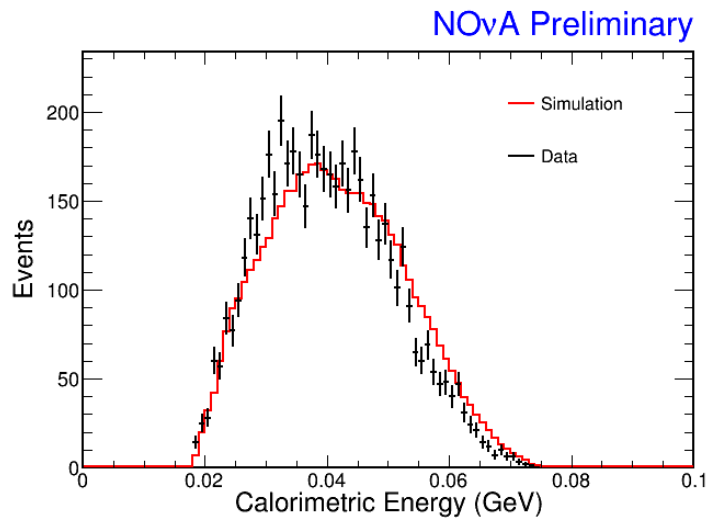

(a) Michel electron energy spectrum measured in (b) Difference in calibrated response as a data and ND MC.

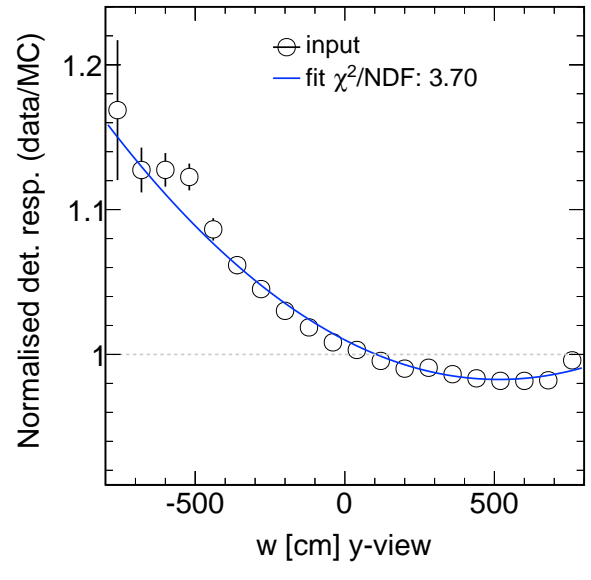

function of cell depth in FD cosmic data and MC.

Figure 12.1: Evidence of miscalibration in near and far detectors.

\section{Random Miscalibration}

The detector response changes and is calibrated cell by cell in the data. There is no such cell to cell variation in the simulation. To test the impact of this variation, a FD simulation sample was generated with randomly shifted response in each cell of the detector. The shifts have a Gaussian distribution with $1 \sigma=8 \%$, which far exceeds the observed variation seen in the far detector data, which is $\sim 1 \%$. Despite that, random miscalibration is demonstrated to be a very small effect on our selection.

\subsection{Interaction Model Uncertainties}

Improperly modeling particle interactions can not only cause us to rely on unrealistic models of neutrino interactions, but also skew the simulation of the neutrino beam spectrum and flux. These two effects are considered separately below. 


\begin{tabular}{l|lllll}
\hline & $\nu_{e}$ Signal $(\%)$ & Tot bg. $(\%)$ & $\nu_{\mu}$ CC $(\%)$ & NC $(\%)$ & Beam $\nu_{e}$ CC $(\%)$ \\
\hline Absolute & 5.98 & 0.76 & 8.45 & 10.81 & 10.89 \\
Relative & 4.36 & 3.72 & 12.07 & 15.65 & 6.39 \\
Random & -0.93 & -0.39 & 1.47 & 1.47 & 1.87 \\
Slope & 1.33 & 2.27 & 2.94 & 10.51 & 3.92 \\
\hline Total & 7.58 & 4.44 & 15.10 & 21.78 & 13.35 \\
\hline
\end{tabular}

Table 12.1: Calibration systematic errors

\subsubsection{Beam Flux Errors}

The model of the neutrino beam used is the same for both, the near and the far detectors, and the uncertainties in the model are expected to cancel to a large extent in the far over near ratio. The cancellation is not expected to be exact because the NuMI beamline is a line source at the location of the near detector, but it is effectively a point source for the far detector.

The beam uncertainties relate to the uncertainties in the operating conditions of the beam such as the horn and target positions, horn current, etc and these are grouped under beam transport errors. The other, bigger, source of error is the modeling of pion and kaon production rates at the target and their spectra, the uncertainties due to which are grouped under hadron production errors.

To estimate the errors from these sources, the beam transport and hadron production variables were changed by $1 \sigma$ from their central value and alternative near and far beam flux simulations were produced. The ratios of varied to nominal fluxes in bins of true neutrino energy were used to reweight the $\nu_{e}$ selected events. The uncertainty on a single detector is obtained by adding in quadrature the variations in event counts corresponding to variations in beam parameters. Figure 12.2 shows the size of beam systematic errors on the near detector energy and LID spectra. 
The uncertainty on the prediction using two detectors was estimated by extrapolating the reweighted spectra and measuring the percentage difference with respect to the nominal. The variations were then added in quadrature. The breakdown of uncertainty due to each beam parameter can be found in [43]. The overall uncertainty due to beam parameters on the extrapolated $\nu_{e}$ signal is found to be $1.06 \%$ and on background, $3.18 \%$.
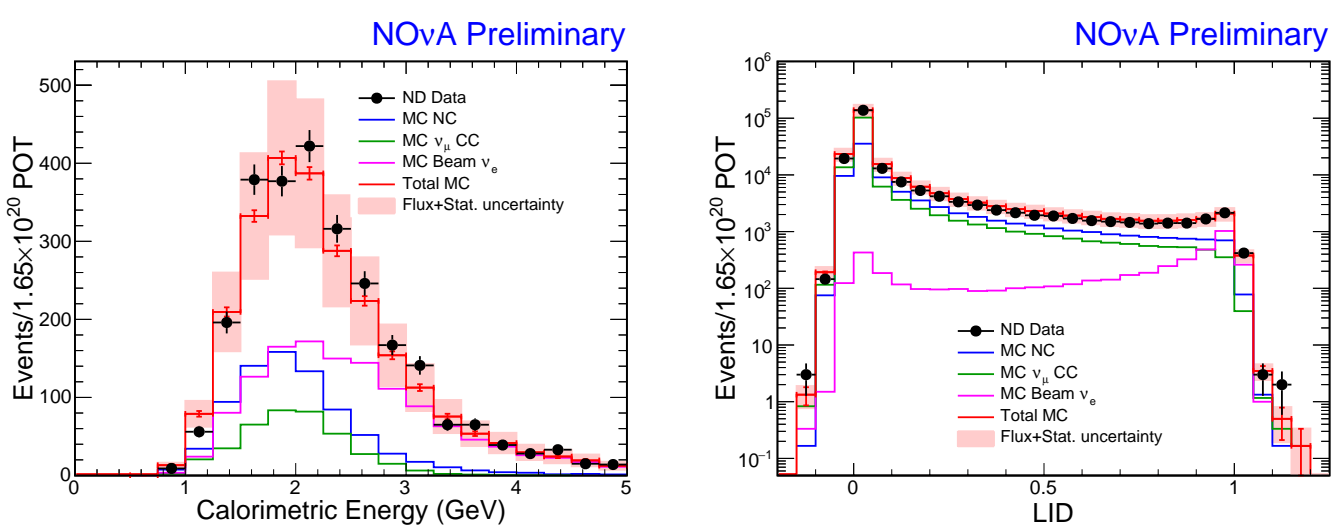

Figure 12.2: Near detector energy spectrum after all $\nu_{e}$ selection and LID spectrum after preslection. The error band consists of beam systematic errors.

\subsubsection{Neutrino Interaction Model Uncertainty}

Uncertainties in neutrino interaction modeling do not cancel exactly, for the same reason as the beam uncertainties. In addition, the $\nu_{e}$ appearance signal is extrapolated based on the observation of $\nu_{\mu} \mathrm{CC}$ energy spectrum in the near detector. A modeling error in one does not directly relate to the other because $\nu_{\mu} \mathrm{CC}$ selection and $\nu_{e} \mathrm{CC}$ selection have different efficiencies for different interaction modes.

The GENIE neutrino event generator provides a number of knobs that allow variation of different model parameters. It generates event weights based on these variations that can be applied to the $\nu_{e}$ selected signal and background events. The effect of these uncertainties is computed in the same manner as the beam systematics. 
The complete list of parameters whose effect was studied can be found in [43]. The total uncertainty from this source on $\nu_{e}$ signal is $7.28 \%$ and on background is $2.14 \%$. The error on signal is dominated by the uncertainty on the axial mass $M_{A}$ in the quasi-elastic $\mathrm{CC}$ interactions for reasons touched upon earlier.

\subsection{Birks Suppresion}

It was noted in chapter 6 that tuning the scintillator quenching effect using the near detector data gives us a Birks Suppression constant value of $k_{B}=0.04 \mathrm{~g} / \mathrm{MeV} \mathrm{cm}^{2}$. This is 4 times higher than the typical values published in literature [44], although the negative contribution due to the second order Chou suppression mitigates over suppression to some extent. There is, however, some concern that with our measurement of Birks suppression, we are compensating for other unknown detector effects that result in loss of visible energy. We have taken the uncertainty on Birks suppression constants as a systematic to account for our ignorance of the exact parameterization of this effect.

We produced far and near simulation samples with Birks suppression parameters found in literature $\left(k_{B}=0.01 \mathrm{~g} / \mathrm{MeV} \mathrm{cm}{ }^{2}\right.$ and $\left.k_{C}=0.0 \mathrm{~g}^{2} / \mathrm{MeV}^{2} \mathrm{~cm}^{4}\right)$ and compared the extrapolation predictions from these samples with those from nominal samples.

\subsection{Decomposition Uncertainty}

In this analysis, we have opted to rely on the simulation to correctly model the ratio of different components in the $\nu_{e}$ selected background in the near detector. The excess in data is then proportionally distributed among the three beam background components. However, there is some indication that this isn't the case. In section 11.1, two alternative methods of decomposition were discussed. In one method, the $\mathrm{NC}, \nu_{\mu} \mathrm{CC}$ and beam $\nu_{e}$ CC scales were determined from energy distribution of events in the LID sideband. In the second method, the quasi-elastic, resonance and deep-inelastic interaction scales 
were determined from fitting the hadronic energy distribution of preselected events. Scaling interaction modes changes the relative rates of $\mathrm{NC}, \nu_{\mu} \mathrm{CC}$ and beam $\nu_{e} \mathrm{CC}$ interactions and this was introduced as a second method of decomposition. Table 11.1 shows the results in ND and table 11.2 shows the extrapolated background results in FD based on these decompositions. The error on decomposition is taken to be the largest deviation from the proportional decomposition, which is $7.22 \%$ based on table 12.2 .

\begin{tabular}{l|llll}
\hline Method & Tot bg. (\%) & $\mathrm{NC}(\%)$ & $\nu_{\mu} \mathrm{CC}(\%)$ & Beam $\nu_{e} \mathrm{CC}(\%)$ \\
\hline Scaled by type & 7.22 & 13.89 & -60.00 & 8.51 \\
Scaled by mode & 2.06 & -22.22 & 20.00 & 27.66 \\
\hline
\end{tabular}

Table 12.2: Percentage difference in predicted background with respect to the nominal proportional decomposition.

\subsection{Uncertainty on MRE Correction}

The MRE correction to the $\nu_{e}$ selection efficiency leads to a $15.6 \%$ increase in the expected appearance signal. The possible sources of error on this correction are evaluated here.

\section{$\nu_{\mu}$ Selection}

The MRE events are reconstructed from $\nu_{\mu} \mathrm{CC}$ interactions and the selection efficiency of $\nu_{\mu}$ 's is a smoothly rising function of the true neutrino energy. To evaluate the impact of changing the $\nu_{\mu}$ selection efficiency on the $\nu_{e}$ selection of MRE events, the cut on ReMID, the $\nu_{\mu}$ PID, was changed from the nominal and the new MRE efficiency corrections applied to the extrapolated appearance signal. It is found that this has very little effect on the MRE selection efficiency in the ND. Changing the $\nu_{\mu}$ selection cut largely only changes the background region of LID for MRE events as is seen in figure 12.3. Nevertheless, the MRE efficiency 
corrections after these changes were applied to the far detector appearance prediction and the size of the effect is taken as a systematic. Table 12.3 summarizes this.

\begin{tabular}{l|cc}
\hline$\nu_{\mu}$ Cut & Extrapolate $\nu_{e}$ Signal & Difference \\
\hline Nominal, ReMID >0.75 & 5.1 & 0 \\
Low, ReMID >0.55 & 5.07 & $0.59 \%$ \\
High, ReMID > 0.95 & 5.17 & $1.37 \%$ \\
\hline
\end{tabular}

Table 12.3: Change in MRE corrected extrapolated $\nu_{e}$ signal production due to different selection cuts on the MRE parent $\nu_{\mu} \mathrm{CC}$ interaction. The last column is the percentage difference with respect to the nominal cut of ReMID $>0.75$. 


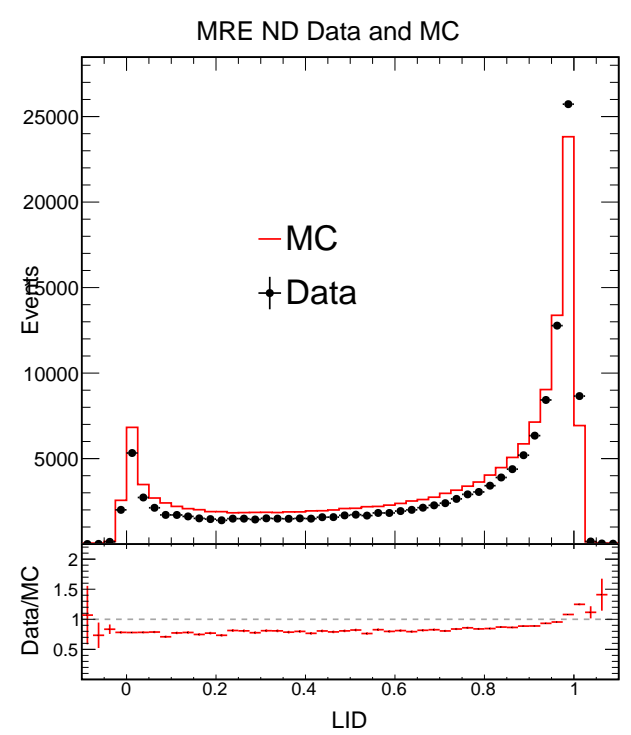

(a) LID distribution of MRE events, with ReMID $>0.55$ cut applied to parent interaction

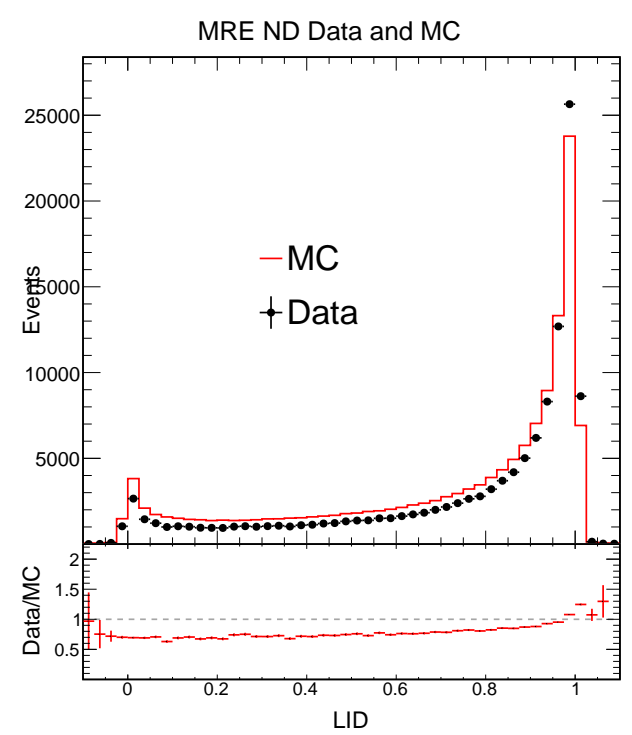

(b) LID distribution of MRE events, with ReMID $>0.95$ cut applied to parent interaction

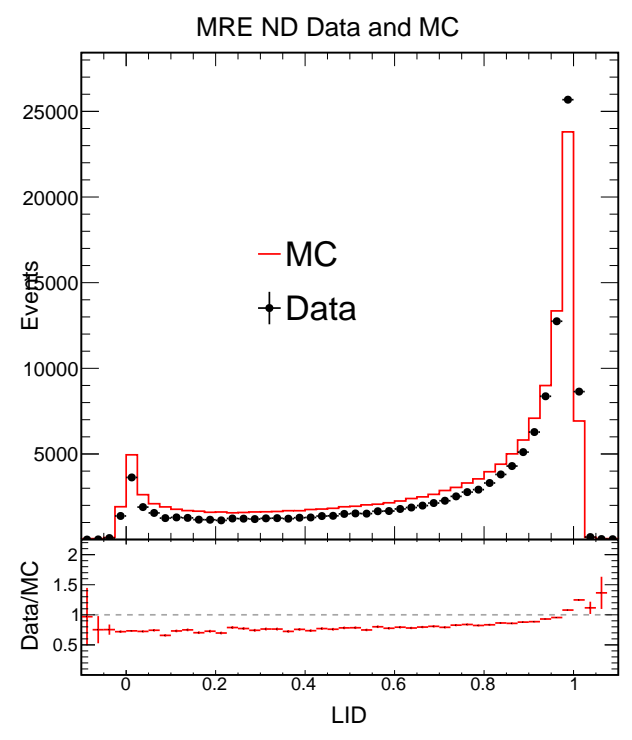

(c) LID distribution of MRE events, with the nominal ReMID $>0.75$ cut applied to parent interaction

Figure 12.3: Change in $\nu_{e}$ selection of MRff events upon changing the $\nu_{\mu}$ selection of the parent interactions. 


\section{MRE to Parent Slice Matching Efficiency}

It was noted in chapter 10 that the MRE to parent slice matching efficiency is discrepant between data and MC. To check if this has any bearing on the MRE correction, the efficiency cut was changed. Table 12.4 is a summary of these and their impact on the extrapolated signal prediction.

\begin{tabular}{l|cc}
\hline Efficiency Cut & Extrapolate $\nu_{e}$ Signal & Difference \\
\hline Nominal, Eff $>0.8$ & 5.10 & 0 \\
Eff $>0.00$ & 5.16 & $1.18 \%$ \\
Eff $>0.50$ & 5.16 & $1.18 \%$ \\
Eff $>0.95$ & 4.97 & $2.55 \%$ \\
\hline
\end{tabular}

Table 12.4: Change in MRE corrected extrapolated $\nu_{e}$ signal production due to different parent matching efficiency cuts on the MRE slice. The last column is the percentage difference with respect to the nominal cut of efficiency $>0.8$.

The maximum of the errors on the MRE correction are added in quadrature and the total error amounts to $2.89 \%$.

\subsection{Other Smaller Effects}

\subsubsection{Containment and Rock Contamination}

The NuMI beam is a line source of neutrinos for the near detector. The off-axis location of the detector leads to a non-uniform flux of neutrinos along the detector face. Moreover, the detector, being underground, is surrounded by rock where most beam neutrinos interact. Neutrino interactions in the rock preceding the front face of the detector often lead to activity in the front part of the detector. To study all these effects, the near detector was divided into octants, with each axes divided in half and the near detector data was extrapolated independently from each quadrant. The maximum 
variation produced in the background prediction from this exercise is of $2.7 \%$.

To reduce the time required in simulation of rock interactions that leave activity in the detector, a simplification in the simulation process is used. A large set of rock interactions is generated and overlaid with interaction of neutrinos in the detector. The reuse rate of a simulated rock interaction in the near detector simulation set used in this analysis is close to 350 times. If a single interaction passes selection cuts, it is likely to pass them for all the repetitions. This has been incorporated as a systematic, the size of which was determined by extrapolating without any rock interactions at all. The change in total background is $0.1 \%$.

\subsubsection{Simulation Statistics and Normalization}

The predictions for the analysis are made from limited simulation statistics. The error on the prediction due to this is $3.47 \%$ on background and $0.9 \%$ on signal.

Normalization errors arise from two source - POT counting and detector mass. The POT counting error is $0.5 \%$ [45]. The detector mass uncertainty is computed from tolerances on the size, mass and densities of the detector components and adds up to $0.7 \%$ of the detector mass [46]. Also, a difference of $0.8 \%$ in the neutrino event rate is observed in the near detector data and MC before any cuts are applied. It is not clear whether this applies equally to the far detector as well. Therefore, it is treated as a normalization systematic. Additionally, the diblock configurations in real conditions far detector simulation are not identical to the data; this contributes another $0.7 \%$ to the normalization systematic. All these uncertainties add up to $1.37 \%$.

\subsubsection{Alignment}

The analysis simulation samples use a perfectly aligned geometry of the near and far detectors. The installation of the blocks is indeed not perfect and they are staggered with respect to one another. Small samples of near and far detector simulations were generated with this imperfect alignment and it was shown to have a small effect on 
reconstruction and selection efficiencies. The error due to this is $0.73 \%$ on signal and $1.73 \%$ on background.

\subsubsection{Light Level}

It was discussed earlier that the far detector calibrated response shows different shapes between data and MC as a function of distance from readout. The suspicion is that the scintillator light yield is not properly tuned in the simulation. The impact of this effect on the energy reconstruction is accounted for in the calibration systematic. However, a mistuned light level can lead to second order effects, such as hits below threshold, that never triggered. The number of hits in a slice shifts by about $5 \%$ in the response slope simulated sample. This $5 \%$ shift results in a $1 \%$ change in the signal selection efficiency, while there is no noticeable effect on background efficiency.

\subsection{Summary}

Many different sources of error have been considered for this analysis. The largest of errors on signal arise from uncertainty in calibration, neutrino interaction model and Birks suppression parameters. The errors are tabulated in table 12.5.
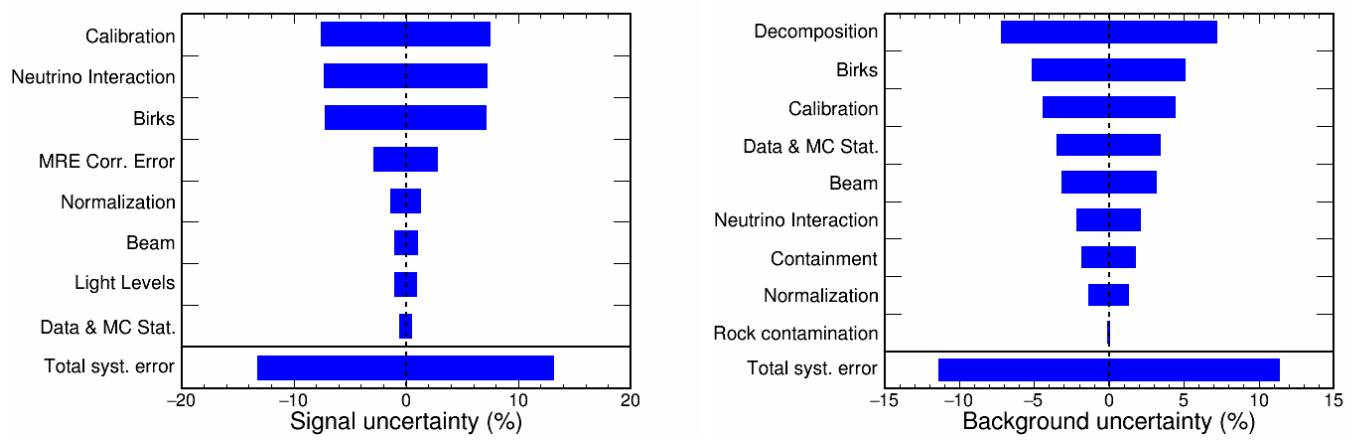

Figure 12.4: Summary of signal and background uncertainties 


\begin{tabular}{|l|c|c|}
\hline & Total BG Syst. (\%) & Signal Syst. (\%) \\
\hline Beam & 3.18 & 1.06 \\
Birks & 5.14 & 7.22 \\
Calibration & 4.44 & 7.58 \\
Light Level & $\mathrm{n} / \mathrm{a}$ & 1.00 \\
Neutrino Interaction & 2.14 & 7.28 \\
Containment & 1.84 & $\mathrm{n} / \mathrm{a}$ \\
Rock contamination & 0.10 & $\mathrm{n} / \mathrm{a}$ \\
Decomposition & 7.22 & $\mathrm{n} / \mathrm{a}$ \\
ND Data \& MC Stats & 3.47 & 0.6 \\
Normalization & 1.37 & 1.37 \\
MRE Correction & $\mathrm{n} / \mathrm{a}$ & 2.89 \\
\hline Total & 11.41 & 13.24 \\
\hline
\end{tabular}

Table 12.5: Final systematic error (in percentages) on the combined background and signal in the Far Detector. The last row is the sum in quadrature of all errors in this table. 


\section{Chapter 13}

\section{Results of $\nu_{e}$ Appearance Analysis}

The last two chapters discussed in detail the predicted signal and background in the $\nu_{e}$ appearance analysis of $\mathrm{NO} \nu \mathrm{A}$ 's first data and the errors on these predictions. The predictions are reviewed again in table 13.1.

\begin{tabular}{l|ccc|ccc|c}
\hline & Signal & Stat & Syst & Total Bg & Stat & Syst & Total Events \\
\hline Simple Osc & 5.10 & 2.26 & 0.68 & 0.97 & 0.98 & 0.11 & 6.07 \\
$\mathrm{IH}, \delta=\pi / 2, \theta_{23}=\pi / 4$ & 2.59 & 1.61 & 0.34 & 0.96 & 0.98 & 0.11 & 3.55 \\
$\mathrm{NH}, \delta=3 \pi / 2, \theta_{23}=\pi / 4$ & 6.57 & 2.56 & 0.87 & 0.94 & 0.97 & 0.11 & 7.54 \\
\hline
\end{tabular}

Table 13.1: Expected signal and background for $\mathrm{NO} \nu \mathrm{A}$ first data along with the systematic and statistical errors on the predictions.

This chapter presents the results of $\nu_{e}$ appearance analysis performed on the first data collected with the $\mathrm{NO} \nu \mathrm{A}$ detectors. The first section presents a sideband study that was done prior to unblinding the main signal region, as a final check of the analysis chain. The second section presents the main result and its statistical treatment. The final section is a discussion of the outlook for future analyses of $\mathrm{NO} \nu \mathrm{A}$ data. 


\subsection{PID Sideband}

The signal events for this analysis are those that pass all $\nu_{e}$ preselection cuts and have a LID value greater than 0.95 . To test the analysis chain and check that the PID behaves as expected on data, the LID sideband from 0.7 to 0.95 was unblinded ahead of the final analysis. The event counts expected from extrapolation for this region in the FD beam data are listed in table 13.2. The MRE correction has been applied to the signal prediction here. The correction was determined separately using MRE events with LID values between 0.7 and 0.95 .

\begin{tabular}{l|ccc|c}
\hline Osc,. Params & Signal & Beam BG & Cosmic BG & Tot. Events \\
\hline Simple osc. & 2.33 & 1.50 & 0.38 & 4.21 \\
IH,$\delta=\pi / 2$ & 1.22 & 1.51 & 0.38 & 3.11 \\
NH, $\delta=3 \pi / 2$ & 3.01 & 1.48 & 0.38 & 4.87 \\
\hline
\end{tabular}

Table 13.2: LID sideband event counts expectation in the far detector

In the FD data, 5 events have been observed in the sideband, which is consistent with the expectations listed in table 13.2. All the events observed appear neutrino-like and the PID performance seems well-behaved on the FD data.

\section{2 $\nu_{e}$ Appearance Result}

In the far detector NuMI beam data, 6 events have been observed in the signal region. The event displays of these 6 candidates follow in figures 13.3, 13.4 and 13.5. All the observed candidates have very convincing electron neutrino characteristics, with a well-defined electromagnetic shower. Figure 13.1 shows the cumulative event count as a function of time during the data taking period along with the integrated exposure expressed in mass $\times$ POT. The Kolmogorov-Smirnov $(\mathrm{KS})$ statistic test of the integrated event count with the exposure as a reference has a probability of $31 \%$, indicating that 
the observed event rate is consistent with exposure.

Figure 13.2 shows the time distribution of the candidate events. The blue lines mark the edges of the NuMI timing windows and the two out-of-time events are the cosmic background. Interestingly, no $\nu_{e}$ candidates are found in the delayed $64 \mu$ s time window in the pre-shutdown data. Figure 13.6 shows distributions of event energy, cosine of angle wrt to NuMI beam direction and electron log-likelihoods of the signal candidates overlaid on the prediction from simulation. These distributions show no unexpected behavior in the candidates observed in the FD data. Figure 13.7 shows the vertex distributions of the candidates. With the exception of one event, which is very close to the front face of the detector, all events are well contained within the fiducial volume of the detector.

Figures 13.8 and 13.9 are the longitudinal and transverse $d E / d x$ profiles of the candidate events overlaid on the $d E / d x$ probability distribution constructed from electrons in simulated $\nu_{e} \mathrm{CC}$ interactions. The events follow the trademark electron shower development, with energy deposition consistent with a MIP in the first few planes and with an electromagnetic shower in the following planes. 


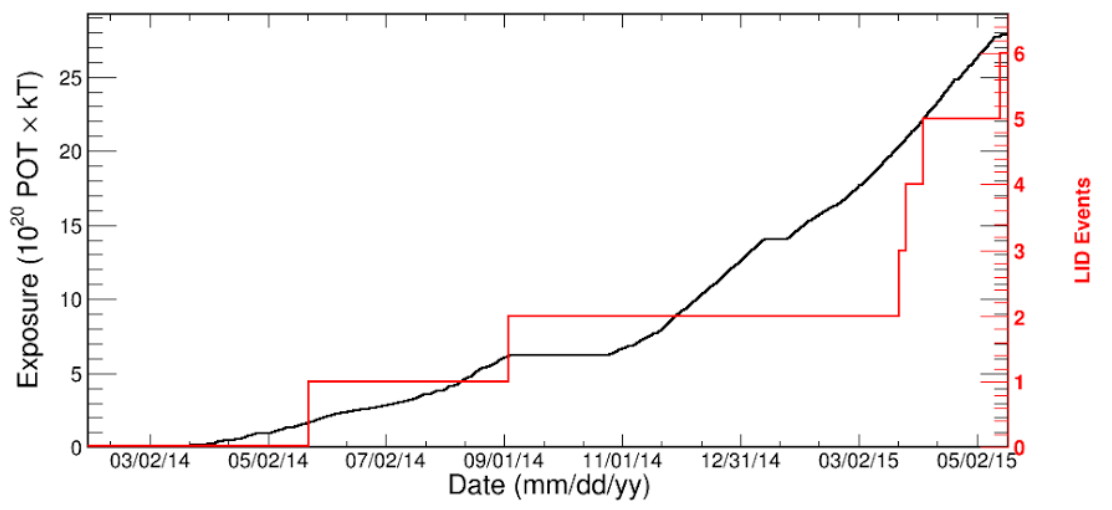

Figure 13.1: $\nu_{e}$ Appearance candidates in FD NuMI beam data. The gray events are are out-of-time cosmics.

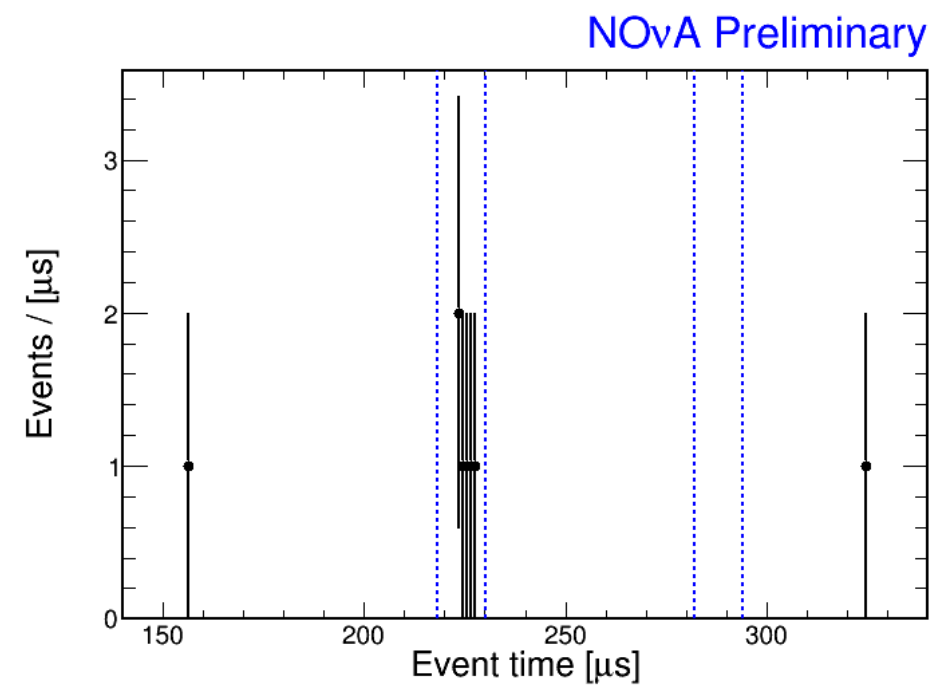

Figure 13.2: Timing distribution of FD signal events. The blue line indicate the NuMI beam window boundaries. The two out of time events are cosmic background. 


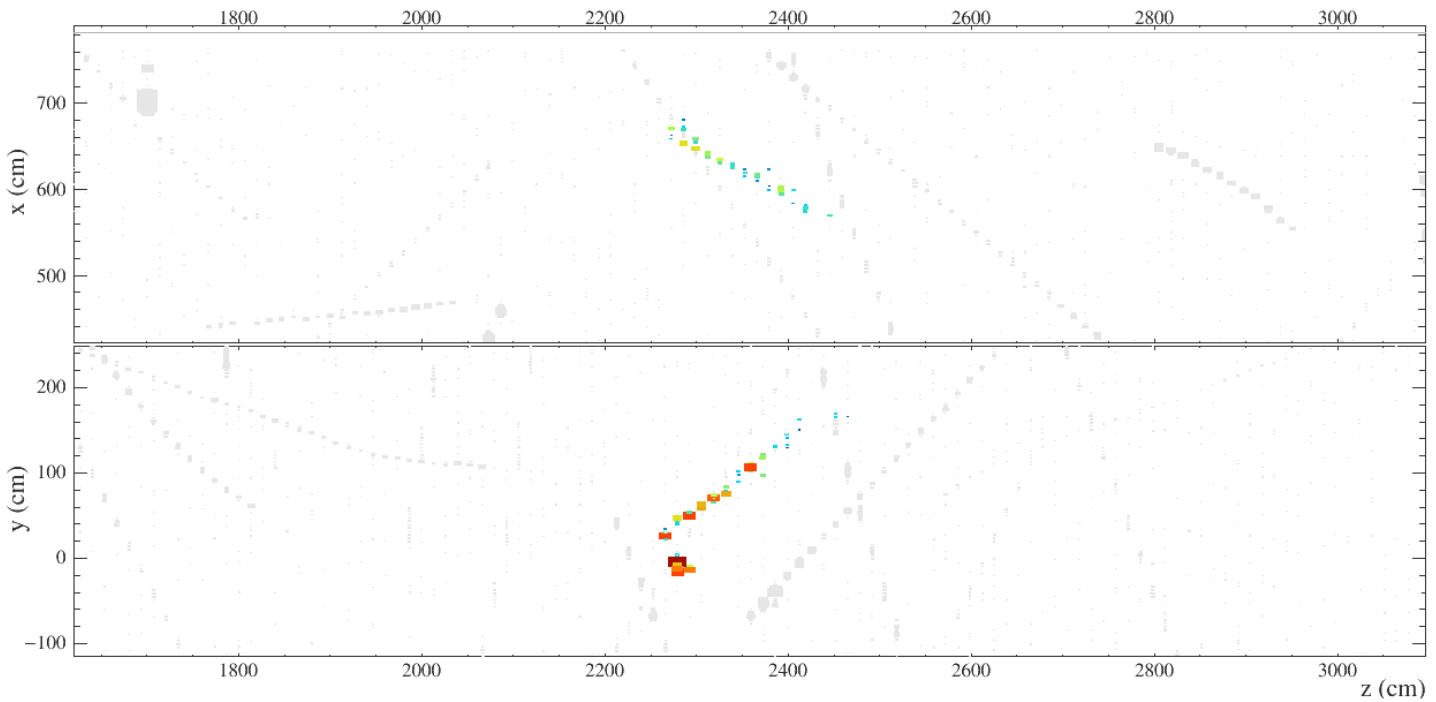

NOvA - FNAL E929

Run: $15330 / 4$

Event: $11978 /$.

UTC Fri May 23, 2014

17:30:2.632293184
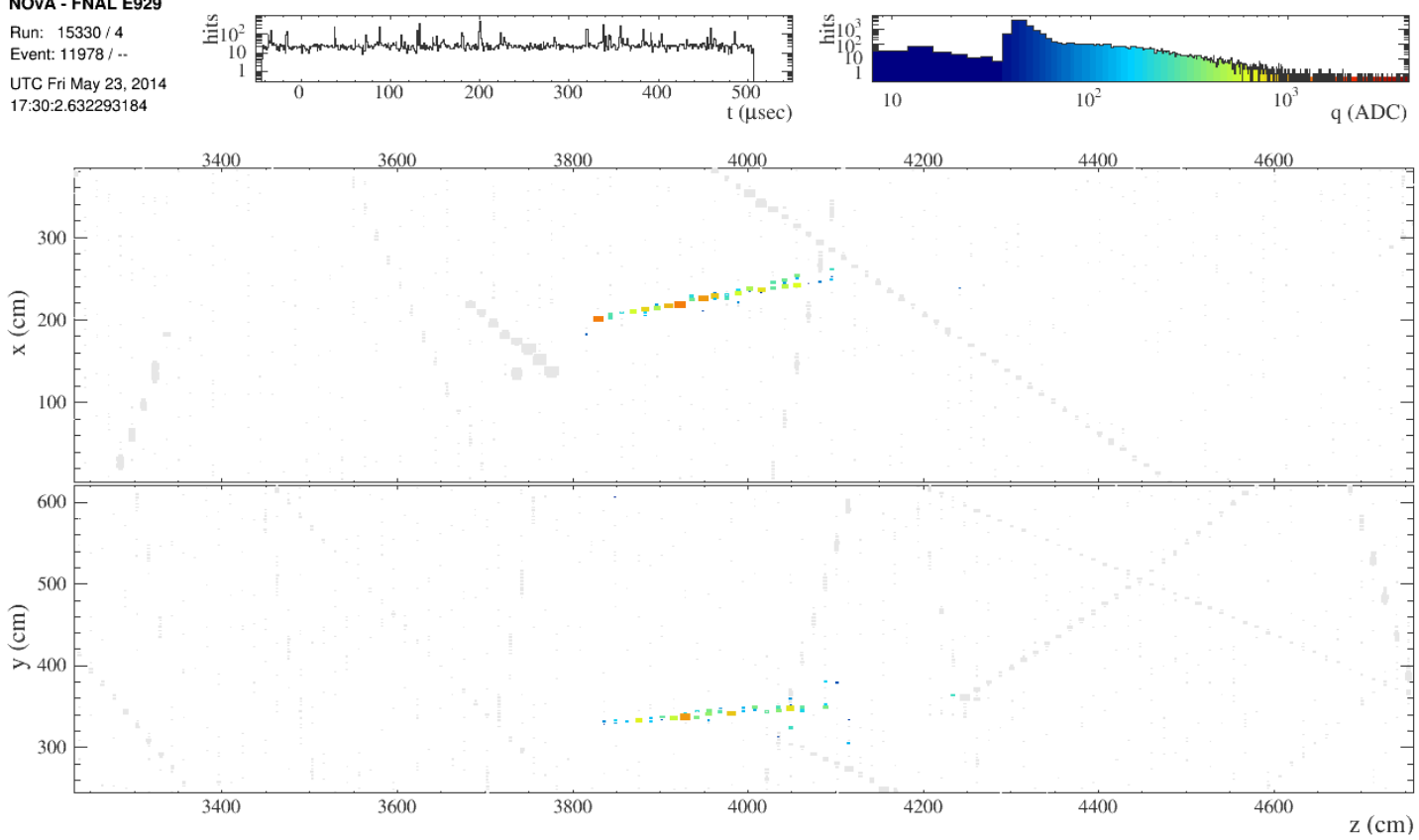

NOvA - FNAL E929

Run: $17103 / 7$

UTC Wed Sep 3, 2014
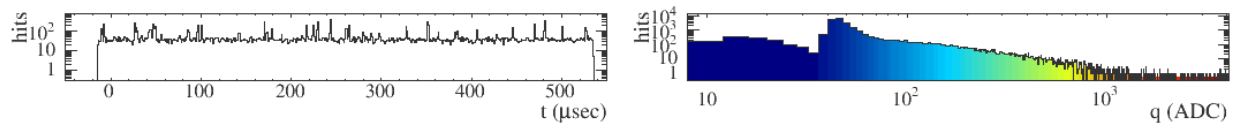

Figure 13.3: $\nu_{e}$ Appearance candidates in FD NuMI beam data. The gray events are are out-of-time cosmics. 

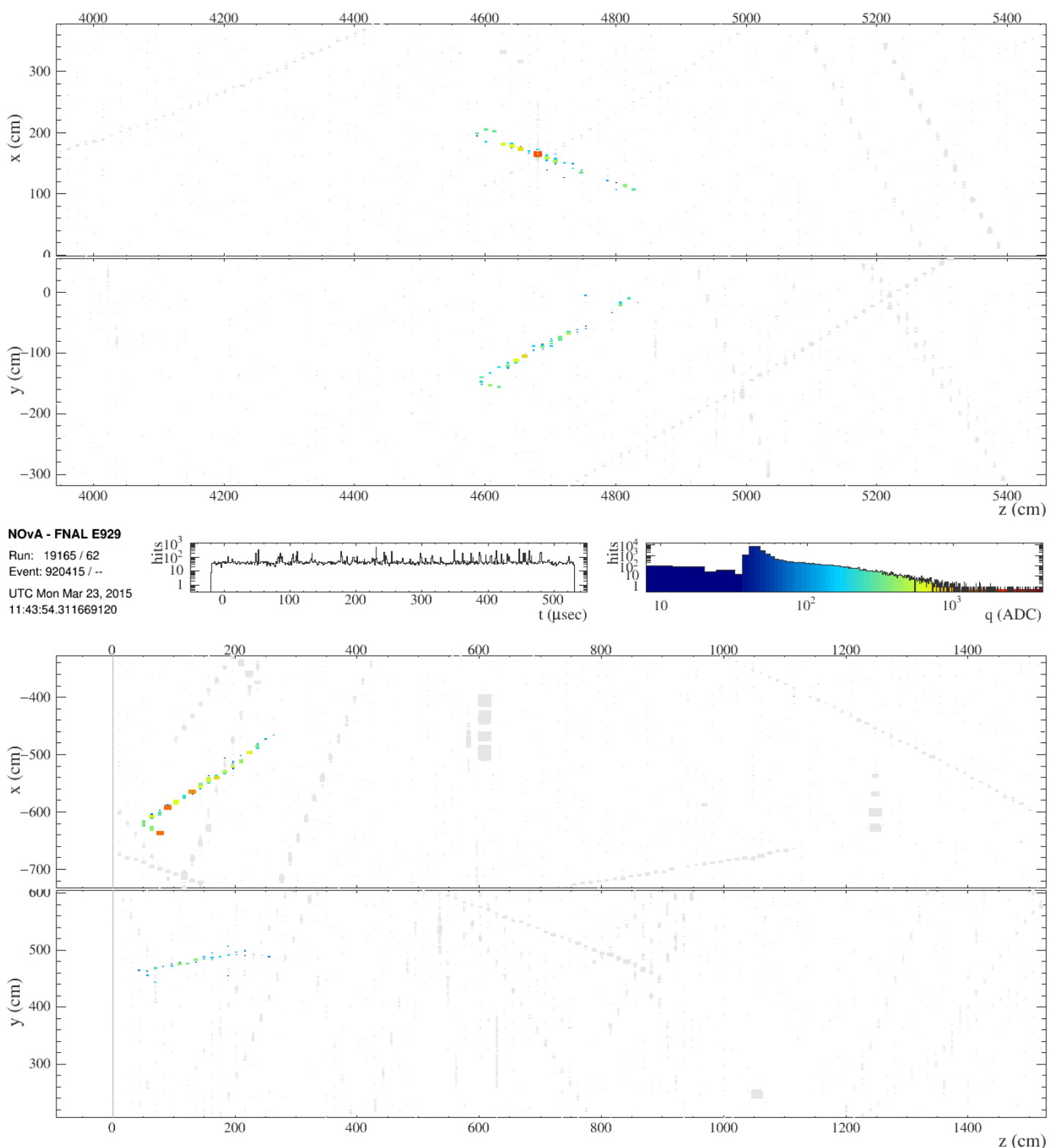

NOvA - FNAL E929 Run: $19193 / 13$

UTC Fri Mar 27, 2015
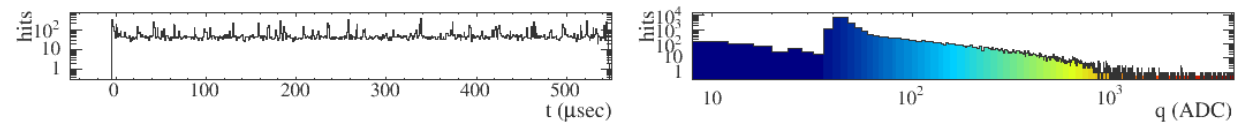

Figure 13.4: $\nu_{e}$ Appearance candidates in FD NuMI beam data. The gray events are are out-of-time cosmics. 

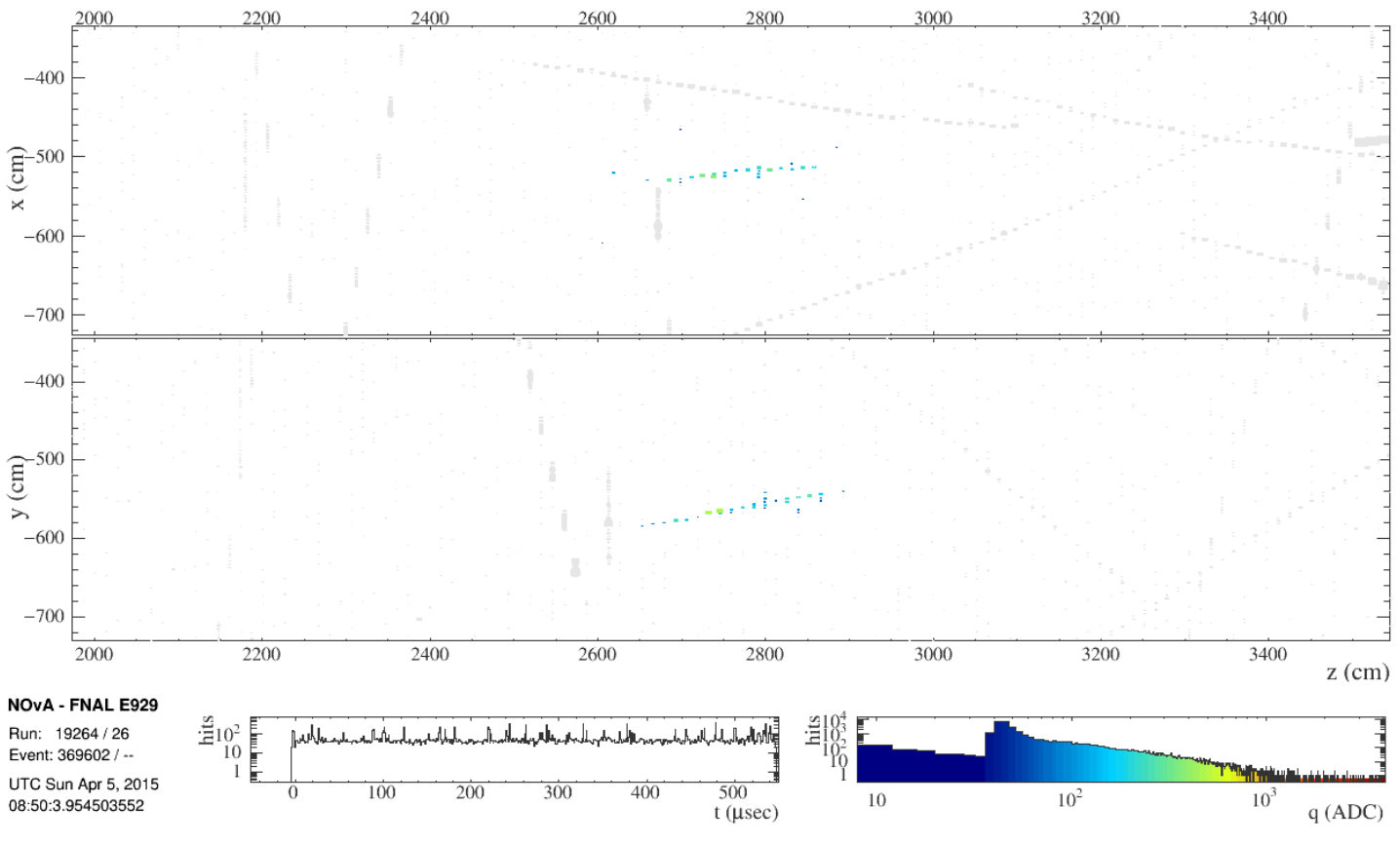
08:50:3.954503552 4000 4200 4400 4600 4800

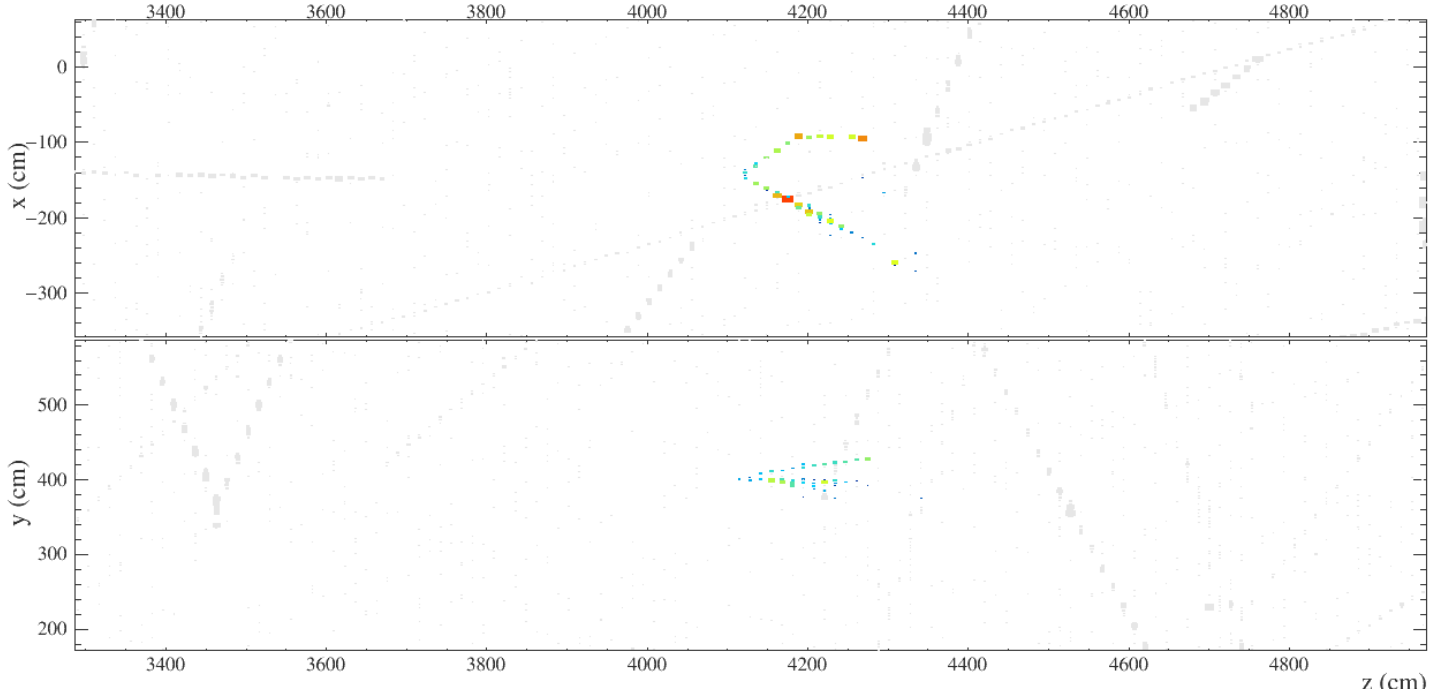

NOvA - FNAL E929 Run: 19578/5
Event: 98069/.. UTC Thu May 14, 2015 17:55:39.044985484
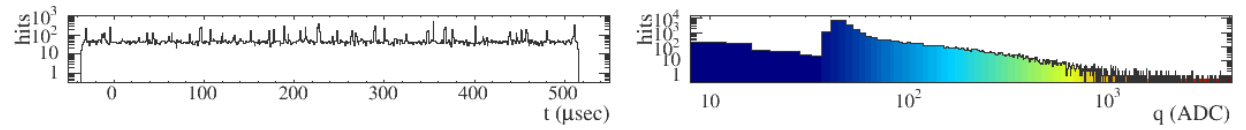

Figure 13.5: $\nu_{e}$ Appearance candidates in FD NuMI beam data. The gray events are are out-of-time cosmics. 

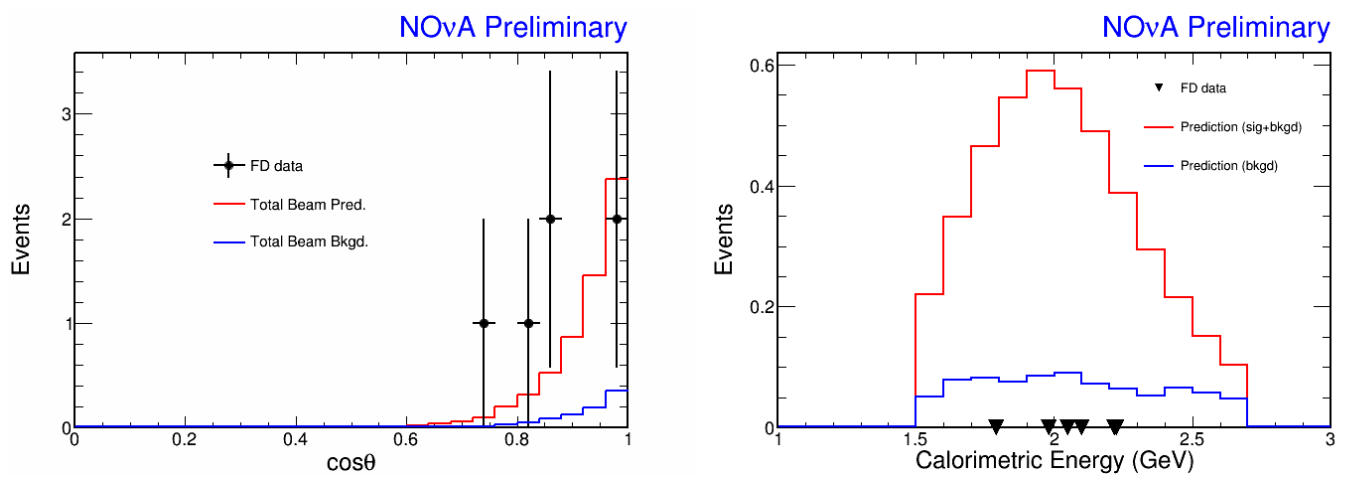

(a) Cosine of angle with respect to the beam (b) Energy distribution of neutrinos. Each ardirection. row indicates the observed candidate events in the FD data

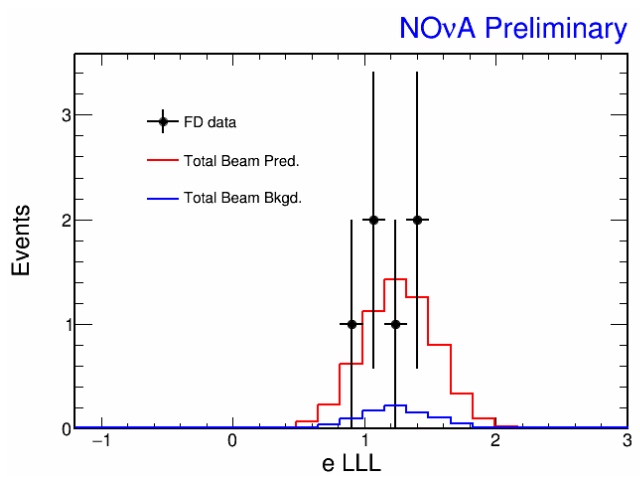

(c) Longitudinal electron log-likelihood

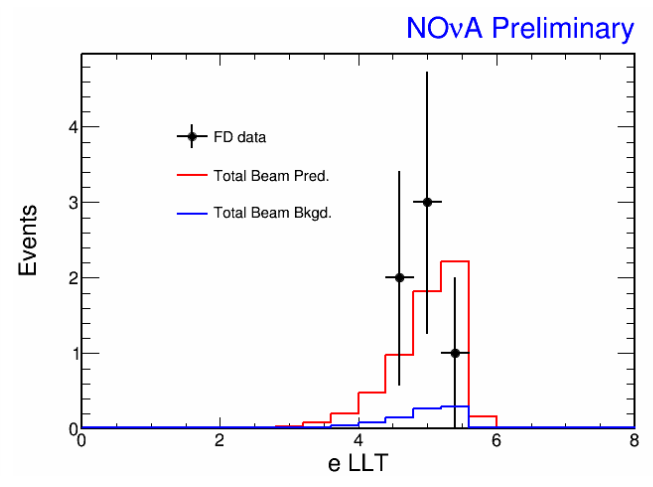

(d) Transverse electron log-likelihood

Figure 13.6: Distributions of signal events in the NuMI beam window in the FD data.

\subsection{Analysis of the Result}

In the analysis presented here, the only information used to infer neutrino oscillation parameters is the number of candidate events observed in the FD NuMI data. No energy shape information is utilized. This section discusses a simple method of analyzing the results in the first subsection, followed by a more sophisticated statistical treatment. 


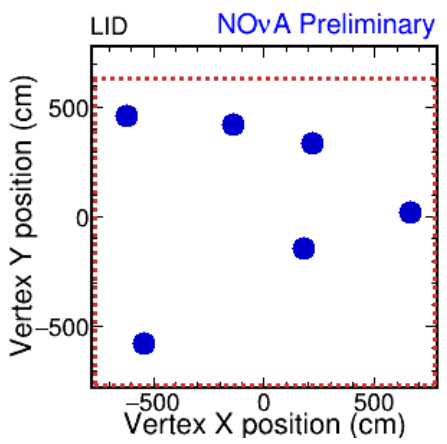

(a) Vertex distribution of candidate events in $\mathrm{XY}$ plane

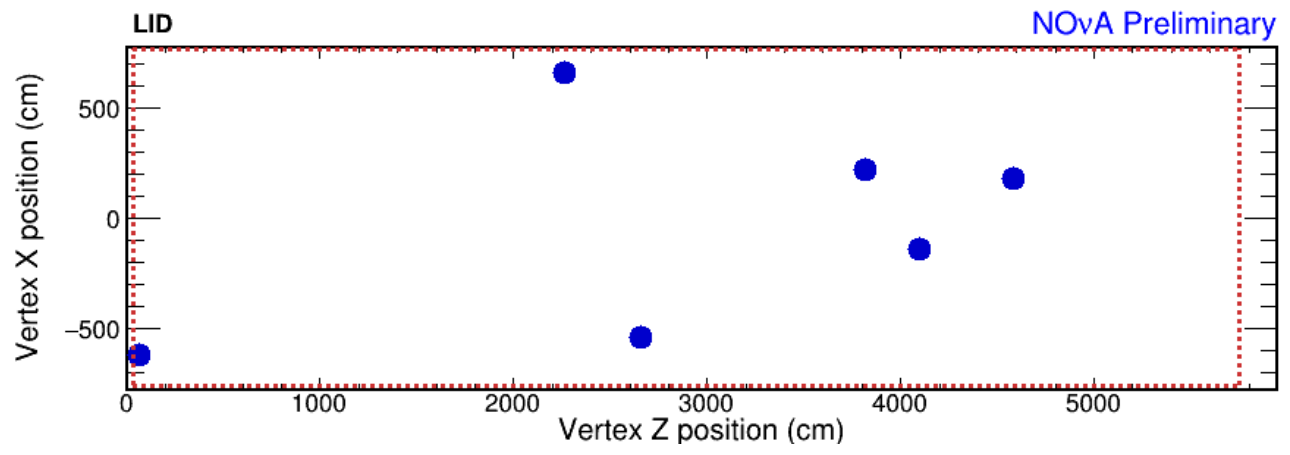

(b) Vertex distribution of candidate events in $\mathrm{XZ}$ plane

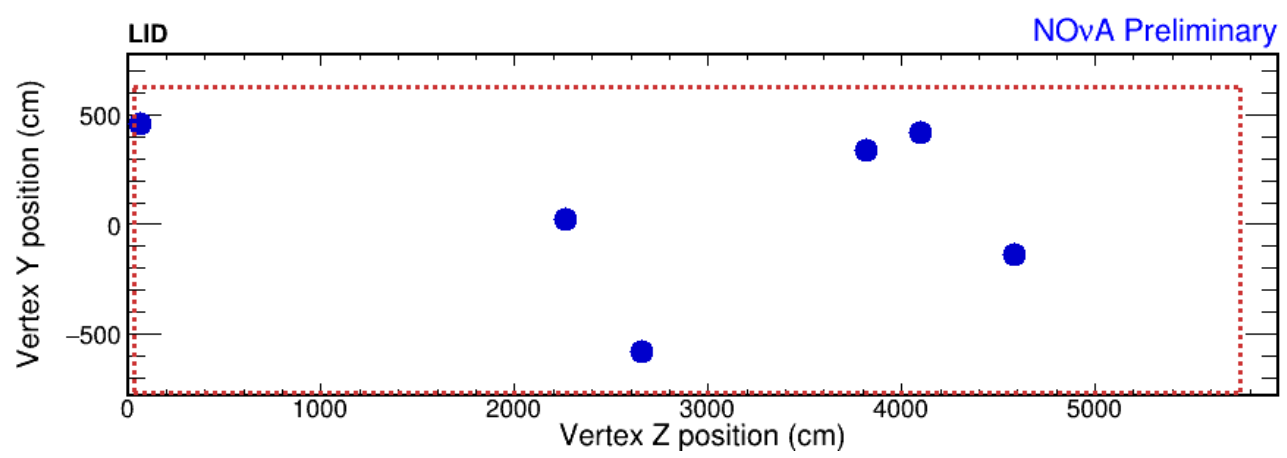

(c) Vertex distribution of candidate events in YZ plane

Figure 13.7: Vertex distributions of candidate events. The dotted red lines indicate the containment boundaries. 


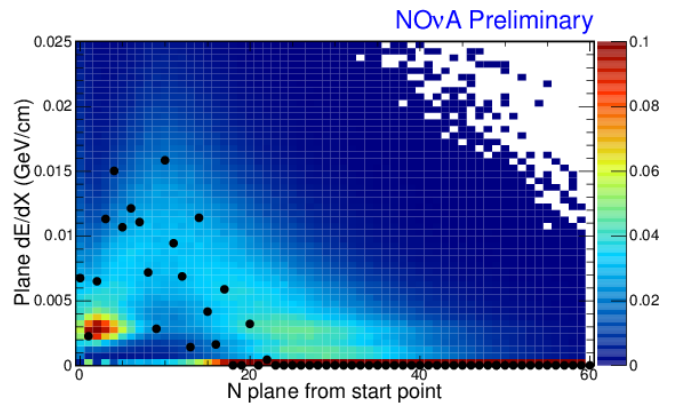

(a) $d E / d x$ in longitudinal direction vs plane number from shower start for $\nu_{e}$ candidate event in run 15330

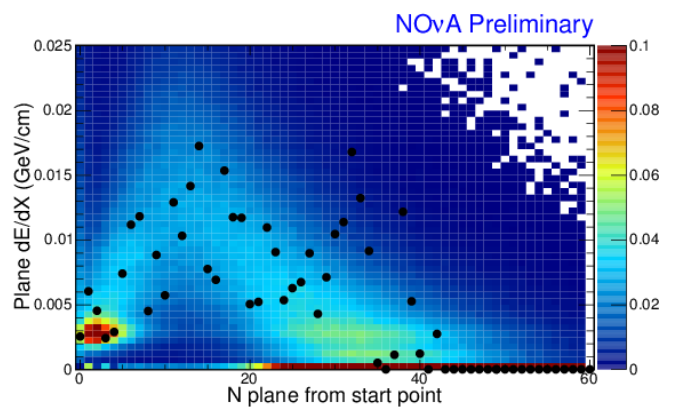

(c) $d E / d x$ in longitudinal direction vs plane number from shower start for $\nu_{e}$ candidate event in run 17103

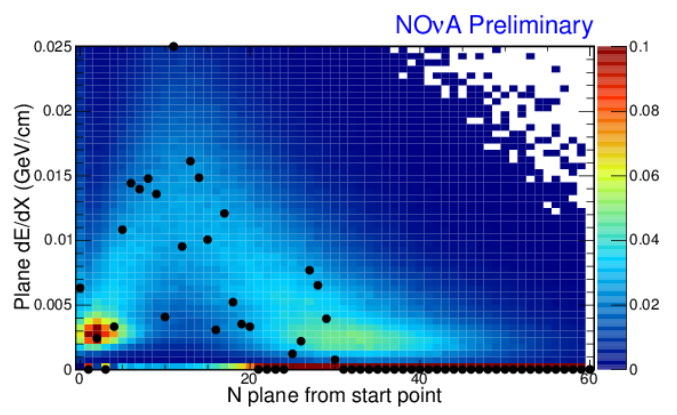

(e) $d E / d x$ in longitudinal direction vs plane number from shower start for $\nu_{e}$ candidate event in run 19165

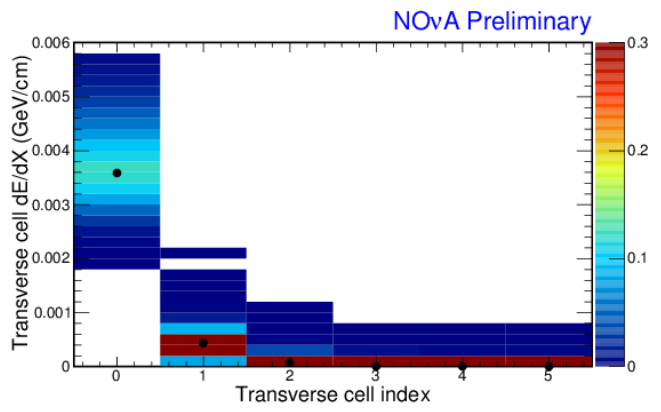

(b) $d E / d x$ in transverse direction vs cell number from shower core for $\nu_{e}$ candidate event in run 15330

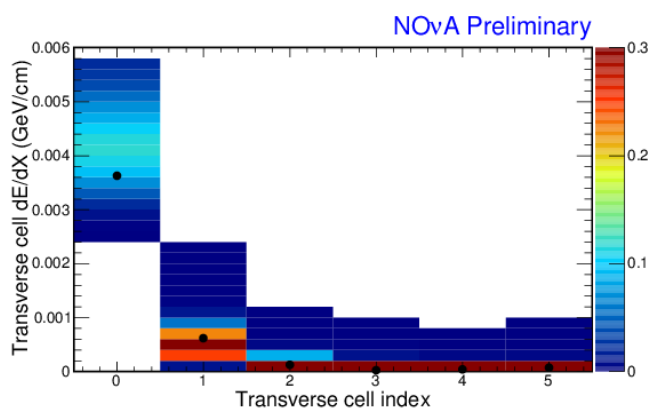

(d) $d E / d x$ in transverse direction vs cell number from shower core for $\nu_{e}$ candidate event in run 17103

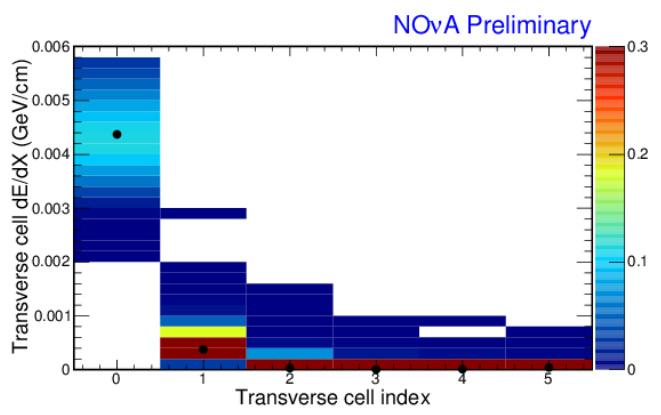

(f) $d E / d x$ in transverse direction vs cell number from shower core for $\nu_{e}$ candidate event in run 19165

Figure 13.8: $d E / d x$ by plane number from the shower start in the longitudinal and transverse directions for leading showers. The points are the data and the color scale is the $d E / d x$ probability distribution for electrons in simulated $\nu_{e} \mathrm{CC}$ events. 


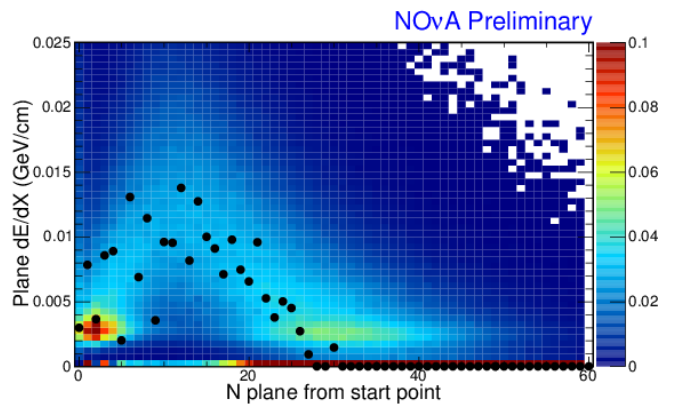

(a) $d E / d x$ in longitudinal direction vs plane number from shower start for $\nu_{e}$ candidate event in run 19193

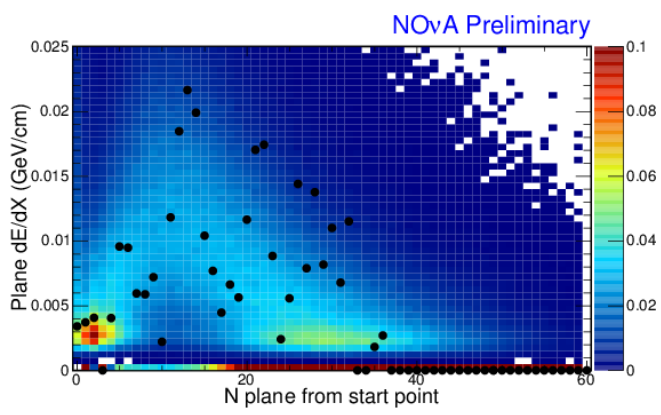

(c) $d E / d x$ in longitudinal direction vs plane number from shower start for $\nu_{e}$ candidate event in run 19264

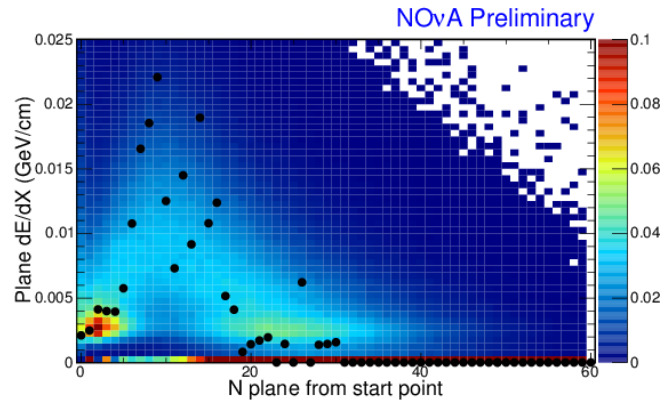

(e) $d E / d x$ in longitudinal direction vs plane number from shower start for $\nu_{e}$ candidate event in run 19578

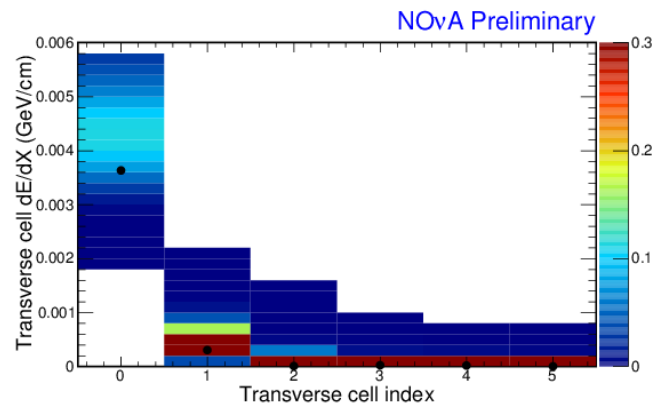

(b) $d E / d x$ in transverse direction vs cell number from shower core for $\nu_{e}$ candidate event in run 19193

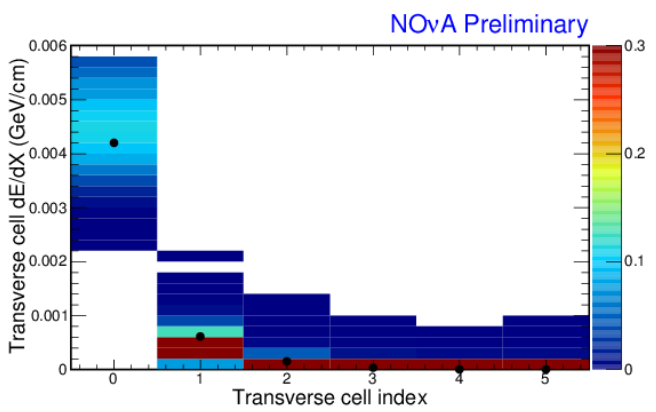

(d) $d E / d x$ in transverse direction vs cell number from shower core for $\nu_{e}$ candidate event in run 19264

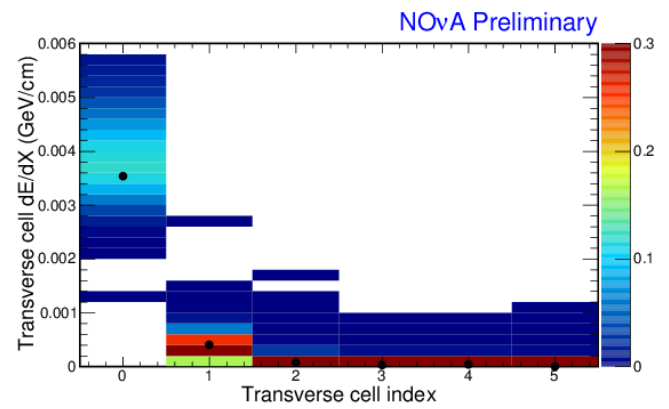

(f) $d E / d x$ in transverse direction vs cell number from shower core for $\nu_{e}$ candidate event in run 19578

Figure 13.9: $d E / d x$ by plane number from the shower start in the longitudinal and transverse directions for leading showers. The points are the data and the color scale is the $d E / d x$ probability distribution for electrons in simulated $\nu_{e} \mathrm{CC}$ events. 


\subsubsection{Significance of Observation: Simple Treatment}

In the far detector data, 6 events have been observed in the $\nu_{e}$ appearance channel. Recall that the estimated background is 0.97 event with a systematic error of $11.64 \%$. The probability that 6 or more events may be observed due to Poisson fluctuation of this background, rather than due to $\nu_{\mu}$ to $\nu_{e}$ oscillation is given by :

$$
p=\sum_{x=6}^{\infty} \int_{-\infty}^{-\infty} \operatorname{Poisson}(\mathrm{x}, \mathrm{b}) \times \operatorname{Gauss}\left(\mathrm{b}, \mathrm{b}_{0}, \sigma_{\mathrm{b}} \mathrm{b}_{0}\right) \times \frac{1}{\sigma_{\mathrm{b}} \mathrm{b}_{0}} \mathrm{db}
$$

Here, $b_{0}$ and $\sigma_{b}$ are the expected background and the relative systematic error on the background, respectively. The Gaussian varies the background with a width equal to the systematic error and the Poisson function fluctuates it around the new central value.

If simple oscillations are used, with no matter effects or CP violation, the probability of 6 events being an upward fluctuation of the background is $5.5 \times 10^{-4}$, which translates to a significance of rejection of no-oscillation hypothesis at $3.46 \sigma$.

To see which of the two extreme hierarchy and CP violation scenarios are favored by our data, we perform the same calculation as above (eq. 13.1) except that the background and background error are replaced by the expected total event count for the particular oscillation hypothesis and the total error. From this computation, the inverted hierarchy with CP violation phase $\delta=\pi / 2$ parameters are disfavored at 1.28 $\sigma$, while the normal hierarchy with $\delta=3 \pi / 2$ is disfavored at $0.73 \sigma$. Therefore, our data mildly favor the normal hierarchy with $\delta=3 \pi / 2$ at $1.05 \sigma$.

\begin{tabular}{l|c}
\hline Deviation from... & Significance in $\sigma$ \\
\hline No-oscillation hypothesis & 3.46 \\
$\mathrm{IH}, \delta=\pi / 2$ & 1.28 \\
$\mathrm{NH}, \delta=3 \pi / 2$ & 0.74 \\
\hline
\end{tabular}

Table 13.3: Significance of observation 


\subsubsection{Fit to Oscillation Parameters}

The oscillation fits here are performed in the $2 \mathrm{D}$ space of $\sin ^{2} 2 \theta_{13}$ versus CP violation phase $\delta$. Mass hierarchy can also produce an enhancement or suppression of oscillations, but since it can only take on of two values $\left(\Delta m_{32}^{2}>0\right.$ for normal hierarchy and $\Delta m_{32}^{2}<0$ for inverted hierarchy), the fit values are computed and plotted separately for the two hierarchy hypotheses. The systematic uncertainty on the signal and the background are treated as additional uncertainty along with the statistical error. Since statistical uncertainty in this analysis is fairly large, treating systematics as nuisance parameters is found to make little difference.

In the fit for $\sin ^{2} 2 \theta_{13}$, a marginalization is performed over the atmospheric and solar oscillation parameters. The atmospheric parameter measurements are taken from the $\mathrm{T} 2 \mathrm{~K}$ experiment result in [47] because the dependence of their result on hierarchy is small due to a much shorter baseline. The solar oscillation parameters are from [6]. The results of this fit are in figure 13.10 .

Figure 13.10 shows that the $\nu_{e}$ appearance result from $\mathrm{NO} \nu \mathrm{A}$ data is well in agreement with the value of $\sin ^{2} 2 \theta_{13}$ measured by the reactor neutrino experiments. The best fit point in the normal hierarchy case is at $\sin ^{2} 2 \theta_{13}=0.092$ and $\delta / \pi=0.31$. For inverted hierarchy, the best fit is at $\sin ^{2} 2 \theta_{13}=0.10$ and $\delta / \pi=1.64$. There is a slight tension with the reactor results for $\sin ^{2} 2 \theta_{13}(1-2 \sigma)$ in case of inverted hierarchy, CP violation phase $\delta \sim \pi / 2$, where larger values of $\sin ^{2} 2 \theta_{13}$ are favored by our observation of 6 events. For the normal hierarchy, the result is consistent with the reactor constraint for all values of $\delta$ within $1 \sigma$.

Since the $\sin ^{2} 2 \theta_{13}$ parameter is precisely measured in reactor neutrino experiments, it can be used as a constraint to check which values of $\mathrm{CP}$ violating phase our data are most consistent with. This is shown in figure 13.11a where the likelihood difference with respect to best fit is plotted as a function of $\delta$. This also points towards a greater consistency with the normal hierarchy compared to inverted. In IH case, a $\delta$ value 


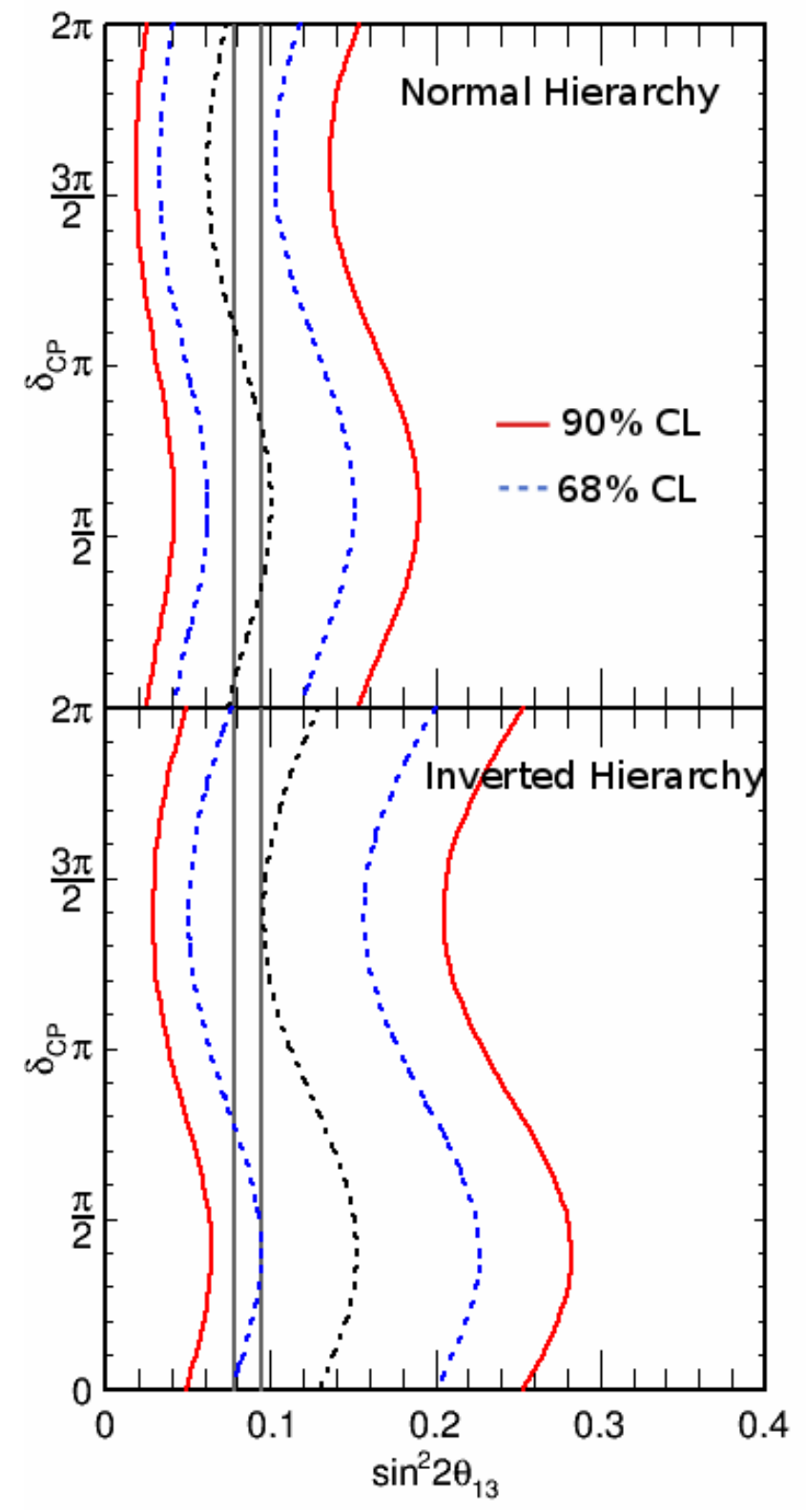

Figure 13.10: A fit to $\theta_{13}$ and $\mathrm{CP}$ violation phase, $\delta$, based on the observation of 6 events in the $\nu_{e}$ appearance channel, where the expected background is 0.97 event. The dotted blue lines enclose the $68 \%$ confidence interval and the solid red lines are $90 \%$ confidence interval. The solid gray lines show the $\sin ^{2} 2 \theta_{13}$ measurement from the reactor experiments. 
close to $3 \pi / 2$ is preferred and in NH case, values close to 0 and $\pi$ are favored. This is because the observed event count of 6 falls short of the predicted event count for $\delta=3 \pi / 2$ in normal hierarchy case, and is too high for the other extreme of inverted hierarchy and $\delta=\pi / 2$. This puts our measurement of 6 events close to the confused region on the biprobability space (figure 13.11b) where the two ellipses due to inverted and normal hierarchies begin to overlap. However, more data need to be collected to make a definitive statement on these parameters.
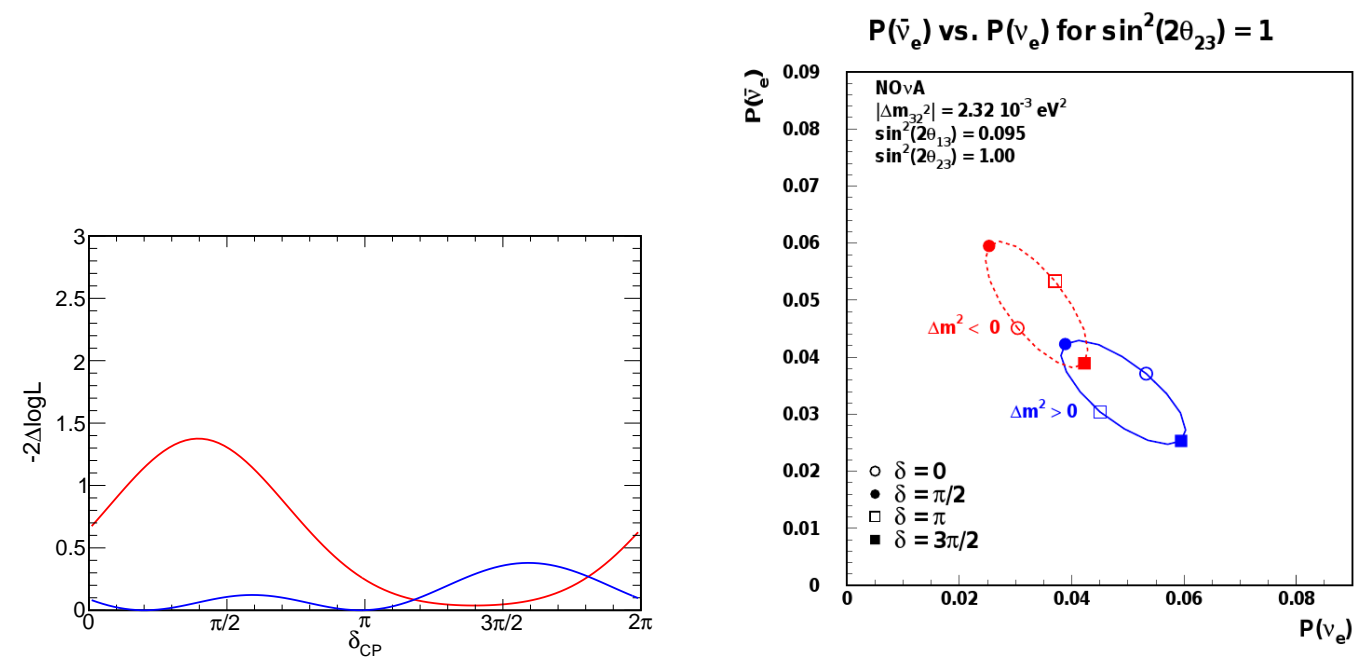

(a) $-2 \Delta \log L$ as a function of $\delta$ with $\sin ^{2} 2 \theta_{13}$ (b) For reference, the behavior of $\nu_{e}$ and $\bar{\nu}_{e}$ apfixed at the reactor value of 0.086 pearance for normal and inverted hierarchies for different values of $\delta$

Figure 13.11: $-2 \Delta \log L$ as a function of $\delta$ with $\sin ^{2} 2 \theta_{13}$ fixed at the reactor value of 0.086. Comparison with biprobability plot on the right indicates that the current observation is somewhere between the two extreme oscillation scenarios $(\mathrm{NH}, \delta=3 \pi / 2$ and $\mathrm{IH}, \delta=\pi / 2)$ 


\subsubsection{Feldman-Cousins Method for Confidence Interval}

The oscillation parameters like $\sin ^{2} \theta_{13}$ have a well-defined physical range, in this case 0 to 1 . However, the Gaussian errors on such parameters can sometimes extend beyond the physical range, where they have no natural interpretation. If these cases are not carefully dealt with, the quoted confidence intervals can often lead to under- or overcoverage. The complete presentation of the subtleties involved in the analysis of such parameters can be found in the original Feldman-Cousins (FC) publication [48]; what follows is a brief description of their proposed method to circumvent these problems.

In an ensemble of experiments with an observable $x$ that depends on a parameter $\mu$, a confidence interval $\left[\mu_{1}, \mu_{2}\right]$ on the parameter $\mu$ is defined as a subset that satisfies

$$
P\left(x \mid \mu \in\left[\mu_{1}, \mu_{2}\right]\right)=\alpha
$$

Therefore, the interval contains the true value $\mu_{t}$ of the parameter $\mu$ in $\alpha$ fraction of the experiments. To determine the confidence interval, the outcomes are added in the decreasing order of probability, starting with $P\left(x \mid \mu_{\mathrm{mp}}\right)$, where $\mu_{\mathrm{mp}}$ maximizes the probability of the observed outcome, until the summed probability reaches the desired level $\alpha$. In the FC method, the best fit value $\mu_{\mathrm{mp}}$ is constrained to be within its physical range. Moreover, the order in which probabilities are added, to construct a confidence interval is instead determined by the likelihood ratio $P(x \mid \mu) / P\left(x \mid \mu_{\mathrm{mp}}\right)$. Since in the Gaussian regime, $-2 \ln P \sim \chi^{2}$, the likelihood ratio can be replaced by the difference between the $\chi^{2}$ at a given value of $\mu$ and that at $\mu_{\mathrm{mp}}$, which we call $\chi_{\mathrm{mp}}^{2}$.

Prior to looking at the data, for each point in the oscillation phase space, an ensemble of statistical experiments is generated and the difference $\Delta \chi^{2}=\chi^{2}-\chi_{\mathrm{mp}}^{2}$ is computed for each experiment. The critical value of the difference, $\Delta \chi_{\text {crit }}^{2}$, is defined as the one for which $\alpha$ fraction of the experiments at that combination of oscillation parameters have $\Delta \chi^{2}<\Delta \chi_{\text {crit }}^{2}$. Once the data are known, the difference between $\chi^{2}$ for each set of oscillation parameters and the best fit $\chi^{2}$ is computed. The points for which $\Delta \chi^{2}$ of data are is than $\Delta \chi_{\text {crit }}^{2}$ are included in the confidence interval with coverage $\alpha$. 
The Feldman-Cousins critical values of $\chi^{2}$ are shows in figure 13.12. The contours derived using these critical $\chi^{2}$ values are shown in figure 13.13. Note that this method does not change the best fit value, it only changes the region of phase space included in the confidence interval. The FC contours are noticeably less smooth than those in figure 13.10 that were based on Gaussian assumption. This is due to the limited number of experiments (10 million) that was run to produce this result, rather than anything inherent to the FC method.

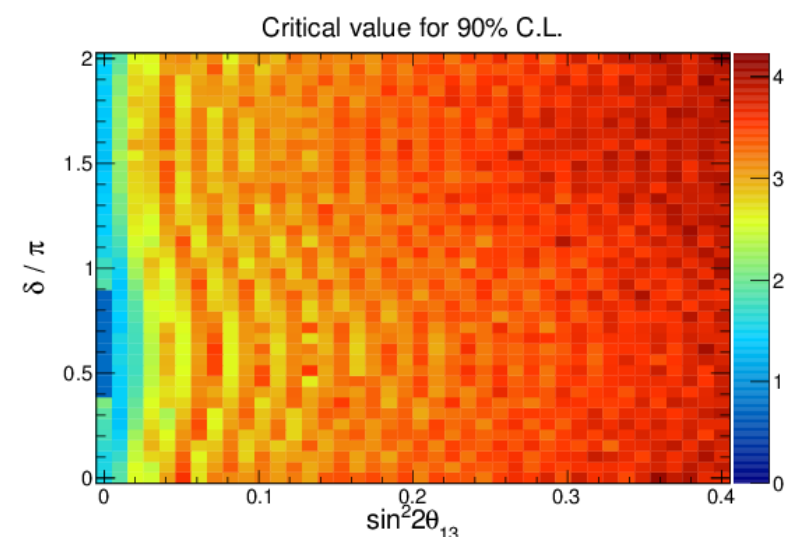

(a) Normal Hierarchy

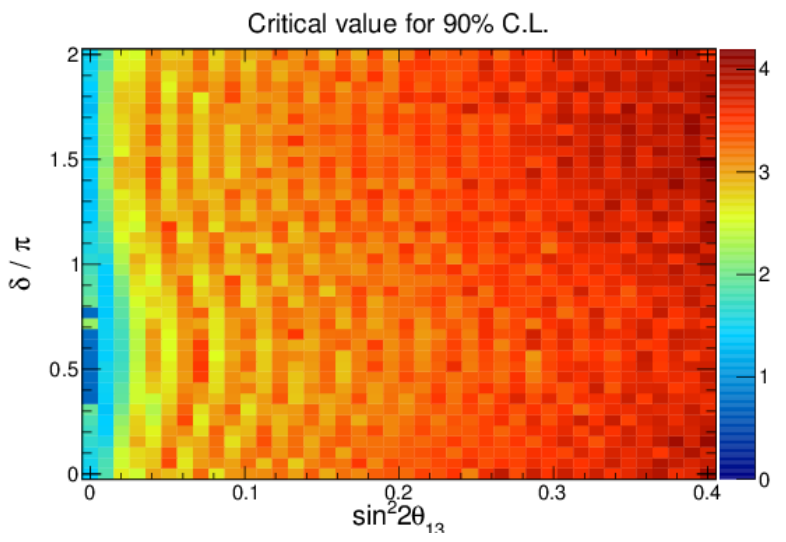

(b) Inverted Hierarchy

Figure 13.12: Critical value of $\chi^{2}$ in $\sin ^{2} 2 \theta_{13}$ vs $\delta$ space for normal and inverted hierarchies. 


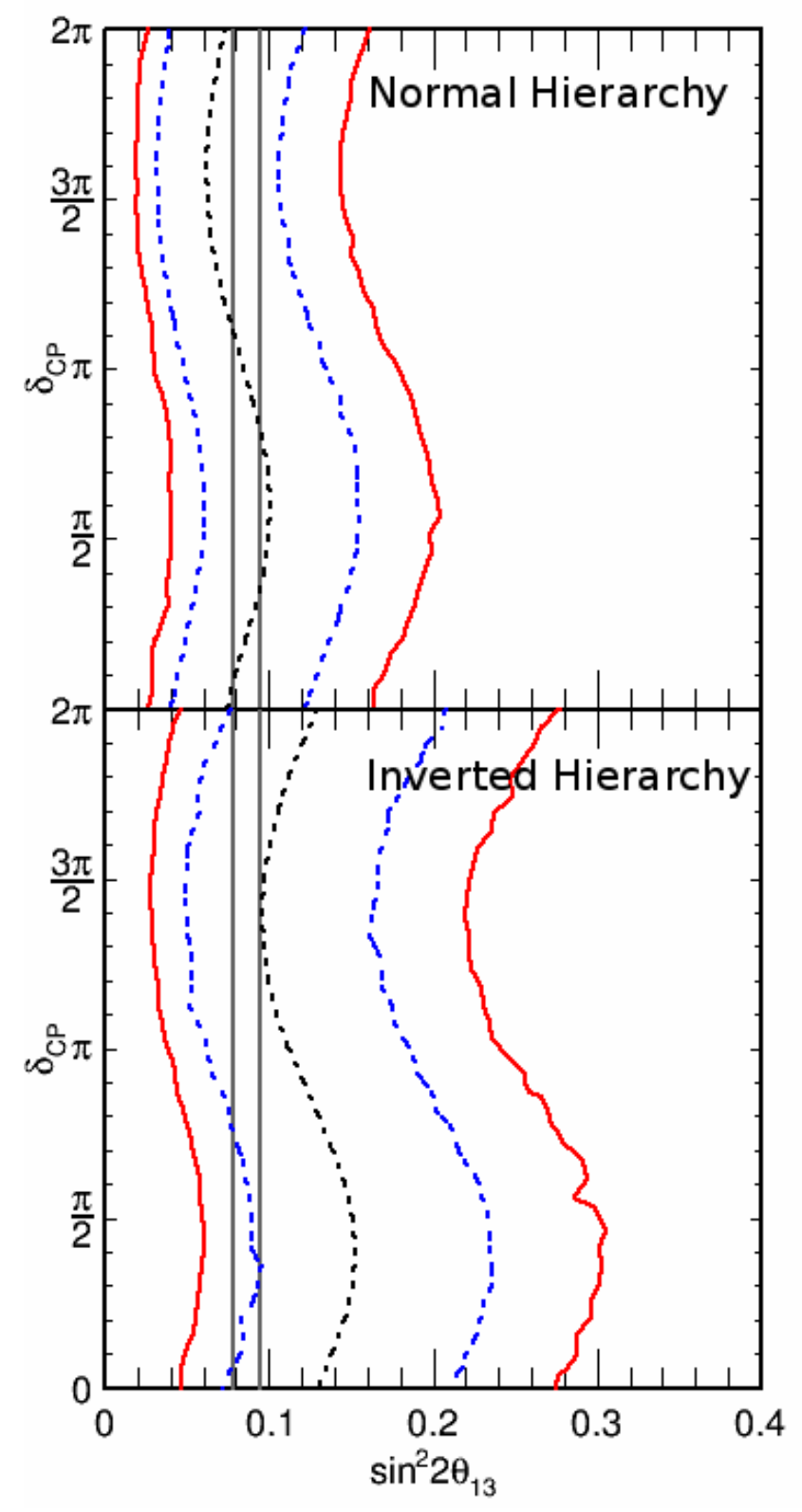

Figure 13.13: Feldman-Cousins contours for $68 \%$ and $90 \%$ confidence interval in $\theta_{13}$ and $\delta$ space, based on the observation of 6 events in the $\nu_{e}$ appearance channel, where the expected background is 0.97 event. The dotted blue lines enclose the $68 \%$ confidence interval and the solid red lines are $90 \%$ confidence interval. The solid gray lines show the $\sin ^{2} 2 \theta_{13}$ measurement from the reactor experiments. 


\subsubsection{Result from Alternative PID, LEM}

As mentioned before, LEM is an alternative PID developed for $\nu_{e}$ identification in $\mathrm{NO} \nu \mathrm{A}$. It matches every candidate event against a library of simulated neutrino interactions and deduces its identity based on the characteristics of the best matched simulated events in the library. For completeness, the LEM PID selects 11 events in the $\mathrm{NO} \nu \mathrm{A}$ first data. All 6 events selected by LID are also selected by LEM. Of the 5 additional events that LEM selects, 3 are in the LID sideband from 0.7 to 0.95. Given the estimated correlation $(62 \%)$ between the two PIDs, the probability of observing 5 events in LEM that are not selected by LID, and no events in LID that are not selected by LEM, and other less likely combinations is $9.2 \%$.

\subsubsection{Comparison with Official $\mathrm{NO} \nu \mathrm{A} \nu_{e}$ Appearance Result}

The officially presented and published $\nu_{e}$ appearance result from the $\mathrm{NO} \nu \mathrm{A}$ collaboration do not apply the MRE correction to the far detector signal estimate. The $\sim 12 \%$ systematic error in the official analysis on the $\nu_{e}$ signal estimate is meant to cover the MRE correction. Since the expected signal count in far detector is $\sim 15 \%$ lower than the MRE corrected signal count, this changes the interpretation of the observed 6 candidate events. Without the MRE correction, for normal hierarchy and $\mathrm{CP}$ violating phase, $\delta=3 \pi / 2$, the expected total event count (signal + background) is 5.62 events, which is consistent with the observation of 6 events, so this scenario is favored in the official result.

\subsection{Future Analyses of NO $\nu$ A Data}

The analysis presented here uses roughly $8 \%$ of the exposure planned for the full age of the $\mathrm{NO} \nu \mathrm{A}$ experiment. While higher statistics will play a large role in improving the power of future $\mathrm{NO} \nu \mathrm{A} \nu_{e}$ appearance results, many aspects of the analysis will need substantial improvement to keep systematic errors relatively low. The hadronic 
energy discrepancy that has been discussed at some length in this thesis is one of the highest priority issues to resolve over the next few months. There is evidence that the disagreement arises from under-representing quasi-elastic-like interactions in the simulation. A simple fit for scale factors of interaction types has been presented here; however, since the cross-sections of different modes are a function neutrino energy, an energy based fit might provide additional power. Applying external constraints, such as the neutrino cross-section measurements by the MINER $\nu \mathrm{A}$ experiment might also aid in resolving this discrepancy.

Cosmic background rejection in the far detector costs a considerable loss in efficiency of $\nu_{e}$ signal selection. For this analysis, a simple cut-based approach was used for cosmic rejection as proof of concept. The cuts were optimized to maximize the significance of $\nu_{e}$ appearance. For future analyses, multi-variate techniques can be explored for cosmic rejection to improve efficiency. Minimizing systematic error while tuning cuts such as the neutrino energy cut might also help to make the measurements more robust.

While many avenues of improvement remain open for exploration, the detectors are already performing remarkably well. The level of detail with which neutrino interactions can be observed is unparalleled for a detector of this size. As the NuMI beam continues to ramp up in power, the $\mathrm{NO} \nu \mathrm{A}$ data are making the remaining unmeasured neutrino oscillation parameters more accessible. T2K has recently presented their first data in the anti-neutrino mode, although it is too early to definitively claim $\bar{\nu}_{e}$ appearance [49]. Over the next year, plans to run the NuMI beam in anti-neutrino mode will be finalized as well. The following years promise to be very interesting in the field of neutrino physics. 


\section{References}

[1] Davide D'Angelo. Low Energy Neutrino Measurements. Pramana, 79:757-780, 2012, 1211.5359 .

[2] Robert M. Zwaska. Accelerator System and Instrumentation for the NuMI Neutrino Beam. PhD thesis, University of Texas, Austin, 2005.

[3] S. Mufson, B. Baugh, C. Bower, T. Coan, J. Cooper, et al. Liquid scintillator production for the NOvA experiment. 2015, 1504.04035.

[4] Fundamental Physics at the Intensity Frontier, 2012, 1205.2671.

[5] P. Hernandez. Neutrino physics. 2010, 1010.4131.

[6] K.A. Olive et al. Review of Particle Physics. Chin.Phys., C38:090001, 2014.

[7] Quang Ho-Kim and Pham Xuan Yem. Elementary Particles and Their Interactions. Springer, 1998.

[8] Don L Anderson. Theory of the Earth. Blackwell Scientific Publications , Boston, MA, 1989.

[9] J. N. Bahcall, W. A. Fowler, I. Iben, Jr., and R. L. Sears. Solar Neutrino Flux. The Astrophysical Journal, 137:344-346, January 1963.

[10] Raymond Davis, Don S. Harmer, and Kenneth C. Hoffman. Search for neutrinos from the sun. Phys. Rev. Lett., 20:1205-1209, May 1968. 
[11] John N. Bahcall, M. H. Pinsonneault, Sarbani Basu, and J. Christensen-Dalsgaard. Are standard solar models reliable? Phys. Rev. Lett., 78:171-174, Jan 1997.

[12] John N. Bahcall and Jr. Raymond Davis. The evolution of neutrino astronomy. Publications of the Astronomical Society of the Pacific, 112(770):pp. 429-433, 2000.

[13] F. Kaether, W. Hampel, G. Heusser, J. Kiko, and T. Kirsten. Reanalysis of the gallex solar neutrino flux and source experiments. Phys. Lett., B685:47-54, 2010, 1001.2731 .

[14] M. Altmann et al. Complete results for five years of gno solar neutrino observations. Phys. Lett., B616:174, 2005, hep-ex/0504037.

[15] J. N. Abdurashitov et al. Measurement of the solar neutrino capture rate with gallium metal, part iii. Phys. Rev., C80:015807, 2009, 0901.2200.

[16] B. Ananthanarayan and Ritesh K. Singh. Direct observation of neutrino oscillations at the sudbury neutrino observatory. Curr. Sci., 83:553, 2002, physics/0208096.

[17] Y. Fukuda et al. Evidence for oscillation of atmospheric neutrinos. Phys. Rev. Lett., 81:1562-1567, 1998, hep-ex/9807003.

[18] M. Apollonio et al. Search for neutrino oscillations on a long base-line at the chooz nuclear power station. Eur. Phys. J., C27:331, 2003, hep-ex/0301017.

[19] Y. Abe et al. Reactor electron antineutrino disappearance in the double chooz experiment. Phys. Rev., D86:052008, 2012, 1207.6632.

[20] F.P. An et al. Observation of electron-antineutrino disappearance at daya bay. Preprint, 2012.

[21] Kim Soo-Bong et al. Observation of reactor electron antineutrino disappearance in the reno experiment. 2012. 
[22] S. M. Bilenky and Carlo Giunti. Neutrinoless double-beta decay: A brief review. Mod. Phys. Lett., A27:1230015, 2012, 1203.5250.

[23] A. Cervera, A. Donini, M.B. Gavela, J.J. Gomez Cdenas, P. Hernndez, O. Mena, and S. Rigolin. Golden measurements at a neutrino factory. Nuclear Physics B, $579(12): 17-55,2000$.

[24] P. Adamson et al. Combined analysis of $\nu_{\mu}$ disappearance and $\nu_{\mu} \rightarrow \nu_{e}$ appearance in minos using accelerator and atmospheric neutrinos. Phys.Rev.Lett., 112:191801, 2014, 1403.0867.

[25] D.S. Ayres et al. The nova technical design report. Technical report, Fermilab, Batavia, Illinois, 2007.

[26] E. Niner, P. Adamson, G. Deuerling, R. Kwarciany, H. Meyer, et al. Synchronization of the $14 \mathrm{kTon} \mathrm{NO} \nu \mathrm{A}$ neutrino detector with the Fermilab NuMI beam. J.Phys.Conf.Ser., 513:012028, 2014.

[27] Alexander Radovic. Measuring the Disappearance of Muon Neutrinos with the MINOS Detector. PhD thesis.

[28] C. Andreopoulos et al. The GENIE Neutrino Monte Carlo Generator. Nucl. Instrum. Meth., A614:87-104, 2010, 0905.2517.

[29] Chris Hagmann et al. Cosmic-ray Shower Library (CRY).

[30] S. Agostinelli et al. GEANT4: A Simulation toolkit. Nucl.Instrum.Meth., A506:250-303, 2003.

[31] A Aurisano et al. The nova simulation chain. Submitted to Journal of Physics: Conference Series.

[32] Jonathan Liu. Quantifying croscross in avalanche photodiodes used in nova. NOvA internal document database. 
[33] J.B. Birks. The theory and practice of scintillation counting. International series of monographs on electronics and instrumentation. Macmillan, 1964.

[34] C. N. Chou. The nature of the saturation effect of fluorescent scintillators. Phys. Rev., 87:904-905, Sep 1952.

[35] Martin Ester, Hans peter Kriegel, Jrg S, and Xiaowei Xu. A density-based algorithm for discovering clusters in large spatial databases with noise. pages 226-231. AAAI Press, 1996.

[36] L. Fernandes and M. Oliveira. Real-timelinedetectionthroughanimprovedhoughtransform votingscheme. Pattern Recognition, 41:299-314, 2008.

[37] Mattias Ohlsson. Extensions and explorations of the elastic arms algorithm. Computer Physics Communications, 77(1):19 - 32, 1993.

[38] J. C. Dunn. A fuzzy relative of the isodata process and its use in detecting compact well-separated clusters. Journal of Cybernetics, 3(3):32-57, 1973, http://dx.doi.org/10.1080/01969727308546046.

[39] R. Krishnapuram and J.M. Keller. A possibilistic approach to clustering. Fuzzy Systems, IEEE Transactions on, 1(2):98-110, May 1993.

[40] Miin-Shen Yang and Kuo-Lung Wu. Unsupervised possibilistic clustering. Pattern Recogn., 39(1):5-21, January 2006.

[41] Rudolph Emil Kalman. A new approach to linear filtering and prediction problems. Transactions of the ASME-Journal of Basic Engineering, 82(Series D):35-45, 1960.

[42] C. Backhouse and R. B. Patterson. Library event matching event classification algorithm for electron neutrino interactions in the nova detectors. Nucl.Instrum.Meth., A778:31-39, 2015, 1501.00968. 
[43] Gavin Davies et al. Position paper: Nue systematics. NOvA Internal Document Database 13597 http://nova-docdb.fnal.gov:8080/cgibin/ShowDocument?docid=13597.

[44] V.I. Tretyak. Semi-empirical calculation of quenching factors for ions in scintillators. Astroparticle Physics, 33(1):40 - 53, 2010.

[45] Hongyue Duyang et al. A technote describing the derivation and size of numi flux uncertainties used in the first nova analyse. NOvA internal Document Database 13584, http://nova-docdb.fnal.gov:8080/cgi-bin/ShowDocument?docid=13584.

[46] Nicholas Raddatz. Fiducial mass systematic. NOvA Internal Document Database 13237 http://nova-docdb.fnal.gov:8080/cgi-bin/ShowDocument?docid=13237.

[47] K. Abe et al. Measurements of neutrino oscillation in appearance and disappearance channels by the T2K experiment with $6.610^{20}$ protons on target. Phys. Rev., D91(7):072010, 2015, 1502.01550.

[48] Gary J. Feldman and Robert D. Cousins. Unified approach to the classical statistical analysis of small signals. Phys. Rev. D, 57:3873-3889, Apr 1998.

[49] Melody RAVONEL. Antineutrino oscillations with t2k. 2015. EPS HEP 2015. 